\title{
A phosphoinositide conversion mechanism for exit from endosomes
}

\author{
Inaugural-Dissertation
}

to obtain the academic degree

Doctor rerum naturalium (Dr. rer. nat.)

submitted to the Department of Biology, Chemistry and Pharmacy

of Freie Universität Berlin

by

KATHARINA KETEL

from Berlin, Germany

2016 
Period of doctorate study:

Supervisor:

Institute:

Prof. Dr. Christian Freund
January 2011 until February 2016

Prof. Dr. Volker Haucke

January 2011 until August 2012: Institute of Chemistry and Biochemistry, Freie Universität Berlin

September 2012 until February 2016: Leibniz Institute for Molecular Pharmacology (FMP), Berlin $1^{\text {st }}$ Reviewer:

$2^{\text {nd }}$ Reviewer:

Prof. Dr. Volker Haucke

Date of defense: June $6^{\text {th }} 2016$ 


\section{Acknowledgements}

I am deeply grateful for the excellent training and guidance I received from my supervisor Prof. Volker Haucke during the time of my $\mathrm{PhD}$ thesis as well as throughout my undergraduate studies. Your willingness to extensively and very frequently discuss every little detail of my project, your ability to guide me through difficult parts of the project and your enthusiasm for science were a constant source of motivation and essential for the success of my project.

I owe special thanks to Marnix Wieffer and Michael Krauß, who spent a lot of time helping me to get started in the lab, to overcome numerous experimental problem, I was not sure how to deal with, and frequently joined in discussions about my project with excellent advice. I am really grateful for your experimental support, Michael, and the time you invested in the project during our paper revision.

Further, I would like to thank Jocelyn Laporte and his group, especially Anne-Sophie Nicot, at the IGBMC in Strasbourg for their support and a very fruitful collaboration, and Carsten Schultz and his group at the EMBL in Heidelberg for their support and constant supply with PIP/AMs. I owe special thanks to Dmytro Puchkov for the ultrastructural analysis and Eberhard Krause for mass spectrometry done within this project. I also need to acknowledge Markus Wenk and Federico Torta at the Singapore Lipidomics Incubator for joining the project at a very late stage, when we urgently needed help from lipidomics specialists. It was a pity that experiments did not work out as planned.

I would further like to express my gratitude to two very skilled technicians in the AG Haucke lab: Silke Zillmann and Delia Löwe. Without your help it would have been impossible to achieve so much within the limited amount of time I had during the paper revision.

Further, I would like to acknowledge all past and present members of the AG Haucke lab, including the AG Schmoranzer, AG Maritzen and AG Krauß. Without you work would have been less cheerful, enjoyable and efficient: Jelena Bacetic, Marietta Browarski, Caraoline Bruns, Katrin Diesenberg, Marielle Eichborn-Grüning, Fabian Feutlinske, Uwe Fink, Niclas Gimber, Gala Claßen, Claudia Gras, Isabelle Grass, Kira Gromova, Sabine Hahn, Burkhard Jakob, Lisa Jerndal, Maria Jäpel, Natalie Kaempf, Christina Kath, Peter Koch, 
Gaga Kochlamazashvili, Natalia Kononenko, Seong Joo Koo, Ludwig Krabben, Marijn Kuijpers, André Lampe, Wen-Ting Lo, Martin Lehmann, Gregor Lichtner, Tania López Hernández, Marta Maglione, Andrea Marat, Tanja Maritzen, Julia Mössinger, Maria Mühlbauer, Arndt Pechstein, Jasmin Podufall, York Posor, Yijian Rao, Christine Rückert, Linda Sawade, Hannah Schachtner, Claudia Schmidt, Jan Schmoranzer, Irene Schütz, Kyungyeun Song, Wiebke Stahlschmidt, Tolga Soykan, Susanne Thomsen, Anela Vukoja, Alexander Wallroth, Ingeborg Walther, Susanne Wojtke, Haibin Wang, Anna Wawrzyniak and Mirjana Weimershaus.

Most importantly, I would like to express my gratitude to my parents, my husband and my brother who supported me throughout my thesis, no matter how time consuming, and made everyday life beside work doable. The knowledge that I have your support no matter what I decide to do is most reassuring. 


\section{Table of Content}

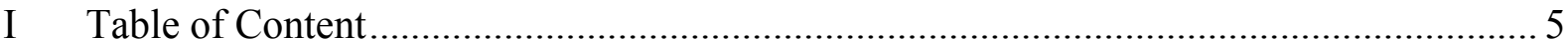

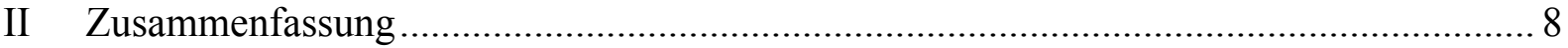

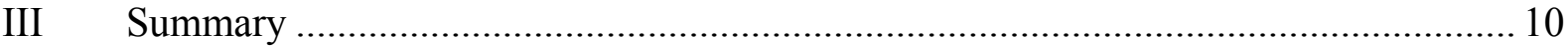

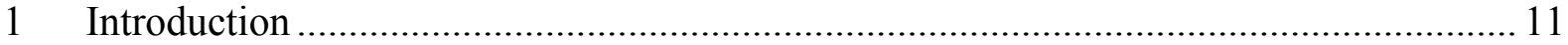

1.1 Defective PI homeostasis and membrane remodeling might cause X-linked

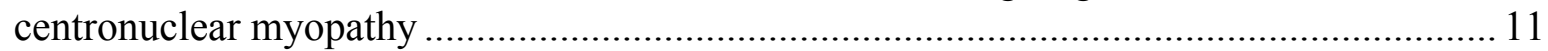

1.1.1 Exocytic membrane transport is counterbalanced by retrograde traffic ..........11

1.1.2 Phosphoinositides determine membrane identity ............................................ 12

1.2 Membrane traffic is regulated by phosphoinositides ............................................ 15

1.2.1 Phosphoinositides serve as spatio-temporal landmarks .................................. 15

1.2.2 PI(4,5) $P_{2}$-to-PI(3)P conversion in endocytic membrane traffic ...................... 16

1.2.3 Endosomal sorting occurs via tubular cargo-enriched subdomains ................ 17

1.2.4 Exocyst tethering in endosomal exocytosis is regulated by endosomal and cell surface determinants .................................................................................... 20

1.3 Myotubularins are a family of PI 3-phosphatases ..................................................2 22

1.3.1 MTM1 regulates endosomal PI(3)P turnover and membrane dynamics .........23

1.3.2 Other myotubularin family members contribute to endosomal PI(3)P turnover

1.4 Endosomal PI(4)P is synthesized by type II PI 4-kinases ...................................2. 26

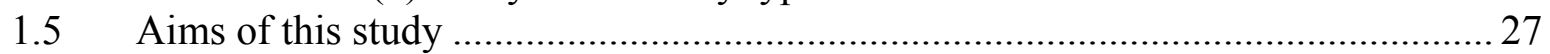

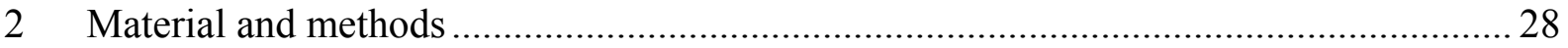

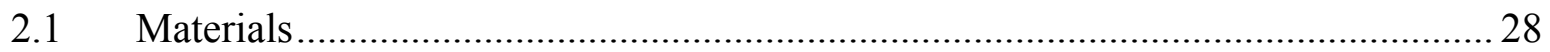

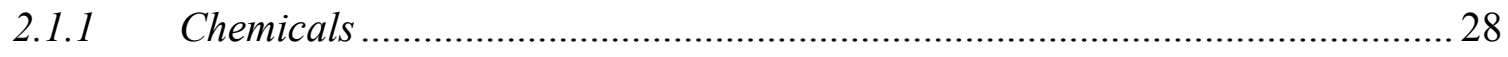

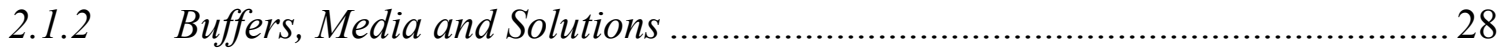

2.1.3 DNA Oligonucleotides............................................................................. 31

2.1.4 Small interference RNA Oligos ................................................................... 31

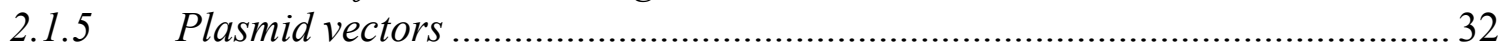

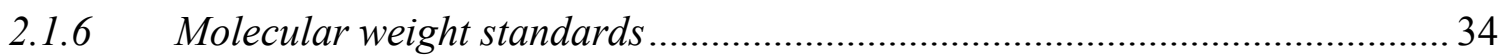

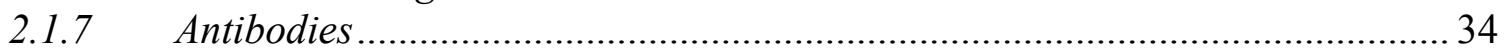

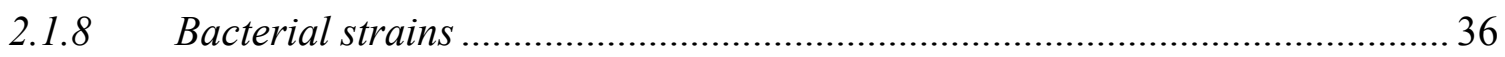

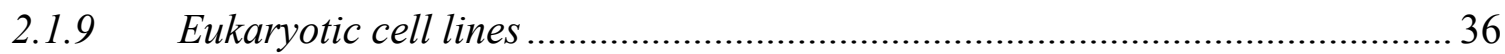

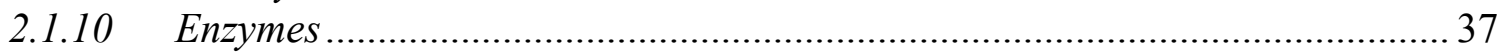

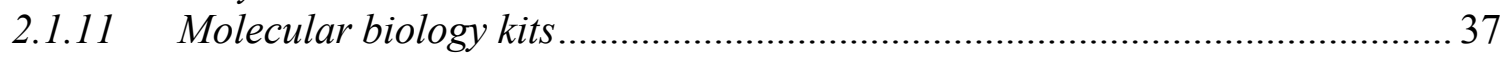

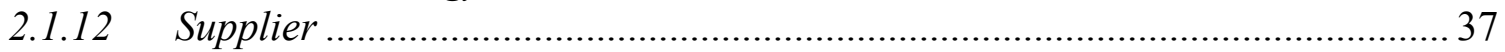

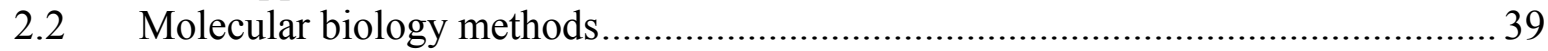

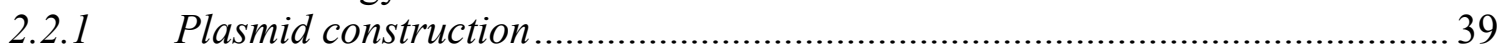

2.2.2 Preparation of complementary DNA (cDNA) libraries .....................................39

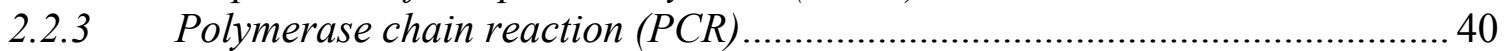

2.2.4 Analytical agarose gel electrophoresis ......................................................... 42

2.2.5 Purification of DNA from agarose gels and PCR ……................................ 42

2.2.6 Restriction digest ...................................................................................... 42

2.2.7 Integration of $P C R$ products into linearized vector backbones ........................ 43

2.2.8 Preparation of chemically competent E. coli .................................................. 43

2.2.9 Transformation of chemically competent E.coli ............................................. 44

2.2.10 Isolation of DNA from bacterial cultures ......................................................... 44

2.2.11 Measurement of DNA concentrations ............................................................. 44

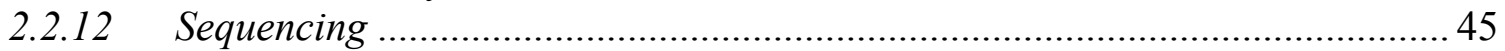

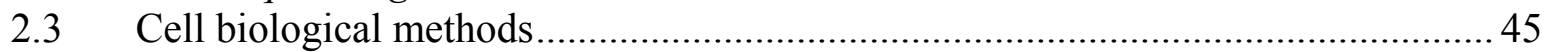

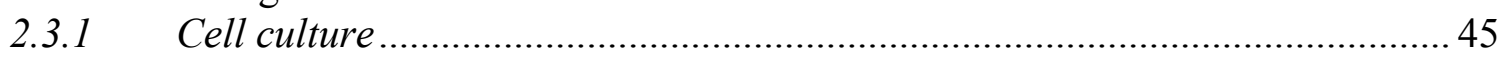




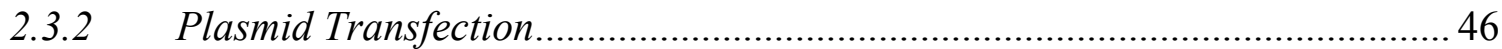

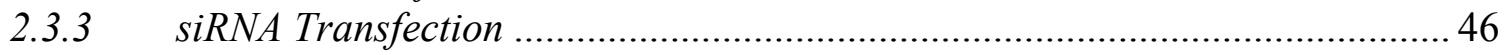

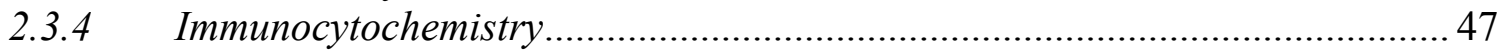

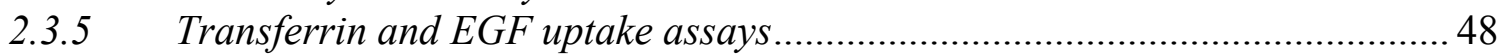

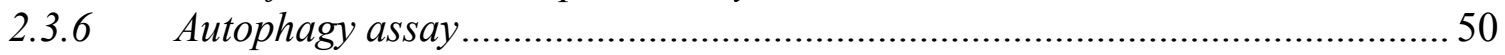

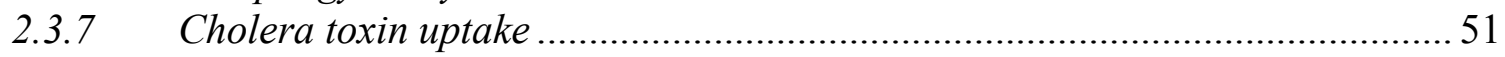

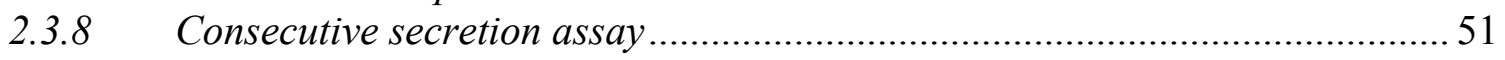

2.3.9 Elevation of PI(3)P and PI(4)P by PIP/AM treatment .................................. 51

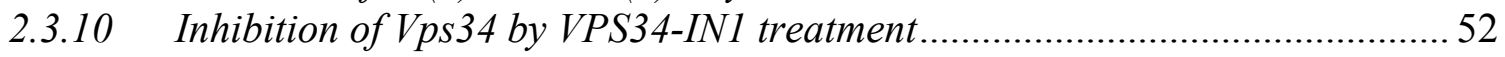

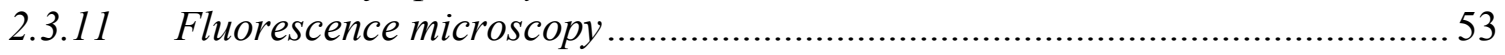

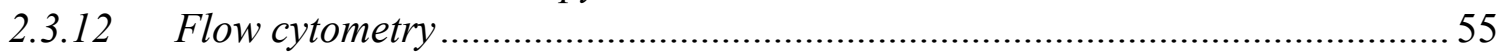

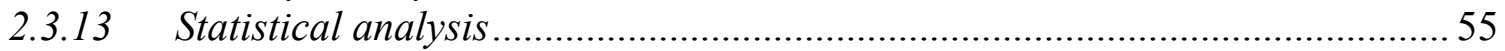

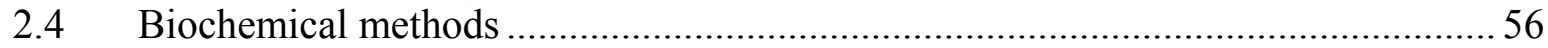

2.4.1 Expression of recombinant proteins in E. coli ................................................ 56

2.4.2 Purification of His ${ }_{6}$-tagged MTM1 fragment expressed in E. coli for

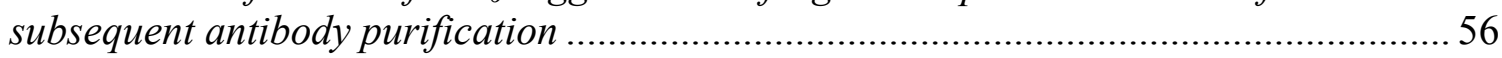

2.4.3 Affinity purification of polyclonal MTMlantibody from serum ........................ 57

2.4.4 Preparation of cell extracts from mammalian cell cultures.............................. 58

2.4.5 Determination of protein concentration using Bradford assay ........................ 59

2.4.6 Sodiumdodecylsulfate polyacrylamide gel electrophoresis (SDS-PAGE) ....... 59

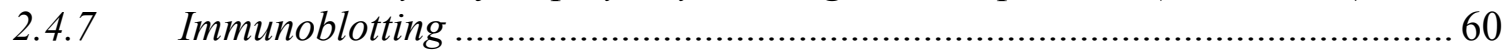

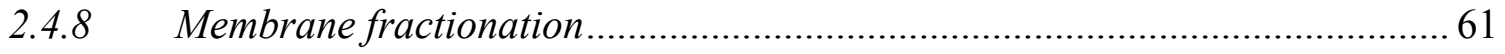

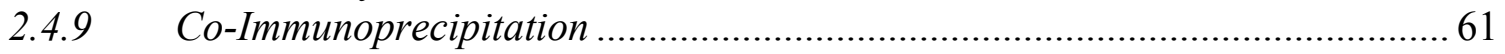

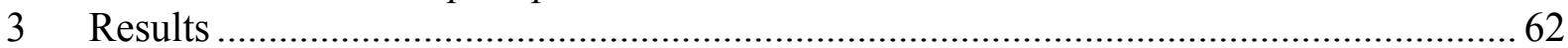

3.1 Exocytosis from endosomes is impaired in XLCNM patient cells .......................... 62

3.1.1 $\quad$ 1-integrin accumulates in late and recycling endosomes upon loss of MTM1

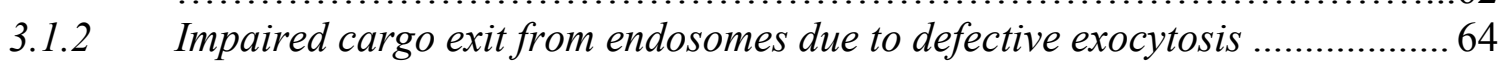

3.2 Tf exocytosis from endosomes requires MTM1-mediated PI(3)P hydrolysis ......... 66

3.2.1 The subcellular distribution of TfR is altered upon loss of MTM1 ...................68

3.2.2 Endosomal trafficking and cargo sorting in MTM1-depleted cells ................. 71

3.2.3 Other Myotubularin family members in TfR exocytosis .................................. 73

3.3 Endosomal accumulation of PI(3)P and PI(3)P effector proteins inhibits exocytosis

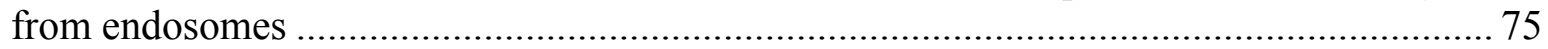

3.3.1 Sub-plasma membrane early and recycling endosomes accumulate upon loss of

MTM1 ….............................................................. 75

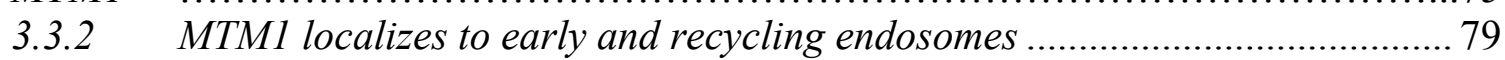

3.3.3 PI(3)P accumulates on endosomes in MTM1-depleted cells ............................. 80

3.3.4 PI(3)P levels can be manipulated by genetic and pharmacological tools ....... 83

3.4 PI(3)P-to-PI(4)P conversion is required for exocyst-dependent endosomal

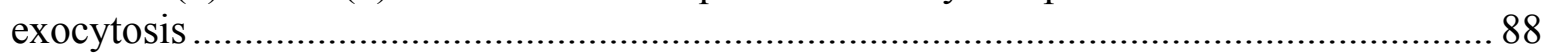

3.4.1 Exocytic vesicles acquire PI(4)P before plasma membrane fusion ................. 88

3.4.2 PI4K2 $\alpha$ and exocyst associate and are required for exocytosis ...................... 90

3.4.3 Rab11 is required for endosomal exocyst localization ....................................93

3.4.4 Exocyst recruitment to endosomes depends on PI4K2 $\alpha$ and PI(4)P............... 95

3.4.5 PI4K2 $\alpha$-mediated MTM1 recruitment to endosomes initiates PI(3)P-

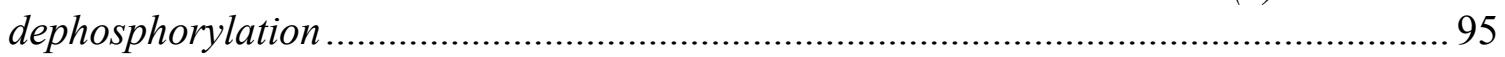

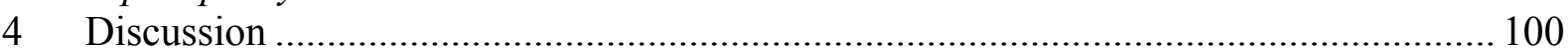

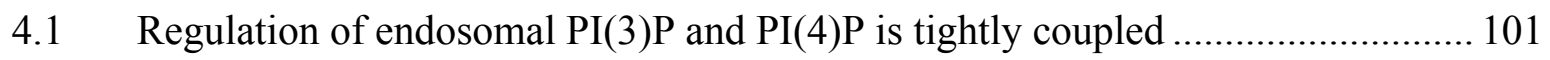

4.1.1 Endosomal PI(3)P levels are balanced by 3'-metabolizing enzymes ............ 101

4.1.2 Membrane recruitment of MTM1 is regulated by lipid and protein content 103 
4.1.3 PI(3)P and PI(3,5)P $P_{2}$ are lipid substrates of MTM1 104

4.1.4 PI4K2 $\alpha$-dependent PI(4)P synthesis defines endosomal subdomains for cargo sorting

4.1.5 PI(3)P turnover regulates endosomal tubulation and cargo sorting..... 105

4.2 PI(3)P-to-PI(4)P conversion regulates endosomal recycling ................................. 109

4.2.1 PI conversion in endosomal exocytosis parallels switching of the Rab identity

4.2.2 Regulation of the exocyst complex by endosomal and PM determinants

4.3 Common features of MTM1 deficiency cause XLCNM pathologies.....

4.3.1 Late endosomal recycling contributes to $\beta 1$-integrin traffic.

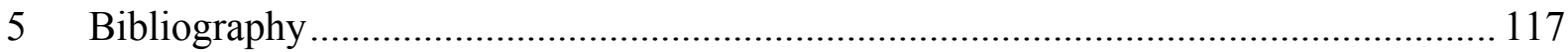

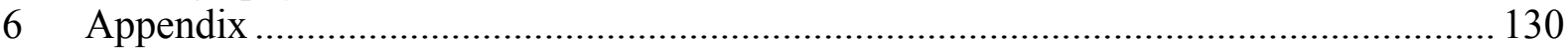

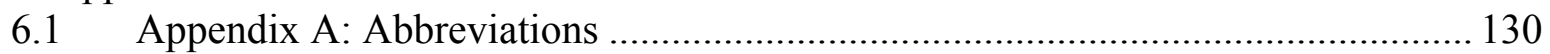

6.2 Appendix B: Mass spectrometry results............................................................... 133

6.2.1 MTM1 binding partners identified by mass spectrometry ............................. 133

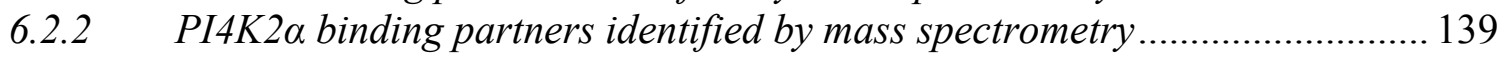

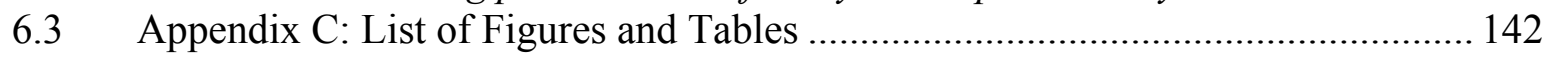

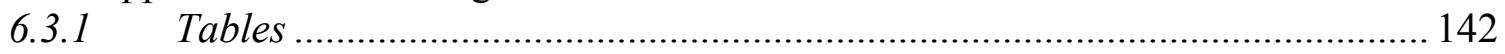

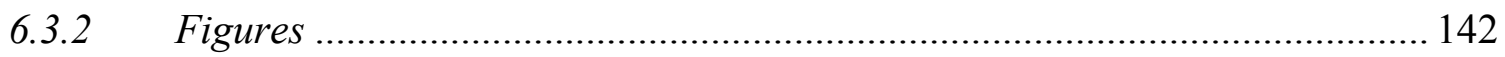

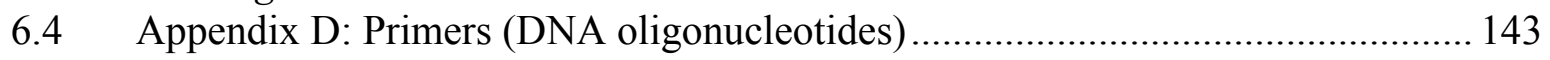

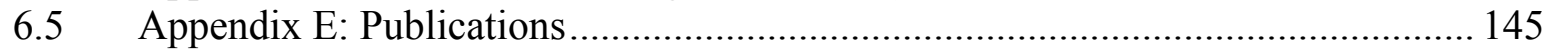




\section{Zusammenfassung}

Phosphoinositide (PI) sind eine seltene Gruppe von kurzlebigen Phospholipiden, die als Organisationsprinzip von Membranen hervorgetreten sind und Zellmembranen ihre Identität verleihen. Hierdurch erfüllen sie essentielle Funktionen in der Signaltransduktion und dem Membrantransport. PI 4-Phosphate wie Phosphatidylinositol-4-Phosphat (PI(4)P) und Phosphatidylinositol-4,5-Bisphosphat $\left(\mathrm{PI}(4,5) \mathrm{P}_{2}\right)$ sind an der Plasmamembran (PM), auf sekretorischen Organellen und Lysosomen angereichert, während PI 3-Phosphate, z.B. Phosphatidylinositol-3-Phosphat (PI(3)P), das Endomembransystem kennzeichnen. Gerichteter Transport zwischen diesen Kompartimenten benötigt daher die kontrollierte Konversion von PIs. Dennoch ist die PI Konversion auf dem Weg von PI(3)P-reichen Endosomen hin zur PI(4)P- und PI(4,5) $\mathrm{P}_{2}$-angereicherten $\mathrm{PM}$ in endosomalem Recycling nur unzureichend verstanden.

In dieser Studie legen wir dar, dass der Austritt von Cargoproteinen aus Endosomen die Entfernung von PI(3)P durch die PI(3)P 3-Phosphatase Myotubularin 1 (MTM1) benötigt sowie die anschließende Synthese von PI(4)P durch PI 4-Kinase Typ II $\alpha$ (PI4K2 $\alpha$ ). Der Verlust von MTM1 führt zur endosomalen Akkumulation von PI(3)P und PI(3)P-Effektoren, zum Kif16b-vermittelten auswärtsgerichteten Transport von PI(3)P-reichen Endosomen und der Akkumulation von Exozytose-defizienten Endosomen unterhalb der PM. Da PI4K2 $\alpha$ mit MTM1 assoziiert und hierdurch die Rekrutierung von MTM1 auf endosomale Membranen ermöglicht, spiegelt der Verlust von PI4K2 $\alpha$ diese phenotypischen Veränderungen wider. Die Konversion von PI(3)P zu PI(4)P wird begleited durch einen Wechsel in der Identität der Rab GTPasen. Hierbei wird der frühe endosomale Marker Rab5 durch den Marker für RecyclingEndosomen, Rab11, ersetzt. Dieser Konversionsmechanismus wird angetrieben durch die Koinzidenz-Detektion von Rab GTPasen und PIs sowie durch reziproke Interaktionen zwischen den Komponenten. Diese beinhalten die Assoziation des Exozyst-Komplexes mit PI4K2 $\alpha$ und PI(4)P, Rab11 und mit MTM1. Hierdurch werden endosomale Subdomänen für endosomales Recycling geschaffen, die durch PI4K2 $\alpha$, dessen Lipidprodukt PI(4)P, Rab11 und MTM1 gekennzeichnet sind. Auf diesen Subdomänen kann der Exozyst-Komplex assembliert werden, in dessen Abhängigkeit exozytische Vesikel mit der PM fusionieren. Ein ähnlicher PI Konversionsmechanismus, der Myotubularine beinhaltet, könnte den Austritt von Cargoproteinen aus frühen Endosomen hin zu späten Endosomen kontrollieren. Wesentlich hierbei ist, dass der Verlust von MTM1 und XLCNM-assoziierte Mutationen funktionell stark 
korrelieren. Fibroblasten von XLCNM-Patienten zeigen Exozytosedefekte und akkumulieren $\beta 1$-Integrin in Endosomen. Unsere Daten legen daher nahe, dass defekte PI Konversion auf Endosomen für die gestörte Muskelmorphologie und Defizite in der Integrin-basierten Muskeladhäsion in sich entwickelnden Muskeln von XLCNM Patienten verantwortlich ist. Diese Misslokalisation von Integrinen kann in Zellkultur durch die Behandlung mit einem Vps34-spezifischen Inhibitor aufgehoben werden und stellt dadurch eine Möglichkeit dar, dieser unheilbaren Krankheit entgegen zu wirken. 


\section{Summary}

Phosphoinositides (PIs) are a minor class of short-lived phospholipids that serve as crucial signposts of membrane identity. Thereby, PIs full fill important functions in cell signaling and membrane transport. PI 4-phosphates such as phosphatitylinositol-4-phosphate (PI(4)P) and phosphatitylinositol-4,5-bisphosphate $\left(\mathrm{PI}(4,5) \mathrm{P}_{2}\right)$ are enriched at the plasma membrane (PM), on secretory organelles and lysosomes, while PI 3-phosphates, i.e. phosphatitylinositol-3-phosphate (PI(3)P), are a hallmark of the endosomal system. Directional transport between these compartments, thus, requires regulated PI conversion. However, PI conversion in exit from PI(3)P-enriched endosomes en route to the PI(4)P- and $\mathrm{PI}(4,5) \mathrm{P}_{2}$-positive $\mathrm{PM}$ in endosomal recycling remained unknown.

Here, we report that cargo exit from endosomes requires removal of PI(3)P by the PI(3)P 3-phosphatase myotubularin 1 (MTM1), and concomitant PI(4)P synthesis by PI 4kinase type II $\alpha$ (PI4K2 $\alpha)$. Loss of MTM1 causes endosomal accumulation of PI(3)P and PI(3)P effector proteins, i.e. sorting nexins, Kif16b-mediated outward traffic of PI(3)P containing endosomes and sub-plasmalemmal accumulation of exocytosis-deficient endosomes. As PI4K2 $\alpha$ associates with MTM1 and thereby facilitates membrane recruitment of MTM1, these phenotypic changes are mimicked by loss of PI4K2 $\alpha$. The conversion of PI(3)P-to-PI(4)P is paralleled by a switch in Rab GTPase-identity from early endosomal Rab5 to recycling endosomal Rab11. This conversion mechanism is driven by coincident detection of Rab GTPase and PI content as well as by reciprocal interactions among its components, including the association of exocyst with PI4K2 $\alpha$ and PI(4)P, Rab11 and with MTM1. Thus, distinct recycling endosomal domains, characterized by PI4K2 $\alpha$, its lipid product PI(4)P, MTM1 and Rab11, are created destined for exocyst recruitment and subsequent exocystdependent fusion with the PM. A similar PI conversion mechanism involving myotubularin family members may underlie cargo exit from endosomes towards late endosomes. Importantly, MTM1 depletion and XLCNM-patient mutations are functionally correlated as patient fibroblast are exocytosis deficient and accumulate endosomal $\beta 1$-integrin. Our data, thus, suggest that defective PI conversion at endosomes may account for the disturbed muscle morphology and impaired integrin-dependent muscle attachment in developing muscle fibres in XLCNM-patients. These integrin localization defects can be reversed by application of a Vps34-specific inhibitor and thus offer a potential avenue to combat this devastating disease. 


\section{Introduction}

\subsection{Defective PI homeostasis and membrane remodeling might cause X-linked centronuclear myopathy}

X-linked centronuclear myopathy (XLCNM) is a form of congenital myopathy, characterised by generalized muscle weakness and hypotonia at birth. Pathologically, muscle fibers from XLCNM-patients are small and round with internally located myonuclei, surrounded by an area devoid of contractile elements and an accumulation of dysfunctional mitochondria. The gene mutated in XLCNM codes for the phosphatidylinositol-3-phosphate (PI(3)P) 3-phosphatase myotubularin 1 (MTM1). The most severe forms are associated with mutations resulting in MTM1 truncations or in loss of its catalytic activity. Resembling signs of XLCNM, MTM1 knockout (KO) mice are born below Mendelian ratio, display impaired growth and progressive myopathy leading to death within 2 to 3 months after birth. The conditional, muscle-specific MTM1 KO in mice did not impair muscle development, but structural maintenance of muscle architecture (Buj-Bello, Laugel et al. 2002). The latter requires constant remodeling of their plasma membrane content, regulated by intracellular and exocytic membrane traffic. Accordingly, muscle tissue from XLCNM patients or from MTM1-deficient mice displays endosomal accumulation of major cell adhesion proteins, e.g. integrins (Ribeiro, Yuan et al. 2011), and abnormal T-tubule morphology. Thus, defective PI(3)P turnover by loss of MTM1 might be linked to endosomal membrane traffic. Alternatively, defective autophagic and proteasomal degradation as well as upstream signaling events in MTM1-deficient mice were proposed to be the primary cause of the muscle pathology in XLCNM (Al-Qusairi, Prokic et al. 2013; Fetalvero, Yu et al. 2013), but will be discussed only briefly in the present study.

\subsubsection{Exocytic membrane transport is counterbalanced by retrograde traffic}

Anterograde membrane transport comprises secretory traffic from the trans-Golgi network (TGN) and exocytic membrane traffic from endosomes to the PM. The latter can be roughly divided into the fast and slow recycling mode from peripheral early and perinuclear recycling endosomes, respectively. To compensate for anterograde traffic to the cell surface, lipid and proteins are endocytosed from the plasma membrane, predominantly via clathrin- 
mediated endocytosis (CME). Homotypic fusion of endocytic vesicles with each other or with pre-existing endosomes generates the early endosomal compartment. Early endosomes can mature to late endosomes and form multivesicular bodies (MVB) en route to lysosomes for subsequent degradation. In addition, cargo can be transported back to the TGN, directly recycle to the PM or progress through the perinuclear recycling compartment en route to the PM (Figure 1). Thus, anterograde and retrograde transport is connected via a continuum of sorting stations, comprising early, late and recycling endosomes.

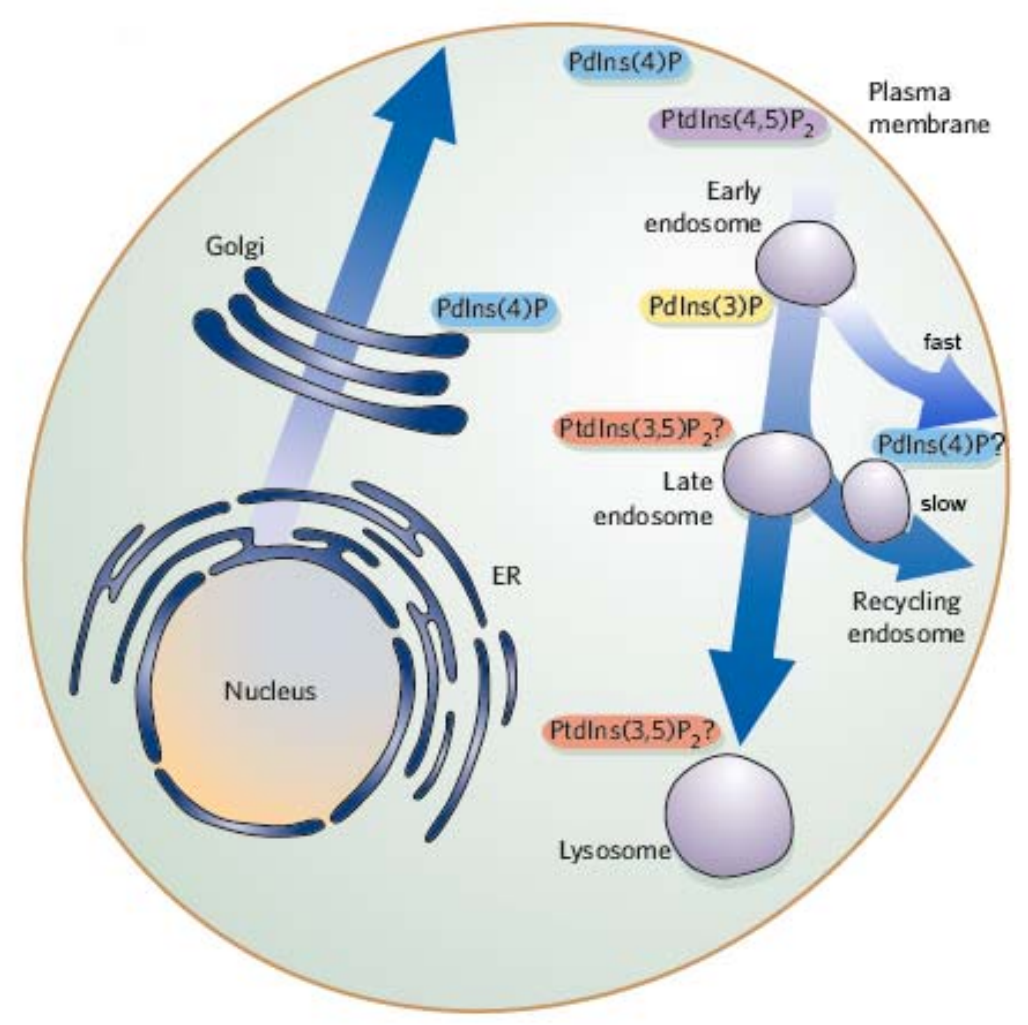

Figure 1: Distinct PI-pools as sign post of organelle identity in intracellular membrane traffic

PIs are converted as transport occurs along the secretory and endocytic routes. Notably, no PI species has been assigned to recycling endosomes so far. Modified from (Behnia and Munro 2005).

As membrane traffic occurs compartmental identity has to maintained and converted from the donor compartment characteristics to match the acceptor compartment. One crucial determinant is membrane lipid composition, most notably the predominant phosphoinositide (PI) species (Figure 1).

\subsubsection{Phosphoinositides determine membrane identity}

Organelle specific distribution of PI species is a key determinant of membrane identity, created by the compartment-specific subset of PI metabolizing enzymes. Thereby, a PI code is 
generated that can be recognized by PI effector proteins, ranging from proteins involved in membrane transport and remodeling to cytoskeleton organization, signaling and autophagy. However, PIs are rare lipids, comprising $5-10 \%$ of the total lipid pool whereas structural phospholipids i.e. phosphatidylserine (PS) are more abundant with $8.5 \%$ of total lipids (Wenk, Lucast et al. 2003; Lemmon 2008). This emphasizes the fundamental importance and regulatory role of PIs in processes such as membrane trafficking and signaling,
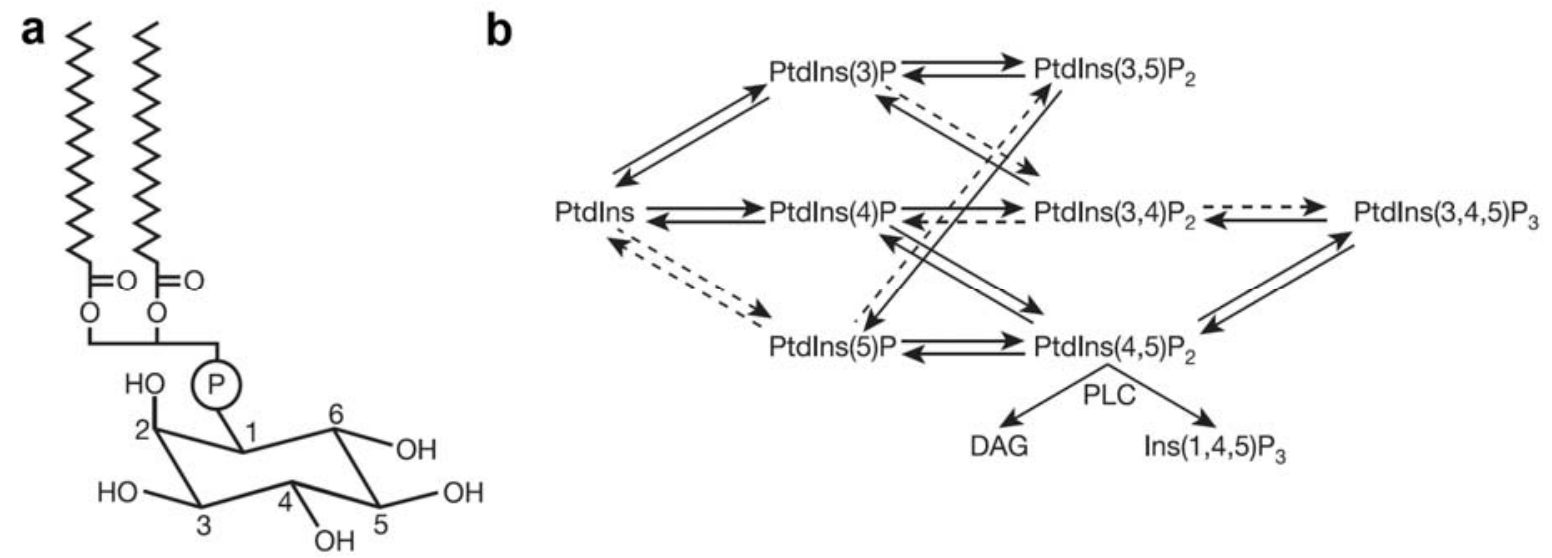

Figure 2: Phosphoinositide conversion

(a) Phosphatidylinositol is an amphiphatic membrane component that consists of phosphatidic acid and D-myoinositol as a hydrophilic headgroup. The glycerol backbone of phosphatidic acid is esterified at $\mathrm{C} 1$, predominantly to saturated fatty acid side chains, and at $\mathrm{C} 2$, predominantly to unsaturated fatty acid side chains. The non planar inositol ring structure contains hydroxyl groups at position 4' and 6' in trans configuration. (b) PIs can be reversibly phosphorylated at the $3^{\prime}, 4^{\prime}$ and $5^{\prime}$ position of the inositol ring. This interconversion is mediated by PI kinases and phosphatases. Finally, $\mathrm{PI}(4,5) \mathrm{P}_{2}$ can be cleaved by phospholipase C (PLC) into the integral membrane component DAG and cytosolic Inositol-1,4,5-trisphosphate $\left(\mathrm{I}(1,4,5) \mathrm{P}_{3}\right)$, both involved in Cadependent signaling cascades (Di Paolo and De Camilli 2006).

The group of PIs consists of phosphatidylinositol (PI) and its polyphosphorylated derivatives. PIs are amphipathic lipids, containing a hydrophobic diacylglycerol backbone that carries a phosphate headgroup esterified to the 1' positon of the myo-inositol ring (Figure 2a). The hydroxylgroups of the myo-inositol ring can be reversibly phosphorylated at the 3', 4' and 5' position by PI kinases and phosphatases, generating seven different PI species in total (Figure 2b) (De Matteis and Godi 2004; Di Paolo and De Camilli 2006). Further, the hydroxylgroups are stereochemically oriented in a "chair" conformation with the hydroxylgroup at position 2' being axial and all other positions being equatorial to the ring. Thus, stereospecific orientation of the phosphate headgroups, together with changes in general membrane properties such as curvature, charge or amphiphilicity ensures specific recognition by effector proteins. Herein, curvature can be generated by lipid shapes, classified as cylindric, cone-shaped or inverted cone-shaped depending on the size ratio between the 
headgroup and the fatty acid side chains, or by membrane-binding, curvature generating protein, i.e. BAR (Bin/ Amphiphysin/ Rys) - domain containing sorting nexins (SNX) (van Weering, Verkade et al. 2010).

PI is synthesized primarily on the cytoplasmic leaflet of the endoplasmic reticulum (ER) membrane bilayer, from where it is distributed to other membrane compartments by vesicular transport or by cytosolic transfer proteins. Dephosphorylation and phosphorylation of PIs are rapid processes and carried out by PI phosphatases and kinases, respectively, that render PIs highly interconvertible. Most of these enzymes target a specific position within the inositol ring, although some may accept multiple PIs as substrate. In the latter case, substrate specificity could be defined by compartment-specific regulatory proteins and lipid-substrate availability (De Matteis and Godi 2004; Krauss and Haucke 2007). The local balance, distribution and regulation of PI species is determined by the organelle specific subset of PI metabolizing enzymes (Balla 2013) (For a comprehensive overview see Table 1). Their membrane recruitment and sub-compartmental targeting is mostly regulated by PI-binding domains, membrane-anchors, such as reversible palmitoylation, or peripheral membrane proteins, including small Rab GTPases and transport coat components.

A variety of cellular processes and pathways are linked to PIs and PI turnover. To elaborate on all their functions in detail is beyond the scope of this introduction. Instead I will focus on their role in intracellular and exocytic membrane trafficking, with a particular focus on $\mathrm{PI}(3) \mathrm{P}$ and phosphatidylinositol-4-phosphate (PI(4)P), and the corresponding enzymes implicated in their turnover, namely type II PI 4-kinases and PI(3)P hydrolyzing phosphatases of the myotubularin family.

\section{Table 1: PI metabolizing enzymes}

An overview of mammalian PI kinases and phosphatases is given with the preferred substrate and intracellular localization of the enzyme. PM (plasma membrane); TGN (trans-Golgi network); E (endosomal compartment); GC (Golgi complex); ER (endoplasmatic reticulum); SV (secretory vesicles); N (nucleus), LE (late endosomes), L (lysosomes)

\begin{tabular}{|c|c|c|c|}
\hline Enzymatic activity & Isoforms & Predominant substrate & Localization \\
\hline \multicolumn{4}{|l|}{ PI kinases } \\
\hline \multirow[t]{3}{*}{ PI 3-kinase } & $P 100 \alpha-\delta$ & $\mathrm{PI}(4,5) \mathrm{P}_{2}$ & $\mathrm{PM}$ \\
\hline & $\mathrm{PI} 3 \mathrm{KC} 2 \alpha, \beta, \gamma$ & PI, PI(4)P & PM, TGN, GC, E, SV \\
\hline & hVps34 & PI & $\mathrm{E}$ \\
\hline \multirow[t]{2}{*}{ PI 4-kinase } & PI4KII $\alpha, \beta$ & PI & PM, E, GC, SV \\
\hline & PI4KIII $\alpha, \beta$ & PI & $\mathrm{PM}, \mathrm{GC}, \mathrm{ER}, \mathrm{N}$ \\
\hline PI 5-kinase & PIKfyve & PI & $\mathrm{LE}$ \\
\hline PIP4K & PIP4K $\alpha, \beta, \gamma$ & $\mathrm{PI}(5) \mathrm{P}$ & $\mathrm{PM}$ \\
\hline \multirow[t]{3}{*}{ PIP5K } & PIKfyve & $\mathrm{PI}(3) \mathrm{P}$ & LE \\
\hline & PIP5K $\alpha, \beta, \gamma$ & PI(4)P & PM \\
\hline & PIP5KL1 & Inactive & $\mathrm{PM}$ \\
\hline $\begin{array}{l}\text { PI phosphatases } \\
\text { 1-phosphatase }\end{array}$ & IPPase & $\mathrm{I}(1,4) \mathrm{P}_{2}$ & \\
\hline
\end{tabular}




\begin{tabular}{|c|c|c|c|}
\hline Enzymatic activity & Isoforms & Predominant substrate & Localization \\
\hline \multirow[t]{4}{*}{ 3-phosphatase } & MTM1, MTMR1-4,6-8,14 & $\mathrm{PI}(3) \mathrm{P}, \mathrm{PI}(3,5) \mathrm{P}_{2}, \mathrm{I}(1,3) \mathrm{P}_{2}$ & E, LE, PM \\
\hline & MTMR5, MTMR9-13 & Inactive & \\
\hline & PTEN1,TPTE2 & $\mathrm{PI}(3,4) \mathrm{P}_{2}, \mathrm{PI}(3,5) \mathrm{P}_{2}, \mathrm{PI}(3,4,5) \mathrm{P}_{3}$ & PM, GC \\
\hline & PTPRQ & various PIs & - \\
\hline \multirow[t]{4}{*}{ 4-phosphatase } & Sac1,Sac2 (INPP5F), Sac3 (Fig4) & $\mathrm{PI}(4) \mathrm{P}$ & $\mathrm{E}, \mathrm{ER}, \mathrm{GC}$ \\
\hline & synaptojanin 1,2 & various PIs, i.e. $\mathrm{PI}(4,5) \mathrm{P}_{2}$, $\mathrm{PI}(4) \mathrm{P}$ & - \\
\hline & Type I, II (INPP4A/4B) & $\mathrm{PI}(3,4) \mathrm{P}_{2}$ & PM, E \\
\hline & TMEM55A, 55B & $\mathrm{PI}(4,5) \mathrm{P}_{2}$ & LE, L \\
\hline \multirow[t]{10}{*}{ 5-phosphatase } & SHIP 1,2 & $\mathrm{PI}(3,4,5) \mathrm{P}_{3}$ & $\mathrm{PM}$ \\
\hline & synaptojanin 1,2 & various PIs, i.e. $\mathrm{PI}(4,5) \mathrm{P}_{2}$ & SV, PM \\
\hline & OCRL & $\mathrm{PI}(4,5) \mathrm{P}_{2}$ & TGN, E \\
\hline & PIPP (INPP5J) & $\mathrm{PI}(4,5) \mathrm{P}_{2}, \mathrm{PI}(3,4,5) \mathrm{P}_{3}$ & PM \\
\hline & SKIP (INPP5K) & $\mathrm{PI}(4,5) \mathrm{P}_{2}, \mathrm{PI}(3,4,5) \mathrm{P}_{3}$ & $\mathrm{ER}, \mathrm{PM}$ \\
\hline & 72kD 5-phosphatase (INPP5E) & $\mathrm{PI}(3,5) \mathrm{P}_{2}, \mathrm{PI}(4,5) \mathrm{P}_{2}, \mathrm{PI}(3,4,5) \mathrm{P}_{3}$ & GC \\
\hline & 5-phosphatase II & $\mathrm{PI}(4,5) \mathrm{P}_{2}, \mathrm{PI}(3,4,5) \mathrm{P}_{3}$ & Mi, PM \\
\hline & 5-phosphatase IV & $\mathrm{PI}(3,4,5) \mathrm{P}_{3}$ & \\
\hline & 5PTase type I (INPP5A) & $\mathrm{I}(1,4,5) \mathrm{P}_{3}$ & \\
\hline & 5PTase typeII & $\mathrm{I}(1,4,5) \mathrm{P}_{3}, \mathrm{I}(1,3,4,5) \mathrm{P}_{4}$ & \\
\hline
\end{tabular}

\subsection{Membrane traffic is regulated by phosphoinositides}

\subsubsection{Phosphoinositides serve as spatio-temporal landmarks}

The specific distribution of different PI species between intracellular membranes contributes to defining organelle identity (De Matteis and Godi 2004). The metabolism and interconversion of the different PI species needs to parallel the maturation of organelles within the exocytic, endocytic and degradative pathways (Figure 1) (Krauss and Haucke 2007).

Whereas the plasma membrane (PM) is enriched in $\mathrm{PI}(4) \mathrm{P}$ and its derivatives phosphatidylinositol-4,5-bisphosphate $\quad\left(\mathrm{PI}(4,5) \mathrm{P}_{2}\right)$ and phosphatidylinositol-3,4,5trisphosphate $\left(\mathrm{PI}(3,4,5) \mathrm{P}_{3}\right)$, the early endosomal compartment is enriched in $\mathrm{PI}(3) \mathrm{P}$. In addition to early endosomes the internal vesicles of multivesicular bodies (MVB) show a high content of PI(3)P while the limiting membrane of MVBs and the late endosomal compartment are enriched in phosphatidylinositol-3,5-bisphosphate $\left(\mathrm{PI}(3,5) \mathrm{P}_{2}\right)$ (Lindmo and Stenmark 2006). The Golgi complex, in particular the TGN, is characterized by PI(4)P, a PI that is of particular importance for the integrity of the Golgi, largely through its involvement in AP-1and GGA-dependent vesicle formation at the TGN (Wang, Sun et al. 2007). So far, the subcellular distribution of PI(5)P remains poorly understood, although a regulatory role in autophagosome biogenesis (Vicinanza, Korolchuk et al. 2015), cell migration and enzymatic activity of $\mathrm{PI}(3,5) \mathrm{P}_{2}$-metabolizing enzymes was reported (Haugsten, Oppelt et al. 2013; Oppelt, Lobert et al. 2013). 
Intracellular membrane identity is further defined by the subcellular distribution of GTPases of the Arf and Rab families (Behnia and Munro 2005). These proteins can regulate localization and/ or enzymatic activity of PI kinases and phosphatases in their GTP- and membrane-bound state, and thus provide spatiotemporal regulation of PI composition and mechanisms for positive and negative feed back loops.

Membrane recruitment of PI effector proteins is mediated via PI binding domains (Lemmon 2008). However, as PI-protein interactions are of moderate affinity, efficient recruitment often requires coincidence detection of a specific small GTPase, PIs (Di Paolo and De Camilli 2006) and in some cases additional protein-protein interactions, i.e. during clathrin-mediated endocytosis (Godi, Pertile et al. 1999; Behnia and Munro 2005; Lemmon 2008). This fundamental concept applies to transport carrier biogenesis at various subcellular compartments, despite the diversity of coat proteins (Santiago-Tirado and Bretscher 2011). Therefore, the spatio-temporal regulation of PI turnover is crucial in intracellular transport including membrane organization, deformation, assembly of adaptor protein complexes and fission and fusion of membrane-derived vesicles.

\subsubsection{PI(4,5) $P_{2}$-to-PI(3)P conversion in endocytic membrane traffic}

PI content and conversion during CME and on PM-derived endocytic vesicles are essential for progression through the endocytic pathway from a $\mathrm{PI}(4) \mathrm{P}$ - and $\mathrm{PI}(4,5) \mathrm{P}_{2}$-positive PM to a PI(3)P-enriched early endosome. This conversion is assured by the spatio-temporally controlled, sequential action of PI-kinases and -phosphatases and recruitment of PI-specific adaptor complexes (Posor, Eichhorn-Grunig et al. 2015).

The PI identity of the inner leaflet of the PM is characterized by the local enrichment of $\mathrm{PI}(4) \mathrm{P}$ and $\mathrm{PI}(4,5) \mathrm{P}_{2}$. $\mathrm{PM}$ hot spots of $\mathrm{PI}(4,5) \mathrm{P}_{2}$ are synthesized to modulate a variety of processes emanating at the cell surface such as cell signaling, organization of the cytoskeleton and endocytosis. The plasma membrane PI(4)P pool is generated by $\mathrm{PI} 4 \mathrm{~K} 3 \alpha$ (Bojjireddy, Botyanszki et al. 2014) and serves as a substrate for local PI(4,5) $\mathrm{P}_{2}$ synthesis by PI(4)P-5kinases (PIP5Ks). Besides being a precursor for $\mathrm{PI}(4,5) \mathrm{P}_{2}$, recent studies indicate that $\mathrm{PI}(4) \mathrm{P}$ contributes to the polyanionic lipid pool that defines the PM, additionally to, but independent of $\mathrm{PI}(4,5) \mathrm{P}_{2}$ (Hammond, Fischer et al. 2012). Clathrin coated pit (CCP) nucleation, although dependent on the general PM PI(4,5) $\mathrm{P}_{2}$ content (Zoncu, Perera et al. 2007), is driven by local synthesis of $\mathrm{PI}(4,5) \mathrm{P}_{2}$ by $\mathrm{PIP} 5 \mathrm{~K}$ type $\mathrm{I}$. The most prominent $\mathrm{PI}(4,5) \mathrm{P}_{2}$-specific clathrin adaptor is the tetrameric adaptor protein AP-2 and cargo-AP-2-complexes stimulate PIP5K activity (Krauss, Kukhtina et al. 2006). While $\mathrm{PI}(4,5) \mathrm{P}_{2}$-synthesis is restricted to CCP 
initiation, $\mathrm{PI}(4,5) \mathrm{P}_{2}$ 5-phosphatases and 4-phosphatases such as synaptojanin (Perera, Zoncu et al. 2006) or SHIP2 (Nakatsu, Perera et al. 2010) are recruited during CCP maturation. At the same time $\mathrm{PI}(3,4) \mathrm{P}_{2}$-synthesis from $\mathrm{PI}(4) \mathrm{P}$ is initiated by clathrin-dependent recruitment of PI 3-kinase type II $\alpha$ (PI3KC2 $\alpha$ ) (Posor, Eichhorn-Gruenig et al. 2013). Thus, this is the initial step in acquiring early endosomal 3-phosphate identity. Recruitment of the $\operatorname{PI}(3,4) \mathrm{P}_{2}$ specific adaptor proteins SNX9/ SNX18 (Posor, Eichhorn-Gruenig et al. 2013) drives constriction of the budding vesicle neck, while $\mathrm{PI}(4,5) \mathrm{P}_{2}$ is required for dynamin-dependent membrane fission (Vallis, Wigge et al. 1999). 4'- and 5'-dephosphorylation of $\mathrm{PI}(4,5) \mathrm{P}_{2}$ by synaptojanin (Verstreken, Koh et al. 2003) or the oculocerebrorenal syndrome of Lowe (OCRL) (Nandez, Balkin et al. 2014) favours dissociation of AP-2 and disassembly of the clathrin coat. Further, the $\mathrm{PI}(3,4) \mathrm{P}_{2}$-specific inositol-4-phosphatase INPP4B, an early endosomal Rab5 effector, might be required for $\mathrm{PI}(3,4) \mathrm{P}_{2}$-removal from CCVs and thereby adds to generation of the early endosomal PI(3)P pool (Shin, Hayashi et al. 2005; Posor, Eichhorn-Grunig et al. 2015). These steps thus help to restrict $\mathrm{PI}(4,5) \mathrm{P}_{2}$ and $\mathrm{PI}(3,4) \mathrm{P}_{2}$ to the plasma membrane (Erdmann, Mao et al. 2007). To assure dephosphorylation of $\mathrm{PI}(4,5) \mathrm{P}_{2}$ (Verstreken, Koh et al. 2003; Erdmann, Mao et al. 2007) and PI(3,4) $\mathrm{P}_{2}$ as well as synthesis of PI(3)P (Christoforidis, Miaczynska et al. 1999; Murray, Panaretou et al. 2002) these PI 5phosphatases and PI 4-phospatases are included in a Rab5 complex comprising its GEF rabaptin-5-rabex-5 and class III PI 3-kinase Vps34. Recently, the PI(4)P 4-phosphatase Sac2 was implicated in endocytic trafficking and recycling, also an early endosomal Rab5 effector that associates with OCRL and INPP4B (Hsu, Hu et al. 2015; Nakatsu, Messa et al. 2015). Thus, this Rab5 complex is essential for accumulation and maintenance of $\mathrm{PI}(3) \mathrm{P}$ on early endosomal membranes, while at the same time depleting 4- and 5-phosphorylated PI species. Thereby, this enzymatic cascade generates early endosomal PI(3)P identity.

\subsubsection{Endosomal sorting occurs via tubular cargo-enriched subdomains}

Sorting endosomes are a subpopulation of early endosomes designated to discriminate between cargo-specific transport routes. As sorting endosomes are of early endosomal origin they are enriched in $\mathrm{PI}(3) \mathrm{P}$ and most cargo-specific sorting adaptors are recruited by coincidence detection of PI(3)P, small Rab GTPases and/ or additional cargo- and transport route specific cues. Herein, tubular cargo-enriched subdomains discriminate between transport to late endosomes, the PM or TGN (Hutagalung and Novick 2011). Tubule formation and cargo-sorting is coordinated by various members of the sorting nexin (SNX) family (Figure 3) (Cullen 2008). To integrate these functions, most SNXs contain a BAR 
domain, which forms rigid dimers that bind to positively curved membranes, a PI-specific Phox-homology (PX) domain and/ or recognize sorting motifs, i.e. Asn-Pro-x-Tyr (NPxY) motifs or PDZ (post synaptic density) - ligands, within the cytoplasmic domain of cargo proteins. Although SNX-BAR proteins can stabilize and drive tubule formation in vitro (Carlton, Bujny et al. 2004), it remains unclear if SNX-BAR proteins are targeted to preexisting, tubular, PI-enriched endosomal subdomains or remodel the endosomal membrane themselves.

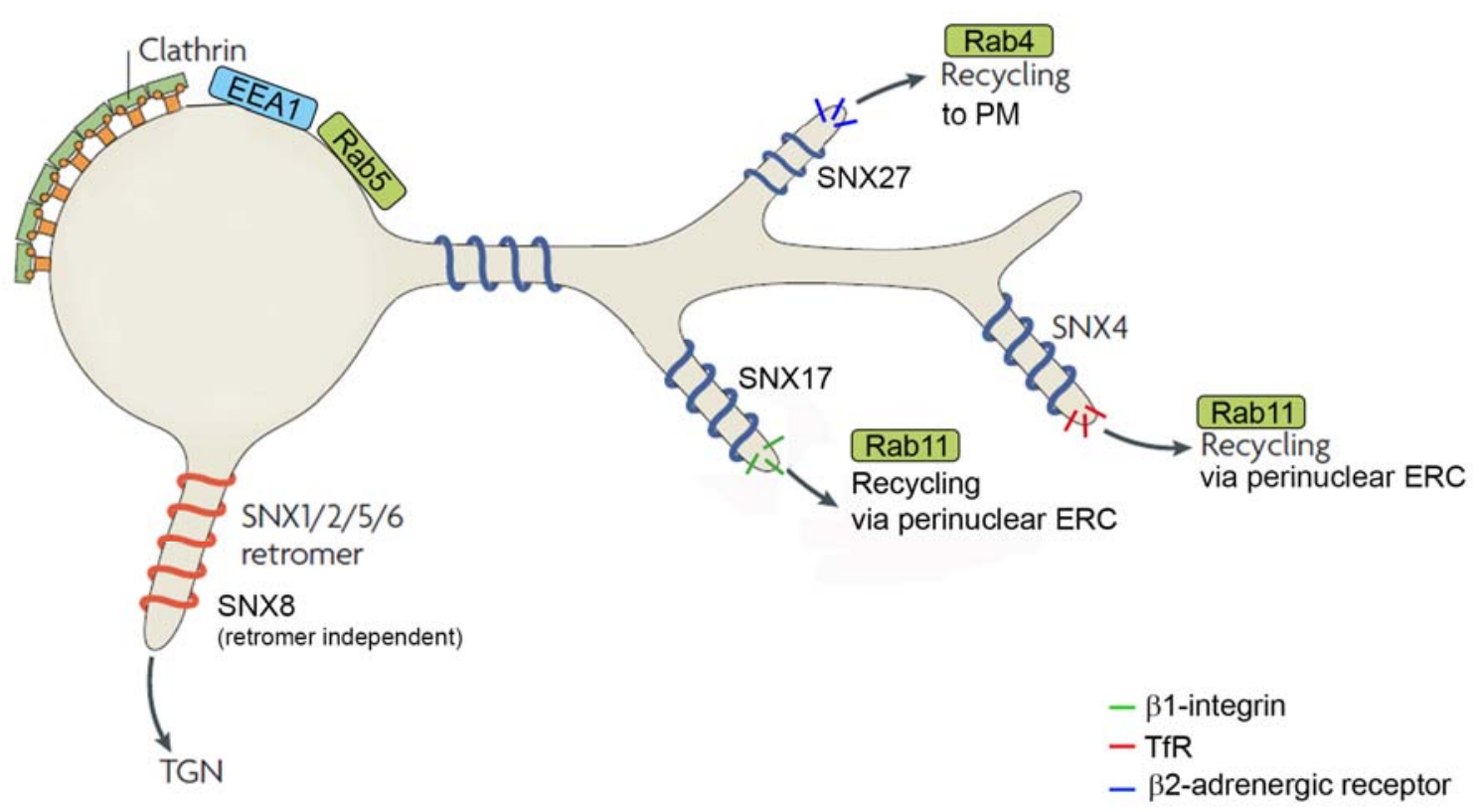

Figure 3: Tubular cargo-enriched subdomains at the early sorting endosome are formed by SNXs

Tubule formation at the Rab5- and EEA1-positive early endosome depends on PI(3)P-binding SNXs and allows cargo segregation and sorting for transport to the trans-Golgi-network (TGN), the plasma membrane (PM) or the perinuclear, endocytic recycling compartment (ERC). Modified from (Cullen 2008) .

Rab as well as PI conversion represent hallmarks of endosomal maturation and include switching of early endosomal Rab5 to Rab4 in fast endocytic recycling (de Renzis, Sonnichsen et al. 2002), to Rab7 during late endosomal progression (Takatori, Tatematsu et al. 2015) or to Rab11 in slow recycling via the perinuclear recycling compartment. PI(3)Pdependent endosomal sorting via SNXs includes: SNX27-retromer mediated recycling of PDZ-ligand-containing cargo proteins (Temkin, Lauffer et al. 2011); The SNX1/2/5/6retromer-mediated or SNX8-mediated endosome-to-TGN trafficking (Dyve, Bergan et al. 2009); SNX4- or SNX 17-mediated recycling of TfR (Traer, Rutherford et al. 2007) and $\beta 1$ integrin (Steinberg, Heesom et al. 2012), respectively. Since muscle fibers from XLCNMpatients display endosomal accumulation of $\beta 1$-integrins, we addressed its endosomal sorting 
and exocytic transport. In addition, transferrin receptor (TfR) is constantly sorted and recycled along a similar route and often used exemplarily to study both Rab4- and Rab11-mediated recycling.

TfR-sorting and tubule formation by SNX4 at the early endosome occurs as endosomes change their identity from early/ Rab4- to recycling/ Rab11-positive endosomes (van Weering, Verkade et al. 2012). By binding to KIBRA SNX4 links PI(3)P-enriched early endosomes to dynein motors and promotes carrier transport to the junxtanuclear recycling compartment and subsequent $\mathrm{PI}(4) \mathrm{P}$-dependent carrier release from microtubule tracks (Traer, Rutherford et al. 2007). The plus-end directed motor protein Kif16b, also known as SNX23, is targeted via its PI(3)P-binding PX-domain to early endosomes, dependent on Rab5 and Vps34 activity. Thus, Kif16b is required for endosome positioning and efficient early endosomal recycling (Hoepfner, Severin et al. 2005).

Recycling of cargo proteins that contain NPxY-sorting motifs including $\beta 1$-integrin (Bottcher, Stremmel et al. 2012; Steinberg, Heesom et al. 2012), LDLR (Burden, Sun et al. 2004) and LRP1 (Donoso, Cancino et al. 2009) in polarized and neuronal cells is mediated by SNX17 (Czubayko, Knauth et al. 2006). Efficient SNX17-mediated $\beta 1$-integrin recycling via Rab11-positive recycling endosomes en route to the cell surface prevents ubiquitylation of $\alpha$ and $\beta$-integrin cytodomains and rapid lysosomal degradation.

So far, a clear correlation between Rab- and PI-conversion in endosomal transport was only shown for the Rab5-to-Rab7-switch, accompanied by $\mathrm{PI}(3) \mathrm{P}$ to $\mathrm{PI}(3,5) \mathrm{P}_{2}$ conversion (Figure 4). This step requires the PI and PI(3)P-specific 5-kinase PIKfyve, the mammalian homologue of yeast Fab1 (Gary, Wurmser et al. 1998). PIKfyve is found in a complex with the active PI(3,5) $\mathrm{P}_{2}$ 5-phosphatase mammalian Sac3 (Ikonomov, Sbrissa et al. 2009) or with yeast Fig4, which is scaffolded by ArPIKfyve in mammals (yeast Vac14) (McCartney, Zhang et al. 2014). PIKfyve localizes via its FYVE-domain to PI(3)P-positive endosomes. Positive regulation of its activity by Sac3 (Ikonomov, Sbrissa et al. 2003; Sbrissa, Ikonomov et al. 2007) assures balanced $\mathrm{PI}(3,5) \mathrm{P}_{2}$ synthesis together with a high turnover rate. According to the role of its lipid product $\mathrm{PI}(3,5) \mathrm{P}_{2}$ loss of PIKfyve impairs retrograde endosome-to-Golgi transport and degradative sorting of i.e. EGFR (de Lartigue, Polson et al. 2009).

\section{Figure 4: Endosomal trafficking is regulated by PI 3-kinases and phosphatases}

Schematic representation of the subcellular distribution of $\mathrm{PI}(3) \mathrm{P}$ and $\mathrm{PI}(3,5) \mathrm{P}_{2}$ as well as their metabolizing enzymes. PI 3-kinase are shown in green, PI 3-phosphatases are shown in red. EE: early endosome; ER: endoplasmic reticulum; GC: Golgi complex; LE: late endosome; MLV: multi vesicular body; N: nucleus; RE: recycling endosome; SE: sorting endosome; TGN: trans-Golgi-network; Tfn: Transferrin. Modified from (Hnia, Vaccari et al. 2012). 


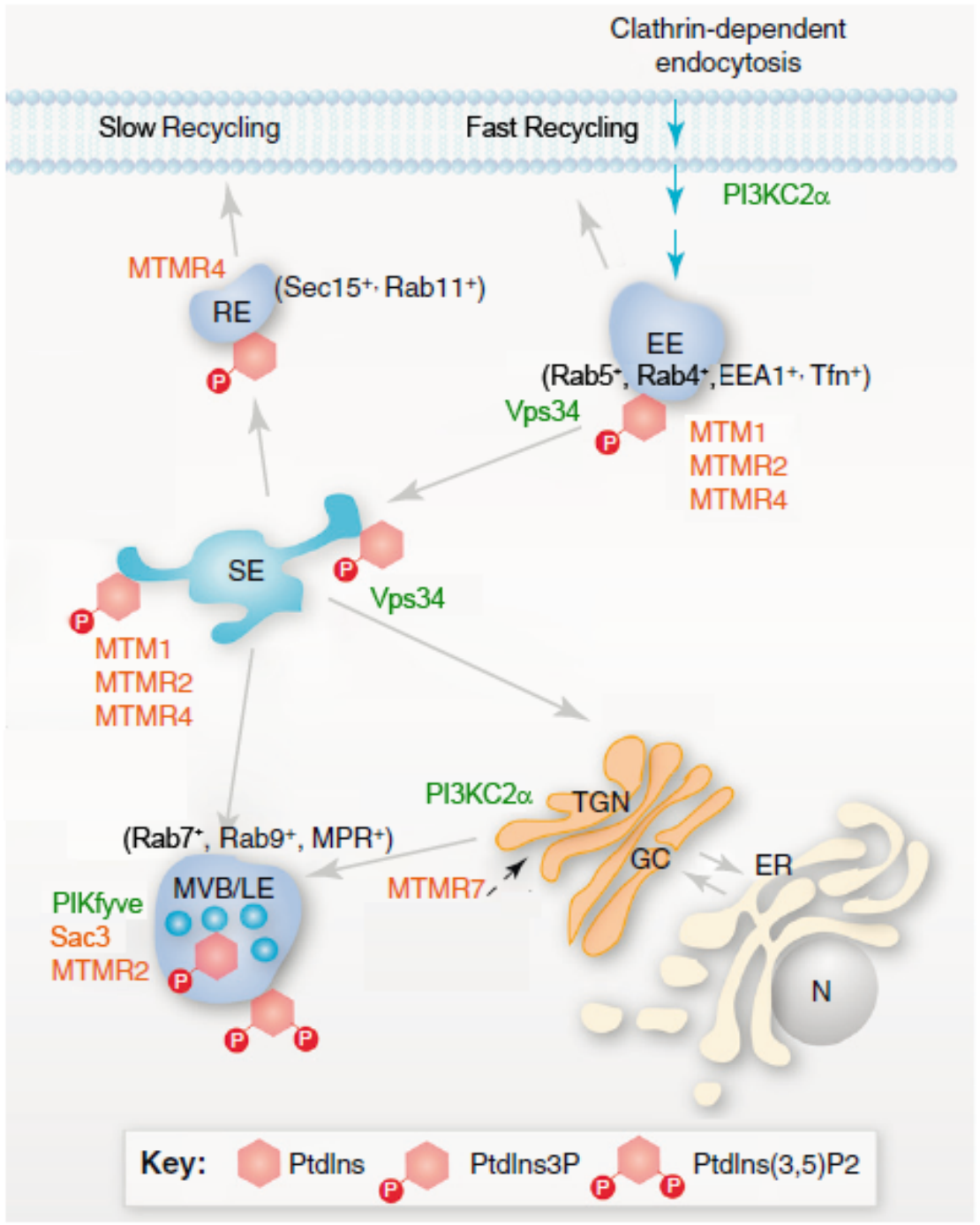

\subsubsection{Exocyst tethering in endosomal exocytosis is regulated by endosomal and cell surface determinants}

The delivery of cargo proteins to the cell surface can occur along a variety of transport routes. These routes are regulated by common underlying principles, including transport along microtubule or actin tracks, tethering complexes that allow targeted secretion and subsequent SNARE-mediated membrane fusion. One such tether is the exocyst, an evolutionary conserved, octameric complex that targets recycling endosomes or secretory vesicles to the PM prior to fusion (Figure 5). This process is of crucial importance as it is involved in cytokinesis, cell migration, ciliogenesis and polarized membrane traffic (Wu and Guo 2015).

Exocyst localization depends on PIs and small GTPases: PM localization of the Sec3 and Exo70 subunits is regulated by $\mathrm{PI}(4,5) \mathrm{P}_{2}$ (He, Xi et al. 2007). Interestingly, Sec3 and Exo70 were speculated to localize to both PM and secretory vesicles (Boyd, Hughes et al. 2004). Exocyst assembly on endosomes is driven by the Sec15 subunit which is a Rab11 
effector (Zhang, Ellis et al. 2004) that directly interacts with the exocyst subunits Sec10, Exo84 and Exo70. Consequently, loss of exocyst (Sec15 or Exo70) or Rab11 perturbs endosomal recycling (Takahashi, Kubo et al. 2012). Further, exocyst subunits Sec6 and Sec8 were shown to directly associate with the SNARE-machinery (Kubo, Kobayashi et al. 2015) and Sec6 facilitates SNARE-complex assembly (Dubuke, Maniatis et al. 2015). Thereby, targeting of the vesicular exocyst complex to exocytic sites is coupled with SNARE-mediated PM fusion.

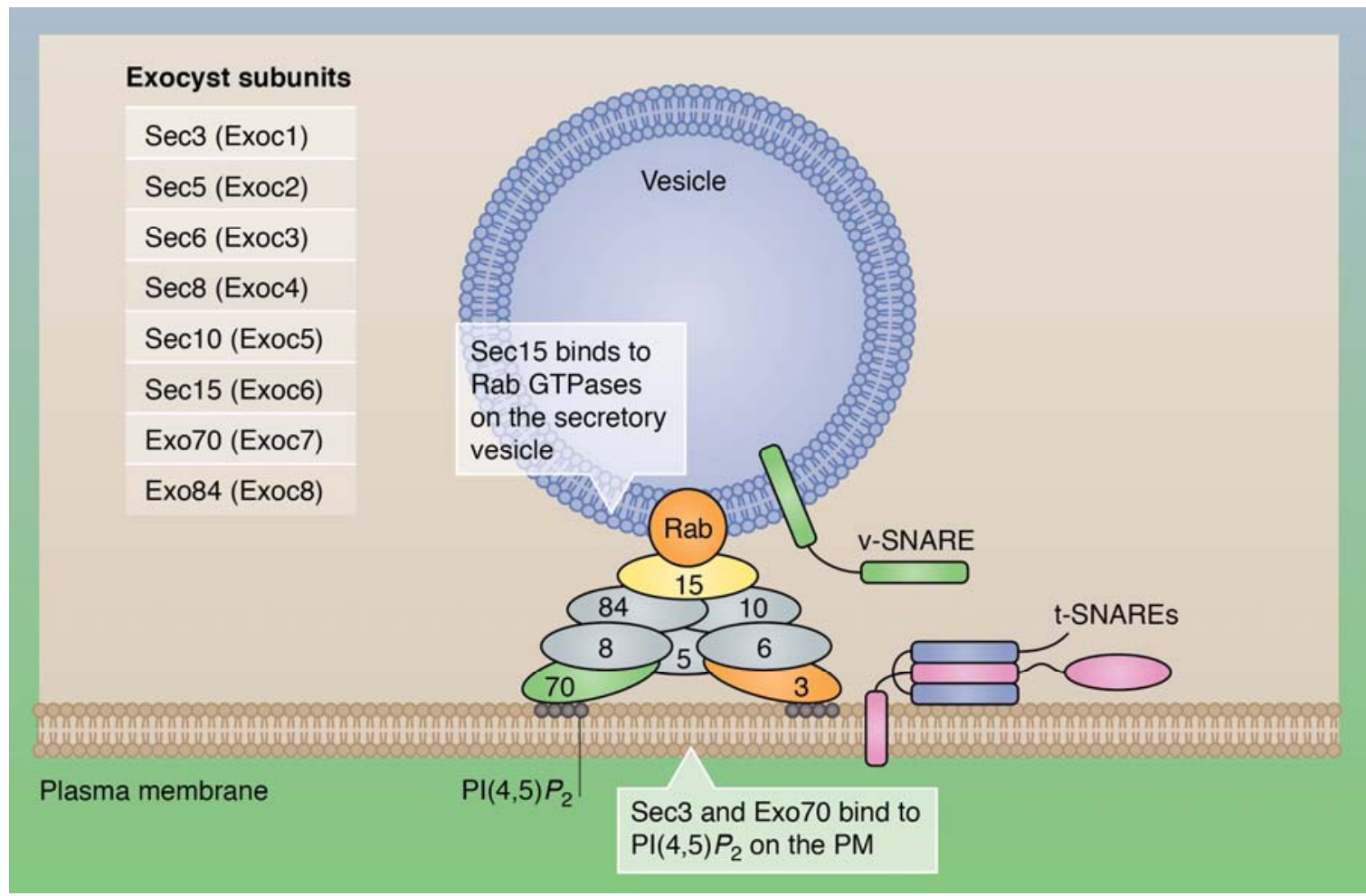

Figure 5: Exocyst assembly and vesicle tethering

Exocyst subunit Sec15 assembles the octameric complex on recycling endosomes or secretory vesicles, while Exo70 and Sec3 target these vesicle to the plasma membrane by binding to PI(4,5) $\mathrm{P}_{2}$. Association with vSNAREs, i.e.VAMP2/3, t-SNAREs, i.e. Syntaxin 13, and SNAP23/25 couples exocyst tethering to plasma membrane fusion (Wu and Guo 2015).

The PM pool of PI $(4,5) \mathrm{P}_{2}$ for exocyst targeting is presumably synthesized by PIP5K type I: Association of PIP5KI with Exo70 together with $\mathrm{PI}(4,5) \mathrm{P}_{2}$ synthesis at the PM directs E-cadherin clustering, adherence junction formation and epithelial cell polarity (Xiong, Xu et al. 2012), while in migrating cells PM PI(4,5) $\mathrm{P}_{2}$ generated by PIP5KI coordinates exocystmediated polarized surface delivery of integrins (Thapa, Sun et al. 2012; Sun, Thapa et al. 2013). However, lipid requirements on exocytic vesicle for assembling the fusion machninery remain unclear. Purified chromaffin granules and VAMP2-positive synaptic vesicles contain PI 4-kinase type II $\alpha$ (PI4K2 $\alpha$ ) (Guo, Wenk et al. 2003). In non-neuronal cells, PI4K2 $\alpha$ 
localizes to early and recycling endosomes via palmitoylation. PI4K2 $\alpha$ and its lipid product PI(4)P regulate endosomal sorting of the vesicular (v) -SNARE VAMP3, required for TfR recycling (Galli, Chilcote et al. 1994; Jovic, Kean et al. 2014). In yeast, secretory vesicles are formed at PI(4)P- and Ypt32p (homologues to mammalian Rab11)-enriched subdomains at the TGN, followed by a Ypt32p-to-Sec4 (homologues to mammalian Rab8), switch as additional exocyst subunits are assembled (Mizuno-Yamasaki, Medkova et al. 2010). Together, these data implicate a vesicular PI(4)P pool in exocytic membrane traffic.

\subsection{Myotubularins are a family of PI 3-phosphatases}

The myotubularin family encompasses 15 members in humans, MTM1 and MTMR1-14 (Hnia, Vaccari et al. 2012). According to their catalytic signature, the Cys- $\mathrm{X}_{5}$-Arg motif, they belong to the family of protein tyrosine phosphatases/ dual specific phosphatases (PTP/DSP), although they specifically dephosphorylate $\mathrm{PIs}\left(\mathrm{PI}(3) \mathrm{P}\right.$ and $\left.\mathrm{PI}(3,5) \mathrm{P}_{2}\right)$ at the 3'-position, with the exception that MTMR7 predominantly accepts $\mathrm{I}(1,3) \mathrm{P}_{2}$ as substrate.

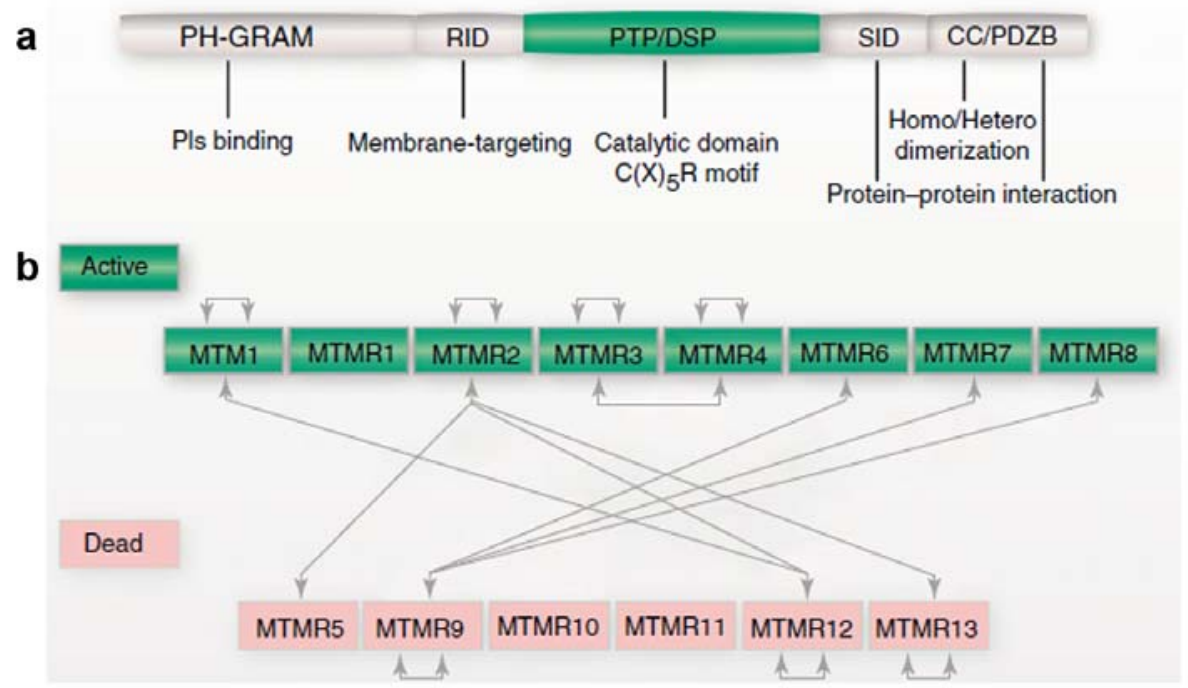

Figure 6: Domain structure and 'active/dead' combinations of the myotubularin family

(a) Common domain architecture of the myotubularin family. PH (Pleckstrin-homology), GRAM (glycosyltransferase, Rab-like GTPase activator and myotubularin), RID (Rac-induced recruitment domain), SID (SET-interacting domain), CC (coiled-coil), PDZB (PDZ-binding motif). (b) Homo- and heterodimeric interactions within the myotubularin family (Hnia, Vaccari et al. 2012).

Myotubularins comprise active and inactive phosphatases, the latter lacking the conserved cysteine within the $\mathrm{CX}_{5} \mathrm{R}$-motif. Homo- and heterodimerisation, mostly occurring 
between active and inactive myotubularins, regulates localization as well as enzymatic activity, allowing for spatiotemporal regulation by distinct 'active/dead' combinations (Figure 6). Further, myotubularins share a common domain structure: (a) PH-GRAM (glycosyltransferase, Rab-like GTPase activator and myotubularin) domain, (b) RID (Racinduced recruitment domain), (c) PTP domain and (d) SID (SET-interacting domain).(a) The PH-GRAM domain mediates PI binding and contributes to subcompartment-specific targeting as well as (b) RID, which was identified as membrane targeting domain of MTM1 to Rac1induced membrane ruffles at the PM (Laporte, Blondeau et al. 2002). While (c) the PTP domain contains the $\mathrm{CX}_{5} \mathrm{R}$ motif, (d) SID, an integral part of the PTP domain (Begley, Taylor et al. 2003), was implicated in protein-protein interactions. Additionally, most MTMs contain a coiled-coil (CC) domain, critical for homo- and heterodimerisation, and a PDZ-binding motif (PDZB), involved in protein-protein-interactions (Figure 6).

\subsubsection{MTM1 regulates endosomal PI(3)P turnover and membrane dynamics}

Mammalian MTM1 and its homologues in various other species were proposed to balance PI-levels within the endosomal system and thereby regulate membrane dynamics and traffic. According to its $\mathrm{PI}(3) \mathrm{P}$ and $\mathrm{PI}(3,5) \mathrm{P}_{2}$ 3-phosphatase activity, MTM1 localizes to early and late endosomes. Recruitment and PI turnover is thought to be regulated by Vps34 (Figure 4) (Cao, Laporte et al. 2007) and by the phosphatase-inactive MTMR12 (Nandurkar, Layton et al. 2003). Targeted recruitment of MTM1 to early endosomes and thereby PI(3)P depletion enhances microtubule-dependent endosome tubulation (Fili, Calleja et al. 2006) during endosomal recycling. However, based on other studies local PI(3)P-turnover was suggested to be a crucial determinant for tubule formation (Carpentier, N'Kuli et al. 2013). In Drosophila melanogaster the MTM1 and MTMR2 ortholog mtm regulates a PI(3)P pool synthesized by class II and/ or class III PI 3-kinases, required for endo-lysosomal membrane homeostasis and cortical remodeling in immune cells (Velichkova, Juan et al. 2010). Upon loss of mtm in muscle fibers, $\beta 1$-integrin accumulates in $\mathrm{PI}(3) \mathrm{P}$-enriched endosomes leading to defects in integrin-mediated myofiber attachment. In Saccharomyces cerevisae the sole myotubularin, Ymrlp, cooperates with synaptojanin-like PI-phosphatase Sj13p in regulation of PI(3)P levels. Yeast double-deletion strains accumulate $\mathrm{PI}(3) \mathrm{P}$ on vacuolar membranes causing misslocalization of PI(3)P effectors, defective sorting of vacuolar hydrolases and vacuole fragmentation (Parrish, Stefan et al. 2004). Taken together, these data indicates that balancing $\mathrm{PI}(3) \mathrm{P}-$ level is essential for endomembranes traffic. 
MTM1 was originally identified as the gene mutated in X-linked centronuclear myopathy (XLCNM) (as discussed in section 1). Since muscle contraction and organization relies on the cytoskeleton, it is interesting to note that MTM1 directly regulates desmin intermediate filaments (IF) in vitro and in vivo. Thus, XLCNM-causing mutations in the desmin-binding interface of MTM1 abolish MTM1-desmin complex formation leading to altered IFs and mitochondrial dysfunction (Hnia, Tronchere et al. 2011). Although the primary and most striking phenotype of MTM1-loss-of-function affects muscle fibers, patients with a milder form of XLCNM carrying MTM1 missense mutations develop additional symptoms such as liver dysfunction (Herman, Finegold et al. 1999). Thus, MTM1 function might not be restricted to muscles. Further, functional redundancy in vivo between MTM1 and MTMR1 or MTMR2 might be ruled out, as MTM1 and MTMR2 are inversely regulated during myogenesis (Kim, Taylor et al. 2002), predominantly expressed in distinct, but different tissue and do not compensate for MTM1-deficiency in muscle fibers (Al-Qusairi, Prokic et al. 2013).

\subsubsection{Other myotubularin family members contribute to endosomal PI(3)P turnover}

The majority of myotubularins remains poorly characterized. However, some members of this 3-phosphatase family were previously implicated in endosomal $\mathrm{PI}(3) \mathrm{P}$ and $\mathrm{PI}(3,5) \mathrm{P}_{2}$ homeostasis, such as MTMR2, MTMR4 and presumably due to MTM1-homology MTMR1 (Figure 4). Thus, this section focuses on the latter myotubularins and their role in endosomal membrane traffic.

MTMR1 is the closest homologue of MTM1 and originated from gene duplication early in evolution before separation of human and mouse lineage (Kioschis, Wiemann et al. 1998). MTMR1 is a PI(3)P 3-phosphatase, ubiquitously expressed and regulated via alternative splicing especially during myogenesis with differences in adult and fetal isoforms (Buj-Bello, Furling et al. 2002). Although no function has been assigned to MTMR1 so far, aberrant splicing of MTMR1 is associated with congenital myotonic dystrophy (cDM1) (Santoro, Modoni et al. 2010), which is pathologically similar to XLCNM. Since phenotypic changes in MTM1-null mice are mostly restricted to muscle fibers, without compensatory upregulation of MTMR1 (Al-Qusairi, Prokic et al. 2013), MTMR1 might substitute for MTM1 in other tissues.

MTMR2 was originally identified as the gene mutated in Charcot-Marie-Tooth disease type 4B1 (CMT4B1). The mutations identified so far abolish enzymatic activity and/ or give 
rise to truncations. CMT4B1 is a peripheral neuropathy that is caused by myelination defects. Accordingly, MTMR2 is highly expressed in neurons and Schwann cells. Schwann cellspecific gene knockout causes myelin outfoldings that resemble CMT4B1 symptoms, preferentially arising at paranodal regions (Bolino, Bolis et al. 2004). Interestingly, these regions are characterized by a high degree of membrane remodeling. MTMR2 preferentially binds to $\mathrm{PI}(3,5) \mathrm{P}_{2}$ via its $\mathrm{PH}$-domain (Begley, Taylor et al. 2003), predominantly accepts $\mathrm{PI}(3,5) \mathrm{P}_{2}$ as a substrate and localizes to early and late endosomes (Figure 4) (Franklin, Taylor et al. 2011). Through association with Vps34 MTMR2 is thought to balance endosomal $\mathrm{PI}(3) \mathrm{P}$ and $\mathrm{PI}(3,5) \mathrm{P}_{2}$ levels and regulates early to late endosomal trafficking (Cao, Backer et al. 2008). In addition, MTMR2 recruits the PI(3)P- and PI(3,5) $\mathrm{P}_{2}$-effector RME-8 (receptor mediated endocytosis 8) and, thus, links PI-conversion to retromer-dependent endosomal tubulation (Cao, Backer et al. 2008; Xhabija, Taylor et al. 2011). In Schwann cells, MTMR2 interacts with Discs large 1 (Dlg1), a PDZ-domain scaffold involved in polarized membrane trafficking. Dlg1 links Kif13B-dependent outward transport and Sec8-dependent exocytosis to MTMR2-mediated PI-turnover (Bolis, Coviello et al. 2009). A role of MTMR2 in neurons was supported by the findings that MTMR2 interacts with NF-L (neurofilament light chain protein) (Previtali, Zerega et al. 2003) and PSD95 (Lee, Kim et al. 2010). The latter contributes to the maintenance of excitatory synapses by regulating endosomal trafficking of AMPA ( $\alpha$-amino-3-hydroxy-5-methyl-4-isoxazolepropionic acid) receptors. Additionally to peripheral neuropathies, MTMR2-deficient mice suffer from defects in spermatogenesis (Mruk and Cheng 2011) due to impaired dynamics of adhesion junctions and defects in membrane trafficking. The different disease phenotypes upon loss of MTMR2 are closely linked to regulation of its catalytic activity and localization by either MTMR5 or MTMR13: While loss of MTMR5 causes spermatogenesis defects, loss of MTMR13 leads to CMT4B2, a disease very similar to CMT4B1.

Unique among the myotubularin family are MTMR3 and MTMR4, which contain a long C-terminal extension bearing an additional FYVE-domain, and form MTMR3-MTMR4 heterodimers (Lorenzo, Urbe et al. 2006). Via its PI(3)P-specific FYVE domain MTMR4 localizes to Rab5-positve early and Rab11-positive recycling endosomes. MTMR4-dependent $\mathrm{PI}(3) \mathrm{P}$ depletion at early endosomes is thought to regulate endosomal sorting at the interface between early and recycling endosomes. While recycling remains unperturbed upon loss of MTMR4, over-expressed MTMR4 impairs TfR recycling and EGFR degradation via a mechanism that requires its phosphatase activity. Also, MTMR4 colocalizes with the exocyst subunit Sec15 and affects localization of VAMP3-containing endosomes (Lorenzo, Urbe et al. 
2006; Naughtin, Sheffield et al. 2010). Additionally to PI(3)P, MTMR4 dephosphorylates RSmads at early endosomes and thereby negatively regulates TGF- $\beta$ (transforming growth factor) (Yu, Pan et al. 2010) and BMP (bone morphogenetic protein) signaling pathways (Yu, He et al. 2013).

\subsection{Endosomal PI(4)P is synthesized by type II PI 4-kinases}

In mammals PI(4)P can be synthesized by the type III (PI4K3 $\alpha$ and PI4K3 $\beta$ ) and type II (PI4K2 $\alpha$ and PI4K2 $\beta$ ) PI 4-kinases (Balla and Balla 2006). Of these only the type II enzymes localize to endosomes and might regulate an endosomal PI(4)P presumably required for endosomal exocytosis. Thus, the type II enzymes are the focus of this study. In brief, PI4K $3 \alpha$ is mainly localized to the ER/ Golgi interface and implicated in ER-to-Golgi transport (Blumental-Perry, Haney et al. 2006). In addition, PI4K3 $\alpha$ maintains the PM pool of PI(4)P (Balla, Tuymetova et al. 2005) and PI(4,5) $\mathrm{P}_{2}$ (Bojjireddy, Botyanszki et al. 2014). At the Golgi complex, PI(4)P-production by PI4K3 $\beta$ and its association with Rab11 are required for secretory membrane traffic and during cytokinesis (Polevoy, Wei et al. 2009). In addition PI4K3 $\beta$ has been found in the nucleus with so far unknown function.

Depending on their subcellular localization, the type II enzymes can function in transport from the TGN or endosomes. Their localization as well as enzymatic activity is regulated via palmitoylation at a conserved stretch of cysteines within the catalytic domain. Notably, the membrane-bound form is more active when compared to its cytosolic counterpart (Barylko, Mao et al. 2009). Furthermore, palmitoylation of PI4K2 $\alpha$ is regulated by cholesterol content of internal membranes (Lu, Sun et al. 2012). At the TGN, PI4K2 $\alpha$-mediated PI(4)P synthesis recruits AP-1 to the Golgi and is required for AP1-dependent post-Golgi traffic of i.e. VSV-G. While in this study PI4K2 $\beta$ depletion did not affect AP-1 recruitment to the TGN (Wang, Wang et al. 2003), others showed that PI4K2 $\beta$ can directly interact with AP-1 and regulate AP-1 dependent endosomal sorting of the Wnt receptor Frizzled (Fz) (Wieffer, Cibrian Uhalte et al. 2013). At the level of endosomes, PI4K2 $\alpha$ and its lipid product PI(4)P localize AP-3 to endosomes to regulates lysosomal cargo delivery (Craige, Salazar et al. 2008) and early-to-late endosomal trafficking of ubiquitinated EGFR or Fz (Minogue, Waugh et al. 2006; Mossinger, Wieffer et al. 2012). Recently, PI4K2 $\alpha$ was shown to associate with early endosomal VAMP3 or late endosomal VAMP7. Herein, TfR recycling requires VAMP3, PI4K2 $\alpha$ and PI4K2 $\alpha$-mediated PI(4)P synthesis (Jovic, Kean et al. 2014). This data 
further indicates that v-SNAREs such as VAMP3 and VAMP7 might determine endosomal targeting of PI4K2 $\alpha$ by defining subdomains for recycling versus degradative sorting. In neurons, PI(4)P synthesis by PI4K2 $\alpha$ on synaptic vesicles is required for exocytosis, but so far thought to be a precursor for PI(4,5) $\mathrm{P}_{2}$ generation at the PM (Guo, Wenk et al. 2003). In nonneuronal cells, PI4K2 $\alpha$ is implicated in PM PI(4,5) $\mathrm{P}_{2}$-generation upon Wnt-stimulation (Qin, Li et al. 2009), while both, PI4K2 $\alpha$ and PI4K2 $\beta$, were shown to regulate Wnt signaling (Mossinger, Wieffer et al. 2012; Wieffer, Cibrian Uhalte et al. 2013). Despite these crucial functions in endosomal trafficking and signaling, PI4K2 $\alpha$ gene trap mice, lacking the catalytic core domain and thus enzymatic activity, are viable with a late onset neurological disease. However, a compensatory role of the truncated PI4K2 $\alpha$ cannot be excluded and might explain the mild phenotype (Simons, Al-Shawi et al. 2009).

\subsection{Aims of this study}

PIs are spatial and temporal hallmarks of endosomal membrane identity and tightly regulated by PI metabolizing enzymes in endosomal membrane traffic. PI conversion in endocytosis from a $\mathrm{PI}(4) \mathrm{P}$ - and $\mathrm{PI}(4,5) \mathrm{P}_{2}$-enriched $\mathrm{PM}$ towards $\mathrm{PI}(3) \mathrm{P}$-enriched endosomes was clarified in detail (Posor, Eichhorn-Grunig et al. 2015). How exit from PI(3)P-positive endosomes en route to the $\mathrm{PI}(4) \mathrm{P}$-positive $\mathrm{PM}$ in endosomal recycling is coordinated by PI metabolizing enzymes remains poorly characterized. In my diploma thesis, I carried out an siRNA-based screen to identity novel PI kinases and phosphatases in endocytic membrane traffic. However, we were unable to discriminate defective endocytosis from secondary defects such as reduced surface receptor levels that led to endocytic defects. This secondary defect could, i.e. be caused by defective recycling and exocytosis. Given that the PI(3)P 3phosphatase MTM1 was among the hits identified in this screen, my thesis work has aimed to delineate the role of MTM1 in endosomal membrane traffic.

Questions we aimed to address include: Does MTM1 regulate endocytic transport and endosomal sorting? Does MTM1 3-phosphatase activity regulate exit from PI(3)P-positive early endosomes en route to the PM? How is PI conversion in endosomal exocytosis coordinated by PI kinases and phosphatases? What are the PI effector proteins required for PI conversion and subsequent PM fusion? 


\section{Material and methods}

\subsection{Materials}

\subsubsection{Chemicals}

If not mentioned otherwise, general chemicals were purchased from Carl-Roth GmbH, Merck, Sigma-Aldrich and Thermo Fisher Scientific. For reagents used in specific applications, the supplier is stated in the respective methods section.

\subsubsection{Buffers, Media and Solutions}

All buffers and solutions were prepared with ultrapure-filtered water. The $\mathrm{pH}$ was adjusted using $\mathrm{NaOH}$ or $\mathrm{HCl}$ unless otherwise specified. Buffers used for specific protocols are specified in the respective methods section.

Table 2: Buffers and solutions used for molecular biology experiments

\begin{tabular}{ll} 
Molecular biology & \\
\hline 10x TBE & $20 \mathrm{mM}$ EDTA \\
& $890 \mathrm{mM}$ Tris \\
& $890 \mathrm{mM}$ Boric acid \\
\hline 6x DNA loading dye & $0.03 \%$ Bromophenol blue \\
& $0.03 \%$ Xylenecyanol FF \\
& $60 \%$ Glycerol \\
& $60 \mathrm{mM}$ EDTA \\
\hline TE buffer & $10 \mathrm{mM}$ Tris pH 8.0 \\
& $2 \mathrm{mM}$ EDTA \\
\hline Antibiotics (stock solutions) & $50 \mathrm{mg} / \mathrm{ml}$ Ampicillin, sterile filtered $(500 \mathrm{x})$ \\
& $10 \mathrm{mg} / \mathrm{ml}$ Kanamycin, sterile filtered $(200 \mathrm{x})$ \\
\hline LB Medium & $1 \%(\mathrm{w} / \mathrm{v})$ yeast extract \\
& $0.5 \%(\mathrm{w} / \mathrm{v})$ NaCl \\
& $0.5 \%(\mathrm{w} / \mathrm{v})$ Trypton \\
& $\mathrm{pH} 7.4$ \\
\hline 2xYT Medium & $1.0 \%(\mathrm{w} / \mathrm{v})$ yeast extract \\
& $1.6 \%(\mathrm{w} / \mathrm{v})$ Trypton \\
& $0.5 \%(\mathrm{w} / \mathrm{v})$ NaCl \\
& $\mathrm{pH} 7.4$
\end{tabular}


Table 3: Buffers and solutions used for biochemical experiments

\section{Biochemistry}

Lysis buffer

$20 \mathrm{mM}$ Hepes $\mathrm{pH} 7.4$

$100 \mathrm{mM} \mathrm{KCl}$

$2 \mathrm{mM} \mathrm{MgCl} 2$

$1 \%$ Triton $\mathrm{X}-100$

$1 \mathrm{mM}$ PMSF

$0.3 \%$ protease inhibitor cocktail (PIC)

\begin{tabular}{ll}
\hline Homogenisation buffer & Lysis buffer without Triton X-100 \\
\hline 10x TBS & $0.2 \mathrm{M} \mathrm{Tris} \mathrm{pH7.6}$ \\
& $1.4 \mathrm{M} \mathrm{NaCl}$ \\
\hline 6x SDS sample buffer & $15 \% \mathrm{SDS}$ \\
& $0.5 \% \beta$-Mercaptoethanol \\
& $0.25 \%$ Bromophenol blue \\
& $0.325 \mathrm{M}$ Tris pH 6.8 \\
& $0.875 \mathrm{M}$ Saccharose
\end{tabular}

\begin{tabular}{|c|c|}
\hline $4 x$ SDS separating gel buffer & $0.4 \% \mathrm{SDS}$ \\
\hline & 1.5 M Tris $\mathrm{pH} 8.8$ \\
\hline 4x SDS stacking gel buffer & $0.4 \%$ SDS \\
\hline & $0.5 \mathrm{M}$ Tris $\mathrm{pH} 6.8$ \\
\hline 10x SDS running buffer & $246 \mathrm{mM}$ Tris \\
\hline & $10 \% \mathrm{SDS}$ \\
\hline & 1.92 M Glycine \\
\hline Semi-dry blotting buffer & 1x SDS running buffer \\
\hline & $10 \%$ Methanol \\
\hline Wet blotting buffer & $25 \mathrm{mM}$ Tris \\
\hline & 192 mM Glycine \\
\hline & $20 \% \mathrm{MeOH}$ \\
\hline Antibody dilution solution & $2 \%$ bovine serum albumin \\
\hline & $0.03 \% \mathrm{NaN}_{3}$ \\
\hline & $0.1 \%$ Tween-20 \\
\hline & $1 x$ TBS \\
\hline & sterile-filtered \\
\hline Antibody blocking solution & $3 \%$ milk powder in $1 \mathrm{x}$ TBS \\
\hline Ponceau staining & $0.3 \%$ Ponceau S \\
\hline & $1 \%$ acetic acid \\
\hline Ponceau destain & $1 \%$ acetic acid \\
\hline $2 \mathrm{x}$ Bradford reagent & 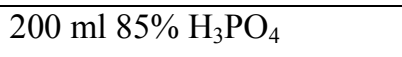 \\
\hline & $100 \mathrm{ml}$ Ethanol \\
\hline & $140 \mathrm{~g} / 1$ Coomassie G250 \\
\hline
\end{tabular}


Table 4: Buffers and solutions used for cell biological experiments

\section{Cell biology}

\begin{tabular}{|c|c|}
\hline 10x PBS & $1.37 \mathrm{M} \mathrm{NaCl}$ \\
\hline & $43 \mathrm{mM} \mathrm{Na}_{2} \mathrm{HPO}_{4}$ \\
\hline & $14 \mathrm{mM} \mathrm{NaH}_{2} \mathrm{PO}_{4}$ \\
\hline & $27 \mathrm{mM} \mathrm{KCl}$ \\
\hline & pH 7.4 \\
\hline $\mathrm{PBS}+\mathrm{MgCl}_{2}$ & $1 x$ PBS \\
\hline & $10 \mathrm{mM} \mathrm{MgCl}_{2}$ \\
\hline PFA fixative & $4 \%$ paraformaldehyde (PFA) \\
\hline & $4 \%$ sucrose \\
\hline & $1 \times \mathrm{PBS}$ \\
\hline & $\mathrm{pH} 7.4$ \\
\hline Goat serum dilution buffer & $10 \%$ goat serum \\
\hline (GSDB) & $100 \mathrm{mM} \mathrm{NaCl}$ \\
\hline & $15.48 \mathrm{mM} \mathrm{Na}_{2} \mathrm{HPO}_{4}$ \\
\hline & $4.52 \mathrm{mM} \mathrm{NaH}_{2} \mathrm{PO}_{4}$ \\
\hline & $0.3 \%$ Triton $\mathrm{X}-100$ \\
\hline
\end{tabular}

\begin{tabular}{|c|c|}
\hline DMEM (Lonza) & Dulbecco's modified eagles medium, $4.5 \mathrm{~g} / 1$ glucose \\
\hline \multirow[t]{4}{*}{ (for HeLa, Hek293) } & Additives: \\
\hline & $10 \%$ fetal calf serum (Gibco) \\
\hline & $100 \mathrm{U} / 1$ penicillin (Gibco) \\
\hline & $0.1 \mathrm{mg} / \mathrm{ml}$ streptomycin (Gibco) \\
\hline \multirow{7}{*}{$\begin{array}{l}\text { DMEM (Lonza) } \\
\text { (for Hek293 FlpIn) }\end{array}$} & Dulbecco's modified eagles medium, $4.5 \mathrm{~g} / 1$ glucose \\
\hline & Additives: \\
\hline & $10 \%$ fetal calf serum (Gibco) \\
\hline & $100 \mathrm{U} / 1$ penicillin (Gibco) \\
\hline & $0.1 \mathrm{mg} / \mathrm{ml}$ streptomycin (Gibco) \\
\hline & $10 \mu \mathrm{g} / \mathrm{ml}$ blasticidin (Invivogen) \\
\hline & $100 \mu \mathrm{g} / \mathrm{ml}$ hygromycin (Hygrogold, Invivogen) \\
\hline \multirow{4}{*}{$\begin{array}{l}\text { DMEM (Lonza) } \\
\text { (for HeLa-M C1) }\end{array}$} & Dulbecco's modified eagles medium, $4.5 \mathrm{~g} / 1$ glucose \\
\hline & Additives: \\
\hline & $10 \%$ fetal calf serum (Gibco) \\
\hline & $1.66 \mu \mathrm{g} / \mathrm{ml}$ puromycin (Invivogen) \\
\hline \multirow{5}{*}{$\begin{array}{l}\text { MEM (Gibco) } \\
\text { (for HDFa, H31, G92-628) }\end{array}$} & Minimum essential medium \\
\hline & Additives: \\
\hline & $15 \%$ fetal calf serum( Gibco) \\
\hline & $100 \mathrm{U} / 1$ penicillin (Gibco) \\
\hline & $0.1 \mathrm{mg} / \mathrm{ml}$ streptomycin (Gibco) \\
\hline
\end{tabular}


Live cell imaging buffer $\quad 1 x$ Hanks balanced salt solution $\left(+\mathrm{Ca}^{2+},+\mathrm{MgCl}_{2}{ }^{2+}\right)$

$10 \mathrm{mM}$ Hepes $\mathrm{pH} 7.4$

$5 \%$ fetal calf serum

Acid wash solution

$100 \mathrm{mM} \mathrm{Na}$ acetate

$100 \mathrm{mM} \mathrm{NaCl}$

pH 5.3

\subsubsection{DNA Oligonucleotides}

Synthetic oligonucleotides used in polymerase chain reactions (PCRs) were purchased from MWG Biotech and BioTeZ, received as lysophilic powder and dissolved in TE buffer to a final concentration of $100 \mu \mathrm{M}$. Working stocks were diluted to $10 \mu \mathrm{M}$ in $\mathrm{ddH}_{2} \mathrm{O}$. All primers used in this study are listed in appendix A.

\subsubsection{Small interference RNA Oligos}

Small interference RNAs (siRNAs) were purchased from MWG Biotech if not mentioned otherwise. All siRNAs used in this study were 21-mers, 23-mers or 27-mers including 3'-dTdT overhangs. For silencing Kif16b and PIKfyve a pool of 4 siRNAs was obtained from Dharmacon (Thermo Fisher Scientific). The scrambled control siRNA used throughout this study corresponds to the scrambled $\gamma 1$ adaptin sequence 5'aaatcggatatcggaatag-3'. siRNAs and Dharmacon SMART pools were received as lysophilic powder and dissolved in RNAse-free siMAX buffer supplied by the manufacturer to a final concentration of $100 \mu \mathrm{M}$.

Table 5: Small interference RNAs used in this study

\begin{tabular}{ll} 
Targeted mRNA & Sequence \\
\hline Exo70 & 5'-ggttaaaggtgactgatta-3' \\
\hline MTM1 & 5'-gatgcaagacccagcgtaa-3' \\
\hline MTMR1 & 5'-gagatagtgtgcaaggata-3' \\
\hline MTMR2 & 5'-ggacatcgatttcaactaa-3' \\
\hline MTMR4 & 5'-cagcataggttacggcaaa-3' \\
\hline MTMR7 & 5'-'tgcaagaactttcagataa-3' \\
\hline PI4K2 $\alpha$ & 5'-ggatcattgctgtcttcaa-3' \\
\hline PI4K2 $\beta$ & 5'-ggttcaagtggaagttact-3' \\
\hline Rab11a & 5'-aagagcgatatcgagctataa-3' \\
\hline Sec3 & 5'-cctgttggatatgggaaacat-3' \\
\hline Sec6 & 5'-ctggaggcagagcatcaacac-3'
\end{tabular}




\begin{tabular}{ll} 
Targeted mRNA & Sequence \\
\hline SNX4 & 5'-tggtcagagtgtcctaaca-3' \\
\hline SNX17 & 5'-ctggettttgaatacctca-3' \\
\hline Vps34 & 5'-cccatgagatgtacttgaacgtaat-3'
\end{tabular}

\subsubsection{Plasmid vectors}

Table 6: Plasmid vector backbones used for cloning purposes and protein expression

\begin{tabular}{|c|c|c|}
\hline Backbone & Properties & Source \\
\hline pEGFP-C & $\begin{array}{l}\text { Used for expression of N-terminal EGFP-tagged fusion proteins in } \\
\text { mammalian cells; EGFP: red-shifted variant of wild-type GFP, that is } \\
\text { optimized for higher expression levels and brighter fluorescence in } \\
\text { mammalian cells; CMV promoter; MCS; SV40 poly A sequence for } \\
\text { proper mRNA processing; Kanamycin resistance cassette that allows } \\
\text { propagation in E.coli and Neomycin/ Kanamycin resistance cassette for } \\
\text { selection of stably transfected eukaryotic cells }\end{array}$ & Clontech \\
\hline pcDNA3.1(+) & $\begin{array}{l}\text { Used for expression of proteins in mammalian cells; CMV promoter; } \\
\text { MCS; BGH poly A sequence for proper mRNA processing; Ampicillin } \\
\text { resistance cassette for propagation in E.coli. }\end{array}$ & $\begin{array}{l}\text { Thermo Fisher } \\
\text { Scientific }\end{array}$ \\
\hline $\begin{array}{l}\text { pc(tag)-Mk and } \\
\text { pcC(tag)-Mk }\end{array}$ & $\begin{array}{l}\text { Based on pcDNA3.1(+) plasmid vector with inserted tag. The tag was } \\
\text { PCR amplified with exogenous } 3 \text { ' KpnI and 5' BamHI restriction sites } \\
\text { used for insertion. Used for expression of N-terminal tagged (pc(tag)- } \\
\text { Mk) or C-terminal tagged (pcC(tag)-Mk) fusion proteins in mammalian } \\
\text { cells. }\end{array}$ & $\begin{array}{l}\text { Modified from } \\
\text { pcDNA3.1 }(+)\end{array}$ \\
\hline pET-28a (+) & $\begin{array}{l}\text { Expression of His-tagged proteins in E.coli; bacteriophage T7 promoter; } \\
\text { IPTG-inducible T } 7 \text { RNA polymerase; Kanamycin resistance cassette that } \\
\text { allows propagation in E.coli }\end{array}$ & Novagen \\
\hline pGEX6P1 & $\begin{array}{l}\text { IPTG-inducible expression of GST-tagged proteins in E.coli; tac } \\
\text { promoter; Ampicillin resistance cassette for propagation in E.coli. }\end{array}$ & $\begin{array}{l}\text { GE Healthcare } \\
\text { Life Sciences }\end{array}$ \\
\hline gpcDNA5/FRT/TO & 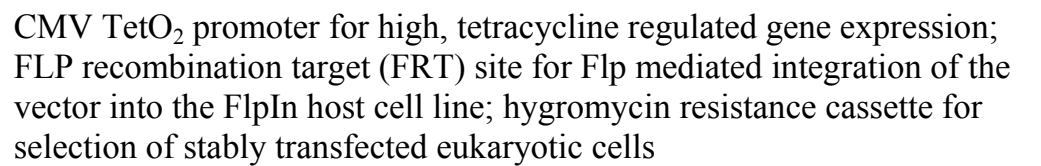 & $\begin{array}{l}\text { Thermo Fisher } \\
\text { Scientific }\end{array}$ \\
\hline
\end{tabular}

Table 7: Plasmid DNA constructs used in this study

RS - restriction sites; mRNA or cds (coding sequence) - NCBI accession number of insert; aa - amino acid sequence encoded by the construct; FL - full length; WT - wild-type; MTM1 siRNA-resistant: created by introducing 4 silent mutations 5'-gatc caggcctagtgtaa-3'

\begin{tabular}{llllll} 
Construct & Backbone & RS & mRNA/ cds & Aa & Comments \\
\hline eGFP-MTM1 & pcEGFP-Mk & BamHI-NotI & AH006018.1 & FL & siRNA-resistant, WT \\
\hline mCherry- & pcmCherry-Mk & BamHI-NotI & AH006018.1 & FL & siRNA-resistant, WT
\end{tabular}

MTM1 


\begin{tabular}{|c|c|c|c|c|c|}
\hline Construct & Backbone & RS & mRNA/ cds & $\overline{\text { Aa }}$ & Comments \\
\hline $\begin{array}{l}\text { eGFP-MTM1 } \\
\text { C375S }\end{array}$ & pcEGFP-Mk & BamHI-NotI & AH006018.1 & FL & $\begin{array}{l}\text { siRNA-resistant, C375S } \\
\text { (catalytically inactive) }\end{array}$ \\
\hline $\begin{array}{l}\text { mCherry- } \\
\text { MTM1 C375S }\end{array}$ & pcmCherry-Mk & BamHI-NotI & AH006018.1 & FL & $\begin{array}{l}\text { siRNA-resistant, C375S } \\
\text { (catalytically inactive) }\end{array}$ \\
\hline $\begin{array}{l}\text { eGFP-MTM1 } \\
\text { P205L }\end{array}$ & pcEGFP-Mk & BamHI-NotI & AH006018.1 & FL & $\begin{array}{l}\text { siRNA-resistant, P205L } \\
\text { (XLCNM mutant) }\end{array}$ \\
\hline $\begin{array}{l}\text { eGFP-MTM1 } \\
\text { R241L }\end{array}$ & pcEGFP-Mk & BamHI-NotI & AH006018.1 & FL & $\begin{array}{l}\text { siRNA-resistant, } \\
\text { R241L } \\
\text { (XLCNM mutant) }\end{array}$ \\
\hline $\begin{array}{l}\text { eGFP-MTM1 } \\
\text { Y397C }\end{array}$ & pcEGFP-Mk & BamHI-NotI & AH006018.1 & FL & $\begin{array}{l}\text { siRNA-resistant, } \\
\text { Y397C (XLCNM } \\
\text { mutant) }\end{array}$ \\
\hline $\begin{array}{l}\text { His-MTM1 } \\
\text { aa } 1-533\end{array}$ & pET-28a (+) & NheI-Not I & AH006018.1 & $1-533$ & $\begin{array}{l}\text { Fragment used to } \\
\text { affinity-purify rabbit } \\
\text { antibody to MTM1 } \\
\text { (rabbit \#330) }\end{array}$ \\
\hline eGFP-MTMR1 & pcEGFP-Mk & BamHI-XhoI & NM_016985.2 & FL & WT, mouse \\
\hline eGFP-MTMR2 & pcEGFP-Mk & $N o t \mathrm{I}-X b a \mathrm{I}$ & NM_016156.5 & FL & WT \\
\hline eGFP-MTMR4 & pEGFP-C & Unknown & NM_004687.4 & FL & $\begin{array}{l}\text { WT, Gift from M. } \\
\text { Clague }\end{array}$ \\
\hline eGFP-MTMR7 & pcEGFP-Mk & BamHI-NotI & NM_004686.4 & FL & WT \\
\hline HA-PI4K2 $\alpha$ & pcDNA5/FRT/TO & EcoRI-NotI & NM_145501.2 & $\mathrm{FL}$ & WT, mouse \\
\hline eGFP-PI4K2 $\alpha$ & pcEGFP-Mk & EcoRI-NotI & NM_145501.2 & FL & WT, mouse \\
\hline $\begin{array}{l}\text { eGFP- } \\
\text { PI4K2 } \alpha \text { D308A }\end{array}$ & pcEGFP-Mk & EcoRI-NotI & NM_145501.2 & FL & $\begin{array}{l}\text { D308A (catalytically } \\
\text { inactive), mouse }\end{array}$ \\
\hline eGFP-PI4K2 $\beta$ & pcEGFP-Mk & EcoRI-NotI & NM_018323.3 & FL & WT \\
\hline eGFP-SNX1 & pcEGFP-Mk & EcoRI-Not $\mathrm{I}$ & AF065483.1 & FL & WT \\
\hline eGFP-SNX3 & pcEGFP-Mk & EcoRI-NotI & AF034546.1 & FL & WT \\
\hline eGFP-SNX4 & pcEGFP-Mk & EcoRV-NotI & AF065485.1 & FL & WT \\
\hline eGFP-SNX8 & pcEGFP-Mk & EcoRV-NotI & AF121858.1 & FL & WT \\
\hline eGFP-SNX15 & pcEGFP-Mk & EcoRI-Not $\mathrm{I}$ & AF175267.2 & FL & $\begin{array}{l}\text { WT } \\
\end{array}$ \\
\hline eGFP-SNX17 & pcCEGFP-Mk & EcoRI-Not $\mathrm{I}$ & NM_014748.3 & FL & WT \\
\hline eGFP-SNX27 & pcEGFP-Mk & EcoRV-NotI & AY044866.1 & FL & WT \\
\hline eGFP-Rab4A & pEGFP-C & Unknown & AF498934.1 & FL & $\begin{array}{l}\text { WT, Gift from P. Van } \\
\text { der Sluis }\end{array}$ \\
\hline eGFP-Rab5A & pcEGFP-Mk & EcoRI-NotI & AF498936.1 & FL & WT \\
\hline $\begin{array}{l}\text { HA-Rab5A } \\
\text { Q79L }\end{array}$ & pcHA-Mk & EcoRI-NotI & AF498936.1 & FL & $\begin{array}{l}\text { Q79L (constitutively } \\
\text { active) }\end{array}$ \\
\hline
\end{tabular}




\begin{tabular}{llllll}
\hline Construct & Backbone & RS & mRNA/cds & Aa & Comments \\
\hline Rab5A Q79L & pcDNA3.1 $(+)$ & EcoRI-NotI & AF498936.1 & FL & $\begin{array}{l}\text { Q79L (constitutively } \\
\text { active) }\end{array}$ \\
\hline eGFP-Rab7 & pEGFP-C & Unknown & Unknown & FL & $\begin{array}{l}\text { WT, Gift from P. Van } \\
\text { der Sluis }\end{array}$ \\
& & & & & WT \\
\hline eGFP-Rab8A & pcEGFP-Mk & EcoRI-NotI & NM_005370.4 & FL & WT, Gift from P. Van \\
\hline eGFP-Rab11A & pcEGFP-Mk & KpnI-BamHI & AF000231.1 & FL & der Sluis
\end{tabular}

\begin{tabular}{llllll}
\hline eGFP-Rab14 & pEGFP-C & Xhol-BamHI & AAF00150 & FL & $\begin{array}{l}\text { WT, Gift from T. } \\
\text { Cezanne }\end{array}$ \\
& & & & & \\
\hline eGFP-Rab35 & pcEGFP-Mk & EcoRI-XbaI & AB232631.1 & FL & WT, mouse \\
\hline eGFP-2x & pcEGFP-Mk & Complex & U43895 & FYVE & Tandem FYVE domain \\
FYVE $_{\text {Hrs }}$ & & & & domain & of Hrs \\
\hline GST-Phox $_{\text {p40 }}$ & pGEX6P1 & NotI-BamHI & AB025219.1 & Phox & Phox domain of p40, \\
& & & & domain & Gift from I. Ganley \\
& & & & $(1-148)$ &
\end{tabular}

\subsubsection{Molecular weight standards}

Table 8: Molecular weight standards

\begin{tabular}{lll} 
Marker & Marker bands & Source \\
\hline \hline Generuler 1kbp DNA ladder & kbp marker band: & Fermentas \\
& $\begin{array}{l}10,8,6,5,4,3.5,3,2.5,2,1.5,1,0.75,0.5,0.25 \\
\text { Prestained protein standard } \\
\end{array}$ & \\
& kDa marker bands: & New England Biolabs \\
& $175,80,58,46,30,25,17,7$ &
\end{tabular}

\subsubsection{Antibodies}

All primary antibodies used in this study are listed in Table 8. Fluorescently-labelled secondary antibodies, chemically conjugated to an AlexaFluor dye were purchased from Thermo Fisher Scientific and used at a dilution of 1:200. HRP-coupled secondary antibodies were obtained from Dianova and used at a dilution of 1:5,000.

Table 9: Primary antibodies used in this study

\begin{tabular}{llllll} 
Antigen & Host & Clone & Source & Dilution & $\begin{array}{l}\text { Dilution } \\
\text { IF/FACS }\end{array}$ \\
& & & & WB & $1: 10,000$ \\
\hline Actin & Mouse & ac-15 & Sigma-Aldrich & - & -
\end{tabular}




\begin{tabular}{|c|c|c|c|c|c|}
\hline Antigen & Host & Clone & Source & $\begin{array}{l}\text { Dilution } \\
\text { IF/FACS }\end{array}$ & $\begin{array}{l}\text { Dilution } \\
\text { WB }\end{array}$ \\
\hline AP-2 $\alpha(\alpha$-adaptin $)$ & Mouse & AP-6 & Hybridoma cell line & $1: 100$ & - \\
\hline APPL1 & Rabbit & & Cell Signaling & $1: 100$ & - \\
\hline$\beta 1$-integrin & Mouse & LM534 & Millipore & $1: 375$ & - \\
\hline$\beta$-tubulin & Mouse & B5-1-2 & Sigma-Aldrich & - & $1: 500$ \\
\hline Clathrin heavy chain & Rabbit & & Abcam & $1: 500$ & - \\
\hline EEA1 & Rabbit & & Cell Signaling & $1: 100$ & - \\
\hline EGFR & Mouse & R-1 & Santa Cruz & $1: 100$ & \\
\hline Exo70 & Mouse & & Millipore & $1: 100$ & $1: 500$ \\
\hline Gadkin & Rabbit & & $\begin{array}{l}\text { Reference } \\
\text { (Schmidt, 2009, PNAS) }\end{array}$ & - & $1: 1,000$ \\
\hline GM130 & Mouse & & BD Transduction & $1: 100$ & - \\
\hline GFP & Rabbit & & Abcam & $1: 500$ & $1: 10,000$ \\
\hline HA & Mouse & HA.11 & Babco & $1: 400$ & - \\
\hline HA-Alexa488 & Mouse & HA.11 & Babco & $1: 100$ & - \\
\hline HA & Rabbit & & Cayman Chemical & $1: 100$ & - \\
\hline $\mathrm{HA}$ & Rabbit & $\mathrm{Y}-11$ & Santa Cruz & - & $1: 500$ \\
\hline LAMP1 & Mouse & & BD Pharmingen & $1: 200$ & - \\
\hline LC3B & Mouse & $4 \mathrm{E} 12$ & MBL International & $1: 100$ & \\
\hline MTM1 & Rabbit & & $\begin{array}{l}\text { Raised against aa 19-33 and aa } \\
\text { 502-516 of human MTM1 }\end{array}$ & - & $1: 250$ \\
\hline $\mathrm{PI}(4) \mathrm{P}$ & Mouse & $\begin{array}{l}\text { Catalog: } \\
\text { Z-P004 }\end{array}$ & Echelon Biosciences & $1: 63$ & - \\
\hline $\mathrm{PI}(4,5) \mathrm{P}_{2}$ & Mouse & $\begin{array}{l}\text { Catalog: } \\
\text { Z-A045 }\end{array}$ & Echelon Biosciences & $1: 200$ & - \\
\hline $\mathrm{PI}(3,4) \mathrm{P}_{2}$ & Mouse & $\begin{array}{l}\text { Catalog: } \\
\text { Z-P034b }\end{array}$ & Echelon Biosciences & $1: 150$ & - \\
\hline $\mathrm{PI} 4 \mathrm{~K} 2 \alpha$ & Rabbit & & $\begin{array}{l}\text { Reference } \\
\text { (Mössinger 2012) }\end{array}$ & - & $1: 2,000$ \\
\hline PIKfyve & Sheep & & Tocris Bioscience & - & $1: 1,000$ \\
\hline Rab5 & Mouse & & BD Transduction & $1: 100$ & - \\
\hline Rab7 & Rabbit & D95F2 & Cell Signaling & $1: 50$ & - \\
\hline Rab11a & Rabbit & & Life Technologies & - & $1: 500$ \\
\hline Sec3 & Rabbit & & Proteintech Group & - & $1: 500$ \\
\hline Sec6 & Mouse & & Stressgen & - & $1: 500$ \\
\hline $\operatorname{Sec} 8$ & Mouse & & BD Transduction & - & $1: 500$ \\
\hline SNX4 & Mouse & & Sigma-Aldrich & - & $1: 500$ \\
\hline SNX17 & mouse & & Proteintech Group & - & $1: 1,000$ \\
\hline TGN46 & Sheep & & AbD Serotec & $1: 200$ & - \\
\hline
\end{tabular}




\begin{tabular}{llllll}
\hline Antigen & Host & Clone & Source & $\begin{array}{l}\text { Dilution } \\
\text { IF/FACS }\end{array}$ & $\begin{array}{l}\text { Dilution } \\
\text { WB }\end{array}$ \\
\hline TfR & Mouse & H68.4 & LifeTechnologies & $1: 200$ & - \\
\hline TfR & Rabbit & & Sigma-Aldrich & $1: 100$ & - \\
\hline Vps26 & Rabbit & & Abcam & $1: 100$ & - \\
\hline Vps34 & Rabbit & D9A5 & Cell Signaling & - & $1: 1,000$
\end{tabular}

\subsubsection{Bacterial strains}

For cloning of recombinant proteins, amplification and storage of plasmid constructs the chemically competent E.coli strain TOP10 (Life Technologies) was used. For expression of recombinant proteins the E.coli strain BL21-CodonPlus (Stratagene) was used. This strain was engineered to contain extra copies of genes that encode rare tRNAs that frequently limit efficient production of heterologous proteins in E.coli.

\subsubsection{Eukaryotic cell lines}

For all cell biological and cell fractionation experiments, unless otherwise specified, HeLa cells were used as a model system for mammalian cells as they are easily applicable for genetic manipulations. HeLa cells are epithelial cervical cancer cells that were derived from a cervical cancer in 1951, donated by Henrietta Lacks. They are characterized by numerous genomic alterations, especially an increased number of chromosomes and genomic integration of the human papilloma virus (HPV80) at multiple sites. HeLa cells were from ATCC and not used beyond passage 30 from original derivation from ATCC.

To study consecutive secretion, a HeLa-M Clone 1 (C1) cell line stably expressing an eGFP-tagged mutant FKBP protein (F36M) was used (Gordon, Bond et al. 2010). This mutant FKBP protein forms ligand-reversible dimers, aggregates in absence of the ligand and cannot be secreted from the ER. Upon ligand (D/D solubilizer, Clontech) incubation these aggregates are resolved and the protein is synchronously secreted from the ER. C1 cells were not used beyond passage 10 .

To study XLCNM in a cell culture model, two XLCNM-patient derived fibroblast cell lines were used. The H31 cell line has a genomic deletion of the entire MTM1 (Laporte, Biancalana et al. 2000; Laporte, Kress et al. 2001) and the G92-628 cell line, referred to as XLCNM patient \#2, has a stop mutation in MTM1 at amino acid 37 (Laporte, Biancalana et al. 2000; Laporte, Kress et al. 2001). HDFa cells (human dermal fibroblast from adult healthy individuals) were obtained from Life Technologies and used as a control cell line. As H31, G92-628 and HDFa are primary cells they were not used beyond passage 12 . 
For immunoprecipitation experiments, the human embryonic kidney cell line Hek293 stably expressing HA-tagged PI4K2 $\alpha$ was generated using the FlpIn ${ }^{\circledR}$ system developed by Life Technologies according to the manufacturer's protocol. Addition of doxycyclin (Invivogen) to the growth medium 24 hours prior to the experiment initiates HA-PI4K2 $\alpha$ expression and assures high protein expression levels especially in concentrated protein lysates as required for biochemical assays.

\subsubsection{Enzymes}

Dream Taq DNA Polymerase and T4 DNA Ligase were purchased from Thermo Fisher Scientific. Vent DNA Polymerase, Phusion DNA Polymerase, restriction enzymes and calf intestinal alkaline phosphatase (CIP) were purchased from New England Biolabs (NEB) and used according to manufacturer's instruction.

\subsubsection{Molecular biology kits}

Large scale and small scale DNA purification kits for isolation of plasmid DNA from E.coli overnight cultures and kits for purification of DNA from agarose gels and PCR reactions were purchased from Macherey-Nagel.

\subsubsection{Supplier}

Abcam

AbD Serotec

Amersham

Andor Technology

ATCC

Babco

Bandelin

BD Biosciences

BD Transduction

BD Pharmingen

Beckman-Coulter

Biometra

BioTez

Biotium
Cambridge, USA

Kidlington, United Kingdom

see GE healthcare

Belfast, United Kingdom

Manassas, USA

Covance, Princeton USA

Berlin, Germany

Mountain View, USA

see BD Biosciences

see BD Biosciences

Krefeld, Germany

Göttingen, Germany

Berlin, Germany

Hayward, USA 
Carl Roth GmbH

Cayman

Cell Signaling

Clontech

Dianova

Enzo Life Sciences

Eurogentec

Eppendorf

Echelon

Fermentas

Fitzgerald

GE healthcare

Gibco

Hamamatsu

Hartmann Analytic

Hidex

Invivogen

Intelligent Imaging (3i)

Licor

Life Technologies

Lonza

Macherey-Nagel

MBL International

Merck

Millipore

MWG-Biotech

New England Biolabs

Nikon

Novagen

Peqlab

Perkin Elmer

Peprotech

Prior Scientific

Promega
Karlsruhe, Germany

Ann Arbor, USA

Danvers, USA

see BD Biosciences

Hamburg, Germany

Lörrach, Germany

S.A. Liège, Belgium

Hamburg, Germany

Salt Lake City, USA

St. Leon-Rot, Germany

Acton, USA

Munich, Germany

see Life Technologies

Hamamatsu, Japan

Brunswick, Germany

Turku, Finland

San Diego, USA

Denver, USA

Lincoln, USA

see Thermo Fisher Scientific

Cologne, Germany

Düren, Germany

Woburn, USA

Darmstadt, Germany

see Merck

Martinsried, Germany

Frankfurt am Main, Germany

Tokyo, Japan

see Merck

VWR International GmbH, Erlangen, Germany

Waltham, USA

New Jersey, USA

Rockland, USA

Muenster, Germany 
Proteintech Group

Roche Diagnostics

Roper Scientific

Santa Cruz Biotech

Sigma-Aldrich

Stratagene

Stressgen

Syngene

Thermo Electron

Thermo Fisher Scientific

Tocris Bioscience

Zeiss
Chicago, USA

Mannheim, Germany

Princeton Instruments, Martinsried, Germany

Santa Cruz, USA

Munich, Germany

La Jolla, USA

see Enzo Life Sciences

Haryana, India

see Thermo Fisher Scientific

Waltham, USA

Bristol, United Kingdom

Jena, Germany

\subsection{Molecular biology methods}

\subsubsection{Plasmid construction}

Cloning strategies were designed using the Vector NTI 10 software (Thermo Fisher Scientific). Primers were designed so their salt-adjusted annealing temperature lies between $50^{\circ} \mathrm{C}$ and $65^{\circ} \mathrm{C}$, using an online tool from the Northwestern University for calculations (web link: http://www.basic.northwestern.edu/biotools/oligocalc.html). G or C bases at the 3' and 5 ' end of the primer were preferred to increase primer specificity and annealing efficiency due to the stronger bonding of $\mathrm{G}$ and $\mathrm{C}$ bases compared to A or T. Using the Vector NTI 10 software plasmids were controlled for frame shifts, compatibility of selected restriction sites or intervening stop codons prior to performing PCR reactions.

\subsubsection{Preparation of complementary DNA (cDNA) libraries}

For cloning of various protein coding sequences, a human cDNA library from HeLa cells was used as template DNA. Therefore, total RNA was isolated from freshly harvested HeLa cells using the SV total RNA isolation system (Promega) and cDNA was reversely transcribed from messenger RNAs (mRNAs) using the Superscript III Reverse Transcriptase (RT, Thermo Fisher Scientific).

Since the efficiency of RNA isolation critically depends on the absence of RNAse, sterile RNAse-free material and cloves were used throughout the preparation. A $10 \mathrm{~cm}$ dish of 
confluent HeLa cells was washed once with PBS, harvested by trypsinisation at $37^{\circ} \mathrm{C}$ for 5 min and cells were resuspended in PBS to a total volume of $10 \mathrm{ml}$ and counted. After sedimentation of cells at $300 \mathrm{xg}$ for $5 \mathrm{~min}$ at room temperature, the pellet was resuspended in 2 $\mathrm{ml}$ ice-cold PBS and split up so a maximum of $1.5 \times 10^{6}$ cells was used per RT reaction. Cells were resuspended in $175 \mu \mathrm{l}$ RNA lysis buffer (RLA) and $350 \mu 1$ RNA dilution buffer, mixed by inversion and incubated for exactly $3 \mathrm{~min}$ at $70^{\circ} \mathrm{C}$ in a thermo cycler. The RLA contains denaturants such as guanidine or urea to disrupt the cells and at the same time inactivate released RNAse from these cells. Detergents might be included if the lipid content of the sample material is especially high. Cell debris was removed by centrifugation at $14,000 \times \mathrm{xg}$ for $10 \mathrm{~min}$ at room temperature. The supernatant was transferred to a new tube and $200 \mu 195 \%$ ethanol was added to precipitate the RNA. The solution was transferred onto a silica membrane spin column, washed once with RNA wash solution and incubated for 15 min with $50 \mu 1$ freshly prepared yellow core buffer containing $10 \mu$ 1 Dnase I (to remove genomic DNA contaminations) and $10 \mu 10.09 \mathrm{M} \mathrm{MnCl}_{2}$. Addition after manganese as a DNAse cofactor favors the digestion of DNA into blunt-ended double-stranded fragments that are unable to religate. The reaction was stopped by adding $200 \mu$ l Dnase stop buffer, washed with RNA washing solution and eluted in nuclease-free water and RNA concentration determined (see section 2.2.11).

Isolated total RNA was immediately used for RT-PCR. Reverse transcription of the total mRNA pool results in a pool or library of cDNA strands that can be subsequently used as template DNA in PCR reactions. $11 \mu 1$ of the RNA preparation, $1 \mu 1$ of dNTPs (Superscript III RT kit) and $1 \mu \mathrm{l}$ of polyT primer (Roche Diagnostics, at $80 \mu \mathrm{M}$ ) were mixed and incubated at $65^{\circ} \mathrm{C}$ for $5 \mathrm{~min}$ to denature the RNA double strands and chilled for $1 \mathrm{~min}$ on ice. After adding $4 \mu \mathrm{l}$ RT-buffer, $1 \mu 10.1$ M DTT, $1 \mu$ l Superscript III RT (all included in the Superscript III RT kit) and $1 \mu$ l RiboLock Rnase Inhibitor (Fermentas) RT was carried out for $5 \mathrm{~min}$ at room temperature, followed by $45 \mathrm{~min}$ at $50^{\circ} \mathrm{C}$. cDNA libraries were stored at $-20^{\circ} \mathrm{C}$.

\subsubsection{Polymerase chain reaction ( $P C R)$}

Polymerase chain reaction is commonly used to selectively amplify a specific target DNA. Thermal cycling between melting, annealing and elongation is used to repeatedly amplify the target DNA. Melting of double stranded DNA at high temperatures assures proper segregation of the double stranded DNA, followed by cooling of the reaction to allow annealing of the single stranded DNA with primers complementary to the 3' and 5' end of the target DNA to provide specificity for a certain DNA target. Finally, double stranded DNA is 
synthesized by a heat-stable DNA polymerase at a higher temperature optimal for enzymatic activity. In the following cycles newly synthesized DNA can additionally be used as template thus leading to exponential amplification of DNA. For cloning purposes the high fidelity Phusion DNA polymerase or the Vent DNA polymerase (New England Biolabs) were used, which have a very low error rate, whereas for Colony PCR a cheaper and more error prone enzyme, Dream Taq polymerase (Fermentas) was used. Polymerase chain reaction was in general used (i) to amplify protein-coding sequences from cDNA or plasmid DNA for subcloning of purified PCR products into chosen expression vectors or (ii) to screen bacterial clones for transformation of DNA plasmids that have integrated the purified PCR products into the vector backbone (colony PCR).

For cloning purposes PCR reactions contained 1x polymerase reaction buffer supplied by the manufacturer, $10 \mu \mathrm{M}$ of each primer, $200 \mu \mathrm{M}$ of each dNTP, 1 unit of Phusion or 2 units of Vent polymerase and $50 \mathrm{ng}$ template DNA or $1 \mu \mathrm{l}$ of cDNA in a final volume of 50 $\mu$ l. For colony PCRs PCR reactions contained 1x Dream Tag buffer, $10 \mu \mathrm{M}$ of each primer, $200 \mu \mathrm{M}$ of each dNTP and 0.625 units of Dream Taq DNA polymerase in a final volume of $25 \mu$ l. For each colony PCR, suitable primers were chosen to amplify the integrated DNA. After preparation of the PCR reaction mix, bacterial colonies were picked from LB-agar plates and stirred in the reaction mix. For amplification of DNA a T3 thermocycler was used and PCR was performed as depicted below. The annealing temperature was chosen according to the salt-adjusted melting temperature for the individual primer pairs and set to a $2-3^{\circ} \mathrm{C}$ higher temperature to avoid primer dimerisation.

\section{Phusion/ Dream Tag Vent}

$\begin{array}{lllll}\text { 1) Initial melting } & 95^{\circ} \mathrm{C} & 45 \mathrm{sec} & 95^{\circ} \mathrm{C} & 2 \mathrm{~min} \\ \text { 2) Melting } & 95^{\circ} \mathrm{C} & 7 \mathrm{sec} & 95^{\circ} \mathrm{C} & 30 \mathrm{sec} \\ \text { 3) Primer annealing } & 50-65^{\circ} \mathrm{C} & 20 \mathrm{sec} & 50-65^{\circ} \mathrm{C} & 30 \mathrm{sec} \\ \text { 4) Elongation } & & & & \\ \quad \text { Phusion, Vent } & 72^{\circ} \mathrm{C} & 15 \mathrm{sec} / \mathrm{kbp} & 72^{\circ} \mathrm{C} & 1 \mathrm{~min} / \mathrm{kbp} \\ \quad \text { Dream Tag } & 72^{\circ} \mathrm{C} & 1 \mathrm{~min} / \mathrm{kbp} & & \\ \text { 5) Final elongation } & 72^{\circ} \mathrm{C} & 10 \mathrm{~min} & 72^{\circ} \mathrm{C} & 10 \mathrm{~min} \\ \text { 6) Storage } & 8^{\circ} \mathrm{C} & \text { infinite } & 8{ }^{\circ} \mathrm{C} & \text { infinite }\end{array}$

Steps 2-4 were repeated in 25 cycles. 
For site directed mutagenesis, a two step PCR amplification approach was used. First, overlapping forward and reverse primer annealing to the template region containing the designed mutations and a non-mutagenic forward and reverse primer annealing at the 3' and 5' end of the cDNA, respectively, were designed and used to amplify 3 ' and 5' fragments containing the mutated nucleotides. The mutagenic primers were chosen so that the overlapping region was at least $15 \mathrm{bp}$ in length and had a salt-adjusted melting temperature of at least $60^{\circ} \mathrm{C}$. To generate a PCR-fusion product, the 5' and 3' PCR fragments were used in equimolar ratios together with the non-mutagenic forward and reverse primer annealing at the start and end of the template DNA. For the initial 5 cycles of this fusion PCR, the primer annealing temperature was set to $8^{\circ} \mathrm{C}$ below the salt-adjusted melting temperature of the overlapping region to increase the probability of generating the fusion product, and subsequently raised by $6^{\circ} \mathrm{C}$ for the following 20 cycles to increase specificity of the PCR reaction. Step 1 and step 2 were carried out at the same day to reduce possible damage of the fusion PCR product.

\subsubsection{Analytical agarose gel electrophoresis}

For analysis and purification of DNA, samples were brought to a final concentration of 1x DNA loading dye (Fermentas). Agarose was dissolved in $1 \mathrm{x}$ TBE buffer to a final concentration of $0.7-1.5 \%(\mathrm{w} / \mathrm{v})$, depending on the size of the DNA fragments. The agarose gel was covered with 1x TBE buffer and run at 90-110 V. DNA was stained with $10 \mathrm{mg} / \mathrm{ml}$ Ethidium bromide for $15 \mathrm{~min}$, visualized with UV light and documented using the G:Box gel documenting system (Syngene).

\subsubsection{Purification of DNA from agarose gels and PCR}

PCR amplified DNA was purified using the NucleoSpin Gel and PCR clean-up kit (Macherey Nagel) and used according to the manufacturer's instructions. For DNA separated by agarose gel electrophoresis the specific band was excised from the agarose gel and DNA was extracted using the NucleoSpin Gel and PCR clean-up kit (Macherey Nagel) according to manufactures instructions.

\subsubsection{Restriction digest}

To properly integrate PCR amplified DNA into a chosen vector backbone, both the PCR product and the vector backbone have to be digested by the same restriction enzymes. 5' of the complementary primer sequence, all primers contained a specific restriction site that is 
later on used for integration of the DNA fragment into the vector backbone followed by a 5' overhang of four randomly chosen nucleotides.

Restriction digest of PCR amplified DNA and preparative digest of plasmid vector DNA was carried out in a final volume of $50 \mu$, containing 9/10 of the PCR reaction or 3 to 5 $\mu \mathrm{g}$ DNA, respectively, 1x BSA (if recommended) and 1x NEB buffer. For digesting PCR products, 15 units of each restriction enzyme were used whereas for preparative restriction digests 3 units/ $\mu$ g DNA were added. Restriction enzymes and buffers were purchased from NEB and used according to the manufacturer's specifications. 1/10 of the PCR reaction was used for analytical agarose gel electrophoresis to control specificity of the PCR. DNA was digested for $1 \mathrm{~h}$ at $37^{\circ} \mathrm{C}$. To prevent re-ligation of linearized vector DNA, the digested vector DNA was dephosphorylated at the 5' ends of the linear DNA double strand by calf intestinal alkaline phosphatase (CIP, NEB), thus decreasing the number of false positive bacterial colonies after transformation of E.coli. Linearized vector DNA was treated with 2 units/ $\mu \mathrm{g}$ DNA for $2 \mathrm{~min}$ at $37^{\circ} \mathrm{C}$. CIP-treated vector DNA and digested PCR products were gel purified (see section 2.2.4 and 2.2.5).

Analytical restriction digests were performed to validate bacterial clones positively identified by colony PCR for integration of purified PCR products into the vector backbone and were performed in a final volume of $20 \mu$, containing $1 \mathrm{x}$ NEB buffer, 1x BSA (if recommended), 3 units of each restriction enzyme and $1 \mu \mathrm{g}$ of DNA. DNA was digested for 1 $\mathrm{h}$ at $37^{\circ} \mathrm{C}$ and analyzed by agarose gel electrophoresis (see section 2.2 .4 ).

\subsubsection{Integration of PCR products into linearized vector backbones}

For ligation of the linearized vector backbone with PCR amplified DNA the molar ratio between the two DNA fragments was measured using a Nanodrop ND-1000 (Peqlab). Ligation was performed in a final volume of $10 \mu \mathrm{l}$, containing 1x ligation buffer (Fermentas), 1 unit of T4 DNA ligase (Fermentas) and a 3:1 molar ration of PCR product : vector backbone. The ligation reaction was carried out over night at $16^{\circ} \mathrm{C}$.

\subsubsection{Preparation of chemically competent E.coli}

To prepare chemically competent E.coli (TOP10 or BL21-CodonPlus strain), single colonies were grown on a LB agar plate overnight, a single colony was picked and inoculated overnight at $37^{\circ} \mathrm{C}$ shaking at $200 \mathrm{rpm}$ in $5 \mathrm{ml} .1 \mathrm{ml}$ of the overnight-culture was used to inoculate a $50 \mathrm{ml}$ LB-medium culture and grown to an $\mathrm{OD}_{600}$ of $0.4\left(2 \times 10^{8}\right.$ cells $\left./ \mathrm{ml}\right)$. Bacteria were harvested by centrifugation at $4,000 \mathrm{rpm}$ at $4{ }^{\circ} \mathrm{C}$ for $10 \mathrm{~min}$ (Thermo Fisher Scientific, 
Heraeus Multifuge X1R), the bacterial pellet was resuspended in $10 \mathrm{ml}$ ice-cold, sterile $0.1 \mathrm{M}$ $\mathrm{CaCl}_{2}$ solution and incubated for $30 \mathrm{~min}$ to $3 \mathrm{~h}$ on ice. The bacteria were sedimented by centrifugation at $4,000 \mathrm{rpm}$ at $4^{\circ} \mathrm{C}$ for $10 \mathrm{~min}$ and resuspended in $2 \mathrm{ml}$ ice-cold, sterile $0.1 \mathrm{M}$ $\mathrm{CaCl}_{2}$ solution. Glycerol was adjusted to a final concentration of $10 \%, 50 \mu \mathrm{l}$ aliquots prepared, snap-frozen in liquid nitrogen and stored at $-80^{\circ} \mathrm{C}$.

\subsubsection{Transformation of chemically competent E.coli}

Chemically competent E.coli (TOP10 or BL21-CodonPlus strain) were transformed with plasmid DNA according to the heat shock protocol. Therefore $7 \mu \mathrm{l}$ ligase reaction was added to $50 \mu \mathrm{l}$ of chemically competent E.coli and incubated for $30 \mathrm{~min}$ on ice, heat shocked for $30 \mathrm{sec}$ at $42^{\circ} \mathrm{C}$ and directly chilled on ice for $5 \mathrm{~min} .500 \mu \mathrm{LB}$-medium were added and the bacterial culture was incubated for $30 \mathrm{~min}$ at $37^{\circ} \mathrm{C}$. After brief centrifugation at maximal speed in a microcentrifuge, bacteria were resuspended in $70 \mu \mathrm{LB}$-medium. Bacterial cultures were plated on agar plates containing the appropriate antibiotic for selection and incubated over night at $37^{\circ} \mathrm{C}$. Bacterial colonies were picked the next day and analyzed using colony PCR (see section 2.2.3) to exclude false-positive bacteria from further analysis. The selected clones were grown overnight at $37^{\circ} \mathrm{C}$ shaking at $200 \mathrm{rpm}$ in $3 \mathrm{ml}$ (mini-prep) or $50 \mathrm{ml}$ (midiprep) LB-medium containing the appropriate antibiotic.

\subsubsection{Isolation of DNA from bacterial cultures}

For isolation of DNA from small volumes of overnight cultures (mini-prep), the NucleoSpin Plasmid kit from Macherey-Nagel was used according to manufacturer's instructions. For larger volumes of overnight cultures (midi-prep) the Nucleobond Ax kit from Macherey-Nagel was used according to manufacturer's instructions. Isolated DNA was controlled for properly inserted PCR products by control digest (see section 2.2.6) before sequencing the constructs.

\subsubsection{Measurement of DNA concentrations}

Nucleic acids specifically absorb UV light at $260 \mathrm{~nm}$ leading to extinction at $260 \mathrm{~nm}$ (E) that is measured using a photometer. DNA concentration can be calculated according to the Lambert- Beer Law $\left(c=E_{\lambda} / d^{*} \varepsilon_{i}\right)$ taking the dilution of the DNA used for each measurement into account. Disposable plastic cuvettes were used with a cross section of $1 \mathrm{~cm}$ (d). The molar extinction coefficient of double stranded DNA is $\varepsilon_{\mathrm{dDNA}}=50 \mathrm{ml} / \mu \mathrm{g}^{*} \mathrm{~cm}$ and 
DNA concentrations can be calculated using the equation depicted below. The ration between the extinction at $260 \mathrm{~nm}$ and the extinction at $280 \mathrm{~nm}$ is used to control the quality of isolated DNA and should lie between 1.8 and 2.1 to exclude large amount of proteins (absorption maximum at $280 \mathrm{~nm}$ ) in the DNA preparation.

\subsubsection{Sequencing}

$1.25 \mu \mathrm{g}$ DNA diluted in $15 \mu \mathrm{l} \mathrm{ddH}_{2} \mathrm{O}$ was sent to MWG Biotech or BioTeZ for sequencing based on the Sanger method (Sanger, Nicklen et al. 1977). Sequencing results were analyzed using Vector NTI 10 software.

\subsection{Cell biological methods}

\subsubsection{Cell culture}

Cells were cultured in a humidified incubator at $37^{\circ} \mathrm{C}$ and $5 \% \mathrm{CO}_{2}$. Cell culture medium used for HeLa, HeLaM Clone 1 (C1), Hek293, Hek293 FlpIn, HDFa, H31 and G92628 cells was listed in Table 1.

Cells were passaged every 2 to 5 days and seeded onto a new cell culture dish in a dilution of 1:2 to 1:10. For the H31 and G92-628 XLCNM-patient cell lines a dilution of 1:2 to 1:4 was used as these patients have a decreased surface pool of adhesion receptors, i.e. integrins, and thereby adhere less efficiently in cell culture. For passaging cells were briefly washed with 1x PBS, trypsinized with $0.05 \%$ trypsin/EDTA (Gibco) for 5 to 7 minutes at $37^{\circ} \mathrm{C}$ and resuspended in an excess of cell culture medium to inactivate the enzymatic activity of trypsin. Primary cells and $\mathrm{C} 1$ cells were not used beyond passage 15 whereas immortalized cell lines were abandoned when reaching passage 35 .

For immunocytochemistry staining of fixed cells $15 \mathrm{~mm}$-coverslips and for live cell imaging 24mm-coverslips were used. Coverslips were coated with $50 \mu 1$ Matrigel (from BD Bioscience, diluted 1:20 in Opti-MEM (Gibco)) for at least 1 hour at $37^{\circ} \mathrm{C}$. Matrigel contains various cell matrix components (entactin, collagen IV, laminin, heparin sulphate proteoglycan) and is used to improve the adherence of cells to otherwise unsuitable surfaces. 


\subsubsection{Plasmid Transfection}

One day prior to transfection, cells were seeded in antibiotics free cell culture medium to reach $80-90 \%$ confluency the next day. For HeLa cells Lipofectamine 2000 (Thermo Fisher Scientific) was used as a transfection reagent according to manufacturer's protocol, using $2 \mu \mathrm{g}$ DNA and $4 \mu \mathrm{l}$ Lipofectamin 2000 for a 6 well. For simultaneous transfection of 2 plasmids $1.5 \mu \mathrm{g}$ of each DNA, for simultaneous transfection of 3 plasmids $1 \mu \mathrm{g}$ of each DNA was used without changing the amount of Lipofectamin 2000. Due to its decreased cytotoxicity and enhanced transfection efficiency, Lipofectamin 3000 was used as a transfection reagent for $\mathrm{HDFa}$ and $\mathrm{H} 31$ cells according to manufacturer's protocol. Expression was allowed overnight and cells analyzed the next day. Lipofectamin 2000 and Lipofectamin 3000 is based on lipofection (Felgner, Gadek et al. 1987), a lipid based transfection method that facilitates the uptake of negatively charged plasmid DNA, complexed with cationic lipids, by fusion of the liposomes with the plasma membrane.

\subsection{3 siRNA Transfection}

For silencing of target genes, cells were seeded on day 0 in antibiotics free growth medium to reach $80 \%$ confluency the next day. HeLa cells were transfected with siRNA using Oligofectamin (Life Technologies) according to manufacturer's protocol, which is a lipofection-based transfection reagent optimized for uptake of small interfering RNAs (siRNAs) into cells. In brief, 200 pmol siRNA and $4 \mu$ l Oligofectamin in a final volume of 1 $\mathrm{ml}$ Opti-MEM were used for a 6 well. After 4 hours the transfection mix was exchanged for antibiotics free growth medium. To achieve optimal knockdown efficiency two rounds of silencing were performed. Cells were transfected on day 1 , expanded on day 2, transfected for a second time on day 3 , seeded for the experiment on day 4 and the experiment was carried out on day 5. For transient overexpression of proteins in knockdown cells plasmids were transfected on day 4 using Lipofectamin 2000 according to manufacturer's protocol.

For knockdown of transiently overexpressed proteins cells were simultaneously transfected with plasmids and siRNA using Lipofectamin 2000 according to manufacturer's protocol, expression was allowed overnight and cells analyzed the next day.

For simultaneous silencing of two proteins, referred to as 'double knockdown', 200 pmol of each siRNA was used, thereby doubling the amount of siRNA, without changing the amount of Oligofectamin. Efficiency of single and double knockdowns was validated by immunoblotting of cell lysates for all siRNA-combinations used in this study. 


\subsubsection{Immunocytochemistry}

Immunocytochemistry stainings are used to visualize endogenous proteins through fluorescent dyes coupled to antibodies directed against the protein of interest in fixed cells. To label a protein or lipid of interest, a primary antibody directed against the appropriate protein/ lipid is recognized by a species specific secondary antibody, which is coupled to a fluorescent dye. Alternatively, expression of fluorescently tagged proteins allows the visualization of a protein in both fixed and live cells.

Cultured cells seeded on Matrigel-coated coverslips were fixed for 10 min with PFA fixative, followed by two washes with PBS. Fixed cells were incubated for 30 minutes at room temperature with GSDB, containing $0.3 \%$ Triton X-100 to permeabilize cellular membranes without disturbing protein-protein interactions and $10 \%$ goat serum to block unspecific antibody binding. $40 \mu 1$ of primary antibodies diluted in GSDB were spotted on parafilm in a humid chamber and coverslips were placed on top. After 1 hour incubation at room temperature, cells were washed three times for 5 minutes with $\mathrm{PBS}+\mathrm{MgCl}_{2}$. Secondary antibodies were diluted 1:200 in GSDB, centrifuged at maximal speed for 10 minutes at room temperature before usage and applied as described above. Cells were incubated with secondary antibody solutions for 1 hour at room temperature, followed by washing them three times for 10 minutes with PBS $+\mathrm{MgCl}_{2}$. Coverslips were dipped twice in $\mathrm{ddH}_{2} \mathrm{O}$ and mounted onto microscopy slides using Immunomount mounting solution (Thermo Electron) containing $1 \mu \mathrm{g} / \mathrm{ml}$ DAPI (4'-6-diamidino-2-phenylindole, Sigma).

For transient overexpression of eGFP-MTM1, cells were washed twice with ice-cold PBS and incubated with PEM (80 mM Pipes pH 6.8, $5 \mathrm{mM}$ EGTA, $1 \mathrm{mM} \mathrm{MgCl}_{2}$ ) containing $0.05 \%$ saponin for $5 \mathrm{~min}$ at $4^{\circ} \mathrm{C}$, thereby selectively decreasing the cytosolic pool of MTM1 while favoring visualization of the membrane associated pool. Saponin permeabilisation is reversible, thus saponin was kept in all solution as indicated. Cells were briefly washed with ice-cold PEM. After fixation with $3 \%$ PFA fixative for 15 min at $4{ }^{\circ} \mathrm{C}$, cells were washed three times with PBS at room temperature, incubated with $50 \mathrm{mM} \mathrm{NH}_{4} \mathrm{Cl}$ for $10 \mathrm{~min}$ and washed again twice with PBS. Immunocytochemistry was done as described above with the exception that Triton X-100 was exchanged for $0.05 \%$ saponin in the blocking solution and cell were washed in PBS $+0.05 \%$ saponin.

For $\mathrm{PI}(3,4) \mathrm{P}_{2-}$ and $\mathrm{PI}(4,5)_{2}$-specific lipid antibody stainings, cells were fixed with $2 \%$ PFA fixative for $20 \mathrm{~min}$ at room temperature and permeabilized using saponin $(0.05 \%$ saponin, $0.1 \% \mathrm{BSA}$ in PBS) for $30 \mathrm{~min}$ at room temperature. Lipid-specific primary antibodies were diluted in 1\% BSA in PBS, incubated for 2 hours, followed by three times 5 
min washes with PBS. Appropriate fluorescently-conjugated secondary antibodies diluted in 1\% BSA in PBS were incubated for 1 hour, followed by three times 10 min washes with PBS.

For PI(3)P- and PI(4)P lipid stainings (Hammond, Schiavo et al. 2009), the purified eGFP-2xFYVE domain of Hrs at a concentration of $0.25 \mu \mathrm{g} / \mathrm{ml}$ or a PI(4)P-specific antibody were used respectively. If indicated PI(3)P was labeled using recombinant GST-tagged Phox domain of p40 chemically conjugated to Alexa Fluor 488 which was a kind gift from I. Ganley. Cells were fixed with $2 \%$ PFA fixative for $15 \mathrm{~min}$ at room temperature, briefly washed three times with $\mathrm{PBS}+50 \mathrm{mM} \mathrm{NH} \mathrm{Nl}_{4} \mathrm{Cl}$ to quench free aldehyde groups and permeabilized with $20 \mu \mathrm{M}$ digitonin diluted in buffer A (20 mM Pipes pH 6.8, $137 \mathrm{mM} \mathrm{NaCl}$, $2.7 \mathrm{mM} \mathrm{KCl}$ ). After washing three time with buffer A, unspecific antibody binding was blocked using $5 \%$ goat serum, $50 \mathrm{mM} \mathrm{NH}_{4} \mathrm{Cl}$ in buffer A, including purified eGFP-2xFYVE domain if applicable, followed by washing cells twice for 5 min with buffer A. Primary antibodies (GFP-specific antibody for eGFP-2xFYVE, PI(4)P-specific antibody or GST-phoxAlexaFluor488 (diluted 1:200)) diluted in buffer A containing 5\% goat serum were incubated for 1 hour, followed by three washes for $5 \mathrm{~min}$ with buffer A. Appropriate, fluorescently labeled secondary antibodies diluted in buffer A containing $5 \%$ goat serum were incubated for $45 \mathrm{~min}$, followed by four washes for 5 min with buffer A, post-fixation with $2 \%$ PFA fixative for $5 \mathrm{~min}$ and three washes for $5 \mathrm{~min}$ with $\mathrm{PBS}+50 \mathrm{mM} \mathrm{NH}_{4} \mathrm{Cl}$. Before mounting, cells were transferred to PBS to avoid quenching of fluorophores by ammonium chloride. For wortmannin (WM, Sigma-Aldrich) treatment cells were incubated with $2 \mu \mathrm{M}$ WM (dissolved in dry DMSO) or DMSO as a control, diluted in serum-free medium, for $30 \mathrm{~min}$ at $37^{\circ} \mathrm{C}$. Subsequent PI(3)P staining was performed as described above.

\subsubsection{Transferrin and EGF uptake assays}

For quantitative uptake of fluorescently-labeled Tf, XLCNM-patient fibroblasts (H31, G92-628), healthy control fibroblast (HDFa) and siRNA-treated HeLa cells were seeded on Matrigel-coated coverslips and starved for 1 hour in serum-free medium. Cells were incubated with $25 \mu \mathrm{g} / \mathrm{ml}$ Tf-Alexa647 (Life Technologies, spun down at 14,000 rpm for 5 minutes using a VWR Micro Star 17R to remove ligand precipitates and diluted in serum-free medium) for 10 min at $37^{\circ} \mathrm{C}$. After being washed twice with ice-cold PBS $+\mathrm{MgCl}_{2}$, cells were acid washed at $\mathrm{pH} 5.3$ for $1 \mathrm{~min}$ on ice to remove surface-bound $\mathrm{Tf}$, followed by two times washing with ice-cold PBS $+\mathrm{MgCl}_{2}$ and fixation with ice-cold PFA fixative for $45 \mathrm{~min}$ at room temperature. 
For Tf uptake followed by immunocytochemistry stainings (see section 2.3.4), HeLa cells treated with siRNAs and plasmid constructs as indicated for the respective experiment were seeded on Matrigel coated coverslips. Cells were starved for 3 hours in serum-free medium and treated with $25 \mu \mathrm{g} / \mathrm{ml}$ Tf-Alexa647, Tf-Alexa568 or Tf-Alexa488 (Life Technologies, spun down at 14,000 rpm for 5 minutes, diluted in serum-free medium) for 30 min at $37^{\circ} \mathrm{C}$ to allow $\mathrm{Tf}$ uptake to reach saturation. Cells were washed twice with PBS + $\mathrm{MgCl}_{2}$, followed by immunocytochemistry staining as described in section 2.3.4.

For TfR surface labeling, XLCNM-patient fibroblasts (H31, G92-628), healthy control fibroblast $(\mathrm{HDFa})$ and siRNA-treated HeLa cells were seeded on Matrigel-coated coverslips and starved for 1 hour in serum-free medium. Cells were incubated with $25 \mu \mathrm{g} / \mathrm{ml} \mathrm{Tf}$ Alexa647 (spun down at $14.000 \mathrm{rpm}$ for 5 minutes, diluted in serum-free medium) for $45 \mathrm{~min}$ at $4^{\circ} \mathrm{C}$ to block endocytosis, washed three times with ice-cold $\mathrm{PBS}+\mathrm{MgCl}_{2}$ and fixed with ice-cold PFA fixative for $45 \mathrm{~min}$ at room temperature.

Tf uptake and surface labeling were analyzed by epifluorescent microscopy as described in section 2.3.11.

Alternatively, quantitative uptake of Transferrin was measured using radioactively labeled ${ }^{125}$ I-Tf (Perkin Elmer). Therefore, siRNA-treated HeLa cells were seeded in a 24-well plate in triplicates and starved for 1 hour in serum-free medium containing $0.1 \%$ BSA and 20 $\mathrm{mM}$ Hepes $\mathrm{pH}$ 7.4. Cells were stimulated with $1 \mu \mathrm{g} / \mathrm{ml}{ }^{125} \mathrm{I}-\mathrm{Tf}$ in starvation medium at $37^{\circ} \mathrm{C}$ for the indicated time points and washed twice on ice with ice-cold PBS. Surface-bound ${ }^{125} \mathrm{I}$ Tf was removed by an acid wash with $0.2 \mathrm{M}$ acetic acid, $0.5 \mathrm{M} \mathrm{NaCl}$ at $\mathrm{pH} 2.5$ for $5 \mathrm{~min}$ on ice, collected and radioactivity was measured using a scintillation counter (HIDEX 300SL). Cells were dried at room temperature for $5 \mathrm{~min}$, lysed with $1 \mathrm{M} \mathrm{NaOH}$ for $60 \mathrm{~min}$ and radioactivity of the lysate was measured (corresponding to internalized Tf). Non-specific binding was measured for each time point in the presence of a 300-fold excess of cold human holo-Tf (Sigma-Aldrich) and subtracted from all values. The ratio of internalized to surfacebound $\mathrm{Tf}$ was plotted over time to visualize the time-course of Tf internalization. Transferrin endocytic rates were calculated from the slopes of these curves $\left(K_{m}\right.$ values) using Prism software (GraphPad).

For EGF (Epidermal Growth Factor) degradation, radioactively labeled ${ }^{125}$ I-EGF (Perkin Elmer) was used. Therefore, siRNA-treated HeLa cells were seeded in a 24-well plate in triplicates and starved for 4 hour in serum-free medium containing $0.1 \%$ BSA and $20 \mathrm{mM}$ Hepes pH 7.4. Cells were stimulated with $25 \mathrm{ng} / \mathrm{ml}{ }^{125} \mathrm{I}$-EGF diluted in starvation medium for $15 \mathrm{~min}$ at $37^{\circ} \mathrm{C}$ and washed twice on ice with ice-cold PBS. Remaining, surface-bound ${ }^{125} \mathrm{I}-$ 
EGF was removed by a mild acid wash with $0.2 \mathrm{M}$ acetic acid, $0.5 \mathrm{M} \mathrm{NaCl}$ at $\mathrm{pH} 4.5$ for 3 min on ice. To allow endosomal progression of internalized EGF-bound receptor, cells were washed twice with pre-warmed starvation medium and incubated with $4 \mu \mathrm{g} / \mathrm{ml}$ cold human EGF (Peprotech) in starvation medium at $37^{\circ} \mathrm{C}$ for the indicated time points. Cells were transferred to ice, the medium was collected and subjected to trichloroacetic acid (TCA; 5\% TCA, $0.5 \% \mathrm{BSA}$ at $4{ }^{\circ} \mathrm{C}$ overnight) precipitation. By this, recycled, intact EGF is separated from degraded EGF released into the medium. After washing cells twice with ice-cold PBS, the remaining, surface-bound ${ }^{125} \mathrm{I}-\mathrm{EGF}$ was removed by an acid wash with $0.2 \mathrm{M}$ acetic acid, $0.5 \mathrm{M} \mathrm{NaCl}$ at $\mathrm{pH} 2.5$ for $2 \mathrm{~min}$ on ice, collected and radioactivity was measured. Cells were dried at room temperature for $30 \mathrm{~min}$, lysed with $1 \mathrm{mM} \mathrm{NaOH}$ for $30 \mathrm{~min}$ and cell lysates were subjected to TCA-precipitation. TCA-precipitated (left-over internal, intact EGF) and TCA-soluble (degraded EGF) fraction were separated by centrifugation at 13,000xg for 5 min at $4^{\circ} \mathrm{C}$. The pellet was dissolved in $1 \mathrm{M} \mathrm{NaOH}$ and the radioactivity in both pellet and supernatant was measured. Non-specific binding was measured for each time-point in the presence of a 300-fold excess of cold human EGF (Peprotech) and was subtracted from all values.

Total internalized EGF was calculated as the sum of all radioactive measurements and set to $100 \%$. Recycled EGF was defined as the sum of radioactivity measured in the TCAprecipitated fraction from the medium supernatant and acid wash, whereas degraded EGF was defined as the sum of radioactivity measured in the TCA-soluble fraction from the medium supernatant, acid wash and cell lysate. The TCA-precipitated fraction of the cell lysate contains the left-over internalized, non-degraded EGF. All EGF-populations were normalized to total internalized EGF and degraded EGF was plotted over time.

\subsubsection{Autophagy assay}

siRNA-treated HeLa cells were seeded on Matrigel-coated coverslips and fresh serumcontaining medium was added $2 \mathrm{~h}$ prior to fixation. In case of Bafilomycin A1 (BafA1 from Streptomyces griseus, Sigma-Aldrich) treatment cells were washed three times with Hanks' balanced salt solution (HBSS, Gibco) and incubated with $100 \mathrm{nM}$ BafA1 (dissolved in dry DMSO) diluted in HBSS for $3 \mathrm{~h}$. Control cells were incubated with DMSO diluted in serumcontaining medium. After fixation with PFA fixative for $30 \mathrm{~min}$, cells were washed three times with PBS and permeabilized with $200 \mu \mathrm{g} / \mathrm{ml}$ Digitonin diluted in PBS for $10 \mathrm{~min}$, followed by three washes in PBS. Cells were incubated with the primary antibody diluted in PBS for $1 \mathrm{~h}$, followed by three washes with PBS. Secondary antibodies diluted in PBS were 
incubated for $1 \mathrm{~h}$, followed by three washes in PBS. The samples were analyzed by epifluorescent microscopy as described in section 2.3.11.

\subsubsection{Cholera toxin uptake}

siRNA-treated HeLa cells seeded on Matrigel-coated coverslips were serum-starved for $30 \mathrm{~min}$ and treated with $1 \mu \mathrm{g} / \mathrm{ml}$ Cholera toxin subunit B (Ctx) CF568 (Biotium) for 45 min at $37^{\circ} \mathrm{C}$, followed by $30 \mathrm{~min}$ chase in starvation medium. After a brief PBS wash, cells were fixed with PFA fixative for 30 min, washed twice with PBS, permeabilized using GSDB for $15 \mathrm{~min}$ and incubated with primary antibody diluted in GSDB for $30 \mathrm{~min}$. After three brief washes with PBS, secondary antibody diluted in GSDB was incubated for $30 \mathrm{~min}$, followed by three brief washes in PBS. The samples were analyzed by epifluorescence microscopy to quantitatively analyze Ctx uptake and by spinning disc confocal microscopy to quantify retrograde trafficking of Ctx to the Golgi complex, labeled by a GM130-specific antibody as described in section 2.3.13.

\subsubsection{Consecutive secretion assay}

siRNA-treated HeLaM C1 cells (Gordon, Bond et al. 2010) seeded on Matrigel-coated coverslips were washed once with PBS and treated with $1 \mu \mathrm{M} \mathrm{D} / \mathrm{D}$ solubilizer diluted in HBSS to initiate secretion of the reporter construct. To halt secretion at the indicated time points, cells were placed on ice, washed twice with ice-cold PBS $+\mathrm{MgCl}_{2}$ and fixed with PFA fixative for $20 \mathrm{~min}$ at room temperature, followed by two times washing with PBS. Secretion of the GFP-tagged reporter construct was analyzed by epifluorescence microscopy as described in section 2.3.11. 15 min after initiating secretion the reporter is localized at the Golgi complex. To calculate the synchronized secretion from the Golgi complex, all time points were normalized to $15 \mathrm{~min}$.

\subsubsection{Elevation of PI(3)P and PI(4)P by PIP/AM treatment}

An elegant way to artificially, non-disruptively elevate cellular levels of distinct phosphoinositide species is the use of PIP/AMs. PIP/AMs are cell-permeable acetoxy methyl ester (AM)-protected phosphatidylinositol derivatives. The free oxygens of the phosphate head group are coupled to AM, while at the free hydoxyl groups of the myo-inositol ring butyrate groups are introduced. To allow cell entry, the negatively charged PIP is masked by the AM-protection group, which is removed by endogenous esterases and lipases within the cytosol partially occurring at membrane interfaces. Thereby the biologically active, negatively 
charged lipid is released and randomly integrates into subcellular membranes (Subramanian, Laketa et al. 2010).

For treatment of cells, PI(3)P/AM and PI(4)P/AM were dissolved in dry DMSO and mixed with an equal volume of 10\% pluronic F127 in DMSO (Sigma-Aldrich). PIP/AMs were diluted in serum-free medium to a final concentration of $100 \mu \mathrm{M}$. For immunocytochemistry stainings, cells seeded on Matrigel-coated coverslips were treated with DMSO + pluronic F127 (control) or PIP/AMs for $30 \mathrm{~min}$ at $37^{\circ} \mathrm{C}$ and then processed as described in section 2.3.4. For TfR immunocytochemistry stainings, cells were incubated with $25 \mu \mathrm{g} / \mathrm{ml}$ Tf-Alexa647 in parallel to the PIP/AM treatment. For live-cell TIRF microscopy cells seeded on Matrigel-coated coverslips were treated with $25 \mu \mathrm{g} / \mathrm{ml}$ Tf-Alexa647 and $\mathrm{DMSO}+$ pluronic F127 (control) or PIP/AMs for $30 \mathrm{~min}$ at $37^{\circ} \mathrm{C}$, directly followed by livecell imaging.

\subsubsection{Inhibition of Vps34 by VPS34-IN1 treatment}

VPS34-IN1 is a recently developed, highly selective and potent inhibitor $(25 \mathrm{nM} \mathrm{IC} 50)$ of the class III PI 3-kinase Vps34 without affecting other protein as well as lipid kinase activities including class I and class II PI 3-kinases (Bago, Malik et al. 2014).

For the analysis of fixed cells siRNA-treated HeLa cells, H31 or HDFa cells were seeded on Matrigel-coated coverslips. To address the dose dependency of VPS34-IN1 treatment on phenotypic changes, i.e. accumulation of $\beta 1$-integrin, XLCNM-patient fibroblasts (H31) and healthy control fibroblasts (HDFa) were treated with DMSO or VPS34IN1 at a concentration of 0.01-1 $\mu \mathrm{M}$ dissolved in dry DMSO and diluted in serum-containing medium for $48 \mathrm{~h}$, adding freshly diluted VPS34-IN1 or DMSO after $24 \mathrm{~h}$. To address the time dependency of inhibitor treatment, MTM1-depleted and scrambled siRNA treated HeLa cells were used due to the milder, more reversible accumulation of $\beta 1$-integrin upon relatively acute genetic manipulation of MTM1 expression. Therefore, cells were treated with DMSO or $1 \mu \mathrm{M}$ VPS34-IN1 diluted in serum-containing medium for the indicated time points. The samples were analyzed by epifluorescence microscopy as described in section 2.3.11.

For live-cell TIRF imaging (see also section 2.3.11) siRNA-treated HeLa cells seeded on Matrigel-coated coverslips were treated with $50 \mu \mathrm{g} / \mathrm{ml}$ Tf-Alexa647 and DMSO or $1 \mu \mathrm{M}$ VPS34-IN1 diluted in serum-containing medium for $60 \mathrm{~min}$ at $37^{\circ} \mathrm{C}$. Live-cell imaging was performed in the presence of DMSO or VPS34-IN1, respectively. 


\subsubsection{Fluorescence microscopy}

Fluorophores can be excited from the ground electronic state to a higher energy level by absorbing photons of a defined wavelength characteristic for each fluorescent dye. Relaxation to the ground electronic state emits light of a defined but longer wavelength.

As MTM1-depleted cells are on average double the size of scrambled siRNA treated controls, all measurements were normalized to cell area, if cell size can be extracted from the data. Fluorescence sum intensity normalized to the cell area is indicated as normalized density. Depending on the experimental context one of the following imaging techniques was chosen.

Epifluorescence microscopy. For quantitative analysis of total fluorescence intensities normalized to the cell area an epifluorescence wide field microscope was used. Fluorescence intensity from various layers are acquired around the chosen focal plane, sampling the whole volume of the cell and thereby useful for quantification of total florescence intensities, i.e. $\mathrm{Tf}$ surface and uptake, LC3B level and EGFR level. The ratio of internalized Tf to surface-bound Tf was used to distinguish between uptake and recycling defects. Due to small differences in TfR levels in HDFa and H31 cells, surface bound Tf was normalized to total TfR levels. TfR levels in HDFa and H31 cells were analyzed by epifluorescence microscopy.

Two different setups were used: (i) Nikon Eclipse Ti microscope (eGFP filter set: F36526; TexasRed filter set: F36-504; Cy5 filter set: F46-009; Dapi filter set: F46-000), equipped with a $\times 40$ oil-immersion objective (Nikon), a sCMOS camera (Neo, Andor), a 200 Watt mercury lamp (Lumen 200, Prior), operated by open-source ImageJ-based micromanager software and quantified using open-source ImageJ software and (ii) Zeiss Axiovert 200M with DG4 excitation unit and Coolsnap HQ2 EM-CCD camera (Roper Scientific) operated and analyzed by Slidebook software (Intelligent Imaging).

Spinning disc confocal microscopy. If subcellular resolution of intracellular structures and z-resolution was required, protein and lipid immunocytochemistry stainings were routinely analyzed and quantified using a spinning disc confocal microscope (Ultraview ERS, Perkin Elmer, connected to a Zeiss Axiovert 200M and equipped with an EE-CCD-camera (Hamamatsu)) operated and analyzed using Volocity imaging software (Improvision, Perkin Elmer). To achieve high image acquisition speed of thin optical sections with minimal photobleaching and high resolution, the spinning disc confocal microscope uses two spinning pinhole discs compared to point-by-point sample scanning of laser scanning microscopes. For all quantifications, protein and lipid stainings were normalized to cell area. 
For quantification of TfR localization, cells with either perinuclear or peripheral TfR localization were counted. Peripheral TfR localization was defined as cells with either TfR dispersion or TfR accumulations at the cell periphery as shown in example images (Results Fig. ) and the normalized fraction of cells with perinuclear TfR localization was quantified. The amount of colocalization between two channels was quantified using thresholded Pearson's Correlation Coefficients. To quantify the amount of colocalization at peripheral sites, thresholded Pearson's Coefficients were calculated in three randomly chosen 100 pixel x 100 pixel squares in the cell periphery. For averaged line scans, line profiles were calculated as the mean fluorescence intensity averaged over 100 pixels. Maximum intensity projections were calculated from z-stacks with $200 \mathrm{~nm}$ spacing between slices covering the whole cell.

Total internal reflection fluorescence (TIRF) microscopy. TIRF microscopy was performed using a Nikon Eclipse Ti microscope, equipped with an incubation chamber $\left(37^{\circ} \mathrm{C}\right)$, a x60 TIRF objective (oil-immersion, Nikon), a sCMOS camera (Neo, Andor), a 200 Watt mercury lamp (Lumen 200, Prior), a triple-color TIRF setup (laser lines: 488nm, 568nm, $647 \mathrm{~nm}$ ) and operated by open-source ImageJ-based micromanager software. To selectively illuminate fluorophores in close proximity to or at the cell surface we applied TIRF microscopy. In TIRF microscopy the specimen is illuminated using a laser beam set to a variable angle in a TIRF objective. When set to the critical angle of total internal reflection, the laser beam is reflected at the interface between two media of different diffraction indices (glass-coverslip and aqueous specimen medium), thereby creating an evanescence field. The intensity of the evanescent field decays exponentially with vertical distance from the interface. Thus, it excites the specimen in 60 to $100 \mathrm{~nm}$ distances from the phase boundary, but the penetration depth can go up to $200 \mathrm{~nm}$ distance, depending on the refraction indices of the two media, the wavelength and entrance angle of the laser beam. In a cellular context the the phase boundary is the plasma membrane (Axelrod, Burghardt et al. 1984).

To analyze and quantitatively measure Tf exocytosis in live-cells, cells seeded on Matrigel-coated coverslips were treated with $50 \mu \mathrm{g} / \mathrm{ml}$ Tf-Alexa647 diluted in serum-free medium for $30 \mathrm{~min}$ at $37^{\circ} \mathrm{C}$ and $5 \% \mathrm{CO}_{2}$. Time-lapse movies spanning $15-30 \mathrm{sec}$ of 5 to 10 cells with a frame rate of $5 \mathrm{~Hz}$ were recorded. For all time-lapse movies, the $488 \mathrm{~nm}$ channel was acquired before the $647 \mathrm{~nm}$ channel unless indicated otherwise. Fusion events with the plasma membrane were defined by their characteristic time course (appearance, broadening/ spreading of the fluorescence signal and disappearance), counted and normalized to cell area. Representative kymographs were chosen over 30 seconds along a line of 400 pixel in length. 


\subsubsection{Flow cytometry}

Compared to immunocytochemistry staining analyzed by microscopy techniques, flow cytometry has the advantage to measure fluorescence intensity by a current-automated, nonslide based approach. Flow cytometry simultaneously measures physical characteristics of single cells, i.e. cell size and granularity, as well as fluorescence intensity by using a laser beam which excites the cells as they flow through the measuring chamber. If the laser beam strikes the cell, light is scattered along the axis of the laser beam (forward scatter, FSc), measuring the intrinsic cell size and reflected of the cell (side scatter, SSc), measuring cell granularity. Viable cells are gated according to FSc and SSc.

On ice, cells were washed once with ice-cold PBS and detached from the culture dish by 5 min incubation on ice with $0.1 \%$ PronaseE (Sigma-Aldrich), $0.5 \mathrm{mM}$ EDTA solution in PBS. Cells were resuspended in PBS, pelleted at $300 \mathrm{xg}$ for $5 \mathrm{~min}$ at $4{ }^{\circ} \mathrm{C}$ (VWR Micro Star 17R) and fixed in PFA fixative for $20 \mathrm{~min}$ at room temperature. 10 times excess volume of blocking solution (0.05\% saponin, $0.01 \%$ BSA in PBS) was added, cells were pelleted and resuspended in blocking solution. After $15 \mathrm{~min}$ primary antibody (mouse- $\alpha$-TfR, see also Table 8) was added and incubated for an additional 1hour. After washing once with blocking solution cells were incubated for 1 hour with secondary antibody diluted in blocking solution, followed by washing once with $0.2 \%$ BSA in PBS. Cells were resuspended in $0.2 \%$ BSA in PBS and analyzed by flow cytometry using a BD LSRFortessa (BD Bioscience).

\subsubsection{Statistical analysis}

For analysis of experiments comprising multiple independent experiments $(n)$, statistically significant estimates for each sample were obtained by choosing an appropriate sample size, correlating to 15 to 30 images per condition for microscopy-based quantifications. No statistical methods were used to predetermine sample size. Cells were chosen arbitrarily according to the fluorescent signal in a separate channel, which was not used for quantification. Experiments were quantified as described in fluorescence microscopy, section 2.3.11. Data are presented as mean of $n$ experiments \pm standard error of the mean (SEM). Unfortunately, with minimally 3 and maximally 5 independent experiments it is not possible to check normal distribution of the mean data points. However, normal distribution within a single experiment was checked on a routinely basis. For cell biological experiments, statistical tests were performed using the two-tailed, unpaired $t$-test, without excluding samples from statistical analysis. This test is used to compare two independent groups when deviations in both directions from a reference value are considered possible. For biochemical 
experiments (see section 2.4.8), statistical tests were performed using the one-sample $t$-test, without excluding samples from statistical analysis. In these experiments, due to technical reasons only a single control, mostly scramble-siRNA treated cells, was chosen and, thus, used for data normalisation. Hence, error bars could not be calculated for the control. In these cases, the one-sample $t$-test, which uses the control as a reference without requiring error bars, was taken for statistical analysis. Data analysis was done using Microsoft Excel or Prism GraphPad. Figures and models were prepared using Adobe Photoshop CS6.

\subsection{Biochemical methods}

\subsubsection{Expression of recombinant proteins in E. coli}

Overnight cultures of BL21-CodonPlus E.coli expressing His $_{6}$-tagged fusion proteins were diluted 1:20 in 500 $\mathrm{ml}$ 2xYT-Medium containing the appropriate antibiotics. The scale was chosen according to the experimental approach. Bacterial growth was allowed at $37^{\circ} \mathrm{C}$ to reach an $\mathrm{OD}_{600}$ of 0.7 to 0.8 . Recombinant protein expression was induced by addition of isopropyl thiogalactoside (IPTG) to a final concentration of $0.5 \mathrm{mM}$ and grown for an additional 4 hours at $30^{\circ} \mathrm{C}$. Bacteria were harvested by centrifugation at 5,000 rpm for $15 \mathrm{~min}$ at $4^{\circ} \mathrm{C}$ (Thermo Fisher Scientific, Sorvall RC 5C, SLA-3000). The supernatant was completely removed and the bacterial pellet frozen at $-20^{\circ} \mathrm{C}$.

\subsubsection{Purification of His ${ }_{6}$-tagged MTM1 fragment expressed in E. coli for subsequent antibody purification}

In order to affinity purify the polyclonal antibody against MTM1 from serum, a MTM1 fragment, spanning amino acids 1 to 533 excluding the C-terminal flexible region of MTM1, fused to a hexahistidin (His 6 )-tag was used. To purify His 6 -tagged recombinant proteins from E.coli, Ni-NTA beads were used, taken that Histidin binds $\mathrm{Ni}^{2+}$ which is linked to beads via the nitrilotriacetic acid (NTA)-chelator.

BL21-bacterial pellets were thawed on ice and resuspended in $25 \mathrm{ml}$ resuspension buffer $(20 \mathrm{mM}$ Tris $\mathrm{pH} 8.0,500 \mathrm{mM} \mathrm{NaCl})$ per 11 E.coli culture. Further $500 \mathrm{U}$ benzonase (DNAse), PMSF to a final concentration of $1 \mathrm{mM}$ and a tip of a spatula of lysozyme (an enzyme degrading the bacterial cell wall, purified from chicken egg white) were added. After 15 min incubation on ice, cells were sonicated once for 2 min on ice (Bandelin, $50 \%$ duty cycles, $70 \%$ power), Chaps was added to $1 \%$ and $\mathrm{NaN}_{3}$ to $0.001 \%$ final concentration. 
Pelleted (His ${ }_{6}$-tagged MTM1 in inclusion bodies) and soluble fraction were separated by sedimentation at $35,000 \mathrm{xg}$ for $15 \mathrm{~min}$ at $4^{\circ} \mathrm{C}$ (Thermo Fisher Scientific, Sorvall Evolution $\mathrm{RC}, \mathrm{SS}-34)$. The supernatant was brought to a final concentration of $0.5 \%$ Chaps and $4 \mathrm{M}$ urea by diluting it $1: 1$ with denaturating buffer $(8 \mathrm{M}$ urea, $20 \mathrm{mM}$ Tris $\mathrm{pH} 8.0,500 \mathrm{mM}$ $\mathrm{NaCl}$ ). After washing the pellet four times by vigorously resuspending the pellet in $20 \mathrm{ml}$ washing buffer (20 mM Tris $\mathrm{pH} 8.0,500 \mathrm{mM} \mathrm{NaCl}, 0.1 \%$ Chaps), followed by sedimentation at $9,000 \mathrm{xg}$ for $15 \mathrm{~min}$ at $4^{\circ} \mathrm{C}$ (SS-34 rotor), the pellet was resuspended in $30 \mathrm{ml}$ denaturating buffer supplemented with $0.5 \%$ Chaps. Undissolved inclusion bodies were removed by ultracentrifugation at $50,000 \mathrm{xg}$ for $30 \mathrm{~min}$ at $4^{\circ} \mathrm{C}$ (SS-34 rotor). Soluble protein fractions (protein purified from inclusion bodies and supernatant) were brought to $10 \mathrm{mM}$ imidazole, combined and loaded on PBS-washed Ni-NTA beads (Sigma-Aldrich). A low concentration of imidazole $(10 \mathrm{mM})$ was included during protein binding and all washing steps to reduce unspecific protein binding to the beads. Beads are always sedimented at $900 \mathrm{xg}$ for $5 \mathrm{~min}$ at room temperature (VWR Micro Star 17R). To allow binding, samples were incubated for 1 hour at room temperature on a rotating wheel, followed by extensive washing ((i) twice with washing buffer $+6 \mathrm{M}$ urea, once with washing buffer $+4 \mathrm{M}$ urea, (ii) twice with TBS +10 $\mathrm{mM}$ EDTA and three times with TBS). All washing steps include 10 min incubation on a rotating wheel (at room temperature for washing steps (i) and at $4{ }^{\circ} \mathrm{C}$ for (ii)), followed by sedimentation of the beads at the respective temperature. Protein concentrations in the supernatant of the pelleted beads were determined after each washing step to control for elution of the protein. To do so, absorption at $280 \mathrm{~nm}$ was measured and protein amount calculated using the protein-specific extinction coefficient (ProtParam at expasy.org). Since the protein was not eluted from the beads, these antigen-coupled beads could be directly used to affinity purify the polyclonal MTM1 antibody (section 2.4.3).

\subsubsection{Affinity purification of polyclonal MTMlantibody from serum}

A polyclonal antibody against human MTM1 was raised by co-injecting two peptides in rabbits at Eurogentec, directed against amino acid 19-33 (1) and amino acid 502-516 (2), two unique stretches within the MTM1 protein coding sequence. Due to the small peptide size, protein-peptide conjugates are used to generate peptide-specific antibodies. In this case KLH (keyhole limpet hemocyanin, a copper-containing non-hem protein from arthropods and mollucs) was used as a carrier protein, coupled to the peptide C-terminally (for 1) and Nterminally (for 2). KLH is especially suited as a carrier as it contains many epitopes to 
stimulate T-helper cells and thereby a B-cell response. To remove reactivity against the carrier protein or linker region, purification of the peptide antibody is necessary.

For preparing the column, the bottom cap was closed, the column filled with $10 \mathrm{ml}$ TBS and the porous disc placed at the bottom. The antigen-coupled beads (see section 2.4.2) were resuspended in TBS from the column, transferred to the column and allowed to settled for $30 \mathrm{~min}$. Immediately after draining the TBS, the column was washed once with $10 \mathrm{ml}$ elution buffer (2 M glycine $\mathrm{pH}$ 2.2) supplemented with fresh $1 \mathrm{ml}$ 1,4-dioxan. The column wash washed with TBS until the $\mathrm{pH}$ was back at 7.4. The antibody serum (first antibody boost from animal 330) was cleared by centrifugation at $4,000 \mathrm{rpm}$ for $30 \mathrm{~min}$ at $4^{\circ} \mathrm{C}$ (Thermo Fisher Scientific, Heraeus Multifuge X1R), separating the cleared serum from sedimented cell debris and a fat film floating on top. After draining the TBS from the column, the bottom cap was carefully closed, the cleared serum added, the top closed with a lid and incubated on a rotating wheel overnight at $4{ }^{\circ} \mathrm{C}$. The next day, the beads were allowed to settle on ice, the serum drained and stored at $-20^{\circ} \mathrm{C}$. Beads were rinsed to the bottom with TBS, a porous disc placed 1-2 mm above the settled beads and PBS was drained, followed by washing once with $0.1 \mathrm{M} \mathrm{Na}$ tetraborate, $500 \mathrm{mM} \mathrm{NaCl}, 0.1 \%$ Tween-20 and three times with TBS. TBS was drained from the column, the bottom cap closed, $750 \mu$ l elution buffer supplemented with 10 $\%$ freshly added 1,4-dioxan added and completely collected in a $1.5 \mathrm{ml}$ tube containing $200 \mu \mathrm{l}$ $1 \mathrm{M}$ Tris $\mathrm{pH} 8.8$ before repeating the elution procedures with $750 \mu \mathrm{PBS}$. After each elution the eluate was properly mixed and if necessary neutralized using $1 \mathrm{M}$ Tris $\mathrm{pH} 8.8$. In total, 13 fractions were collected and protein concentration determined by measuring absorption at 280 nm. All fractions with a significant amount of protein were pooled, concentrated using an Amicon Ultra 15 Falcon (Millipore, pre-washed three times with elution buffer, 30K cut-off, centrifugation at $4,000 \mathrm{xg}, 25 \mathrm{~min}, 4^{\circ} \mathrm{C}$ ) and dialyzed against TBS overnight at $4^{\circ} \mathrm{C}$ (membrane cut-off 6-8 $\mathrm{kDa}$ ). For storage at $-20^{\circ} \mathrm{C}$, the antibody was adjusted to $50 \%$ glycerol and $0.02 \% \mathrm{NaN}_{3}$.

\subsubsection{Preparation of cell extracts from mammalian cell cultures}

For preparation of total cell extracts, cells were grown to $90-100 \%$ confluency, avoiding overgrown cell layers. Cells were placed on ice, washed three times briefly with icecold PBS $+\mathrm{MgCl}_{2}$ and lysis buffer was added, so that the protein concentration of the cell extract lied between $0.3-1 \mathrm{mg} / \mathrm{ml}$. Triton X-100, a non ionic detergent that solubilises integral membrane proteins, peripheral membrane and membrane associated proteins, was present during cell lysis. To complete cell lysis, cell extracts were left on ice for $30 \mathrm{~min}$ and cell 
debris was removed by centrifugation at $13,0000 \mathrm{rpm}$ at $4^{\circ} \mathrm{C}$ for $20 \mathrm{~min}$ (VWR Micro Star $17 \mathrm{R})$.

\subsubsection{Determination of protein concentration using Bradford assay}

Protein concentrations were measured using Bradford assay (Bradford 1976). Bradford is a colorimetric assay, based on a protein-dependent shift in the absorption maximum of Coomassie G250 from $465 \mathrm{~nm}$ to $595 \mathrm{~nm}$. To do so, cell extracts were diluted in PBS to a final volume of $500 \mu \mathrm{l}, 500 \mu \mathrm{l} 2 \mathrm{x}$ Bradford reagent was added, incubated for 5 minutes at room temperature, mixed and absorbance at $595 \mathrm{~nm}$ was measured with a photometer. As blank, 1x Bradford reagent was used. Reliable absorbance values lie between 0.1 and 0.5. Protein concentrations were calculated using a standard curve within the same dynamic range as measured samples.

\subsubsection{Sodiumdodecylsulfate polyacrylamide gel electrophoresis (SDS-PAGE)}

Proteins can be separated based on their molecular weight through Sodiumdodecylsulfate polyacrylamide gel electrophoresis (SDS-PAGE). The negatively charged SDS, together with the reducing agent $\beta$-mercaptoethanol destabilizes the threedimensional protein structure based on non-covalent interactions and disulfide bonds. SDS binds the unfolded polypeptide chain in a ration of $1.4 \mathrm{~g}$ SDS per $1 \mathrm{~g}$ polypeptide. Thus, the netto charge of the unfolded protein is completely masked by the triply charged SDS, creating a constant mass to charge ratio and assuring protein separation according to their molecular weight. The discontinuous SDS-PAGE according to Laemmli (Laemmli 1970) provides a sharp separation of distinct protein bands and consists of a stacking and separating gel with different $\mathrm{pH}$ 's that affects the $\mathrm{pH}$-dependent netto charge of glycine. Thus the lower $\mathrm{pH}$ of the stacking gel assures a slow running of proteins, leading to protein accumulation in sharp bands according the molecular weight. When entering the separating gel distinct protein bands are separated at the higher $\mathrm{pH}$ caused by a fastened running behavior. Composition and percentages of separating and stacking gels are summarized below. Acrylamid can when activated by free radical quickly polymerize to form long chain polymers that are crosslinked by N'-N'-Methylenebisacrylamide forming polyacrylamid gels. Initiation of gel formation by free radicals is provided by ammonium persulfate (APS) and N,N,N',N'tetramethylethylenediamine (TEMED). The percentage of the gel and the pore size are thereby defined by the concentration of Acrylamid/N'-N'-Methylenebisacrylamide (AA/BA) and the amount of APS/TEMED. 
Protein samples were adjusted to a final concentration of 1x SDS sample buffer, boiled for $10 \mathrm{~min}$ at $95^{\circ} \mathrm{C}$, briefly centrifuged at maximal speed in a microcentrifuge and loaded on a SDS-PAGE of appropriate percentage for the separating gel. SDS-PAGEs were run at $15 \mathrm{~mA} /$ SDS-PAGE.

\begin{tabular}{|c|c|c|c|c|}
\hline & \multicolumn{3}{|c|}{ Separating gel } & \multirow{2}{*}{$\begin{array}{l}\text { Stacking gel } \\
3 \%\end{array}$} \\
\hline & $12 \%$ & $10 \%$ & $8 \%$ & \\
\hline $\mathrm{AA} / \mathrm{BA}$ & $2.5 \mathrm{ml}$ & $3 \mathrm{ml}$ & $3.5 \mathrm{ml}$ & $1.25 \mathrm{ml}$ \\
\hline $\mathrm{H}_{2} \mathrm{O}$ & $3 \mathrm{ml}$ & $2.5 \mathrm{ml}$ & $2 \mathrm{ml}$ & $0.625 \mathrm{ml}$ \\
\hline $\begin{array}{l}4 \mathrm{x} \text { stacking or separating } \\
\text { buffer }\end{array}$ & $1.875 \mathrm{ml}$ & $1.875 \mathrm{ml}$ & $1.875 \mathrm{ml}$ & $0.33 \mathrm{ml}$ \\
\hline APS & $75 \mu \mathrm{l}$ & $75 \mu \mathrm{l}$ & $75 \mu \mathrm{l}$ & $37.5 \mu \mathrm{l}$ \\
\hline TEMED & $7.5 \mu \mathrm{l}$ & $7.5 \mu \mathrm{l}$ & $7.5 \mu \mathrm{l}$ & $3.75 \mu \mathrm{l}$ \\
\hline
\end{tabular}

\subsubsection{Immunoblotting}

Immunoblotting or Western blotting (WB) is a highly sensitive method to detect proteins, separated by SDS-PAGE. Therefore proteins, still covered with SDS and thus negatively charged were transferred in an electrical field quantitatively from the SDS-PAGE onto a nitrocellulose membrane (Whatman). Proteins were transferred at $1 \mathrm{~mA} / \mathrm{cm}_{2}$ SDSPAGE for 3 hours onto nitrocellulose membrane using a semi-dry blotting chamber. If high transfer efficiency was required, a tank blotting chamber was used and proteins transferred at $110 \mathrm{~V}$ for $65 \mathrm{~min}$ at $4^{\circ} \mathrm{C}$. Membranes were reversible stained with ponceau stain for 5 minutes at room temperature to check for loaded protein amounts and quantitative transfer. Unspecific ponceau staining was removed by ponceau destain. Membranes were washed briefly with TBS and unspecific antibody binding was blocked with antibody blocking solution for 1 hour at room temperature. Membranes were washed four times for 5 minutes with TBS $+0.05 \%$ Twenn-20 and primary antibodies diluted in antibody dilution solution were incubated overnight at $4^{\circ} \mathrm{C}$. Antibody solutions were recycled and membranes were washed four times for 5 minutes at room temperature with TBS $+0.05 \%$ Tween-20. Secondary antibodies, conjugated to horse radish peroxidase (HRP), were diluted 1:5,000 in blocking solution and incubated for 1 hour at room temperature. Membrane were washed four times for 5 minutes with TBS $+0.05 \%$ Tween-20, once with TBS and developed with enhanced chemiluminescence reagent (ECL, Amersham Bioscience). HRP catalyses the conversion of 
ECL to a chemiluminescent product, that can be detected using light sensitive x-ray films. For quantitative analysis WB development was done using a LI-COR Odyssey Fc imager.

\subsubsection{Membrane fractionation}

siRNA treated HeLa cells were harvested in homogenization buffer and homogenized using a European Molecular Biology Laboratory cell cracker (HGM, Heidelberg, Germany; inner diameter $8.020 \mathrm{~mm}$, ball diameter $8.004 \mathrm{~mm}$, with 12 strokes), followed by three freezethaw cycles in liquid nitrogen. Total cell lysate was collected after centrifugation at $1,000 \mathrm{xg}$ for $5 \mathrm{~min}$ at $4^{\circ} \mathrm{C}$ (VWR Micro Star 17R). To obtain the cytosolic fraction the total cell lysate was centrifuged at 100,000xg for $30 \mathrm{~min}$ at $4^{\circ} \mathrm{C}$ (Beckmann, Optima TXL Ultracentrifuge TLA 100.4), the supernatant was collected and protein concentration and volume determined. The membrane pellet was washed once in homogenization buffer and collected in a volume corresponding to the volume of the cytosol fraction. Equal volumes of total cell lysate, membrane pellet and cytosol fraction were loaded onto a 10\% acrylamide gel for SDS-PAGE followed by immunoblotting. Western blot development was done using a LI-COR Odyssey Fc imager. For Exo70, Sec3, Sec8 and MTM1, protein levels in the total cell lysate were normalized to actin, whereas protein levels in the membrane fraction were normalized to Gadkin. The ratio of membrane to total cell lysate was used to quantify the membrane fraction of Exo70, Sec3, Sec8 and MTM1. For statistical analysis see section 2.3.13.

\subsubsection{Co-Immunoprecipitation}

Expression of HA-tagged PI4K2 $\alpha$ in stably transfected Hek FlpIn cells was induced overnight by addition of doxycylin. As a control untransfected Hek FlpIn cells were used. Cells were harvested in lysis buffer and incubated on ice for $30 \mathrm{~min}$, followed by centrifugation at $43,500 \mathrm{xg}$ for $20 \mathrm{~min}$ at $4{ }^{\circ} \mathrm{C}$ (VWR Micro Star 17R). The supernatant was ultra-centrifuged at $265,000 \mathrm{xg}$ for $15 \mathrm{~min}$ at $4^{\circ} \mathrm{C}$ (TLA 100.4 rotor). HA-matrix beads (Babco, mouse-anti-HA.11) were used to immunoprecipitate HA-tagged PI4K2 $\alpha$. 3-5 $\mathrm{mg} / \mathrm{ml}$ of protein was loaded on $30 \mu 1$ 1:1 washed HA-matrix beads slurry and incubated for 1 hour at $4^{\circ} \mathrm{C}$ on a rotating wheel. Beads were pelleted, washed two times with lysis buffer, followed by two washes with homogenization buffer and bound protein was eluted in $60 \mu 1$ 1x Laemmli sample buffer. Eluates were loaded onto a 10\% acrylamide gel for SDS-PAGE followed by immunoblotting. 


\section{Results}

\subsection{Exocytosis from endosomes is impaired in XLCNM patient cells}

$\mathrm{X}$-linked centronuclear myopathy is a very severe form of congenital myopathy caused by loss of function mutations in MTM1, an endosomal PI 3-phosphatase. MTM1 knockout (KO) mice are born below Mendelian ratio, display impaired growth and progressive myopathy leading to death within 2 to 3 months after birth (Buj-Bello, Laugel et al. 2002). Only XLCNM-patients with a mild form of the disease survive and display muscle as well as nonmuscle phenotypes (Herman, Finegold et al. 1999). This argues for a more general and broader function of MTM1 in muscle and non-muscle tissue. Since developing muscle fibers are characterized by a high degree of membrane turn-over, especially by repeated endo exocytic cycling of surface receptors, muscle cells are very susceptible to membrane-related transport defects. In D. melanogaster MTM1 null animals $\beta 1$-integrin accumulates within the endosomal system. Integrin-based adhesion complexes are not assembled properly and not exposed at the cell surface, thus muscle fibers are not attached to tendons or the extracellular matrix anymore (Ribeiro, Yuan et al. 2011). Furthermore, disorganized membrane compartments and T-tubules were observed in muscle fibers of XLCNM patients (Dowling, Vreede et al. 2009). Thus, we hypothesized that the endosomal accumulation of $\beta 1$-integrin observed upon MTM1 loss-of-function results from defective PI conversion at endosomes and thus defects within the endosomal system.

\subsubsection{B1-integrin accumulates in late and recycling endosomes upon loss of MTM1}

To decipher the molecular mechanisms underlying XLCNM, we made use of the XLCNM patient fibroblast cell line $\mathrm{H} 31$ and the corresponding control cell line (HDFa, human dermal fibroblast from adult healthy individuals). Compared to healthy controls patient-derived H31 cells displayed a strong intracellular accumulation of $\beta 1$-integrin as indicated by immunofluorescence microscopy (Figure 7a). This corresponds nicely to the observed $\beta 1$-integrin accumulations in MTM1 null mutants in D.melanogaster (Ribeiro, Yuan et al. 2011). Further, this phenotype was reproduced by MTM1-depletion in HeLa cells (Figure 7b). In XLCNM-patient cells the intracellular accumulation of $\beta 1$-integrin could be 
rescued to control levels by re-expression of active MTM1 (Figure 7c). These data indicate that intracellular $\beta 1$-integrin accumulation is a direct consequence of MTM1 loss-of-function.

a
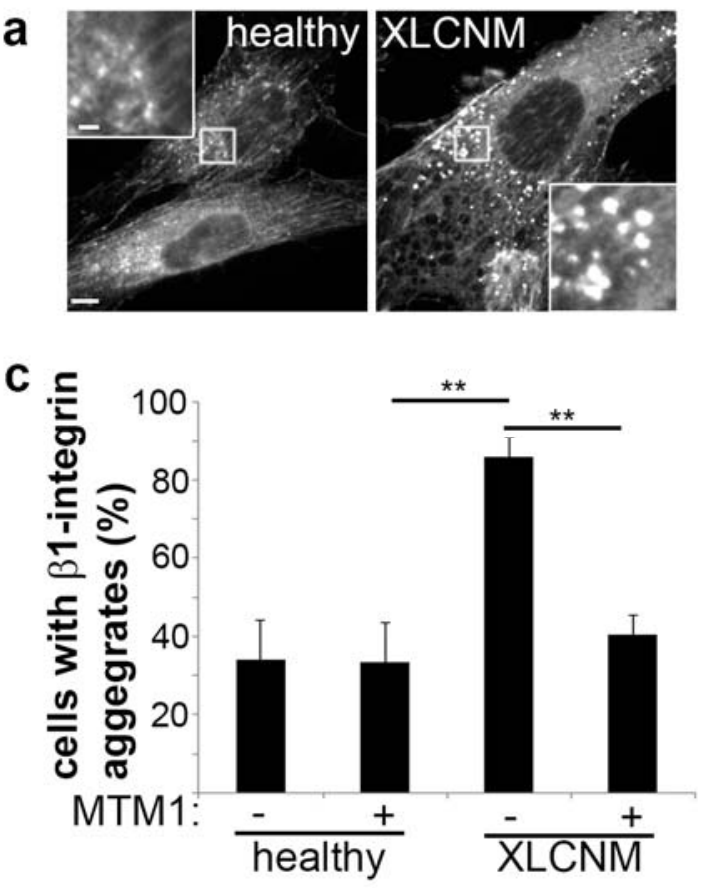

b

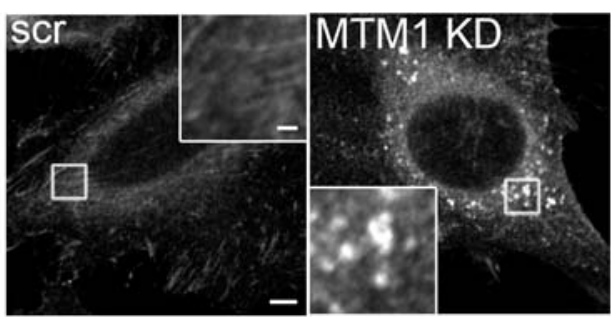

Figure 7: $\beta 1$-integrin accumulation in XLCNM patients can be rescued by re-expression of MTM1

(a, b) Confocal images of $\beta 1$-integrin distribution in XLCNM patient cells (H31), healthy controls (a), or HeLa cells treated with scrambled (scr) siRNA or siRNA against MTM1 (b). Representative images from one of 6 independent experiments are shown. Scale bar, $5 \mu \mathrm{m}$; magnified insets: $1 \mu \mathrm{m}$. (c) Fraction of H31 or HDFa cells displaying $\beta 1$-integrin aggregates. Re-expression of eGFP-MTM1 resolves $\beta 1$-integrin aggregates. Mean $\pm \mathrm{SEM}$, $n=3$ independent experiments with 15-30 images analyzed per condition per experiment, $* * P<0.01$, unpaired, two-tailed $t$-test.

To characterize the endosomal identity of these $\beta 1$-integrin accumulations we tested the co-localization of $\beta 1$-integrin with various endosomal markers in MTM1-depleted and control HeLa cells. Transferrin receptor (TfR) is a nutrient receptor that binds serum transferrin at the cell surface. After clathrin-dependent internalization endocytic vesicle mature to Rab5-positive early/ sorting endosomes and TfR undergoes rapid recycling via exocytosis directly from early endosomes or slow recycling via exocytosis from recycling endosomes (Hsu, Bai et al. 2012). Under normal conditions a very minor, neglectable portion of TfR is sorted to Rab7-positive late endosomes for subsequent degradation. Thus, TfR is an excellent marker for early and recycling endosomes. Several Rabs, including Rab8, are localized to the trans-Golgi-network and regulate biosynthetic, secretory trafficking from the TGN to recycling endosomes and the plasma membrane. Upon depletion of MTM1 $\beta 1$ integrin and TfR showed a strong accumulation within the same recycling endosomal compartment (Figure 8), indicating that progression through the endosomal pathway en route 
to the cell surface is impaired. Hence, defects in cargo exit from recycling endosomes might cause their trapping and accumulation in these endosomes. Furthermore, antibody labeling in eGFP-Rab5, Rab7 and Rab8 expressing MTM1-depleted cells revealed neither accumulation of $\beta 1$-integrin in Rab5-positive early endosomes nor Rab8-labelled TGN. Therefore, we conclude that secretion from the Golgi complex and maturation of early endosomes towards recycling endosomes or late endosomes is not impaired upon loss of MTM1 in XLCNM patients. However, $\beta 1$-integrin strongly accumulated in Rab7-positive late endosomes, indicating that defective exit from recycling endosomes presumably causes mis-sorting of $\beta 1$ integrin into the degradative pathway (Figure 8). In contrast to TfR, $\beta 1$-integrin can be recycled from late endosomes (Rainero and Norman 2013), although the mechanistic details remain poorly characterized. Late endosomal accumulation of $\beta 1$-integrin might therefore be caused by impaired late endosomal recycling or defective lysosomal degradation. In summary, loss of MTM1 in HeLa cells and XLCNM patients causes endosomal accumulation of $\beta 1$-integrin.
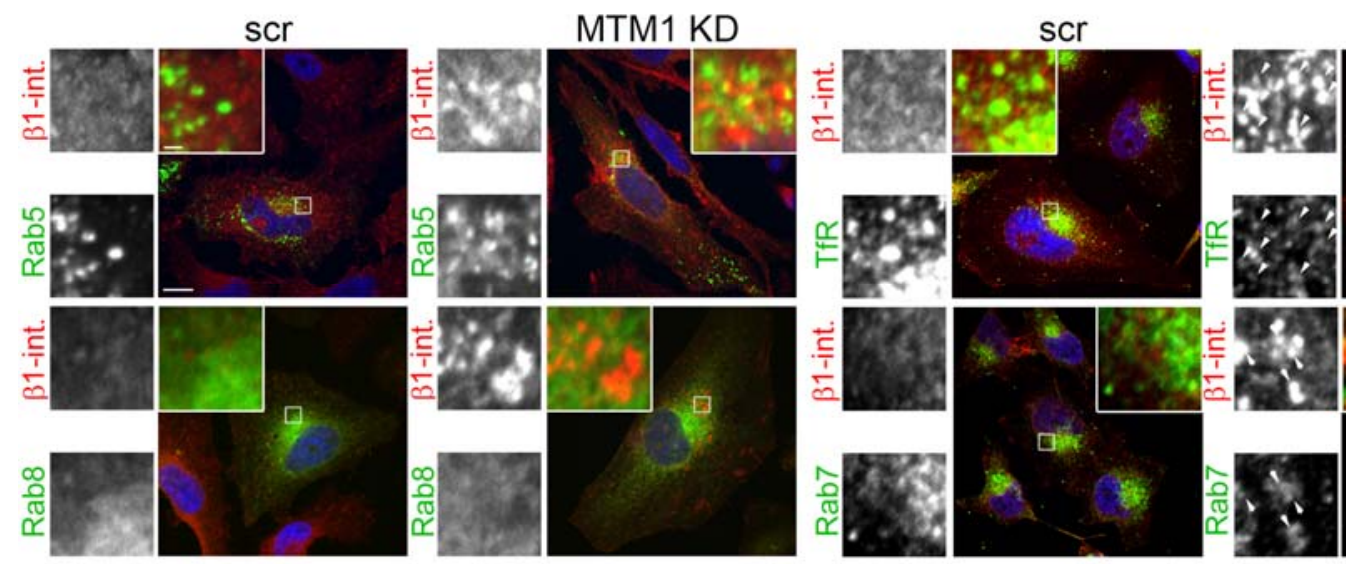

MTM1 KD

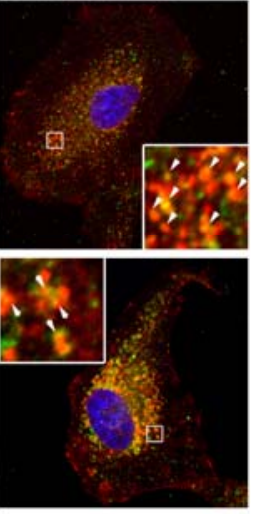

Figure 8: Endosomal accumulation of $\beta 1$-integrin upon loss of MTM1

HeLa cells treated with scrambled (scr) siRNA or depleted of MTM1 (MTM1 KD) expressing eGFP-Rabs (Rab5, Rab7, Rab8) or labeled for TfR were co-labeled using a $\beta 1$-integrin specific antibody. Colocalization of $\beta 1$-integrin with late endosomal Rab7 or recycling endosomal TfR in XLCNM patient cells is indicated by arrowheads. Representative images from one of 2 independent experiments (TfR) or from one experiment with 10-20 images per condition (Rab5, Rab7, Rab8) are shown. Scale bar, $10 \mu \mathrm{m}$; magnified insets, $1 \mu \mathrm{m}$.

\subsubsection{Impaired cargo exit from endosomes due to defective exocytosis}

Based on the accumulation of $\beta 1$-integrin in late and recycling endosomes, compartments where MTM1 was previously found to be localized (Cao, Laporte et al. 2007), we reasoned that cargo exit (i.e. $\beta 1$-integrin or TfR) from endosomes might be defective in XLCNM patients. We further hypothesized that late endosomal accumulation is a secondary effect of 
blocked exit from recycling endosomes. Therefore, we studied the steady-state distribution of TfR in XLCNM patient and healthy control cells in more depth. TfR surface levels were significantly decreased in XLCNM patient cells compared to healthy controls, a phenotype that could be rescued by re-expression of active MTM1. Since total TfR levels were slightly, although not significantly increased in XLCNM patients, the surface receptor levels were always normalized to the total receptor pool for our analyses (Figure 9a, b).

a
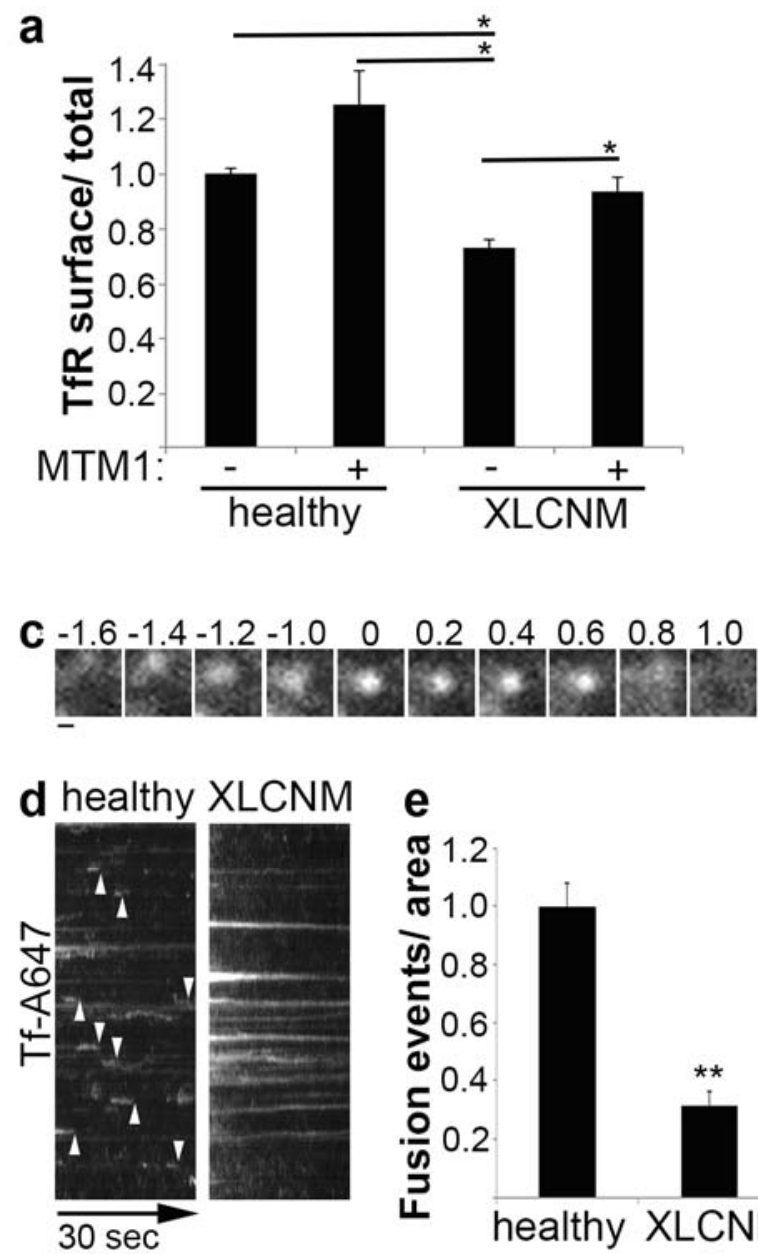

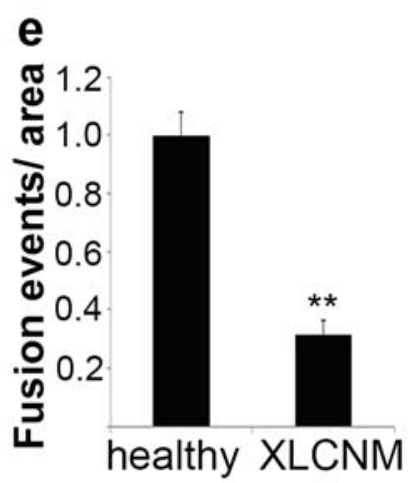

b

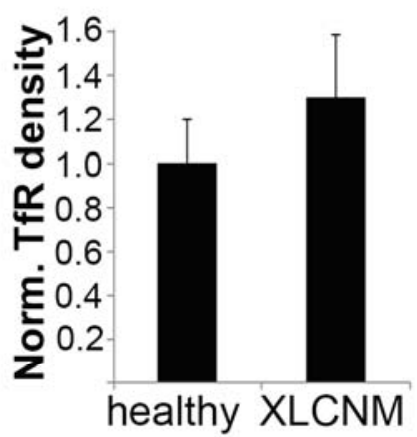

f

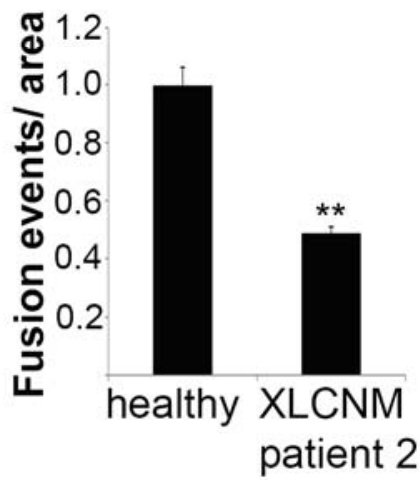

Figure 9: Defective Tf exocytosis in XLCNM patient cells

(a-e) Impaired Tf exocytosis from endosomes in XLCNM H31 patient cells. (a) Ratio of surface/ total TfR in XLCNM patient and healthy control cells expressing eGFP or eGFP-MTM1. Mean \pm SEM, $n=3$ independent experiments with 15-30 images analyzed per condition per experiment, ${ }^{*} P<0.05$, unpaired, two-tailed $t$-test. (b) Quantitative analysis of TfR levels/ cell area of XLCNM patient and healthy control cells (mean \pm SEM, $n=3$ independent experiments with 15-30 images analyzed per condition per experiment). (c-f) TIRF microscopy analysis of Tf-exocytosis in XLCNM patient and healthy control cells: (c) Snapshot of a single exocytic event in healthy controls with characteristic time course of appearance, brightening and spreading of the fluorescent signal. Scale bar: $400 \mathrm{~nm}$. (d) Kymographs of XLCNM patient and healthy control cells over $28 \mu \mathrm{m}$. Arrowheads indicate time of plasma membrane fusion. (e, f) Quantified are Tf exocytic events normalized to cell area in healthy controls and XLCNM-patient cells (e, H31; f, G92-628 patient \#2). Mean \pm SEM, $n=3$ independent experiments with 5 videos analyzed per condition per experiment, $* * P<0.01$, unpaired, two-tailed $t$-test. 
These data indicate that MTM1 is required to maintain the TfR surface pool, presumably by regulating surface delivery of TfR through exocytosis from endosomes.

To address this presumption, we used live-cell TIRF microscopy - ideally suited to monitor processes in close proximity to or at the plasma membrane (Axelrod, Burghardt et al. 1984). After internalization of fluorescently labeled Tf ligand, exocytic events can be observed in the TIRF field and later on identified by their characteristic time course of appearance, brightening and spreading of the fluorescent signal. While in healthy controls Tf containing endosomes were mobile, frequently approached the plasma membrane and released their ligand content upon exocytosis, these events were hardly seen in XLCNM patient cells (Figure 9c, d). Here, Tf-containing endosomes were immobile and accumulated underneath the plasma membrane. This observation was confirmed in a second XLCNM patient cell line, G92-628 (Figure 9e). These data show that endosomal accumulation of recycling cargo, i.e. TfR or $\beta 1$-integrin, results from defective exocytosis from endosomes in XLCNM patients.

\subsection{Tf exocytosis from endosomes requires MTM1-mediated PI(3)P hydrolysis}

To dissect the molecular mechanisms underlying defective endosomal exocytosis in XLCNM patients, we changed to HeLa cells in culture. These cells are easy to manipulate genetically, i.e. by siRNA-mediated knockdown and transient protein expression, and thus represent an ideal model system to study mechanistic details in mammals. In agreement with the data from XLCNM patient cells, MTM1-depletion in HeLa cells caused decreased TfR levels at the cell surface as compared to scrambled siRNA-treated controls (Figure 10a, b). The decreased surface pool of TfR led to a proportional decrease in the capacity of MTM1depleted HeLa cells to take up Tf-ligand (Tf uptake and Tf uptake/ surface, Figure 10b). These results could be confirmed by using radioactively labeled Tf ligand to quantify $\mathrm{Tf}$ internalization and surface accessibility (Figure 10e-g). The kinetics of $\mathrm{Tf}$ internalization, calculated as the slope of the linear regression line $\left(1 / K_{m}\right)$, were unaltered upon MTM1 depletion with a slight tendency to $\mathrm{Tf}$ accumulation at later time points (8min). This accumulation is presumably caused by a combination of continuous, unaltered uptake and inhibited Tf recycling due to the loss of MTM1. Matching this assumption, it was shown that recycling starts within 5 min after internalization from perinuclear recycling endosomes 
(Ciechanover, Schwartz et al. 1983). Total TfR levels, as measured by immunocytochemistry and flow cytometry, were unaltered in MTM1-depleted cells when compared to controls (Figure 10c, d). These data indicate that MTM1 is required for surface delivery of endosomal cargo, i.e. TfR, while ligand internalization remains unperturbed.
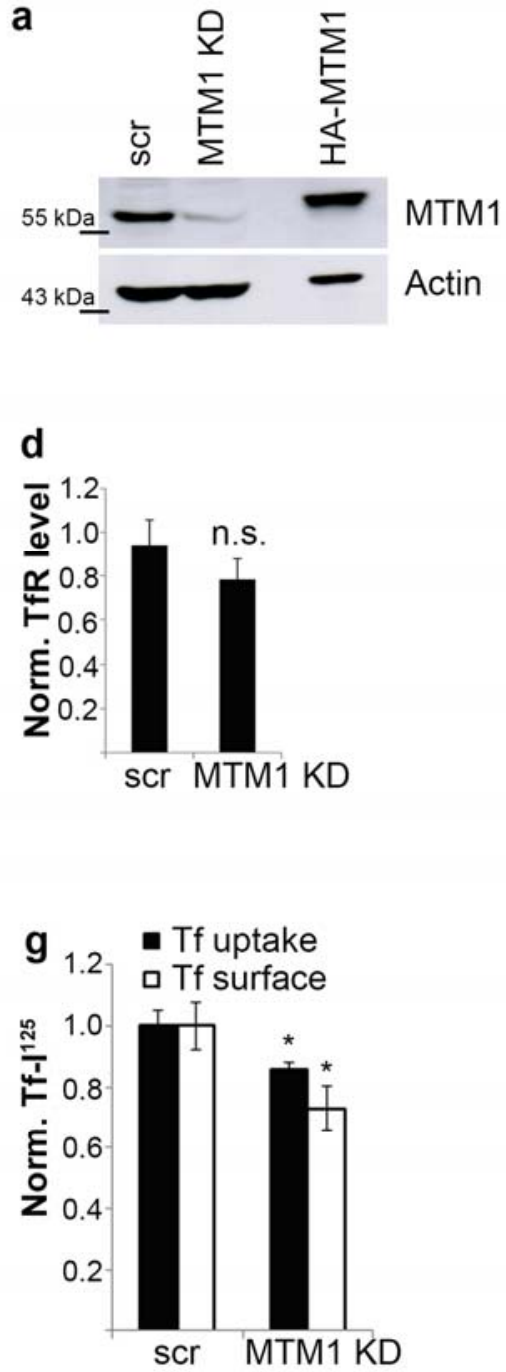
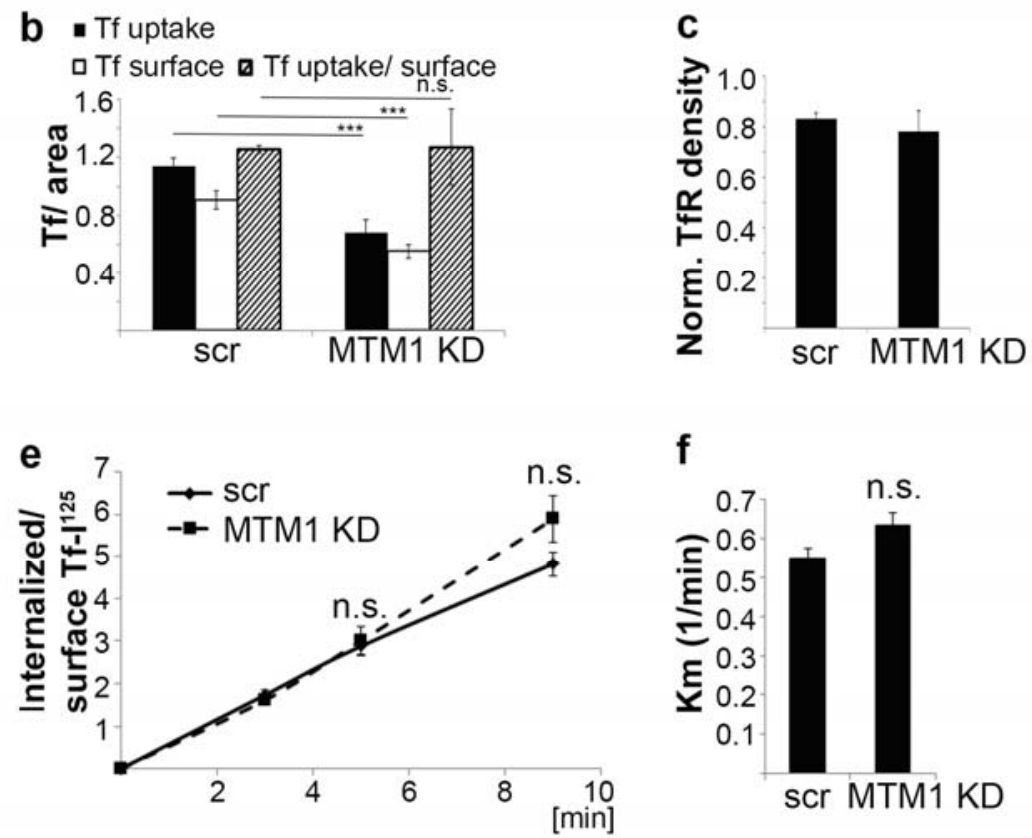

Figure 10: Decreased TfR surface level as a readout for defective TfR recycling

(a) Efficient siRNA-mediated depletion of MTM1 from HeLa cells as demonstrated by immunoblot analysis. Lysates of HeLa cells expressing HA-MTM1 were taken as a control to assure MTM1 antibody specificity. (b) Tf uptake, surface Tf levels and ratio of uptake/ surface-bound Tf in HeLa cells depleted of MTM1 (MTM1 KD) compared to scrambled siRNA-treated controls (scr). Means \pm SEM, $n=3$ independent experiments with 15-30 images analyzed per condition per experiment, $* * * P<0.001$, n.s.: non-significant, unpaired, two-tailed $t$-test. (c,d) Quantitative analysis of TfR levels by confocal imaging (TfR levels/ area; e) or flow cytometry (TfR levels/ cell; f) of HeLa cells treated with scrambled (scr) control siRNA or depleted of MTM1 (MTM1 KD) (mean \pm SEM, $n=3$ independent experiments, n.s.: non-significant, unpaired, two-tailed $t$-test). (e-g) Time course of $\mathrm{Tf}$ internalization was measured radioactively and the ratio between $\mathrm{Tf}$ uptake and surface $\mathrm{Tf}$ levels over time was quantified in HeLa cells depleted of MTM1 compared to scrambled siRNA-treated controls. While Tf endocytic rates remained unchanged in MTM1 KD cells (e,f), Tf uptake and surface level were significantly decreased in MTM1-depleted cells (g), indicative of defective Tf recycling upon loss of MTM1. Means \pm SEM, $n=4$ independent experiments, each experiment done in technical triplicates, ${ }^{*} P<0.05$, n.s.: non-significant, unpaired, two-tailed $t$-test. 
Consistent with reduced Tf exocytosis in XLCNM patient cells, exocytic events were also observed less frequently in MTM1-depleted HeLa cells. This phenotype depends on the 3-phosphatase activity of MTM1 as expression of siRNA-resistant, wild-type (wt) MTM1 but not of a phosphatase-inactive mutant (C375S) MTM1 rescued the exocytic defect upon loss of MTM1 (Figure 11). In the C375S mutant, the core cystein of the $\mathrm{CX}_{5} \mathrm{R}$-phosphatase motive is mutated and renders the enzyme catalytically inactive, as previously shown (Taylor, Maehama et al. 2000).

To confirm MTM1-phosphatase activity towards PI(3)P, cellular PI(3)P level were monitored by immunocytochemical stainings in cells expressing wild-type or mutant (C375S) MTM1. While PI(3)P levels were drastically reduced upon expression of the wild-type enzyme, PI(3)P levels were unaltered in cells expressing C375S mutant MTM1 (see Figure $20 \mathrm{~d}$ discussed on page $80-81$ ). Together, these data indicate that the exocytic defect observed upon MTM1 depletion is most likely caused by an elevation of cellular PI(3)P levels.
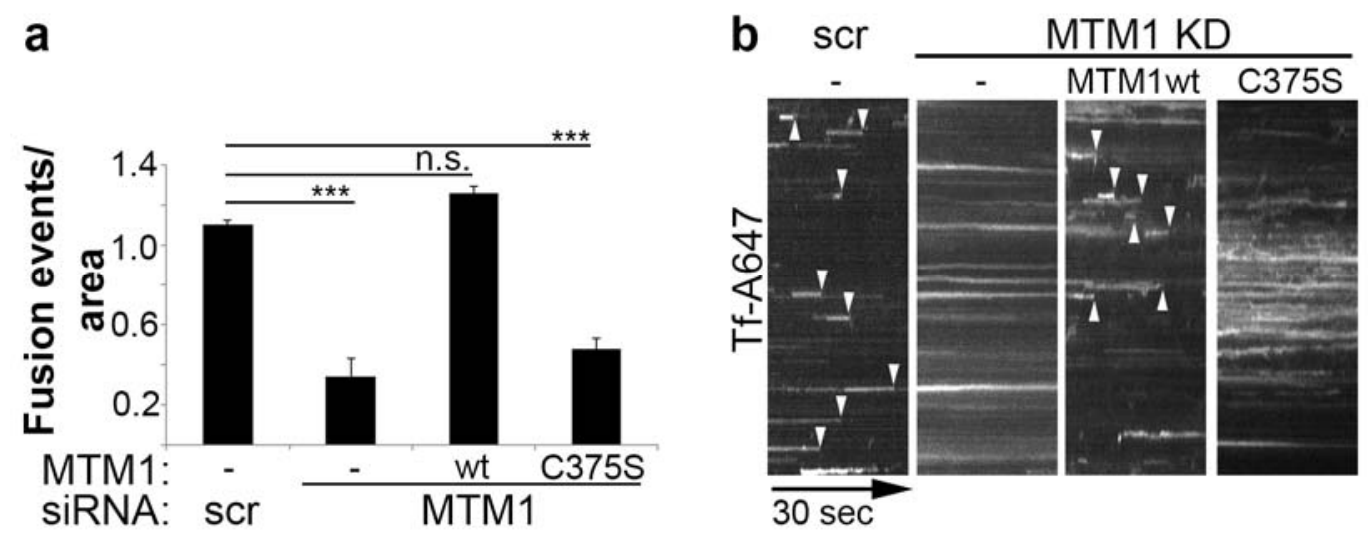

Figure 11: Tf exocytic defect upon loss of MTM1

(a) Impaired Tf exocytosis monitored by TIRF microscopy in HeLa cells depleted of MTM1 (MTM1 KD) compared to scrambled siRNA-treated controls (scr). Re-expression of wild-type (wt) eGFP-MTM1, but not catalytically inactive MTM1 C375S or eGFP rescues defective Tf exocytosis. Means \pm SEM, $n=3$ independent experiments with 5 videos analyzed per condition per experiment, ${ }^{* * *} P<0.001$, unpaired, two-tailed $t$-test. (b) Kymographs of $\mathrm{Tf}$ fluorescence intensity over $28 \mu \mathrm{m}$. Arrowheads indicate time of plasma membrane fusion.

\subsubsection{The subcellular distribution of TfR is altered upon loss of MTM1}

To understand the underlying mechanism of how surface-delivery of endosomal cargo is regulated by MTM1 we analyzed the subcellular distribution of TfR. In control cells treated with scrambled-siRNA TfR was predominantly found in the perinuclear region together with its ligand, consistent with its localization of perinuclear early and recycling endosomes. This perinuclear TfR pool was lost upon MTM1 depletion, while stalled TfR-/ Tf-containing 
endosomes accumulated underneath the plasma membrane (Figure 12a-c).
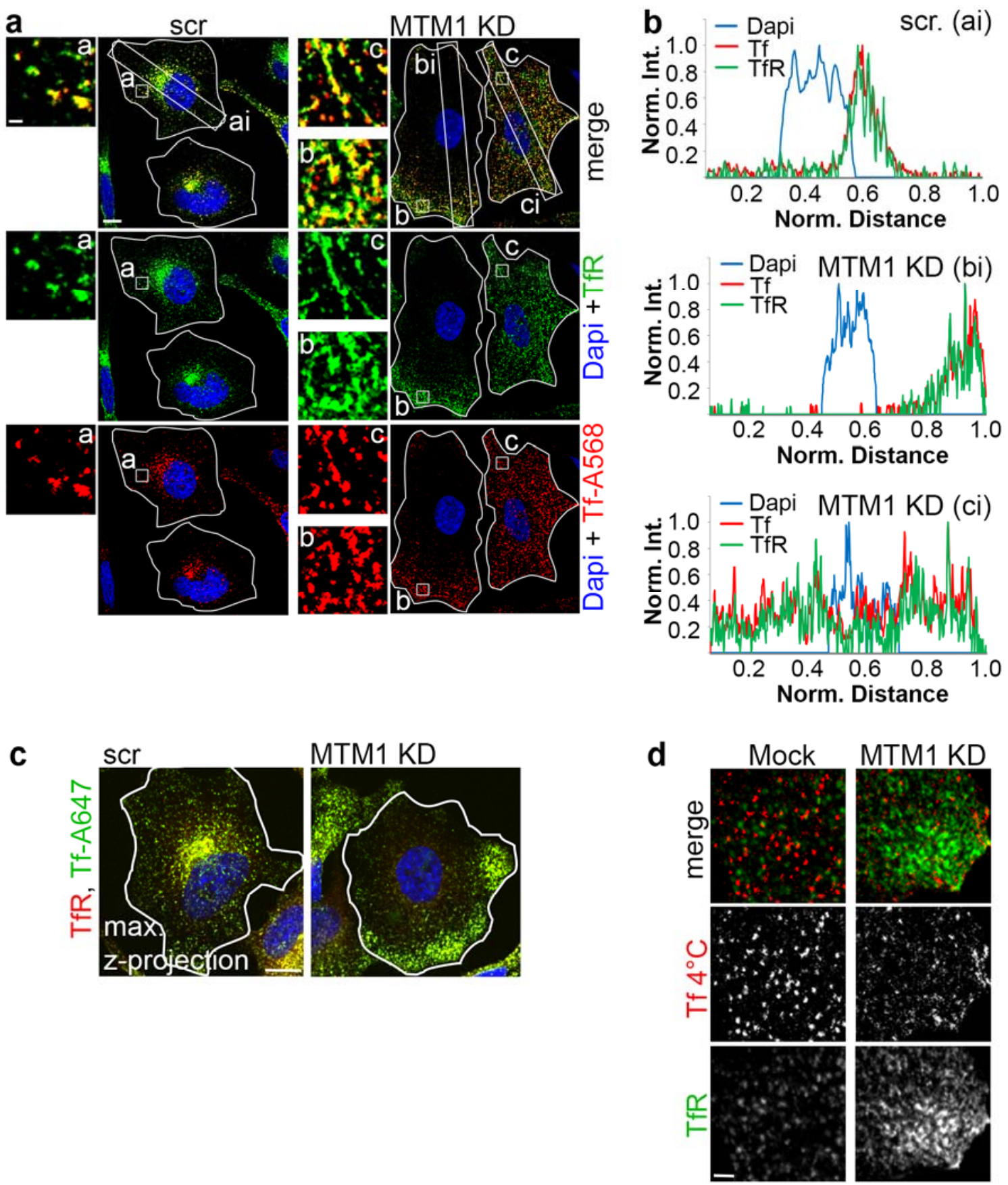

Figure 12: Accumulation of subplasmalemmal TfR-/ Tf-ligand containing endosomes in MTM1-depleted cells

(a) Colocalization between internalized Tf and TfR in MTM1-depleted cells revealed by confocal imaging. Representative images from one of 3 independent experiments are shown. Scale bar, $10 \mu \mathrm{m}$; magnified insets: 1 $\mu \mathrm{m}$. (a,b) Averaged line scans over the marked area in the confocal images illustrate perinuclear accumulation of $\mathrm{TfR}$ in controls (a, b-ai) and accumulation of TfR at the cell periphery (a, b-bi) or cytosolic dispersion (a, b-ci) in MTM1-depleted cells. (c) Maximum intensity projections of z-stacks acquired by confocal imaging confirm the perinuclear accumulation of Tf-ligand and TfR in controls and accumulation at the cell periphery in MTM1depleted cells. Representative images from one experiment with 10 images per condition are shown. Scale bar, 5 $\mu \mathrm{m}$. (d) Surface TfR labeled with Tf-Alexa647 (at $4^{\circ} \mathrm{C}$ ) does not colocalize with intracellular TfR in control or MTM1-depleted HeLa cells. Representative images from one of 2 independent experiments are shown. Scale bar, $2 \mu \mathrm{m}$. 
Endosomal identity of the TfR accumulations was confirmed, as these structures were clearly distinct from the surface TfR-pool (Figure 12d). Furthermore, the nearly perfect colocalization between TfR and internalized Tf ligand in MTM1-depleted cells indicates that although TfR-positive endosomes are unable to undergo exocytosis, these endosomes remain accessible to internalized Tf-ligand (Figure 12a-c). A subpopulation of MTM1-depleted cells displayed a complete dispersion of TfR, mostly in combination with a prominent tubulation of the TfR-compartment (Figure 12b, ci). The recycling compartment is well known to form endosomal tubules for cargo- and adaptor-specific sorting. The accumulation of such tubules might be caused by the accumulation of tubule-stabilizing adaptor proteins as discussed later (section 3.3.1). However, since this strong tubulation was observed only under very fast and stringent fixation conditions (PFA at $37^{\circ} \mathrm{C}, 10 \mathrm{~min}$ ) and turned out to be less reproducible, we focused on the subplasmalemmal accumulations of TfR in our subsequent experiments. These TfR clusters were found in the majority of cells under various experimental conditions.

Compared to the short-term effects observed upon depletion of MTM1 in cell culture, long-term loss-of-function in XLCNM-patients and patient-derived cell lines might have additional defects in order to cope with or compensate for defective exocytic trafficking. XLCNM is mostly caused by complete loss of MTM1 or mutations that render MTM1 phosphatase inactive. However, not all known mutations lie within the PTP-domain and likely effect protein-protein or protein-lipid interactions as well. To delineate how the observed misslocalization of TfR in MTM1-depleted HeLa cells correlates with MTM1 phosphatase activity and phenotypic changes in XLCNM, we re-expressed active, wild-type MTM1, phosphatase-inactive mutant (C375S) and XLCNM-associated MTM1 mutants (P205L, R241L and Y397C, previously shown to render the phosphatase inactive (Taylor, Maehama et al. 2000)) in HeLa cells depleted of the endogenous enzyme. Although all of these mutations impair MTM1 phosphatase activity, only Tyr-397 lies within the PTP-domain, while Pro-205 and Arg-241 are part of the RID-domain, involved in membrane recruitment and proteinprotein interactions. Only active MTM1 was able to restore normal TfR localization. This clearly indicates that MTM1 3-phosphatase activity is needed for normal TfR-trafficking and exocytosis while defective exocytic trafficking in XLCNM patient cells results from MTM1 loss-of-function (Figure 13). Nevertheless, we could not exclude additional long-term defects in XLCNM patients and have only tested a small subset of XLCNM-patient mutations. The inability of MTM1 comprising these XLCNM-associated mutations in rescuing implicates that defective exocytic trafficking is the primary cause of XLCNM in humans. 


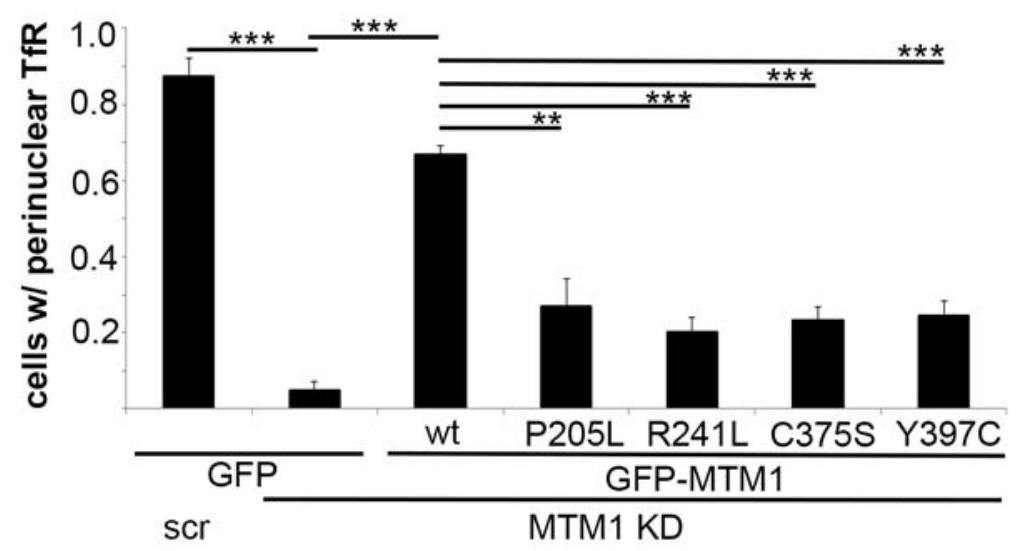

Figure 13: XLCNM-mutants of MTM1 are unable to rescue perinuclear loss of TfR in MTM1-depleted cells

Re-expression of eGFP-MTM1 wt but not C375S or XLCNM patient mutants (P205L, R241L, Y397C) restores perinuclear TfR localization in MTM1-depleted cells. Normalized fraction of cells with perinuclear TfR was quantified (mean $\pm \mathrm{SEM}, n=3$ independent experiments with 15-30 images analyzed per condition per experiment, ${ }^{*} P<0.01,{ }^{* * *} P<0.001$, unpaired, two-tailed $t$-test).

\subsubsection{Endosomal trafficking and cargo sorting in MTM1-depleted cells}

Endosomal membranes form a highly dynamic and interconnected system in which individual components are constantly exchanged between sub-cellular compartments by vesicular transport. Upon internalization from the plasma membrane endosomal cargo converges at the early endosome and is subsequently sorted for recycling or degradation to recycling and late endosomes respectively. Further, at the level of recycling endosomes cargo for retrograde transport to the TGN has to be distinguished from cargo destined for exocytic delivery to the cell surface. This distinction is aided by cargo-specific adaptors (Hsu and Prekeris 2010). To analyze whether MTM1-depleted cells suffer from broader and more complex trafficking problems we studied various intracellular trafficking routes involving endosomes other than endosomal exocytosis. Degradative sorting of EGF as well as total EGFR levels were found to be unaffected by loss of MTM1 (Figure $14 \mathrm{a}, \mathrm{b}$ ), thus excluding a defect in progression from early to late endosomes and subsequent lysosomal degradation. Further, the clathrin-independent internalization of Cholera toxin $(\mathrm{Ctx})$, retrograde transport of Ctx from endosomes to the TGN and consecutive secretion from the Golgi complex were unaffected in MTM1-depleted cells (Figure $14 \mathrm{c}, \mathrm{f}-\mathrm{h}$ ). 

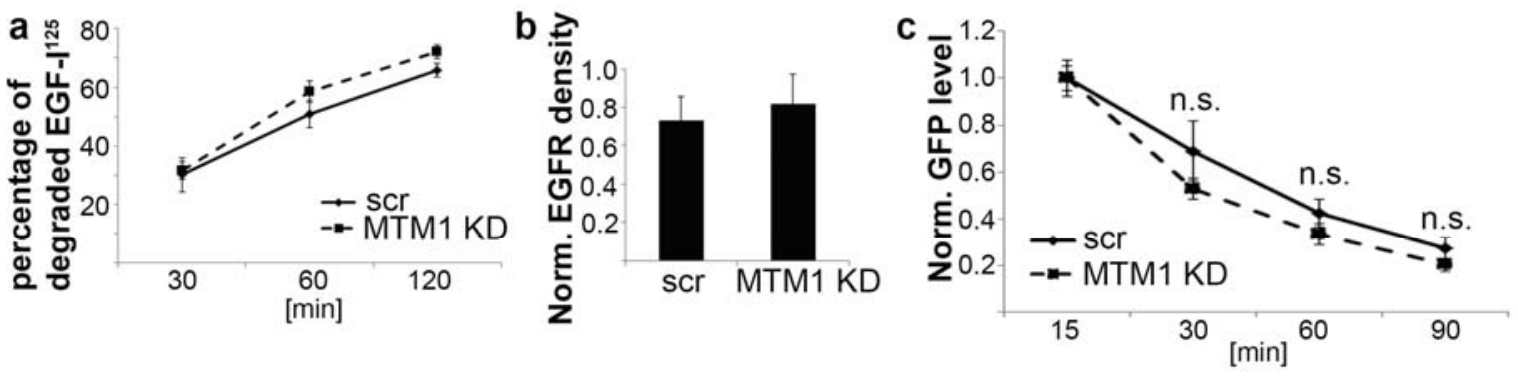

d

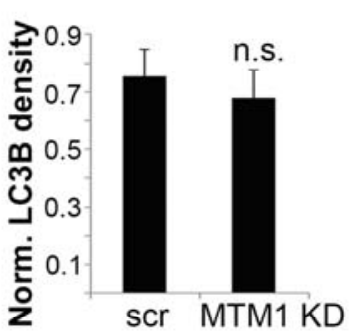

f
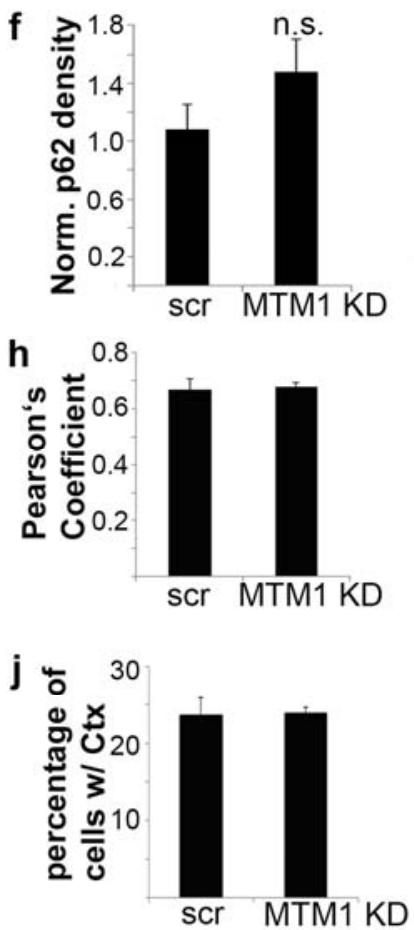

e
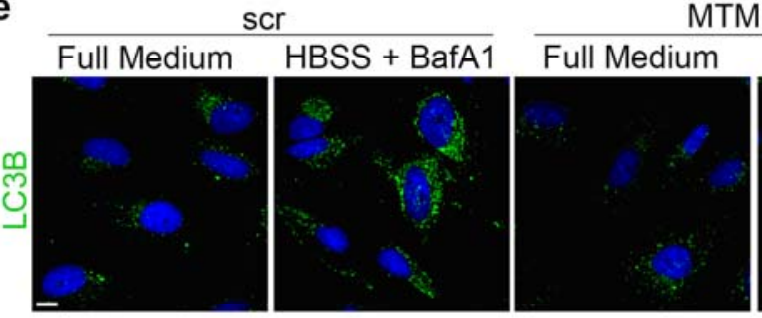

g

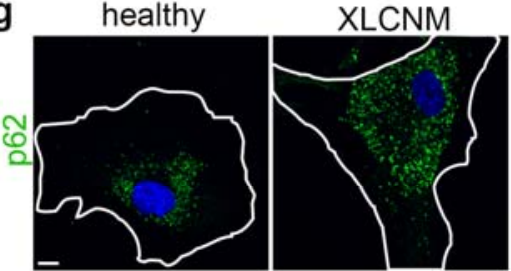

i

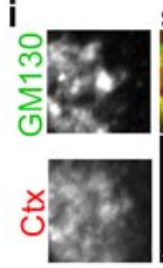

MTM1KD

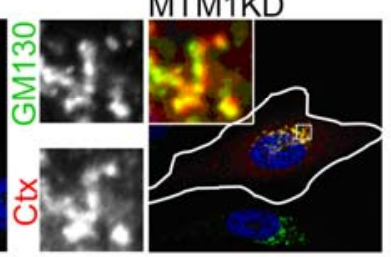

Figure 14: Endosomal trafficking in MTM1-depleted cells

(a) EGF degradation monitored over $120 \mathrm{~min}$ in HeLa cells using radioactive $\mathrm{I}^{125}$-EGF was unaltered upon loss of MTM1 compared to scrambled treated controls (mean \pm SEM, $n=3$ independent experiments, each experiment done in technical triplicates). (b) Quantitative analysis of EGFR levels normalized to cell area in HeLa cells treated with scrambled (scr) control siRNA or depleted of MTM1 (MTM1 KD) (EGFR/ area; mean \pm SEM, $n=3$ independent experiments with 15-30 images analyzed per condition per experiment). (c) Secretion of GFP-reporter construct from the Golgi complex is not affected in MTM1-depleted C1 cells. 15 min after initiating secretion GFP is enriched in the Golgi complex. GFP levels were normalized to cell number and Golgi content (mean \pm SEM, $n=3$ independent experiments with 15-30 images analyzed per condition per experiment, n.s.: non-significant, unpaired, two tailed $t$-test). (d) LC3B level remain unchanged in HeLa cells depleted of MTM1 compared to scrambled control siRNA treated HeLa cells (LC3B/ area; mean \pm SEM, $n=3$ independent experiments with 15-30 images analyzed per condition per experiment, n.s.: non-significant, unpaired, two tailed $t$-test). (e) Autophagy can be induced in HeLa cells depleted of MTM1 by starvation and BafA1 treatment, monitored by LC3B-specific antibody labeling. Representative images from one of 2 independent experiments are shown. Scale bar, $10 \mu \mathrm{m}$. (f) p62 level remain unchanged in HeLa cells depleted of MTM1 compared to scrambled control siRNA treated HeLa cells (p62/ area; mean \pm SEM, $n=4$ independent experiments with 15-30 images analyzed per condition per experiment, n.s.: non-significant, unpaired, two tailed $t$-test). (g) Basal 
autophagy is upregulated in fibroblasts from XLCNM-patients (H31) compared to healthy controls, monitored by p62-specific antibody labeling. Representative images from one experiments with 10-20 images per conditon are shown. Scale bar, $10 \mu \mathrm{m}$. (h,i) Internalized Cholera toxin (Ctx) is retrogradely transported to the Golgi complex in HeLa cells treated with scrambled (scr) siRNA or depleted of MTM1 (MTM1 KD). (h) Quantified are Pearson's Coefficients between internalized Ctx-CF568 and the Golgi complex, assessed by GM130-specific antibody labeling (mean \pm SEM, $n=3$ independent experiments with 15-30 images analyzed per condition per experiment). (i) Representative images from one of 3 independent experiments are shown. Scale bar, $10 \mu \mathrm{m}$; magnified insets, $1 \mu \mathrm{m}$. (j) Cholera toxin uptake quantified as the percentage of cells with internalized Ctx is unchanged upon loss of MTM1 (mean $\pm \mathrm{SEM}, n=3$ independent experiments with 15-30 images analyzed per condition per experiment).

PI(3)P generation and removal play a key role in autophagy initiation and completion, respectively (Cebollero, van der Vaart et al. 2012; Dall'Armi, Devereaux et al. 2013). Commonly used autophagy markers are among others: (a) p62, an autophagy adaptor linking polyubiquitylated, misfolded and aggregated proteins or dysfunctional organelles to LC3 for their removal through autophagy and (b) mammalian LC3 (microtubule-associated protein 1 light chain 3), the homologue to Atg8 in yeast. Upon autophagy induction, cytoplasmic LC3-I is conjugated to phosphatidylethanolamine (PE) and integrated into autophagosomal membranes with roles in cargo selection as well as membrane tethering and fusion (Mizushima, Levine et al. 2008). Because elevated levels of the autophagic markers LC3 and p62 were observed in MTM1 KO mice (Al-Qusairi, Prokic et al. 2013; Fetalvero, Yu et al. 2013), we looked for changes in autophagy markers and autophagy initiation upon loss of MTM1 in HeLa cells. Despite a minor, non significant elevation in total p62 level (Figure 14 f), total LC3B level and autophagy induction were unperturbed in MTM1-depleted HeLa cells as compared to controls (Figure $14 \mathrm{~d}$, e). Such differences between the in vivo mouse model (long-term loss of MTM1) and cell culture (short-term loss of MTM1) could be due to accumulating effects over time. Further, compensatory up-regulation of alternative transport routes, i.e. late endosomal/ lysosomal sorting and autophagic flux, might occur when exocytic exit from endosomes is blocked in MTM1-null animals. In agreement with this hypothesis, p62 levels were found upregulated in XLCNM patient-derived cells (Figure $14 \mathrm{~g}$ ).

Taken together these data show the predominant involvement of MTM1 in exocytosis of recycling endosomal cargo and exclude a more complex trafficking problem upon loss of MTM1 in cell culture.

\subsubsection{Other Myotubularin family members in TfR exocytosis}

MTM1 belongs to a large family of conserved proteins (MTM1, Myotubularin related (MTMR) 1-14), comprising active and inactive PI 3-phosphatases (Amoasii, Hnia et al. 2012). To address potential redundancy within the myotubularin family, TfR localization and 
exocytosis were monitored upon depletion of other, catalytically active myotubularins (MTMR1, MTMR2, MTMR4, MTMR7).

a
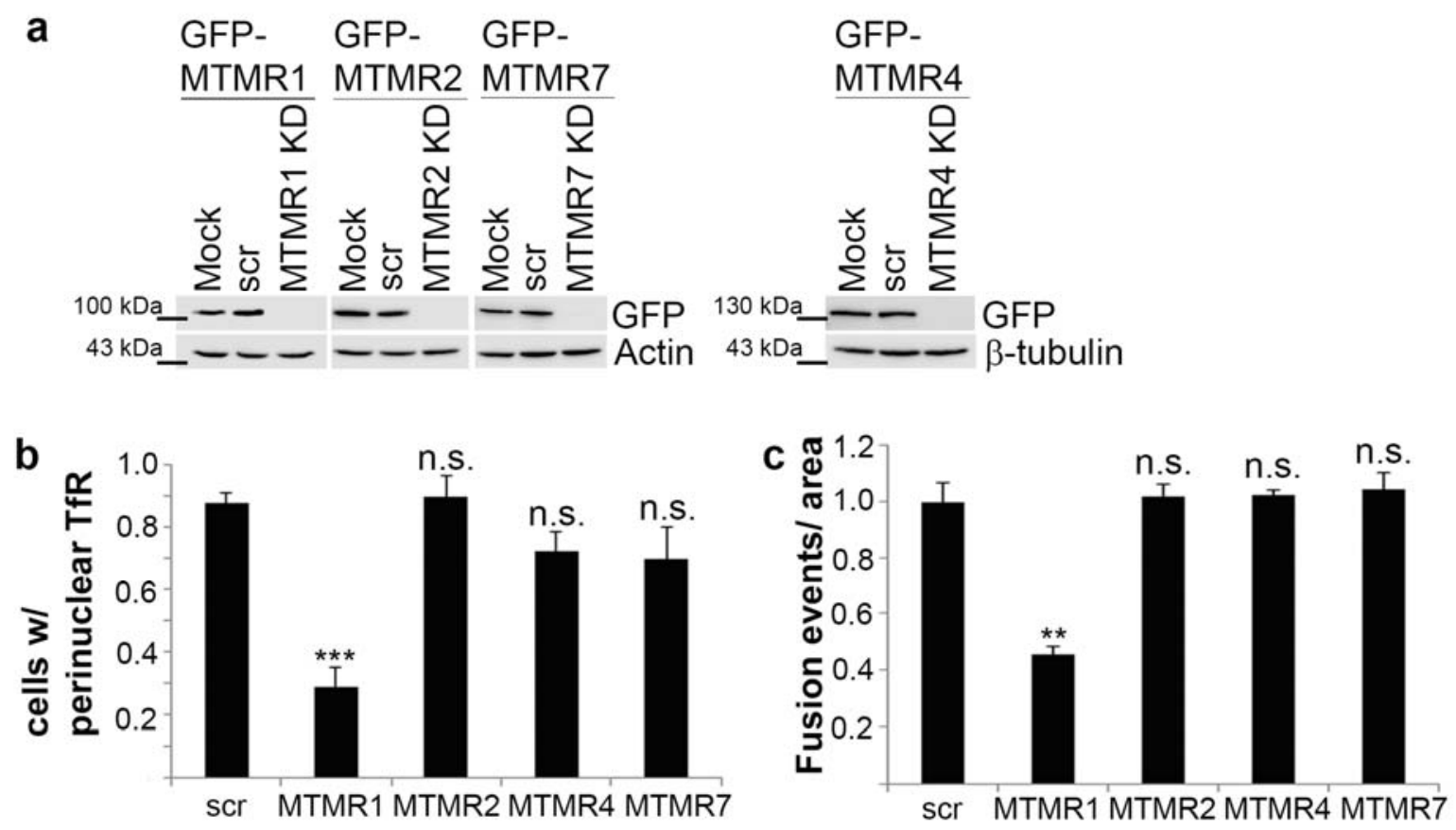

Figure 15: Effects of depletion of other MTM family members

(a) Efficient siRNA-mediated depletion of eGFP-tagged catalytically active Myotubularin related (MTMR) proteins (MTMR1, MTMR2, MTMR4, MTMR7) from HeLa cells as demonstrated by immunoblot analysis. (b) Depletion of MTMR1 but not MTMR2, MTMR4 or MTMR7 phenocopied TfR misslocalization observed in MTM1-depleted HeLa cells (mean \pm SEM, $n=3$ independent experiments with 15-30 images analyzed per condition per experiment, $* * * P<0.001$, unpaired, two-tailed $t$-test). (c) Inhibition of Tf exocytosis in cells depleted of MTMR1 but not MTMR2, MTMR4 or MTMR7 compared to scrambled (scr) siRNA-treated controls (Tf exocytic events/ area; mean \pm SEM, $n=3$ independent experiments with 5 videos analyzed per condition per experiment, $* * P<0.01$, unpaired, two-tailed $t$-test).

A similar, although less severe phenotype was seen upon depletion of the closely related PI 3-phosphatase MTMR1, an enzyme of so far unknown function, while loss of MTMR2 (a PI(3)P and PI(3,5) $\mathrm{P}_{2}$ 3-phosphatase, localized to early and late endosomes and implicated in degradative sorting (Cao, Backer et al. 2008)), MTMR4 (a PI(3)P 3phosphatase, localized to early and recycling endosomes and implicated in endosomal sorting (Naughtin, Sheffield et al. 2010)) or MTMR7 (a PI(3)P and I(1,3) $\mathrm{P}_{2}$ 3-phospahatse, associated with Golgi-like structures (Mochizuki and Majerus 2003)) had no effect on TfR distribution or exocytosis (Figure 15). One might speculate that differences in tissue-specific and developmentally regulated expression patterns account for the need of MTM1 and MTMR1 in vivo. Noteworthy, RNA-levels of MTMR1 were unaltered in muscle tissue from MTM1-null mice, indicating that MTMR1 does not compensate for MTM1 loss-of-function in muscle fibers (Al-Qusairi, Prokic et al. 2013). Due to its localization to Rab5-positive early and 
Rab11-positive recycling endosomes via its PI(3)P-binding FYVE-domain, MTMR4 might be a candidate for functional substitution of MTM1. In accordance with our data, TfR recycling remains unperturbed upon loss of MTMR4. However, sorting of TfR from early to recycling endosomes is impaired while over-expression of active MTMR4 has dominant negative effects on TfR recycling (Naughtin, Sheffield et al. 2010).

Thus, most myotubularin family members are dispensable for endosomal exocytosis and most likely function elsewhere in the endolysosomal transport system. However, MTMR1 functionally overlaps with MTM1.

\subsection{Endosomal accumulation of PI(3)P and PI(3)P effector proteins inhibits exocytosis from endosomes}

The accumulation of post-endocytic Tf-positive endosomes underneath the plasma membrane, together with the depletion of the perinuclear TfR pool upon loss of MTM1 clearly indicates defective endosomal recycling. Nevertheless, up to this point it remained unclear whether loss of MTM1 affects recycling from peripheral early endosomes, progression from early to recycling endosomes, or exocytosis from - under control conditions - perinuclear recycling endosomes. To tackle this fundamental question we characterized the nature of the accumulated sub-plasma membrane endosomes in more detail.

\subsubsection{Sub-plasma membrane early and recycling endosomes accumulate upon loss of MTM1}

Small GTPases, including members of the Rab family are ideally suited to discriminate between endosomal subpopulations as - together with different PI species - they serve as spatial landmarks conferring membrane identity (Behnia and Munro 2005). Peripheral TfR-cluster in MTM1-depleted HeLa cells were highly enriched in early endosomal markers, i.e. early endosomal antigen 1 (EEA1), Rab4 and Rab5, and recycling endosomal marker, i.e. Rab11 and Rab14 (Figure 16a, c). However, the degree of colocalization was unchanged when compared to scrambled siRNA-treated controls (Figure $16 \mathrm{~b}, \mathrm{~d})$. Furthermore, early and recycling endosomes remained clearly separated in MTM1depleted and control cells, indicating that progression from early to recycling endosomes together with compartmental identity and segregation remained unaffected (Figure 17). 

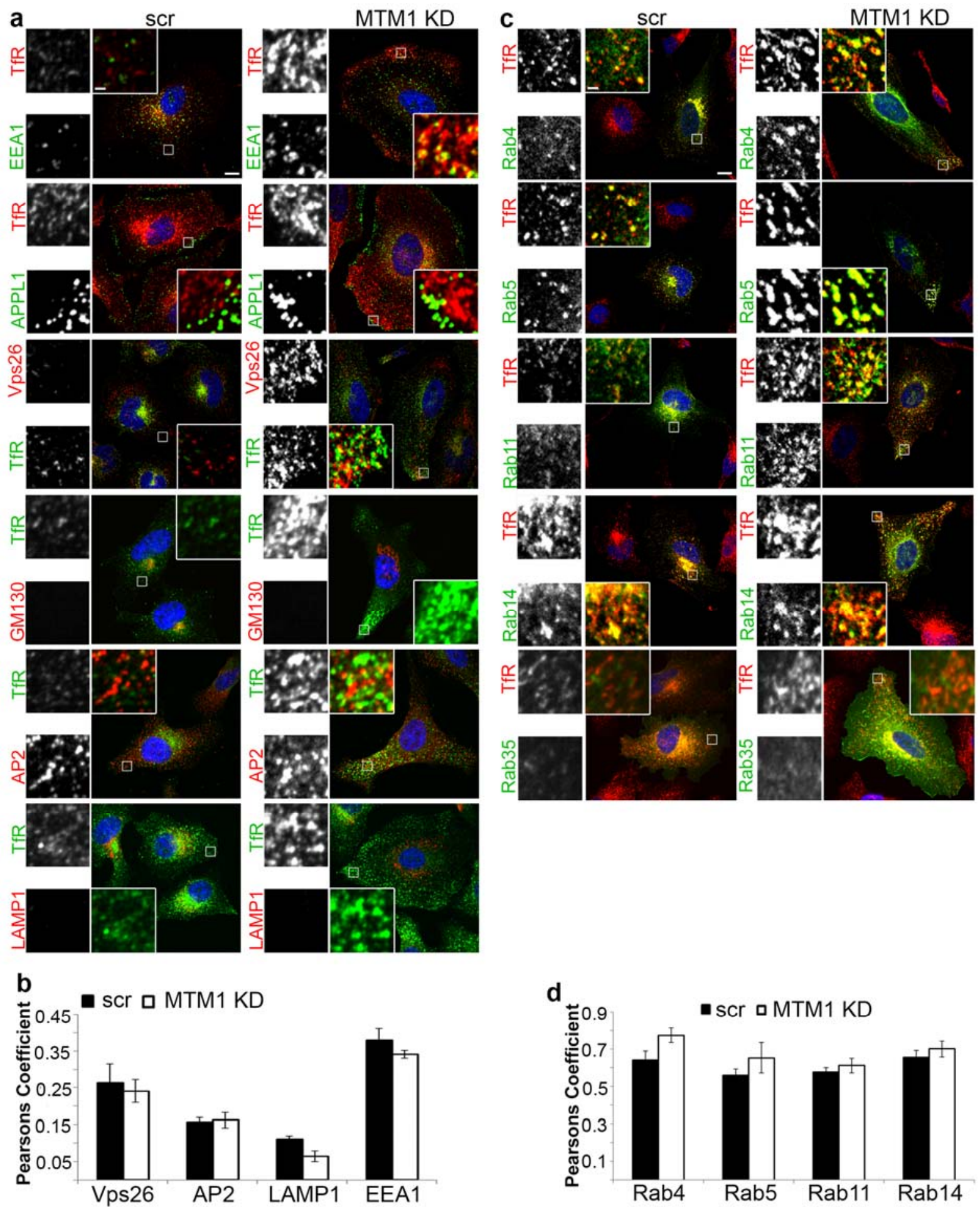

Figure 16: Endosomal compartments in MTM1-depleted cells

(a,b) HeLa cells depleted of MTM1 were labeled for the indicated protein by specific antibodies. EEA1 localizes to sub-plasma membrane TfR accumulations, distinct from sites containing the endocytic adaptor AP-2 or the retromer component Vps26. APPL1 and LAMP1 did not localize to TfR accumulations in MTM1-depleted cells. No changes in the GM130-containing cis-Golgi compartment were observed. Representative images from one of 3 independent experiments are shown. Scale bar, $10 \mu \mathrm{m}$; magnified insets, $1 \mu \mathrm{m}$. (b) Quantification of colocalization between the indicated protein and TfR in the cell periphery calculated using Pearson's Correlation Coefficient (mean \pm SEM, EEA1 and VPS26: $n=4$; AP-2 and LAMP1: $n=3, n$ : independent experiments with 15-30 images analyzed per condition per experiment). (c) Distribution of Rab proteins in HeLa cells treated with scrambled (scr) siRNA or depleted of MTM1 (MTM1 KD). Confocal images of HeLa cells expressing eGFPRabs (Rab4, Rab5, Rab11, Rab14, Rab35). Note the strong accumulation of early and recycling endosomal Rabs on sub-plasma membrane TfR-containing endosomes in HeLa cells depleted of MTM1. Representative images from one of 3 independent experiments (Rab4, Rab5, Rab11, Rab14) or one experiment with 15 images per 
condition (Rab35) are sown. Scale bar, $10 \mu \mathrm{m}$; magnified insets: $1 \mu \mathrm{m}$. (d) Quantification of colocalization between the indicated Rab protein and TfR in the cell periphery calculated using Pearson's Correlation Coefficient (mean \pm SEM, Rab5 and Rab11: $n=4$; Rab4 and Rab14: $n=3, n$ : independent experiments with 1530 images analyzed per condition per experiment).

In contrast, TfR-cluster in MTM1-depleted cells were devoid of late endosomal/ lysosomal marker LAMP1, Rab35, a marker for fast recycling to the cell surface (Kouranti, Sachse et al. 2006), APPL1, a marker for signaling endosomes (Zoncu, Perera et al. 2009), endocytic adaptor proteins such as AP-2, retromer components involved in retrograde endosome-toGolgi transport (i.e. Vps26, (Bonifacino and Hurley 2008)) or the Golgi marker GM130 (Figure 16a-c). The negative correlation between these compartments and TfR further indicates that upon loss of MTM1 intermixing of sub-cellular compartments does not occur. Thus, defective exocytosis is not a secondary effect of a loss of membrane identity. Taken together, these data strengthen our previous conclusion that endocytosis, degradative sorting and retrograde transport remain unaltered upon loss of MTM1.
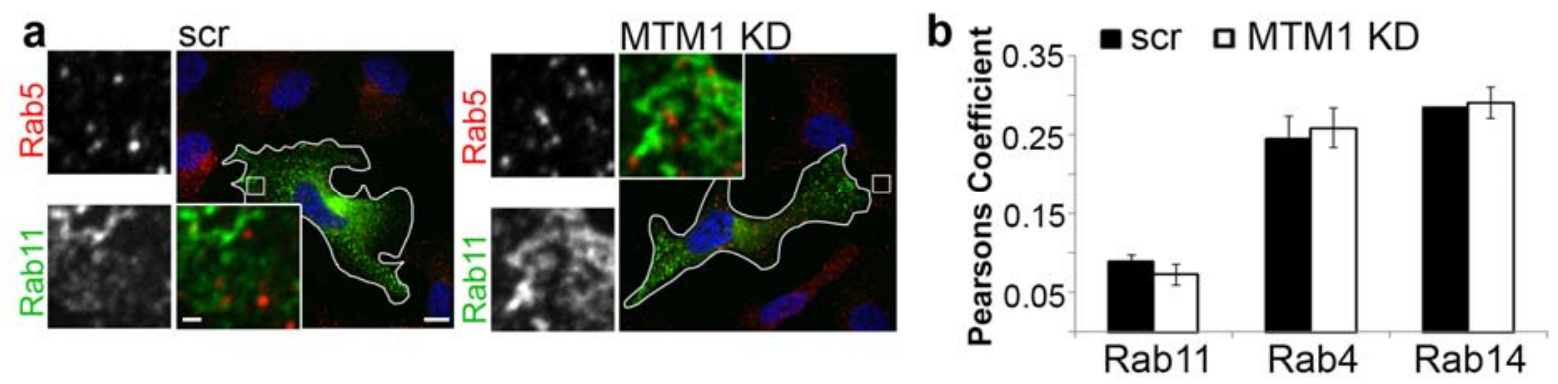

Figure 17: Distinct early and recycling endosomal compartments in MTM1-depleted cells

(a) HeLa cells treated with scrambled (scr) siRNA or depleted of MTM1 (MTM1 KD) and expressing eGFPRabs (Rab4, Rab11, Rab14). Rab5 was endogenously labeled using a Rab5-specific antibody. Representative images from one of 3 independent experiments are shown. Scale bar, $10 \mu \mathrm{m}$; magnified insets, $1 \mu \mathrm{m}$. (b) Pearson's Correlation Coefficients between eGFP-Rabs and endogenous Rab5 in the cell periphery (mean \pm SEM, $n=3$ independent experiments with 15-30 images analyzed per condition per experiment).

An important class of endosomal adaptor proteins involved in endosomal sorting are members of the SNX family (Cullen 2008). As MTM1 is a PI(3)P 3-phosphatase we addressed the subcellular localization of PI(3)P-binding SNXs with respect to their accumulation at TfR-containing endosomes. Remarkably, PI(3)P-binding SNXs implicated in endosomal sorting and with known localization to early and recycling endosomes, such as SNX1, SNX3, SNX4, SNX8, SNX17 and SNX27, accumulated on TfR clusters in MTM1depleted cells (Figure 18a, b). In contrast, TfR-cluster were negative for SNX15 (Figure 18a, b), which was previously implicated in earlier sorting events such as the progression of CCVs to PI(3)P-enriched early endosomes (Danson, Brown et al. 2013). Many SNXs contain BAR domains with membrane deforming activities (Carlton, Bujny et al. 2004), some of which we 
found enriched on TfR-containing endosomes upon MTM1 depletion. Accordingly, we frequently observed strong tubulation of the endosomal recycling compartment in MTM1 knockdown cells (Figure 18c).
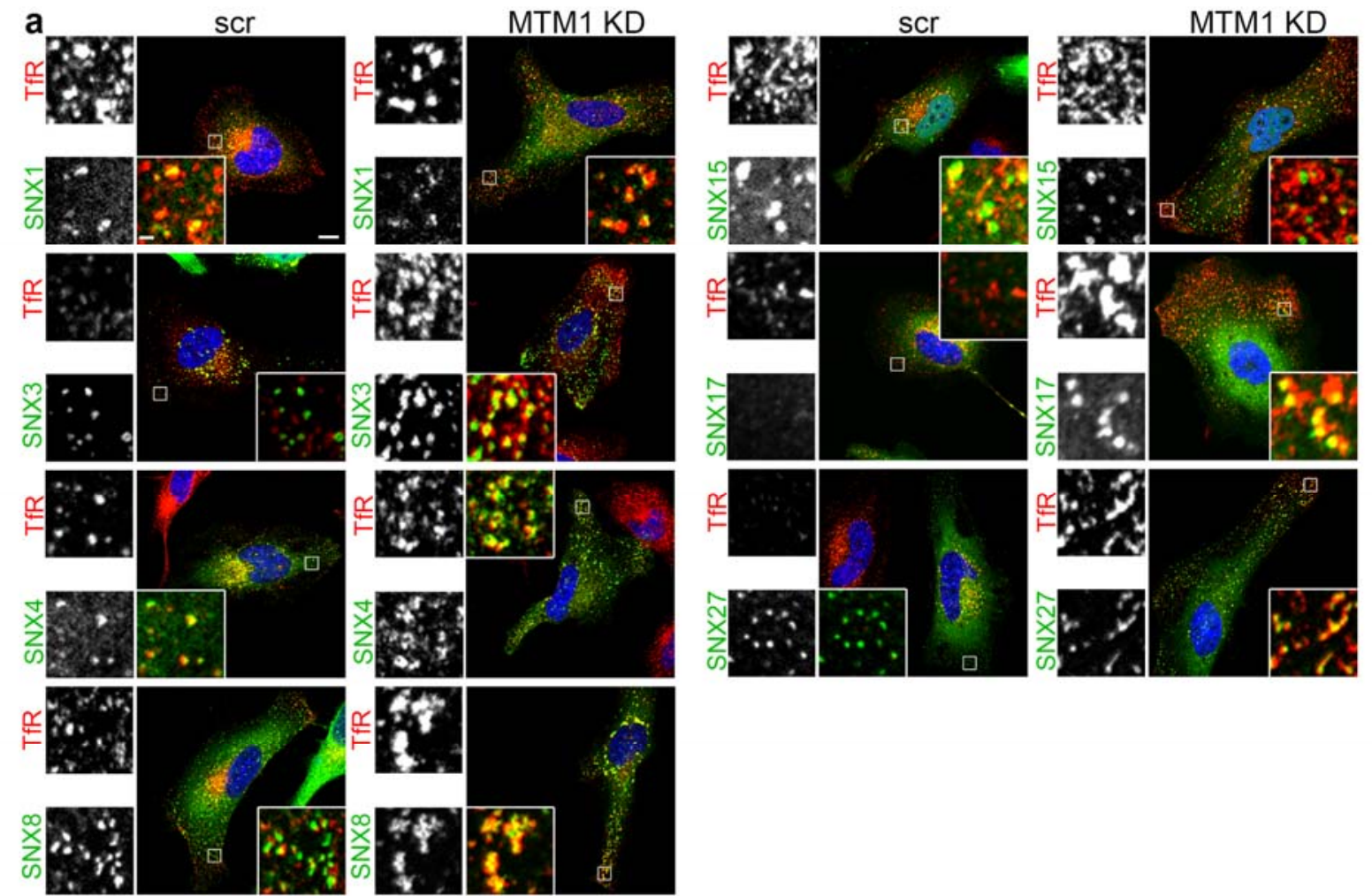

\section{b}
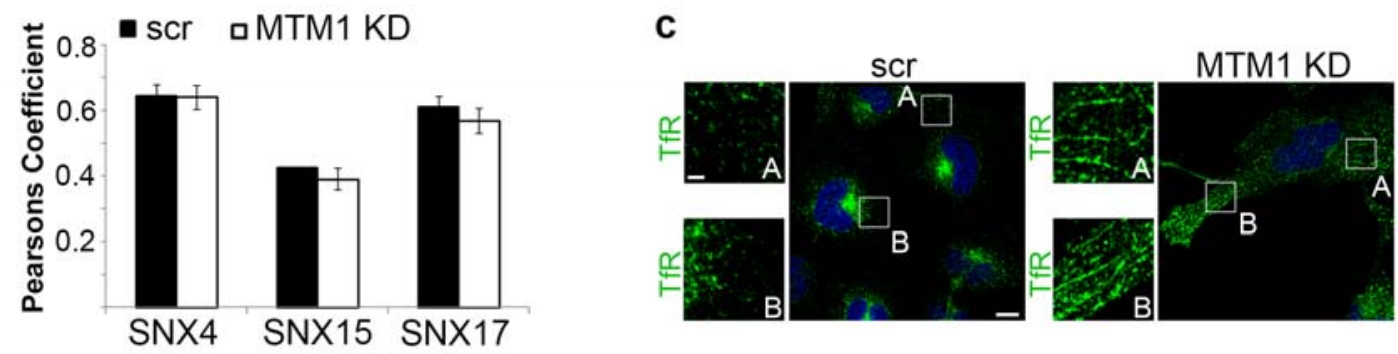

Figure 18: Sorting nexins in MTM1-depleted cells

(a) Accumulation of PI(3)P-binding SNXs (eGFP-tagged SNX1, -SNX3, -SNX4, -SNX8, SNX17, -SNX27) but not eGFP-tagged SNX15 on sub-plasma membrane TfR endosomes in HeLa cells depleted of MTM1. Representative images from one of 3 experiments (SNX4, SNX15, SNX17) or from one experiment with 6-20 images (SNX1, SNX3, SNX8, SNX27) per condition are shown. Scale bar, $10 \mu \mathrm{m}$; magnified insets, $1 \mu \mathrm{m}$. (b) Quantification of colocalization between the indicated SNX protein and TfR in the cell periphery calculated using Pearson's Correlation Coefficient (mean \pm SEM, $n=3$ independent experiments with 15-30 images analyzed per condition per experiment). (c) TfR-containing endosomal tubules accumulate in HeLa cells depleted of MTM1. Representative images from one of 3 independent experiments are shown. Scale bar, $10 \mu \mathrm{m}$; magnified insets, $2 \mu \mathrm{m}$. 

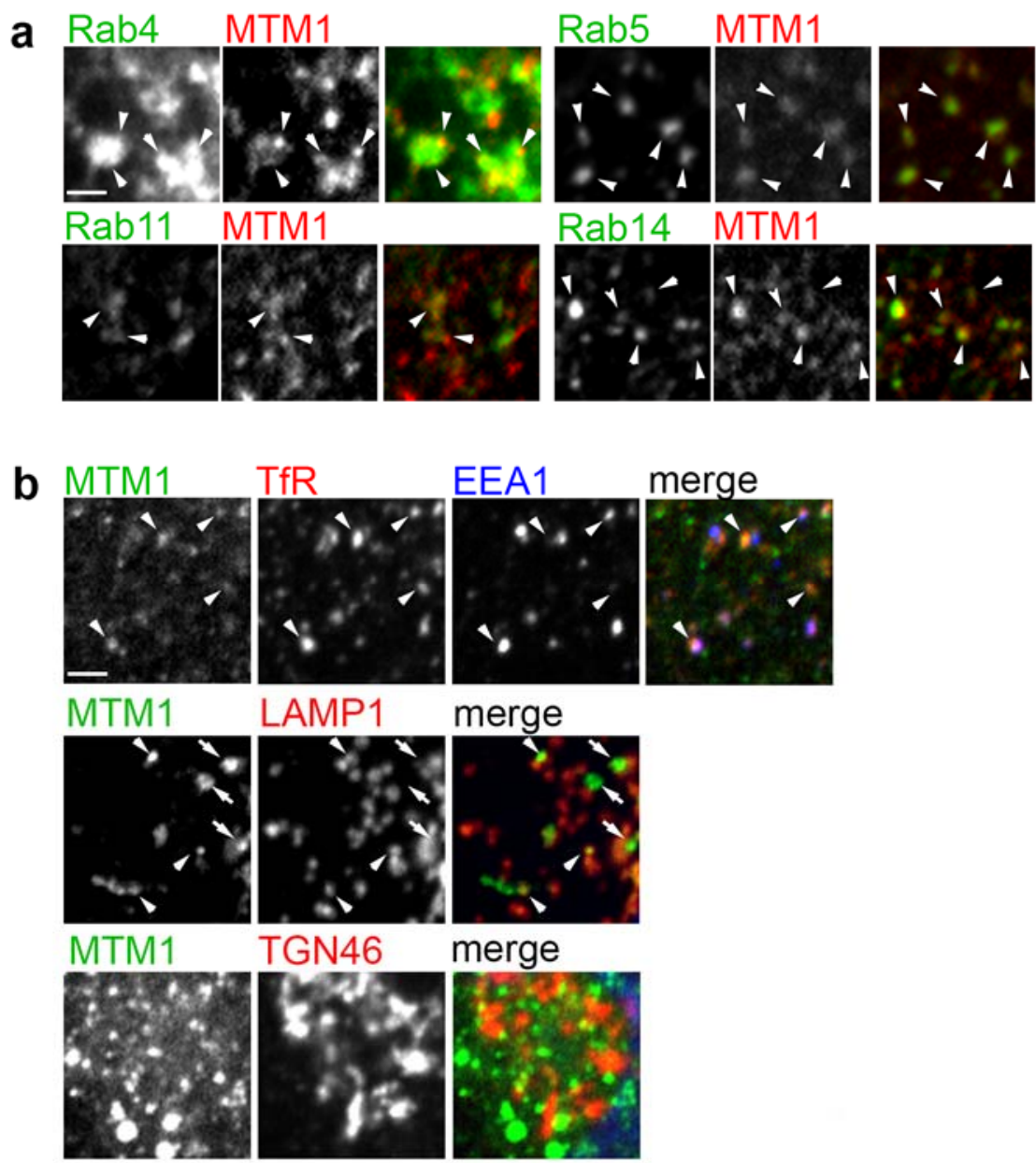

Figure 19: Endosomal localization of MTM1

(a) HeLa cells co-expressing eGFP-Rabs and mCherry-MTM1. Colocalization of MTM1 with early and recycling endosomal Rabs (Rab4, Rab5, Rab11, Rab14) is indicated by arrowheads. Representative images from one experiment with 10-20 images per condition are shown. Scale bar, $2 \mu \mathrm{m}$. (b) HeLa cells expressing eGFPMTM1 were labeled for the indicated endosomal markers by specific antibodies. Arrowheads mark colocalization of MTM1 with the indicated proteins, whereas arrows mark MTM1-positive endosomes devoid of the indicated protein. Representative images from one experiment with 10-20 images per condition are shown. Scale bar, $2 \mu \mathrm{m}$.

\subsubsection{MTM1 localizes to early and recycling endosomes}

The experiments so far focused on MTM1 function in endosomal trafficking with an emphasis on endosomal recycling and mostly comparing MTM1-depleted with control cells. The localization of the enzyme itself, on the other hand, was not addressed. Unfortunately, antibodies raised against human MTM1 did not recognize the endogenous protein in immunocytochemistry stainings. Thus, the intracellular localization of MTM1 was studied by expressing the phosphatase tagged with fluorescent proteins. mCherry-MTM1 localized predominantly to early endosomes (i.e. EEA1-, Rab4- and Rab5-positive) and recycling endosomes (i.e. Rab11- and Rab14-positive). Most importantly, MTM1 was found on TfRpositive endosomes, while colocalization with the late endosomal/ lysosomal marker LAMP1 
or the trans-Golgi-network marker TGN46 was less prominent (Cao, Laporte et al. 2007) (Figure 19). Consistent with its substrate specificity MTM1 preferentially associated with $\mathrm{PI}(3) \mathrm{P}$-containing liposomes in flotation assays in vitro while only minor binding to $\mathrm{PI}(4) \mathrm{P}$ and $\mathrm{PI}(4,5) \mathrm{P}_{2}$ - over PI-containing control liposomes could be observed (data not shown, experiments performed by Michael Krauß). The preferential localization of MTM1 to PI(3)Ppositive, TfR-containing early and recycling endosomes is in full agreement with its implication in PI(3)P-dependent exit from the endosomal system to allow surface delivery of endosomal cargo.

\subsubsection{PI(3)P accumulates on endosomes in MTM1-depleted cells}

PIs are key determinants of membrane identity and are extensively interconverted during endosomal progression. Whereas $\mathrm{PI}(4) \mathrm{P}$ and $\mathrm{PI}(4,5) \mathrm{P}_{2}$ define the $\mathrm{PM}$, secretory, Golgiderived vesicle are marked by PI(4)P. Endosomes, although they might originate from the PM by internalization, are enriched in PI 3-phosphates such as $\mathrm{PI}(3) \mathrm{P}$ and $\mathrm{PI}(3,5) \mathrm{P}_{2}$. Conversion of PIs and maintenance of compartmental identity during endosomal transport is achieved by PI-kinases and -phosphatases. Therefore, we investigated whether loss of MTM1 is accompanied by changes in the PI balance of the endosomal system.

As MTM1 is a known PI(3)P 3-phosphatase, which predominantly associated with PI(3)P-containing liposomes, we were especially interested in determining intracellular PI(3)P level. To do so, we used two different PI(3)P-probes: the recombinant, eGFP-tagged 2xFYVE domain of Hrs and the GST-tagged Phox domain of p40, chemically conjugated to Alexa488 (Munson, Allen et al. 2015). Consistent with identical PI-specificity, completely overlapping subcellular compartments were labeled by the 2xFYVE- and Phox-probes (Figure 20c). To determine the concentration at which the eGFP-2xFYVE probe detects $\mathrm{PI}(3) \mathrm{P}$ at a linear range, we titrated domain in the detection reaction (Figure 20a). Additionally, we controlled for binding specificity by applying the broad-range PI3K-inhibitor wortmannin. Wortmannin abolished FYVE-domain staining completely (Figure 20b; as has been shown previously for the GST-Phox-Alexa488 probe (Munson, Allen et al. 2015)). Expression of wild-type MTM1 but not mutant phosphatase-inactive (C375S) MTM1 significantly reduced cellular PI(3)P levels (Figure 20d). These data clearly confirm that the phosphatase activity of MTM1 is directed towards $\mathrm{PI}(3) \mathrm{P}$, but also prove that the C375S mutation completely abolishes phosphatase activity and that the 2xFYVE- and Phox-probes specifically detect PI(3)P.

Loss of MTM1 resulted in a specific increase in PI(3)P level, while other PI species, i.e. $\mathrm{PI}(4) \mathrm{P}, \mathrm{PI}(3,4) \mathrm{P}_{2}$ and $\mathrm{PI}(4,5) \mathrm{P}_{2}$, remained unaffected (Figure $\left.21 \mathrm{a}\right)$. These data are 
consistent with previously published biochemical measurements of PI level in MTM1depleted cells using $\left[\mathrm{H}^{3}\right]$-inositol labeling (Cao, Backer et al. 2008). Furthermore, sub-plasma membrane TfR-endosomes were specifically enriched in PI(3)P upon loss of MTM1 (Figure 21a), matching the prominent increase in PI(3)P-effector proteins (see Figure 17, i.e. EEA1 and Figure 18, i.e. sorting nexins such as SNX1, SNX3, SNX4, SNX17).
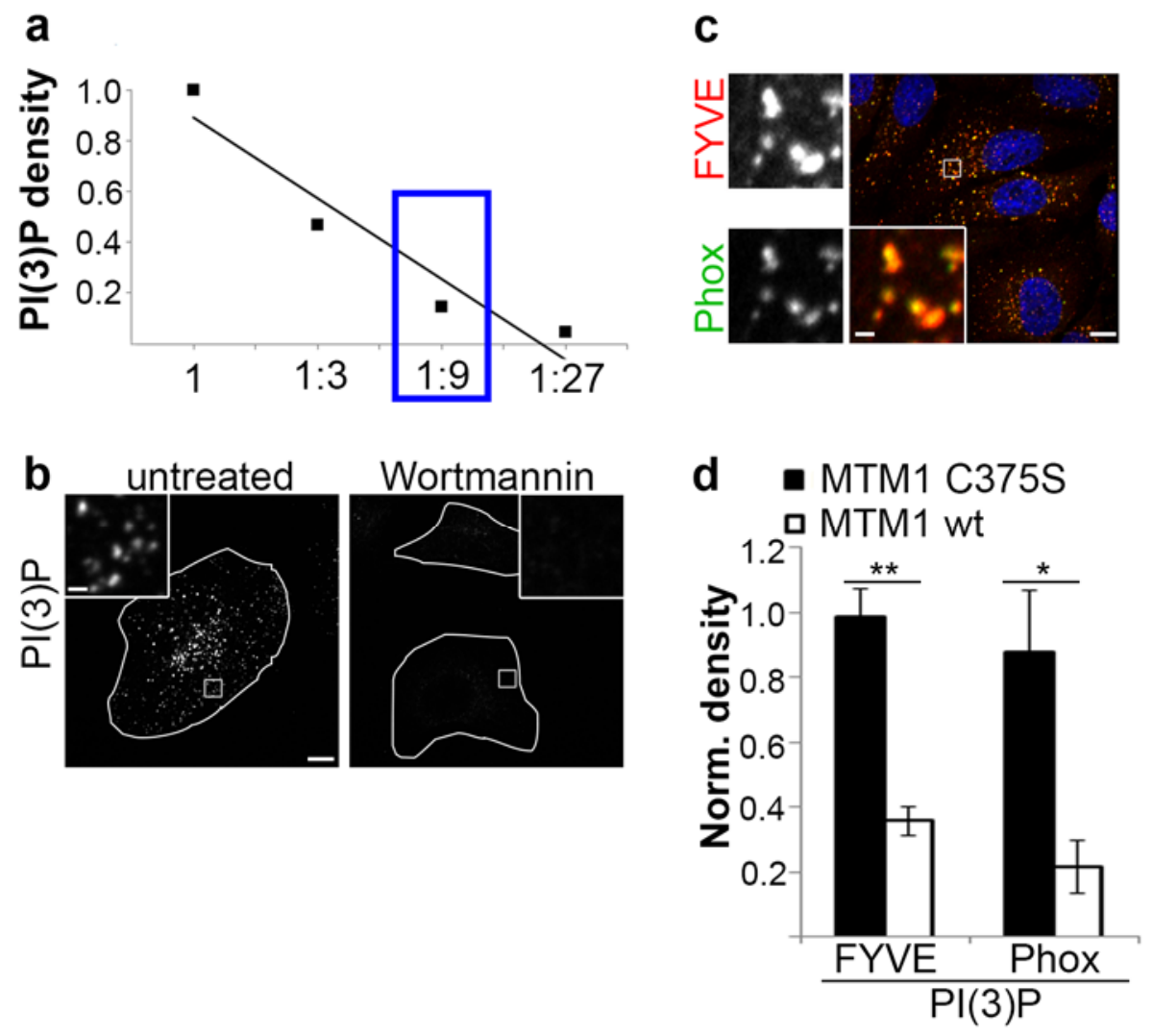

Figure 20: Semi-quantitative measurement of PI(3)P using recombinant PI(3)P-binding domains

(a) HeLa cells were labeled for PI(3)P using different eGFP-2xFYVE domain concentrations, starting with 2.25 $\mu \mathrm{g} / \mathrm{ml}$ (1) and subsequent 1:3 dilutions of (1) $(0.75 \mu \mathrm{g} / \mathrm{ml}(1: 3), 0.25 \mu \mathrm{g} / \mathrm{ml}(1: 9), 0.083$ (1:27)). PI(3)P levels per cell area were quantified. The titration curve is shown and the linear range indicated by the blue box. (b) HeLa cells treated with DMSO or $2 \mu \mathrm{M}$ wortmannin were stained for PI(3)P using purified eGFP-2xFYVE. Note the absence of eGFP-2xFYVE staining in cells treated with the broad-spectrum PI 3-kinase inhibitor wortmannin. Representative images from one experiment with 10-15 images per condition are shown. Scale bar, $10 \mu \mathrm{m}$; magnified insets, $1 \mu \mathrm{m}$. (c) HeLa cells were labeled for PI(3)P using eGFP-2xFYVE domain and Phox-Alexa488 as indicated. Representative images from one experiment with 10-15 images per condition are shown. Scale bar, $10 \mu \mathrm{m}$; magnified insets: $1 \mu \mathrm{m}$. (d) Decreased PI(3)P levels in HeLa cells overexpressing wild-type (wt) but not mutant inactive (C375S) mCherry-MTM1. Depletion of PI(3)P is consistent with PI(3)P 3-phosphatase activity of MTM1. Cells were labeled for PI(3)P using eGFP-2xFYVE domain or Phox-Alexa488 as indicated and the relative levels of $\mathrm{PI}(3) \mathrm{P}$ normalized to cell area were quantified (mean $\pm \mathrm{SEM}, n=3$ independent experiments with 15-30 images analyzed per condition per experiment, ${ }^{*} P<0.05, * * P<0.01$, unpaired, two-tailed $t$-test).

As antibody labeling critically depends on accessibility of the antigen, we hypothesized that removal of PI(3)P-effector proteins should unmask an additional PI(3)P pool, which was not detectable in MTM1-depleted cells. As indicated above, the accumulation of sorting nexins might account for the tubular shape of defective recycling 
endosomes in MTM1-KD cells (see Figure 18). We therefore depleted candidate SNXs together with MTM1 to see if this would result in a further increase of cellular PI(3)P levels.

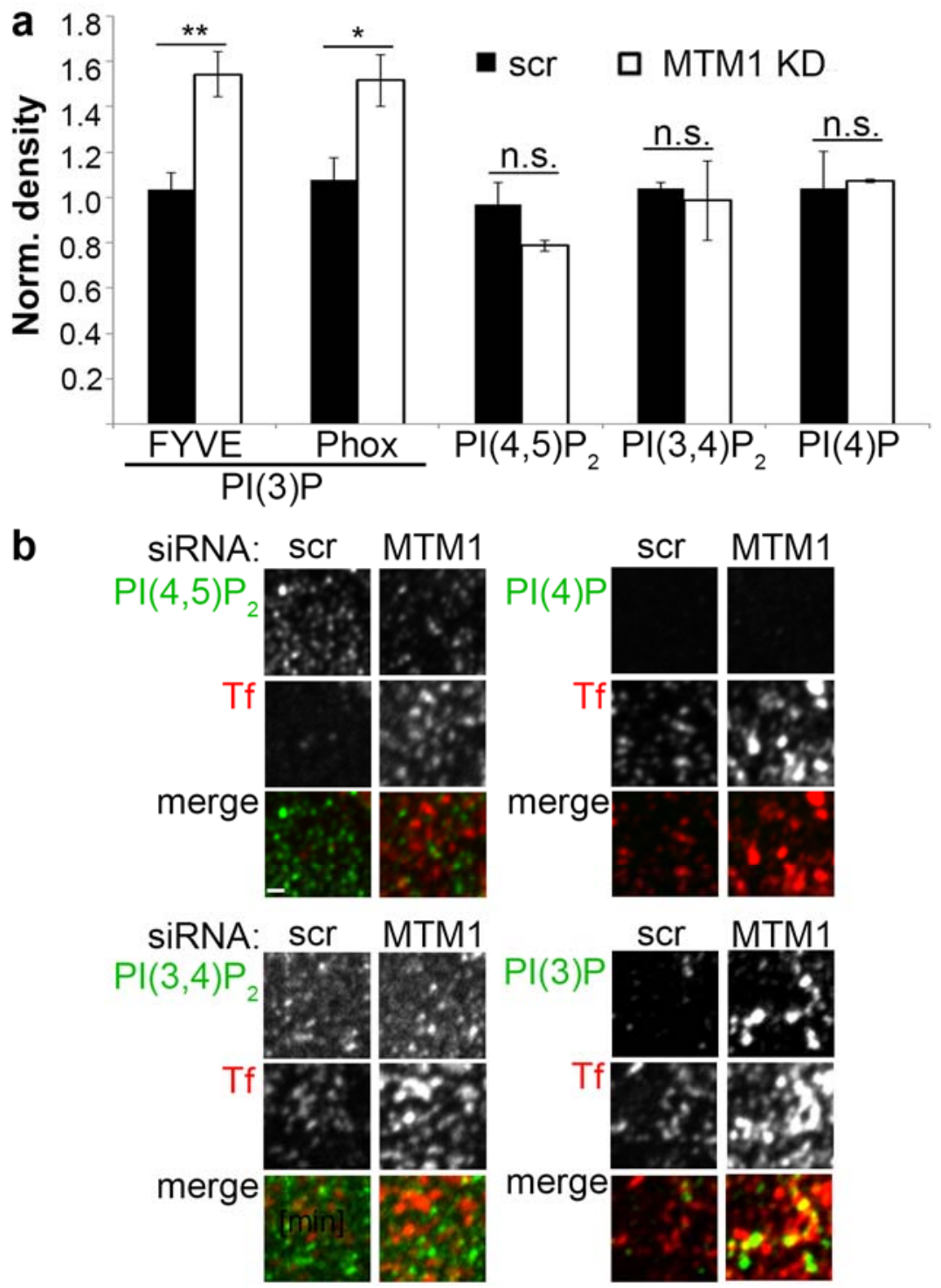

Figure 21: Selective increase in total PI(3)P levels and PI(3)P accumulation at TfR-cluster upon MTM1 depletion

(a) Selective increase of PI(3)P in MTM1-depleted HeLa cells. Shown are the relative levels of PI(3)P, labeled using purified GFP-2xFYVE or GST-Phox domain, $\mathrm{PI}(4) \mathrm{P}, \mathrm{PI}(3,4) \mathrm{P}_{2}$ and $\mathrm{PI}(4,5) \mathrm{P}_{2}$, labeled by PIPx-specific antibodies, normalized to cell area (mean $\pm \mathrm{SEM}, n=3$ independent experiments with 15-30 images analyzed per condition per experiments, ${ }^{*} P<0.05,{ }^{* *} P<0.01$, unpaired, two-tailed $t$-test). (b) PIPx stainings in MTM1depleted HeLa cells: eGFP-2xFYVE was used to detect PI(3)P, PI(4)P, PI(3,4) $\mathrm{P}_{2}$ and $\mathrm{PI}(4,5) \mathrm{P}_{2}$ were labeled with specific antibodies. Sub-plasma membrane TfR accumulations are selectively enriched in $\mathrm{PI}(3) \mathrm{P}$. Representative images from one of 3 independent experiments are shown. Scale bar, $1 \mu \mathrm{m}$.

SNX4, previously shown to be involved in TfR-sorting and recycling (Traer, Rutherford et al. 2007), as well as SNX17, implicated in recycling of endosomal cargo containing a NPxY-motif within the cytoplasmic tail such as $\beta 1$-integrin (Bottcher, Stremmel et al. 2012; Steinberg, Heesom et al. 2012) and TfR, were the most promising PI(3)P-effector 
proteins to use for co-depletion experiments. While single knockdown of either SNX4 or SNX17 had no effect on total PI(3)P levels as compared to scrambled-treated controls, codepletion of MTM1 and SNX4 or SNX17 significantly increased the detectable PI(3)P pool compared to single knockdowns, including MTM1 (Figure 22). Taken together, these data clearly show that enrichment of PI(3)P on TfR-endosomes causes an accumulation of PI(3)Peffector proteins, and suggest that this results in defective exit from endosomes en route to the plasma membrane. Based on these results we further hypothesized that MTM1-mediated dephosphorylation of $\mathrm{PI}(3) \mathrm{P}$ is required to remove these $\mathrm{PI}(3) \mathrm{P}$-effector protein to allow fusion with the plasma membrane.
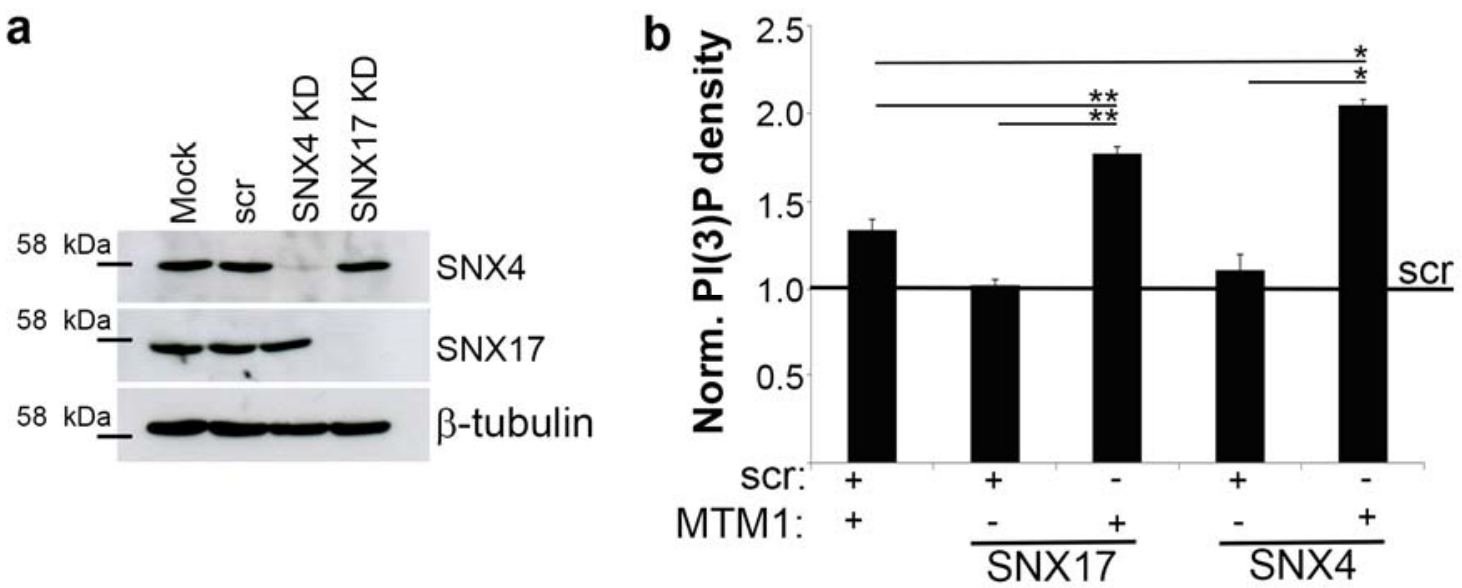

Figure 22: Removal of PI(3)P-binding effector proteins unmasked a previously not detectable pool of PI(3)P in MTM1-depleted cells

(a) Efficient silencing of SNX4 and SNX17 in HeLa cells as demonstrated by immunoblotting. (b) Co-depletion of MTM1 and SNX4 or SNX17 reveals additional pools of PI(3)P inaccessible in cells only depleted of MTM1. Relative levels of PI(3)P in cells treated with the indicated siRNAs (mean \pm SEM, $n=3$ independent experiments with 15-30 images analyzed per condition per experiment, $* P<0.05, * * P<0.01$, unpaired, twotailed $t$-test).

\subsubsection{PI(3)P levels can be manipulated by genetic and pharmacological tools}

In order to test whether MTM1-mediated removal of endosomal PI(3)P is a prerequisite for the dissociation of PI(3)P-effector proteins, and thus for successful endosomal recycling, we next investigated the effects of manipulating endosomal PI(3)P levels, synthesis, turn-over or localization in MTM1-depleted cells (Idevall-Hagren and De Camilli 2015).

Vps34 is the major endosomal PI 3-kinase in humans (Raiborg, Schink et al. 2013). Reduction of endosomal PI(3)P synthesis by depletion of Vps34 in MTM1 knockdown cells largely restored perinuclear TfR localization, caused by loss of MTM1 (Figure 23a, b). 
a

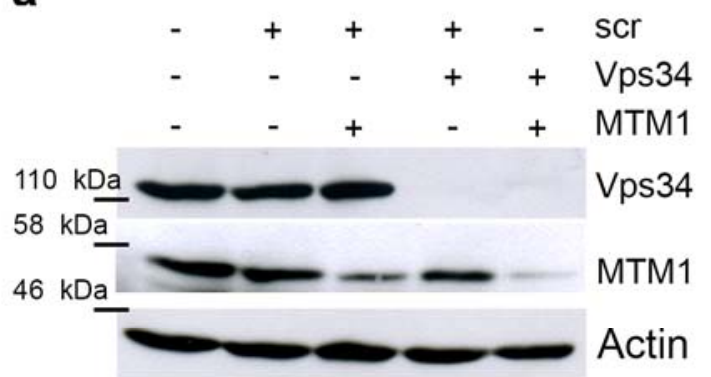

b

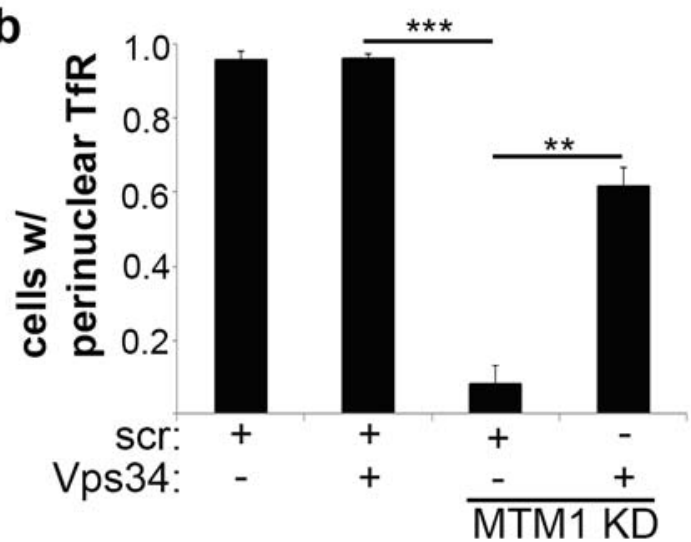

C

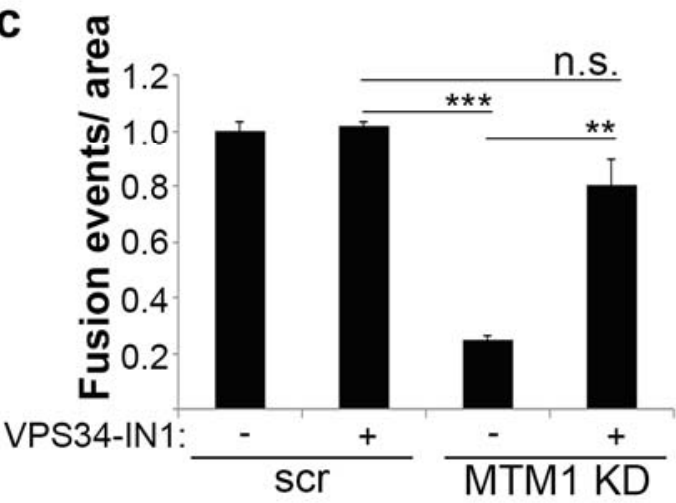

Figure 23: Reduction of PI(3)P synthesis counter-acts MTM1 loss-of-function defects

(a) Efficient co-depletion of Vps34 and MTM1 in HeLa cells as demonstrated by immunoblotting. (b) Codepletion of MTM1 and Vps34 restores perinuclear TfR localization. Normalized fraction of HeLa cells with perinuclear TfR in cells treated with the indicated siRNAs (mean $\pm \mathrm{SEM}, n=3$ independent experiments with 15-30 images analyzed per condition per experiment, ${ }^{* *} P<0.01,{ }^{* * *} P<0.001$, unpaired, two-tailed $t$-test). (c) Pharmacological inhibition of $\mathrm{Vps} 34$ (for $1 \mathrm{~h}, 37^{\circ} \mathrm{C}$ ) rescues defective Tf exocytosis in MTM1 depleted cells. Quantified are $\mathrm{Tf}$ exocytic events normalized to cell area (mean $\pm \mathrm{SEM}, n=3$ independent experiments with 5 videos analyzed per condition per experiment, ${ }^{* *} P<0.01,{ }^{* * *} P<0.001$, unpaired, two-tailed $t$-test).
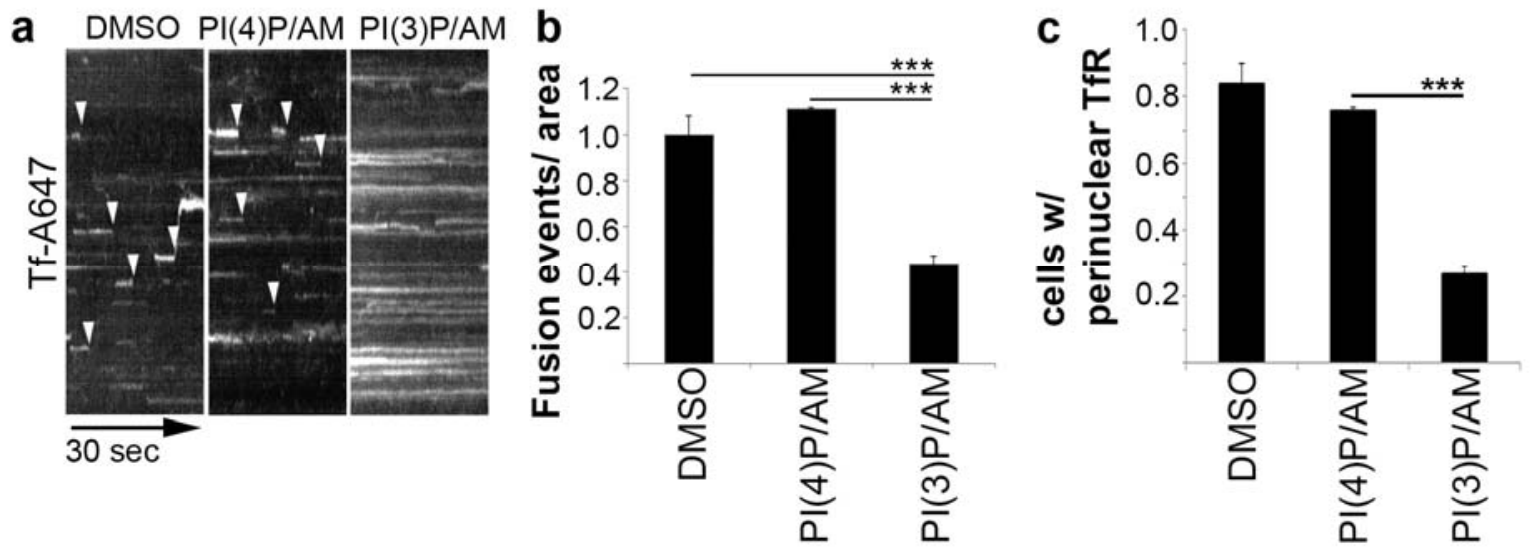

Figure 24: Exogenous oversupply of PI(3)P phenocopies MTM1 depletion

(a, b) HeLa cells were incubated with DMSO, PI(3)P/AM or PI(4)P/AM and Tf exocytosis was monitored by TIRF microscopy. (a) Kymographs of Tf fluorescence signal over $28 \mu \mathrm{m}$, arrowheads indicate time of plasma membrane fusion. (b) Quantified are Tf exocytic events/ area (mean \pm SEM, $n=3$ independent experiments with 
5 videos analyzed per condition per experiment, ${ }^{* * *} P<0.001$, unpaired, two-tailed $t$-test). (c) Exogenous oversupply of PI(3)P causes TfR misslocalization. Normalized fraction of cells with perinuclear TfR was quantified (mean $\pm \mathrm{SEM}, n=4$ independent experiments with 15-30 images analyzed per condition per experiment, ${ }^{* * *} P<0.001$, unpaired,two-tailed $t$-test).

Furthermore, acute inhibition of $\mathrm{PI}(3) \mathrm{P}$ synthesis by applying the Vps34-specific inhibitor VPS34-IN1 (Bago, Malik et al. 2014) rescued defective Tf-exocytosis in MTM1-depleted cells (Figure 23c).

On the other hand, elevation of intracellular PI(3)P but not PI(4)P levels by exogenous addition of cell permeable PIP-analogues (Subramanian, Laketa et al. 2010) mimicked MTM1 loss-of function with respect to defective Tf exocytosis and TfR mislocalization (Figure 24).

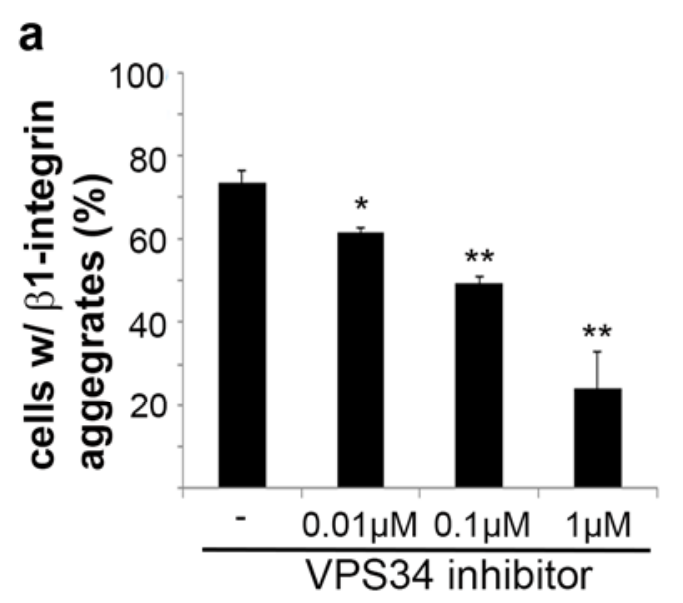

b

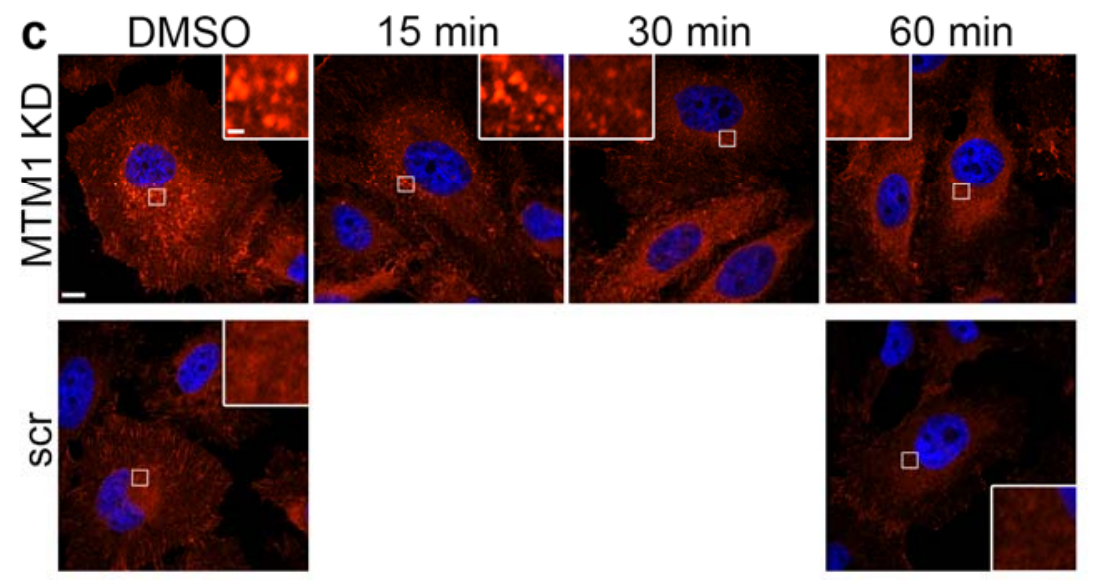

Figure 25: Pharmacological inhibition of Vps34 rescues $\beta 1$-integrin accumulation in XLCNM patient and MTM1-depleted HeLa cells

(a) XLCNM patient cells were treated with the indicated concentrations of VPS34-IN1. Graph depicts percentage of cells with aggregated $\beta 1$-integrin (mean \pm SEM, $n=3$ independent experiments with 15-30 images analyzed per condition per experiment, ${ }^{*} P<0.05,{ }^{* *} P<0.01$, unpaired, two-tailed $t$-test) and (b) immunocytochemistry stainings with $\beta 1$-integrin antibody. Representative images from one of 3 independent experiments are shown. Scale bar, $10 \mu \mathrm{m}$; magnified insets: $1 \mu \mathrm{m}$. (c) HeLa cells treated with scrambled siRNA or depleted of MTM1 were treated with VPS34-IN1 for the indicated times. Pharmacological inhibition of Vps34 rescues $\beta 1$-integrin accumulation in MTM1-depleted HeLa cells within $60 \mathrm{~min}$, assessed by $\beta 1$-integrinspecific antibody labeling. Representative images from one experiment with 10-15 images per condition per experiment are shown. Scale bar, $10 \mu \mathrm{m}$; magnified insets: $1 \mu \mathrm{m}$. 

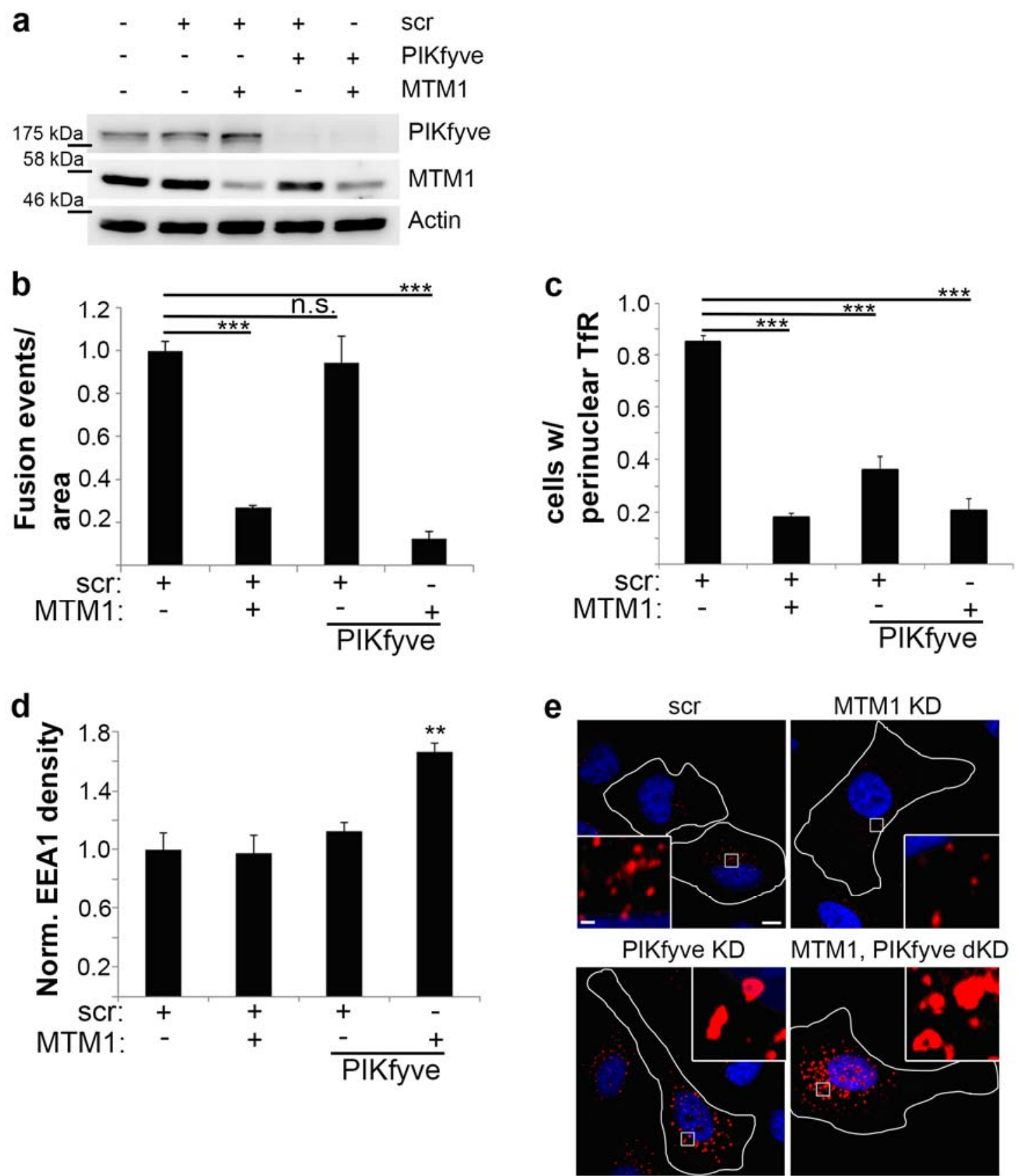

Figure 26: Manipulation of PI(3,5) $P_{2}$ - manipulation by PIKfyve depletion

(a) Efficient co-depletion of PIKfyve and MTM1 in HeLa cells as demonstrated by immunoblotting. (b) Codepletion of MTM1 and PIKfyve does not restore defective Tf exocytosis in MTM1-depleted cells (Tf exocytic events/ area; mean \pm SEM, $n=3$ independent experiments with 5 videos analyzed per condition per experiment, *** $P<0.001$, unpaired, two-tailed $t$-test). (c) Co-depletion of PIKfyve and MTM1 is unable to restore perinuclear TfR localization. Normalized fraction of cells with perinuclear TfR in cells treated with the indicated siRNAs (mean \pm SEM, $n=3$ independent experiments with 15-30 images analyzed per condition per experiment, $* * * P<0.001$, unpaired, two-tailed $t$-test). (d) Co-depletion of PIKfyve and MTM1 leads to increased EEA1 density (mean \pm SEM, $n=3$ independent experiments with 15-30 images analyzed per condition per experiment, $* * P<0.01$, unpaired, two-tailed $t$-test). (e) Swelling of EEA1-positive endosomes in PIKfyvedepleted and MTM1/ PIKfyve co-depleted cells. Representative images from one of 3 independent experiments are shown. Scale bar, $10 \mu \mathrm{m}$; magnified insets, $1 \mu \mathrm{m}$.

Importantly, $\beta 1$-integrin accumulations in MTM1-depleted HeLa cells and XLCNM patients were cleared in a concentration- and time-dependent manner by VPS34-IN1 treatment (Figure 25). This data strengthens the strong correlation between elevated PI(3)P 
level, defective exocytosis and loss-of-function mutations of MTM1 in XLCNM patients. VPS34-IN1 could thus be suitable for the pharmacological treatment of XLCNM.

MTM1 can accept $\mathrm{PI}(3,5) \mathrm{P}_{2}$ in addition to $\mathrm{PI}(3) \mathrm{P}$ as a substrate. As there are no $\mathrm{PI}(3,5) \mathrm{P}_{2}$ specific antibodies or -binding probes available, we choose a genetic approach to test how manipulation of $\mathrm{PI}(3,5) \mathrm{P}_{2}$ influences phenotypic changes in MTM1 depleted cells. En route to late endosomes $\mathrm{PI}(3) \mathrm{P}$ can be converted to $\mathrm{PI}(3,5) \mathrm{P}_{2}$ by the $\mathrm{PI}(3) \mathrm{P}$ 5-kinase PIKfyve, thus consuming endosomal PI(3)P and concomitantly elevating $\mathrm{PI}(3,5) \mathrm{P}_{2}$ (Ikonomov, Sbrissa et al. 2006). Knocking down PIKfyve in MTM1-depleted cells did neither rescue Tf-exocytic defects nor TfR-mis-localization, that have been observed upon loss of MTM1(Figure 26a-c). Co-depletion rather aggravated the phenotype, as indicated by the swelling of the early endosomal compartment (Figure 26d, e). We therefore hypothesized that blocking PI(3)P turnover by depleting PIKfyve in MTM1-KD cells further increases PI(3)P level, causing excessive endosome fusion. Moreover, PIKfyve depletion mimicked the peripheral accumulation of TfR-endosomes, although less prominently than the loss of MTM1, while exocytosis was not affected (Figure 26a-c). Thus, we conclude that exit from endosomes en route to late endosomes/ lysosomes functions as a save guard in MTM1-depleted cells to avoid accumulation of early endosomal PI(3)P. Despite the fact that $\mathrm{PI}(3,5) \mathrm{P}_{2}$ could be an additional substrate for MTM1 3-phosphatase activity in vitro (Cao, Backer et al. 2008; Hnia, Tronchere et al. 2011), lowering endosomal PI(3,5) $\mathrm{P}_{2}$ levels by co-depletion of PIKfyve did not affect phenotypic changes observed upon loss of MTM1. Thus, endosomal defects caused by loss of MTM1 are primarily due to elevated PI(3)P, but not $\mathrm{PI}(3,5) \mathrm{P}_{2}$ levels.

The peripheral accumulation of endosomes in cells lacking active MTM1 could be caused by (1) defective inward, minus-end directed transport of sorting endosomes to the perinuclear compartment or (2) accumulation of fusion-deficient endosomes underneath the plasma membrane after successive progression through the endosomal system - inward, followed by plus-end directed outward transport -.

Loss of Kif16b, a plus-end directed, PI(3)P-dependent kinesin (Hoepfner, Severin et al. 2005), abolished outward transport and resulted in a more concentrated perinuclear localization of TfR without affecting exocytosis. Consistent with (2), depletion of Kif16b together with MTM1 restored perinuclear TfR localization, while it could not rescue the exocytic defect (Figure 27). These data indicate that accumulation of PI(3)P upon loss of MTM1 drives PI(3)P-dependent, plus-end directed transport towards the cell periphery. Furthermore, defective exocytosis is not a secondary effect of TfR mis-localization. 
Taken together these observations establish that removal of PI(3)P by MTM1 is a prerequisite for endosomal exocytosis.
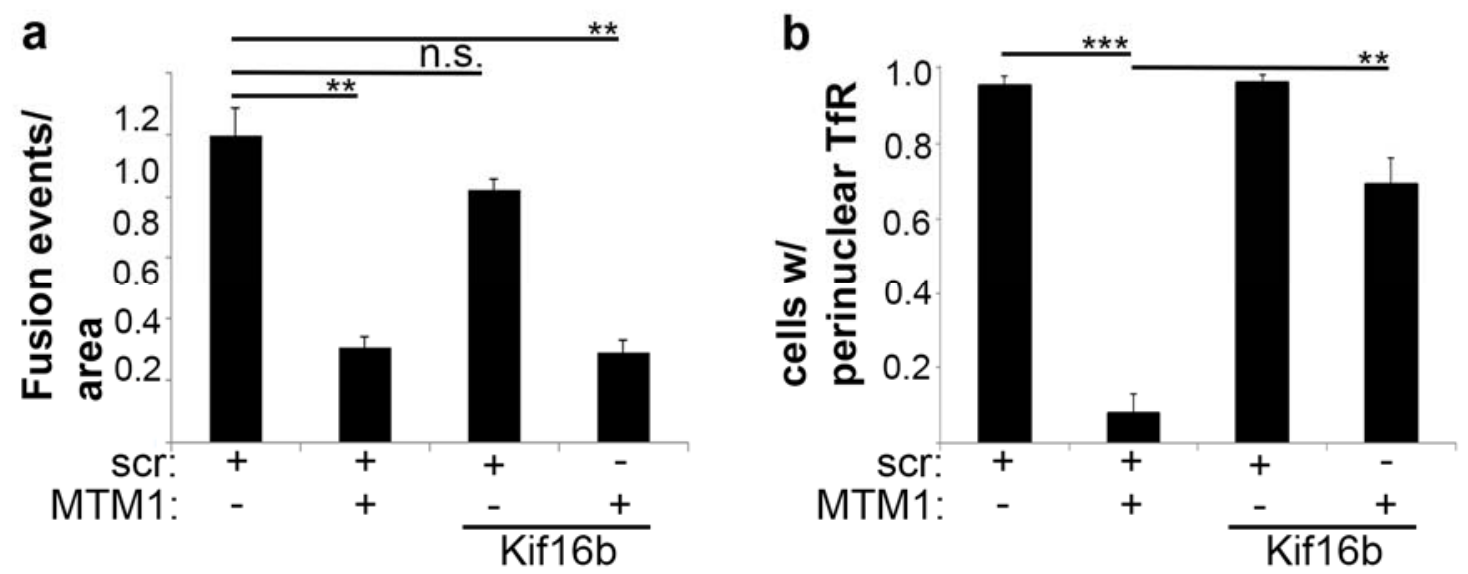

Figure 27: PI(3)P-dependent outward trafficking of endosomes depends on Kif16b

(a) Co-depletion of MTM1 and Kif16b does not restore defective Tf exocytosis in MTM1-depleted cells (Tf exocytic events/ area; mean $\pm \mathrm{SEM}, n=3$ independent experiments with 5 videos analyzed per condition per experiment, $* * P<0.01$, unpaired, two-tailed $t$-test). (b) Co-depletion of MTM1 and Kif16b restores perinuclear TfR localization. Normalized fraction of HeLa cells with perinuclear TfR in cells treated with the indicated siRNAs (mean \pm SEM, $n=3$ independent experiments with 15-30 images analyzed per condition per experiment, ${ }^{*} P<0.01, * * * P<0.001$, unpaired, two-tailed $t$-test).

\subsection{PI(3)P-to-PI(4)P conversion is required for exocyst-dependent endosomal exocytosis}

Our data so far indicate that the accumulation of endosomal PI(3)P caused by MTM1 loss of function causes a defect in exocytosis, but not in major membrane trafficking events. As dephosphorylation of $\mathrm{PI}(3) \mathrm{P}$ is required for exocytic membrane trafficking of endosomal cargo and subsequent fusion with the plasma membrane, we wanted to address whether $\mathrm{PI}(3) \mathrm{P}$ removal is also sufficient. As the plasma membrane is predominantly enriched in $\mathrm{PI}(4) \mathrm{P}$ and $\mathrm{PI}(4,5) \mathrm{P}_{2}$, we speculated that endosomal membranes have to acquire either one of these PI species to become fusion competent.

\subsubsection{Exocytic vesicles acquire PI(4)P before plasma membrane fusion}

To address PI content of exocytic vesicles prior to fusion with the plasma membrane, we monitored $\mathrm{Tf}$ exocytosis in cells expressing fluorescent PI-binding probes by dual color TIRF microscopy. PI(3)P-enriched endosomes, marked by the 2xFYVE probe were unable to undergo exocytosis, while PI(3)P-negative endosomes, devoid of the 2xFYVE probe 
frequently fused with the plasma membrane (Figure 28a). Notably, these FYVE-domain enriched endosomes were stalled and immobile. Interestingly, endosomes frequently acquired PI(4)P (as detected by the 2xPH domain of FAPP1) prior to fusion and lost PI(4)P together with Tf ligand simultaneously upon exocytosis. $\mathrm{PI}(3,4) \mathrm{P}_{2}, \mathrm{PI}(4,5) \mathrm{P}_{2}$ and $\mathrm{PI}(3,4,5) \mathrm{P}_{3}$ were not detectable on exocytic vesicles (Figure 28). 2xPH-FAPP1 localization to mobile vesicles was highly dependent on its expression levels. While the majority of the FAPP1-probe localized to the Golgi-complex, we could visualize a vesicular FAPP1-pool when its expression levels were sufficiently high. Furthermore, not all endosomes fusing with the PM were decorated by the FAPP1-probe, presumably reflecting the fact that low levels of PI(4)P on endosomes were insufficient to be monitored. These data suggest that surface delivery of endosomal cargo requires a PI conversion step from PI(3)P to PI(4)P.

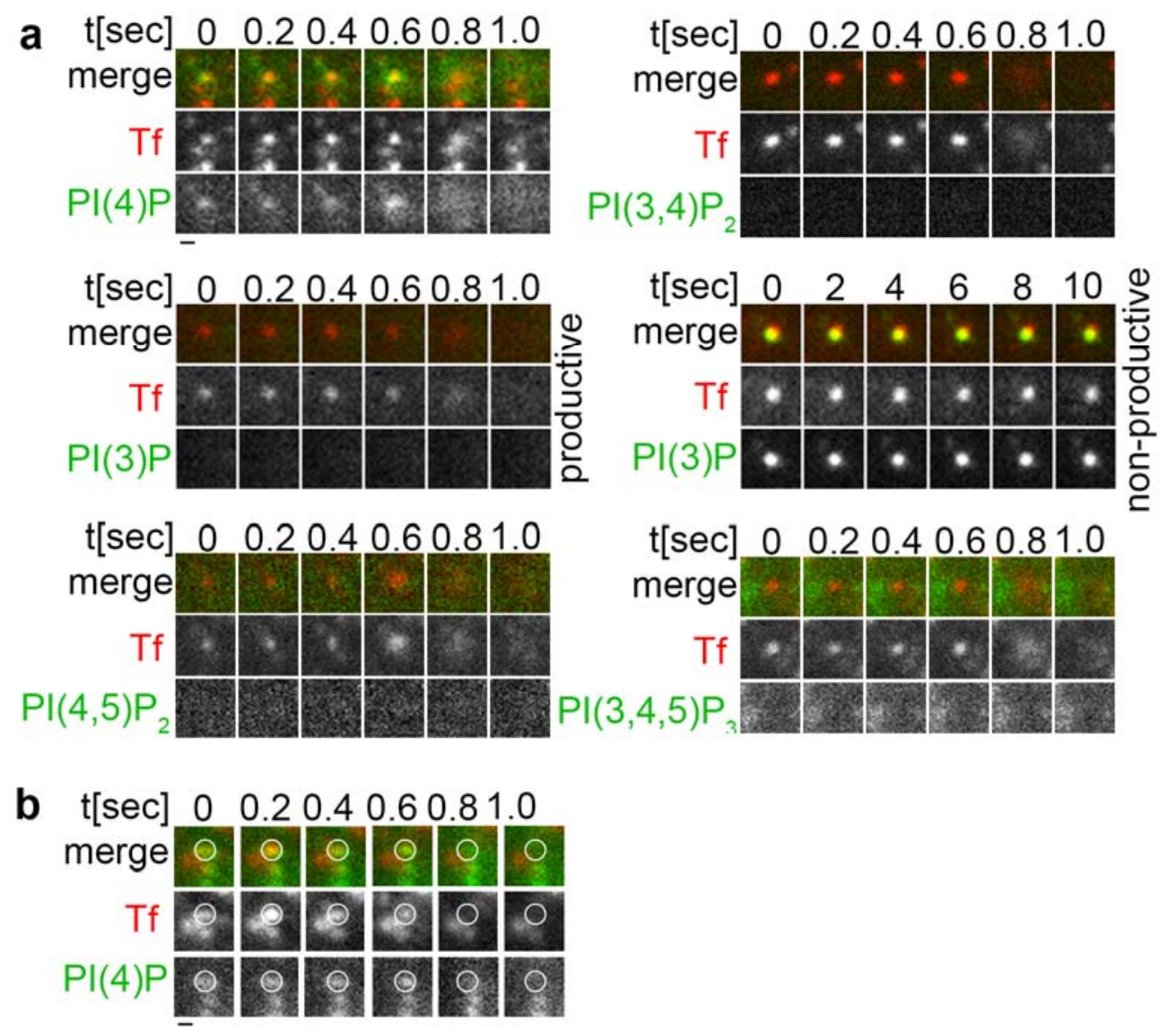

Figure 28: Exocytic vesicle acquire PI(4)P prior to plasma membrane fusion

(a) PI content of $\mathrm{Tf}$ exocytic vesicles monitored by dual-color TIRF microscopy in HeLa cells expressing the corresponding PI binding domains. Time course of representative Tf-containing endosomes is shown. Representative images from one of 4 independent experiments are shown. Scale bar: 400 nm. (b) Tf-Alexa647 (red) exocytosis from endosomes in HeLa cells overexpressing the PI(4)P reporter eGFP-2xPH-FAPP1 (green) analyzed by dual-color TIRF microscopy. Order of acquisition was reversed compared to (a) to exclude imaging artefacts. The $647 \mathrm{~nm}$ channel was imaged before acquisition of the $488 \mathrm{~nm}$ channel. Representative images from one of 2 independent experiments are shown. Scale bar: $400 \mathrm{~nm}$. 
A candidate enzyme for PI(4)P synthesis on exocytic vesicles is PI 4-kinase type II $\alpha$ (PI4K2 $\alpha$ ), a member of the PI4K family previously shown to be associated with endosomes (Minogue, Waugh et al. 2006; Mossinger, Wieffer et al. 2012), secretory vesicles (Guo, Wenk et al. 2003) and the TGN (Wang, Wang et al. 2003). PI4K2 $\alpha$ was indeed found on MTM1positive vesicles by immunocytochemistry (Figure 29) and associated with MTM1 in pull down assay (experiments were done in collaboration with Jocelyn Laporte and Anne-Sophie Nicot, data not shown). Thus, PI4K2 $\alpha$ would be an ideal candidate to generate PI(4)P on exocytic vesicles.

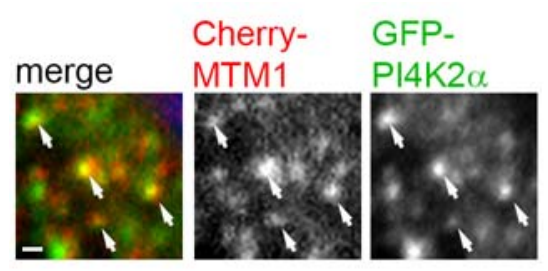

Figure 29: MTM1 and PI4K2 $\alpha$ associate on endomembranes

Colocalization of mCherry-MTM1 and eGFP-PI4K2 $\alpha$ in HeLa cells on endosomes (arrows). Representative images from one of 4 independent experiments are shown. Scale bar: $1 \mu \mathrm{m}$.

\subsubsection{PI4K2 $\alpha$ and exocyst associate and are required for exocytosis}

To clarify a potential role of PI4K $2 \alpha$ in endosomal exocytosis, we further investigated novel binding partners of PI4K2 $\alpha$. By quantitative mass spectrometry using GST-PI4K2 $\alpha$ as a bait (Mössinger, dissertation), we identified 6 out of 8 subunits of the exocyst complex (Figure 30a; Dr. Julia Mössinger, dissertation FU Berlin, 2012; (Mossinger, Wieffer et al. 2012)). Previously, the exocyst complex was shown to be required for PI(4)P-regulated secretion from the Golgi complex (Mizuno-Yamasaki, Medkova et al. 2010), polarized membrane growth (Hertzog and Chavrier 2011), Rab11-dependent exocytosis (Takahashi, Kubo et al. 2012) and tight-junction formation in yeast and mammals. Reciprocal binding between PI4K2 $\alpha$ and exocyst was confirmed by affinity chromatography (experiments were done in collaboration with Dr. Michael Krauß, data not shown) and co-immunoprecipitation (Figure 30c). Consistent with complex formation between PI4K2 $\alpha$ and exocyst, they colocalized on a subpopulation of endosomes in the cell periphery (Figure 30b). Additionally, the exocyst subunit Sec6 was identified as a MTM1 interactor in yeast-two-hybrid screening (experiments were done in collaboration with Jocelyn Laporte and Anne-Sophie Nicot, data not shown). Reciprocal binding between MTM1 and Sec6 as well as PI4K2 $\alpha$ was confirmed by affinity 
chromatography (experiments were done in collaboration with Jocelyn Laporte and AnneSophie Nicot, data not shown). Based on these biochemical data we suggest that a protein module comprising exocyst, PI4K2 $\alpha$ and MTM1, is assembled on endosomes, and functionally involved in endosomal recycling.
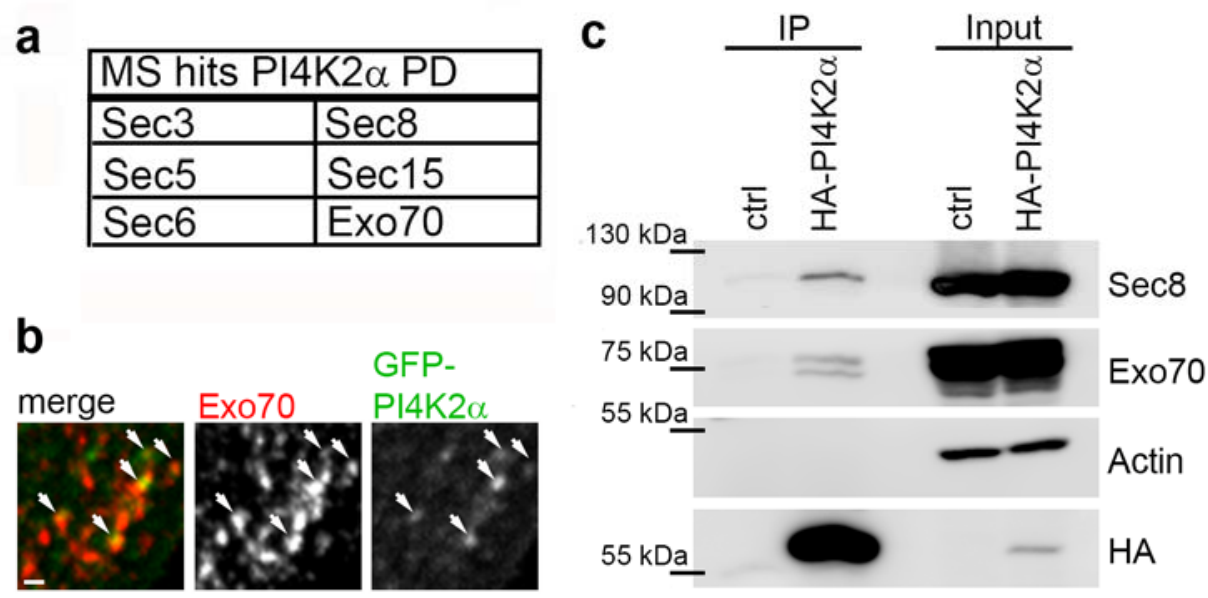

Figure 30: Exocyst as an effector of PI4K2a

(a) Pull down (PD) samples using GST-tagged PI4K2 $\alpha$ as a bait were subjected to mass spectrometry. List of exocyst components found by quantitative mass spectrometry (Mössinger, dissertation). (b) Colocalization of eGFP-PI4K2 $\alpha$ and Exo70 in HeLa cells on endosomes (arrows), assessed by Exo70-specific antibody labeling. Representative images from one of 3 independent experiments are shown. Scale bar: $1 \mu \mathrm{m}$. (c) Coimmunoprecipitation of PI4K2 $\alpha$ and exocyst components from stable Hek293.

To verify this hypothesis, we tested whether exocyst and PI4K $2 \alpha$ were required for endosomal exocytosis. Upon loss of exocyst subunits (i.e. Sec3, Sec6 or Exo70) or of PI4K2 $\alpha$ exocytosis was impaired (Figure 31a-d, g) and perinuclear concentration of TfR-endosomes was lost (Figure 31e, f), akin to the MTM1 loss-of-function phenotype and similar to previous reports on the exocyst complex (Takahashi, Kubo et al. 2012). Furthermore, depletion of PI4K2 $\alpha$ in MTM1 knockdown cells did neither restore perinuclear TfR localization nor exocytosis, but rather aggravated both phenotypes (Figure 31f, g). These data indicate that PI4K2 $\alpha$, MTM1 and exocyst act along the same pathway. Further, they support the model, that assembly of this protein complex on endosomes is necessary for efficient exocytosis. 
a

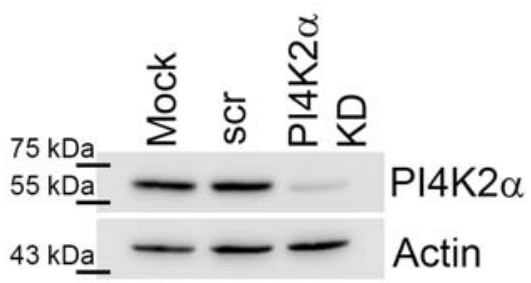

b

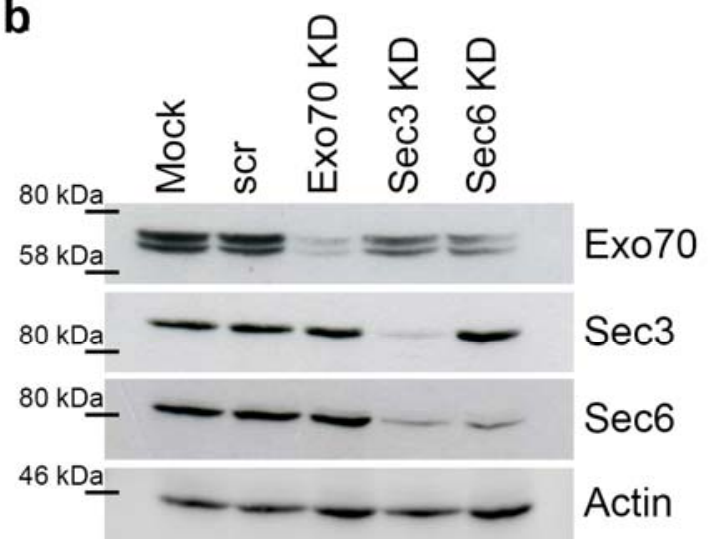

C

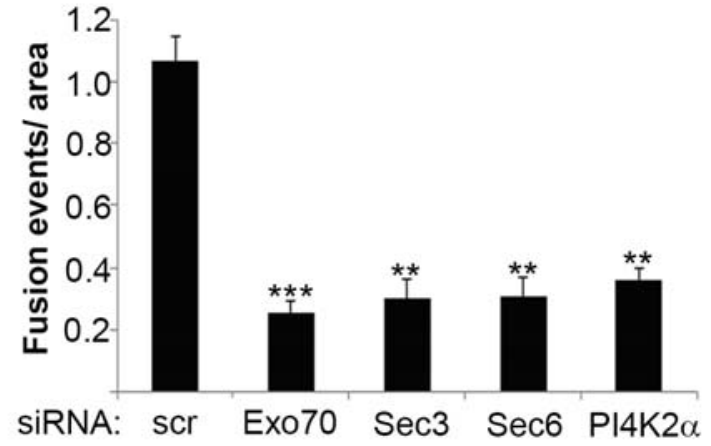

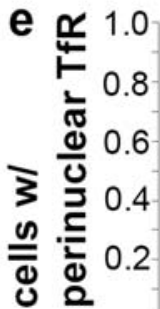

siRNA: scr Exo70 Sec3 Sec6 PI4K2 $\alpha$

SiRNA: scr Exo70 Sec3 Sec6 PI4K2

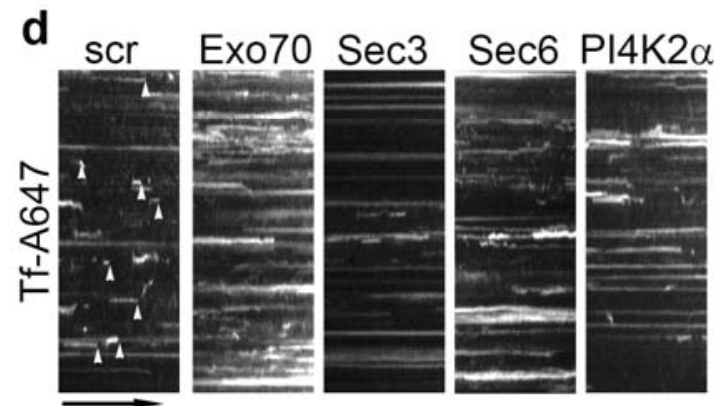

$\overrightarrow{30 \mathrm{sec}}$ $\mathbf{f}$

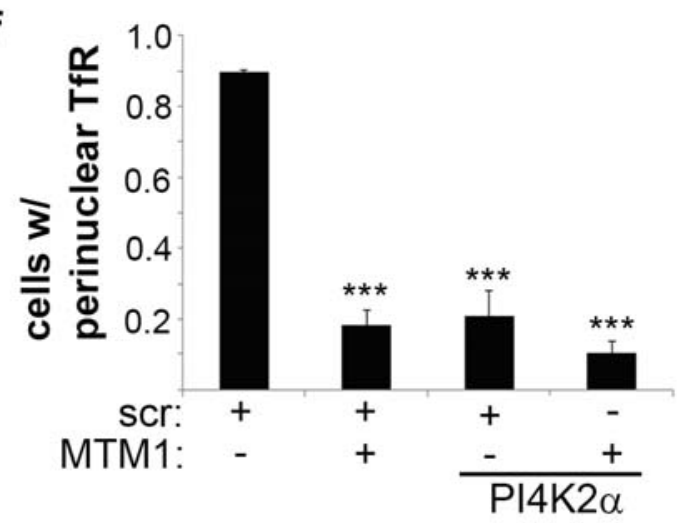

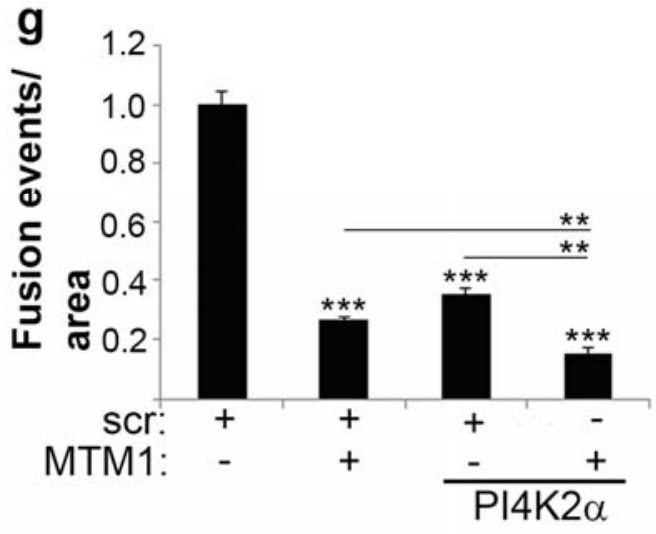

Figure 31: PI4K2 $\alpha$ and exocyst are required for exocytosis

(a,b) Efficient depletion of PI4K2 $\alpha$ (a) and exocyst components Exo70, Sec3 and Sec6 (b) as demonstrated by immunoblotting of HeLa cell lysates. (c,d) Inhibition of Tf exocytosis in cells depleted of PI4K2 $\alpha$ - or exocyst subunits Exo70, Sec3, or Sec6 compared to scrambled (scr) siRNA-treated controls (Tf exocytic events/ area; 
mean $\pm \mathrm{SEM}, n=3$ independent experiments with 5 videos analyzed per condition per experiment, $* * P<0.01$, $* * * P<0.001$, unpaired, two-tailed $t$-test). (d) Kymographs of Tf fluorescence signal over $28 \mu \mathrm{m}$, arrowheads indicate time of plasma membrane fusion. (e) Depletion of PI4K $2 \alpha$ or exocyst from HeLa cells causes TfR misslocalization. Normalized fraction of cells with perinuclear TfR was quantified (mean \pm SEM, $n=4$ independent experiments with 15-30 images analyzed per condition per experiment, $* P<0.05, * * * P<0.001$, unpaired, two-tailed $t$-test). (f) Co-depletion of MTM1 and PI4K2 $\alpha$ does not restore perinuclear TfR localization in MTM1-depleted HeLa cells. Normalized fraction of HeLa cells with perinuclear TfR in cells treated with the indicated siRNAs (mean \pm SEM, $n=3$ independent experiments with 15-30 images analyzed per condition per experiment, $* * * P<0.001$, unpaired, two-tailed $t$-test). (g) Co-depletion of MTM1 and PI4K2 $\alpha$ does not restore defective Tf exocytosis in MTM1-depleted HeLa cells (Tf exocytic events/ area; mean \pm SEM, $n=3$ independent experiments with 5 videos analyzed per condition per experiment, $* * P<0.01, * * * P<0.001$, unpaired, two-tailed $t$-test).

\subsubsection{Rab11 is required for endosomal exocyst localization}

Apart from the presence of PI(4)P, recruitment of exocyst onto endomembranes critically depends on GTP-bound Ypt32p, the yeast homologue of mammalian Rab11

(Mizuno-Yamasaki, Medkova et al. 2010). Further, the exocyst subunit Sec15 had been shown previously to be a direct effector of Rab11a in mammalian cells (Zhang, Ellis et al. 2004).
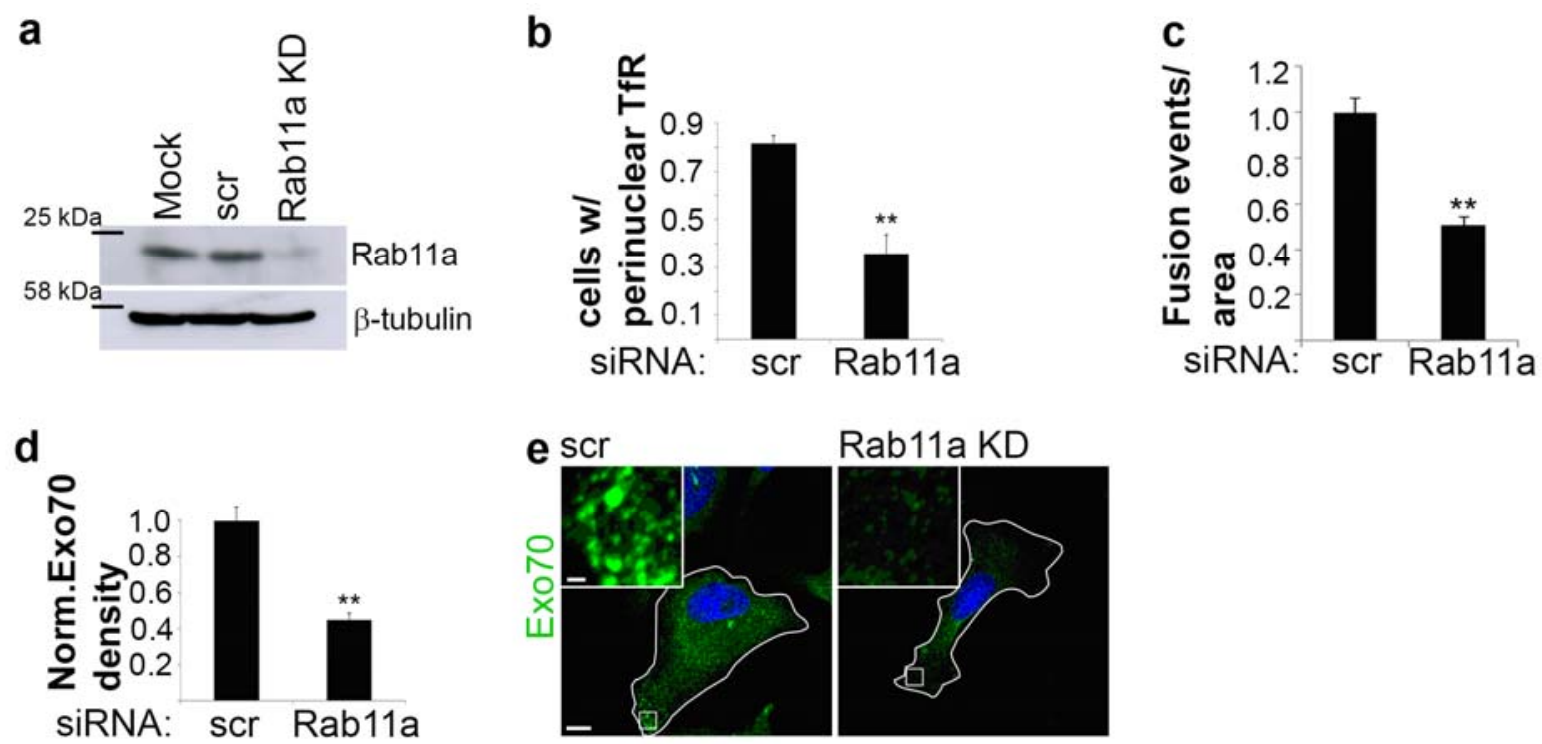

Figure 32: Rab11a-mediated exocyst recruitment to endosomes is required for exocytosis

(a) Efficient siRNA-mediated depletion of Rab11a from HeLa cells as demonstrated by immunoblot analysis. (b) TfR misslocalizes upon loss of Rab11a in HeLa cells. Normalized fraction of HeLa cells with perinuclear TfR in cells treated with the indicated siRNAs (mean \pm SEM, $n=3$ independent experiments with 15-30 images analyzed per condition per experiment, $* * P<0.01$, unpaired, two-tailed $t$-test). (c) Impaired Tf exocytosis monitored by TIRF microscopy in Rab1la-depleted HeLa cells. Quantified are Tf exocytosis events normalized to cell area (mean $\pm \mathrm{SEM}, n=3$ independent experiments with 5 videos analyzed per condition per experiment, $* * P<0.01$, unpaired, two-tailed $t$-test). (d) Reduced endosomal exocyst association in Rab11a-depleted HeLa cells revealed by confocal imaging using Exo70-specific antibody labeling (mean $\pm \mathrm{SEM}, n=3$ independent experiments with 15-30 images analyzed per condition per experiment, $* * P<0.01$, unpaired, two-tailed $t$-test). (e) Representative images from one of 3 independent experiments are shown. Scale bar, $10 \mu$ m; magnified insets, $1 \mu \mathrm{m}$. 
Consistent with further published data (Takahashi, Kubo et al. 2012), perinuclear accumulation of TfR was lost upon loss of Rab11a, TfR endosomes accumulated in the cell periphery and were exocytosis-deficient (Figure 32a-c). As shown by immunocytochemistry staining, exocyst complex, marked by the Exo70 subunit, localized to a very distinct endosome population in the cell periphery in scrambled-siRNA treated controls. Most importantly, this endosomal localization was lost upon Rab11a depletion (Figure 32d, e). Taken together our data thus demonstrate that Rab11a-dependent exocyst recruitment to endosomes is required for endosomal exocytosis.

Directional membrane trafficking frequently conincides with a switch in endosomal PI- as well as Rab-identity (Behnia and Munro 2005). To address the Rab-identity of exocytic vesicles we applied dual-color TIRF microscopy to monitor Tf exocytosis in cells expressing GFP-tagged variants of different Rab proteins. Our data demonstrate that exocytic vesicles frequently acquired Rab11a prior to fusion with the plasma membrane. Loss of Rab11a from exocytic vesicles slightly preceded the release of $\mathrm{Tf}$ upon fusion with the plasma membrane (Figure 33). To confirm this time line, the acquisition order of the Tf- and Rab11-channel was reversed. Indeed, loss of Rab11 slightly preceeded the $\mathrm{Tf}$ (data not shown). This strongly suggests that Rab11a dissociated from vesicles before exocytosis but after assembly of the exocyst complex. This mechanism might ensure that the cell surface remains devoid of Rab11a, a marker of recycling endosomes. Moreover, endosomes positive for Rab5 failed to fuse with the plasma membrane, while endosomes negative for Rab5 frequently underwent exocytosis (Figure 33). As Rab5-positive endosomes are enriched in PI(3)P (Shin, Hayashi et al. 2005), this observation nicely agrees with the observation that $\mathrm{PI}(3) \mathrm{P}$ removal is a prerequisite for surface delivery of endosomal cargo (see Figure 11).

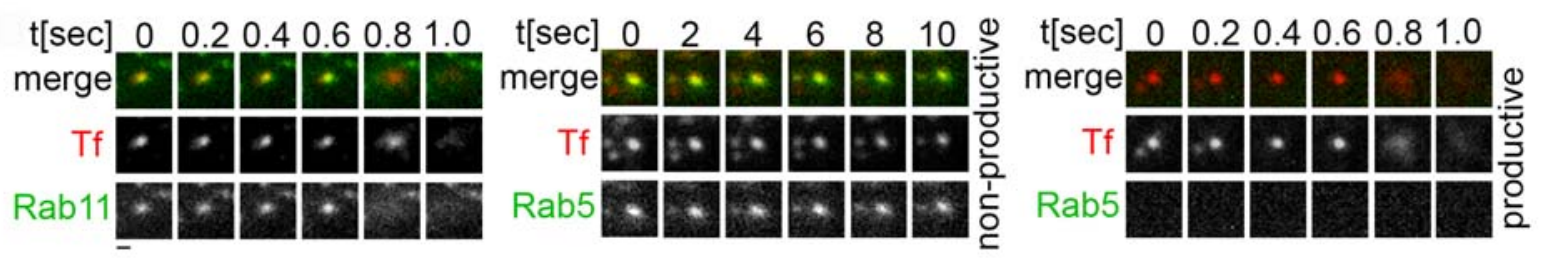

Figure 33: Conversion of Rab5-to-Rab11-containing endosomes during exocytic recycling

Tf exocytosis was monitored by dual-color TIRF microscopy in HeLa cells expressing GFP-Rab5a or GFPRab11a. Snapshots of representative Tf-positive, Rab-containing endosomes are shown. Representative images from one experiment with 10 videos per condition are shown. Scale bar: $400 \mathrm{~nm}$. 


\subsubsection{Exocyst recruitment to endosomes depends on PI4K2 $\alpha$ and PI(4)P}

As exocyst subunits form a complex with both MTM1 and PI4K2 $\alpha$, we next addressed the functional relationship between these proteins. To this aim we analyzed biochemically and by immunocytochemistry whether membrane recruitment of the exocyst complex depends on PI4K2 $\alpha$ and/ or MTM1. Therefore, we performed cell fractionation experiments to separate proteins into cytosolic and membrane-associated fractions. While we could not detect cytosolic actin on purified endomembranes, palmitoylated, membrane-anchored Gadkin was highly enriched in this fraction (Maritzen, Schmidt et al. 2010), illustrating and controlling fractionation efficiency (Figure 34a, c). Upon loss of PI4K2 $\alpha$ the membrane-associated fraction of the exocyst complex (subunits $\operatorname{Sec} 3, \operatorname{Sec} 8$ and Exo70) was significantly reduced. In contrast, MTM1 depletion did not affect its membrane recruitment (Figure 34a, b). Further, the biochemical data could be confirmed by immunocytochemistry stainings. Whereas in control and MTM1-depleted cells the exocyst complex, visualized by the Exo70 subunit, localized to distinct vesicular structures in the cell periphery, this membrane associated Exo70 pool was lost upon loss of PI4K2 $\alpha$ (Figure $34 \mathrm{~d}$, e). Moreover, by using membrane-permeant PIP analogues oversupply of PI(4)P in PI4K2 $\alpha$-depleted cells could restore endosomal localization of exocyst to control levels, whereas the phenotype persisted upon PI(3)Ptreatment (Figure 34f). Consistent with a requirement of PI(4)P, expression of kinase-inactive (D308A) (Balla, Tuymetova et al. 2002) compared to wild-type PI4K2 $\alpha$ led to a displacement of exocyst from endomembranes, presumably by lowering endosomal PI(4)P-levels due to outcompeting the active enzyme (Figure $34 \mathrm{~g}$ ).

These data argue that coincidence detection of PI4K2 $\alpha$ itself, PI4K2 $\alpha$-mediated $\mathrm{PI}(4) \mathrm{P}$-production and Rab11 on recycling endosomes is required for recruitment of the exocyst complex. This mechanism enables fusion of these endosomes with the plasma membrane.

\subsubsection{PI4K2 $\alpha$-mediated MTM1 recruitment to endosomes initiates PI(3)P- dephosphorylation}

According to the data described above, MTM1-mediated removal of PI(3)P and concomitantly of PI(3)P-effector proteins as well as PI4K2 $\alpha$-dependent PI(4)P synthesis are a prerequisite for endosomal recycling en route to the plasma membrane. To decipher how these two processes are coupled, we analyzed their interdependence with regard to the association of MTM1 and PI4K2 $\alpha$ with endomembranes. 


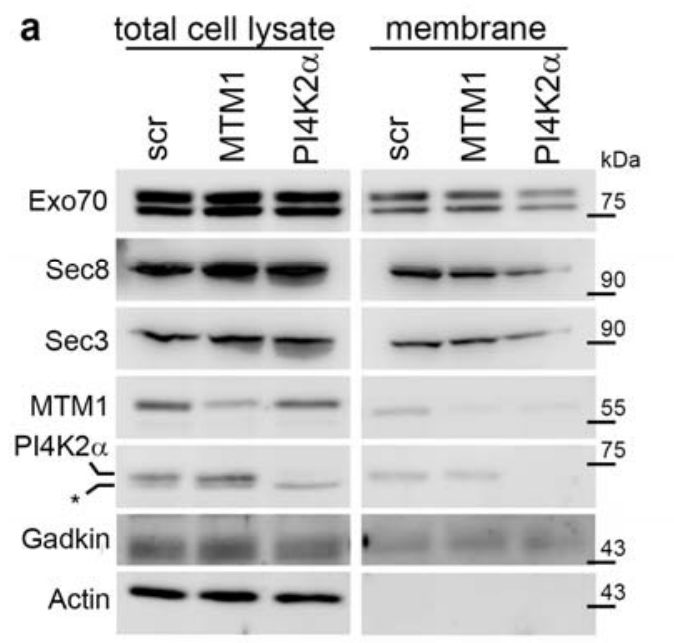

b

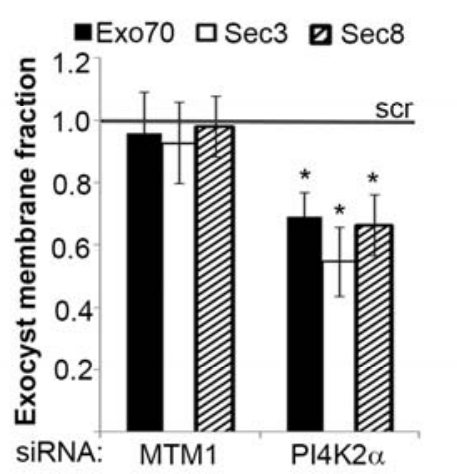

C

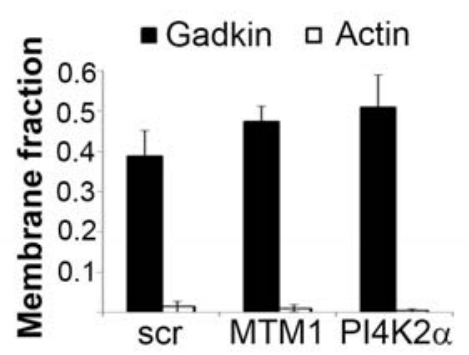

d

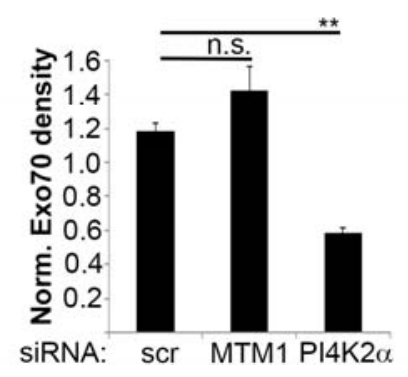

f

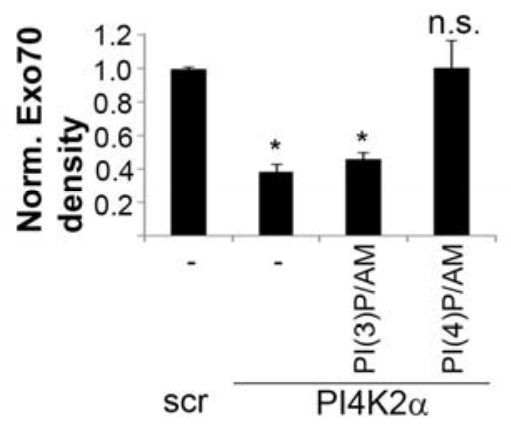

e

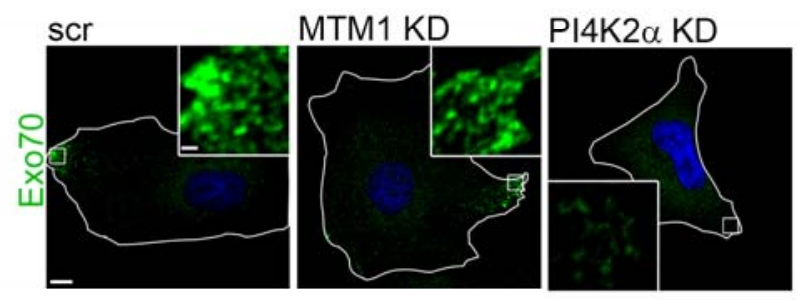

g

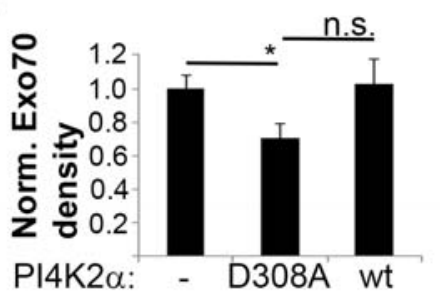

Figure 34: Membrane-recruitment of the exocyst complex depends on PI4K2 $\alpha$ and PI(4)P and is independent of MTM1

(a) Depletion of PI4K2 $\alpha$ but not of MTM1 impairs membrane recruitment of Exo70, Sec8 and Sec3. Representative immunoblot is shown. (*), non-specific band recognized by PI4K2 $\alpha$ antibodies. (b) Relative membrane associated fraction of exocyst subunits Exo70, Sec8, or Sec3 in (scr) control, MTM1-depleted, or PI4K2 $\alpha$-depleted cells (mean $\pm \mathrm{SEM}, n=5$ independent experiments, ${ }^{*} P<0.05$, one-sample $t$-test). (c) Efficient membrane-cytosol fractionation verified by quantifying the membrane/ total protein ratio of the palmitoylated membrane protein Gadkin and cytosolic actin (mean $\pm \mathrm{SEM}, n=5$ independent experiments). (d) Reduced endosomal exocyst association in PI4K2 $\alpha$-depleted but not in MTM1-depleted cells revealed by confocal imaging using Exo70-specific antibody labeling (mean $\pm \mathrm{SEM}, n=3$ independent experiments with 15-30 images analyzed per condition per experiment, ${ }^{* *} P<0.01$, unpaired, two-tailed $t$-test). (e) Depletion of PI4K2 $\alpha$ but not of MTM1 in HeLa cells leads to decreased Exo70 level. Representative images from one of 3 independent experiments are shown. Scale bar, $10 \mu \mathrm{m}$; magnified insets, $1 \mu \mathrm{m}$. (f) Reduced endosomal exocyst association in PI4K2 $\alpha$ depleted cells is rescued by membrane-permeant $\mathrm{PI}(4) \mathrm{P}$ but not $\mathrm{PI}(3) \mathrm{P}$ (mean $\pm \mathrm{SEM}, n=$ 4 independent experiments with 15-30 images analyzed per condition per experiment, $* P<0.05$, unpaired, twotailed $t$-test). (g) Expression of mutant inactive (D308A) but not wild-type (wt) PI4K2 $\alpha$ in HeLa cells reduces endosomal exocyst association revealed by confocal imaging using Exo70-specific antibody labeling (mean \pm SEM, $n=3$ independent experiments with 15-30 images analyzed per condition per experiment, $* P<0.05$, unpaired, two-tailed $t$-test). 
Loss of PI4K2 $\alpha$ impaired membrane recruitment of MTM1 in cell fractionation experiments (Figure 34a, 35a). Moreover, the membrane-associated fraction of MTM1 was very low under control conditions. When PI4K2 $\alpha$ was overexpressed a significantly higher pool of MTM1 was recruited to Rab5-containing endosomes. However, this recruitment was independent of the catalytic activity of PI4K2 $\alpha$, indicating that MTM1-recruitment mainly depends on MTM1-PI4K2 $\alpha$ complex formation rather than on MTM1 binding to PI(4)P (Figure 35b). In line with this, in vitro liposome binding studies on MTM1 showed only minor affinity for PI(4)P compared to PI(3)P (data not shown, experiments performed by Dr. Michael Krauß). On the contrary, PI4K2 $\alpha$ remained associated with membranes and remained localized to TfR-containing endosomes in MTM1-depleted cells (Figure 35c). These observations suggest a tight regulation of the endosomal recruitment of MTM1 by PI4K2 $\alpha$ and implicate that endosomal PI4K2 $\alpha$ triggers MTM1-mediated PI(3)P-hydrolysis.

a

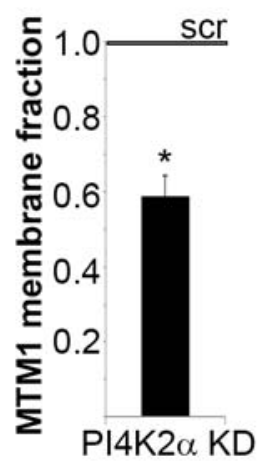

b

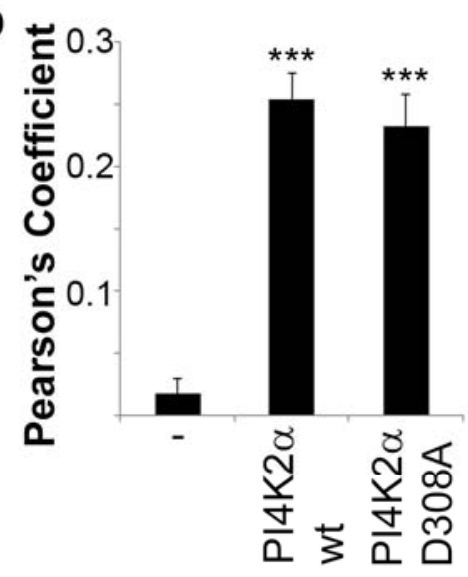

MTM1 KD

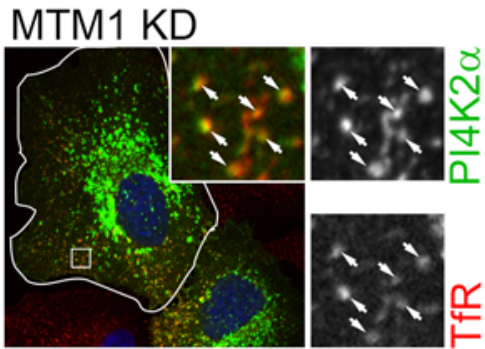

Figure 35: PI4K2 $\alpha$-dependent endosomal recruitment of MTM1

(a) Relative membrane associated fraction of MTM1 in (scr) control or PI4K2 $\alpha$-depleted cells addressed biochemically in membrane fractionation experiments (mean \pm SEM, $n=5$ independent experiments with 15-30 images analyzed per condition per experiment, ${ }^{*} P<0.05$, unpaired, two-tailed $t$-test). Representative immunoblot is shown in Figure 28a. (b) PI4K2 $\alpha$-dependent recruitment of MTM1 is independent of its kinase activity. Expression of wild-type (wt) or inactive, mutant (D308A) eGFP-PI4K2 $\alpha$ recruits mCherry-MTM1 to Rab5 Q79L endosomes. Quantified are Pearson's Coefficients between mCherry-MTM1 and HA-Rab5 Q79L (mean \pm SEM, $n=4$ independent experiments with 15-30 images analyzed per condition per experiment, $* * * P<$ 0.001, unpaired, two-tailed $t$-test). (c) eGFP-PI4K2 $\alpha$ expressed in MTM1-depleted HeLa cells localizes to TfRpositive endosomes, assessed by TfR-specific antibody labeling and indicated by arrows. Representative images from one of 4 independent experiments are shown. Scale bar, $10 \mu \mathrm{m}$; magnified insets, $1 \mu \mathrm{m}$. 

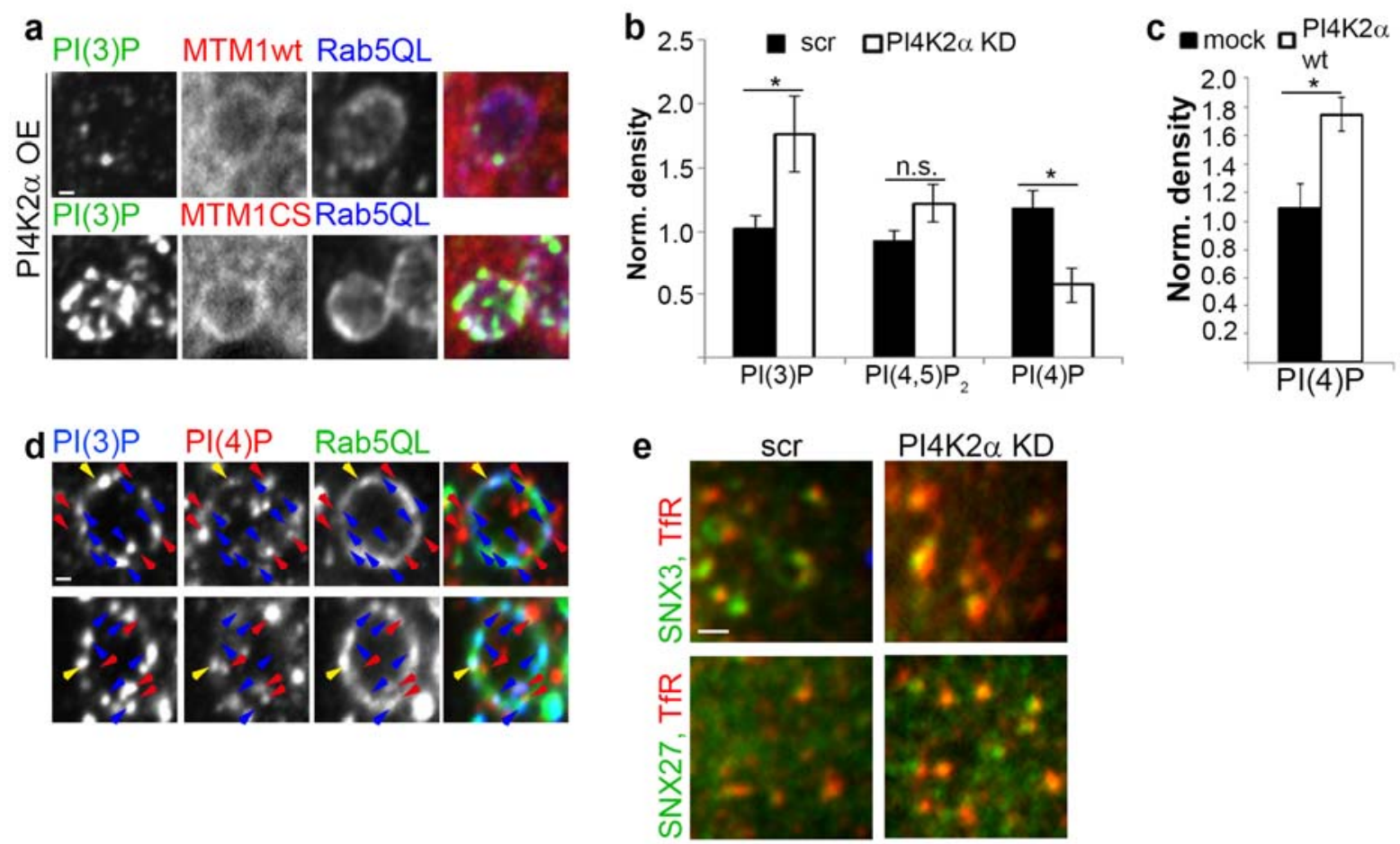

Figure 36: PI4K2 $\alpha$-mediated recruitment of MTM1 initiates PI(3)P-to-PI(4)P conversion on endosomes

(a) HeLa cells co-expressing wild-type (wt) or inactive, mutant (C375S, CS) MTM1, PI4K2 $\alpha$ and Rab5 Q79L (QL) were labeled for PI(3)P using eGFP-2xFYVE. Inactive MTM1 localizes to PI(3)P-positive early endosomes, while this PI(3)P pool is lost upon recruitment of active MTM1. Representative images from one experiment with 10-20 images per condition are shown. Scale bar: $1 \mu \mathrm{m}$. (b) Selective decrease of PI(4)P and increase of PI(3)P in PI4K2 $\alpha$-depleted HeLa cells. Shown are the relative levels of PI(3)P, PI(4)P and PI(4,5) $\mathrm{P}_{2}$ normalized to cell area (mean \pm SEM, $n=3$, for PI(3)P: $n=7$ independent experiments with 15-30 images per condition per experiment, $* P<0.05$, n.s.: non-significant, unpaired, two-tailed $t$-test). (c) Increased PI(4)P levels in HeLa cells expressing wild-type (wt) eGFP-PI4K2 $\alpha$ compared to mock transfected cells. Increase of PI(4)P is consistent with PI 4-kinase activity of PI4K2 $\alpha$. Shown are the relative levels of PI(4)P normalized to cell area (mean \pm SEM, $n=3$ independent experiments with 15-30 images per condition per experiment, $* P<0.05$ unpaired, two-tailed $t$-test). (d) PI(3)P and PI(4)P localize to distinct subdomains on Rab5 Q79L endosomes labeled using eGFP-2xFYVE and a PI(4)P-specific antibody respectively. PI localizations are indicated by arrow heads (blue: PI(3)P; red: PI(4)P; yellow: colocalization of PI(3)P and PI(4)P). Representative images from one experiment with 10-20 images per condition are shown. Scale bar: $1 \mu \mathrm{m}$. (e) Recruitment of eGFP-SNX3 and eGFP-SNX27 to dispersed endosomes in PI4K2 $\alpha$-depleted HeLa cells. Representative images from one experiment with 10-20 images per condition are shown. Scale bar, $2 \mu \mathrm{m}$.

Finally, we wanted to determine how PI4K2 $\alpha$-facilitated membrane recruitment of MTM1 regulates endosomal PI(3)P and PI(4)P levels. PI4K2 $\alpha$ - and Rab5-containing endosomes were highly enriched in PI(3)P upon recruitment of phosphatase-inactive, mutant (C375S) MTM1 (Figure 36a). This matches the established PI-identity of the early endosomal compartment. Besides, recruitment of active, wild-type MTM1 caused depletion of PI(3)P from these endosomes (Figure 36a). Importantly, semi-quantitative analysis of total PI levels in PI4K2 $\alpha$-depleted cells revealed not only a reduction in PI(4)P, consistent with its PI 4kinase activity (Balla, Tuymetova et al. 2002), but also a prominent increase in PI(3)P (Figure 
36b). Further, expression of active PI4K2 $\alpha$ elevated PI(4)P (Figure 36c). Thus, our data strengthens the correlation between PI4K2 $\alpha$ and endosomal PI(4)P levels - as detected by PI(4)P-specific antibody labeling - and indicates that this PI(4)P pool is synthesized by PI4K2 $\alpha$. Furthermore, dispersed TfR-endosomes in PI4K2 $\alpha$-depleted HeLa cells contained PI(3)P-binding effector proteins of the SNXs family, i.e. SNX3 and SNX27 (Figure 36e). These data illustrate that impaired endosomal recruitment of MTM1 upon loss of PI4K2 $\alpha$ (see Figure 34a, 35a) mimics the MTM1 loss-of-function phenotype (see Figure 18). This includes the elevation of $\mathrm{PI}(3) \mathrm{P}$ levels and the endosomal accumulation of $\mathrm{PI}(3) \mathrm{P}$-effector proteins.

Although Rab5-positive endosomes were predominantly enriched in PI(3)P, we were also able to detect PI(4)P. Both lipids were contained in distinct endosomal subdomains with only minor overlap (Figure 36d). Moreover, PI4K2 $\alpha$ and PI(4)P were found within the same early endosomal domain (Figure 37a), further indicating that PI4K2 $\alpha$ generates this endosomal PI(4)P pool. In addition to PI(3)P-positive Rab5 endosomes containing PI4K2 $\alpha$ and MTM1, an additional endosomal population exists that is devoid of Rab5 but contains PI4K2 $\alpha$ and MTM1 (Figure 37b). Presumably, these are Rab11-positive recycling endosomes emanating from the early endosome. Thus, we hypothesize that PI4K2 $\alpha$-mediated PI(4)Psynthesis on distinct endosomal subdomains favors progression from these domains towards endosomal recycling.

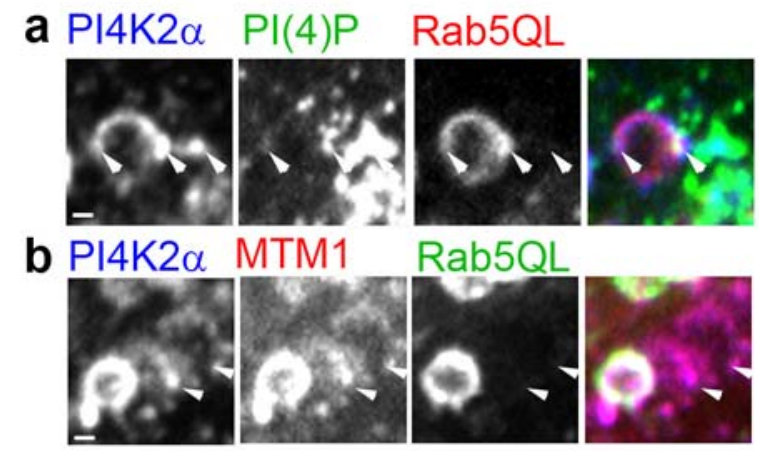

Figure 37: Early endosomal PI(4)P is synthesized by PI4K2a

(a) HeLa cells co-expressing GFP-PI4K2 $\alpha$ and Rab5 Q79L (QL) were labeled for PI(4)P using PI(4)P-specific antibodies. Colocalization between PI4K2 $\alpha$ and PI4P on Rab5QL endosomes is indicated by arrowheads. Representative images from one experiment with 10-20 images per condition are shown. Scale bar: $1 \mu \mathrm{m}$. (b) MTM1, PI4K2 $\alpha$ and Rab5 Q79L (QL) were coexpressed in HeLa cells. Rab5-negative endosomes containing PI4K2 $\alpha$ and MTM1 are indicated by arrowheads. Representative images from one experiment with 10-20 images per condition are shown. Scale bar: $1 \mu \mathrm{m}$. 


\section{Discussion}

Endosomal membrane traffic crucially depends on conversion of membrane identity defined by small Rab GTPases and PIs. For clathrin-mediated endocytosis and early to late endosomal maturation, interconversion of PIs is a well established concept. Yet, how PI composition on early endosomes (enriched in $\mathrm{PI}(3) \mathrm{P})$ is converted en route to the cell surface (predominantly 4'-phosphorylated PI species, i.e. PI(4)P and $\left.\mathrm{PI}(4,5) \mathrm{P}_{2}\right)$ remained enigmatic. In the present work we identified a protein module, comprising MTM1, PI4K2 $\alpha$ and the exocyst complex, that is required for PI conversion from PI(3)P to PI(4)P during endosomal recycling. Simultaneously, Rab identity switches from a Rab5-positive early to a Rab11positive recycling endosome, prior to exocyst-dependent fusion with the PM. Furthermore, MTM1, PI4K2 $\alpha$ and exocyst are regulated by reciprocal interactions and coincidence detection of small Rab GTPases and PIs (Figure 38, highlighted in (Balla 2016)).

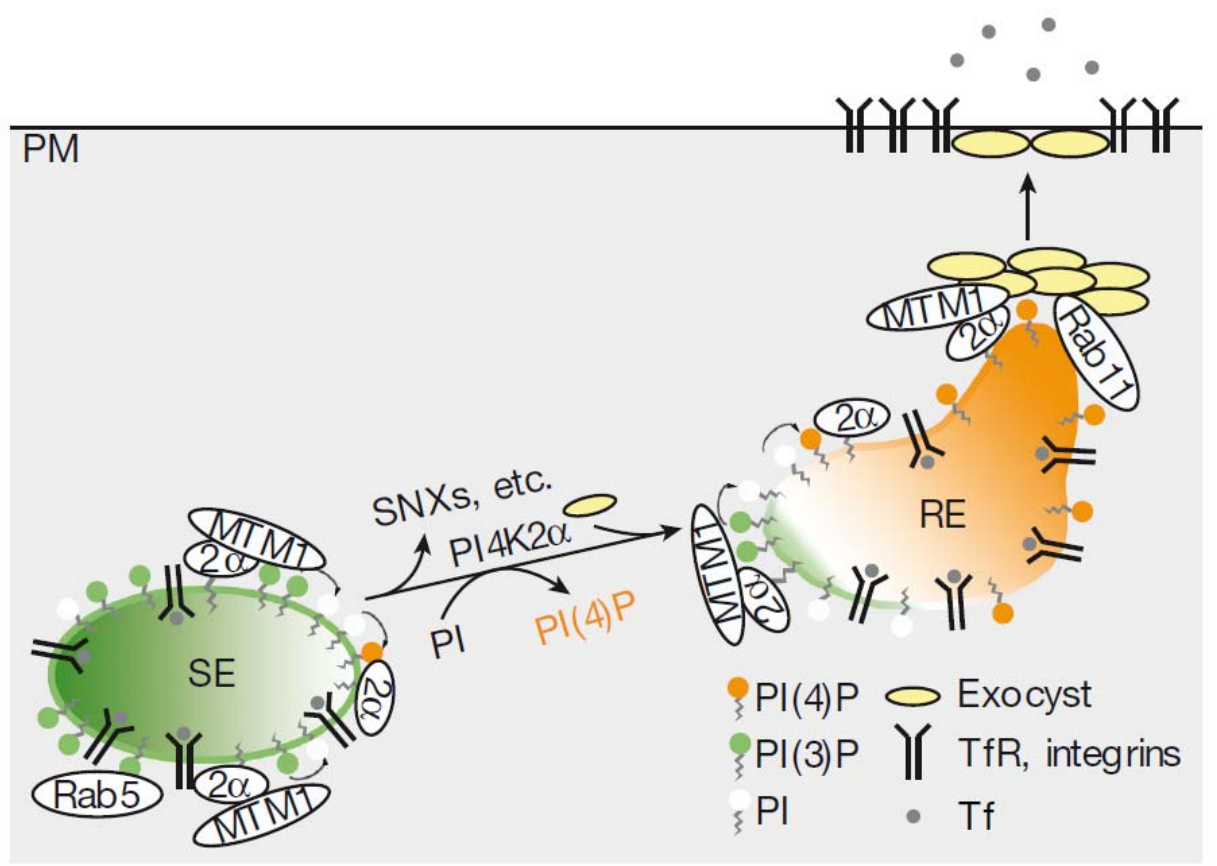

\section{Figure 38: PI(3)P-to-PI(4)P conversion in endosomal exocytosis}

$2 \alpha$ - PI4K2 $\alpha$; PM - plasma membrane; RE - recycling endosome; SE - sorting endosome; SNXs - sorting nexins; Tf - Transferrin; TfR - Tf receptor.

As the primary underlying cause of XLCNM is still debated this study provides strong evidence that defective exit from endosomes caused by MTM1 loss-of-function could be of 
crucial importance. As these defects are reversed by VPS34-IN1 treatment, these results might have implications for combating XLCNM. Further, this study substantially contributes to understanding the regulation of endosomal PI(3)P and PI(4)P levels, the cellular roles of MTM1 and PI4K2 $\alpha$ as well as other members of the myotubularin family. In general, the concept of PI conversion might be applied to other trafficking routes regulated by multiple PI species.

\subsection{Regulation of endosomal PI(3)P and PI(4)P is tightly coupled}

\subsubsection{Endosomal PI(3)P levels are balanced by 3'-metabolizing enzymes}

Endosomal membrane identity is partly defined by their predominant PI species (Behnia and Munro 2005). As membrane traffic constantly occurs between endosomal membranes, PI content of the donor compartment has to be converted to match the acceptor compartment identity. The early endosome is highly enriched in PI(3)P. Maintenance of these high levels was attributed to the class III PI 3-kinase Vps34, an early endosomal Rab5effector that synthesizes PI(3)P from PI de novo (Shin, Hayashi et al. 2005). While PI(3)P synthesis by Vps34 is required for generation of the endosomal compartment (Christoforidis, Miaczynska et al. 1999), its depletion is required for exit from endosomes, most notably for endosomal recycling. Here, a local burst of MTM1-mediated PI(3)P depletion on endosomes, initiated upon PI4K2 $\alpha$-dependent recruitment, is essential for cargo exit from early endosomes. In addition to Vps34 and MTM1, the class II PI3Ks and myotubularin-related PI 3-phosphatases have been localized to endosomal membranes. Interestingly, how myotubularins, class II and III PI3Ks partition in turn-over of the endosomal PI(3)P pool remains to be clarified. To address the cooperation of MTM1 and Vps34 in early endosomal PI(3)P regulation, we used the Vps34-specific inhibitor VPS34-IN1 or Vps34 depletion in MTM1-KD cells. While both treatments rescued defective exocytosis upon MTM1-loss-offunction, the latter only partially restored normal, perinuclear TfR localization (see Figure 23). Thus, the type II PI3Ks might provide an alternative source for de novo PI(3)P synthesis on endosomes. In line with this, Johnson et al. showed that Vps34 depletion caused only minor alterations in endosomal association of EEA1 and the PI(3)P-specific FYVE-domain (Johnson, Overmeyer et al. 2006). Only when applying the broad spectrum PI3K inhibitor wortmannin, which affects all three classes of PI3Ks, EEA1 was lost from endosomes. Notably, co-depletion of the type II PI3Ks in Vps34-KO mouse embryonic fibroblasts (MEFs) 
further reduced total PI(3)P level (Devereaux, Dall'Armi et al. 2013). Accordingly, depletion of type II and III PI3Ks in D. melanogaster had additive effects on cellular PI(3)P levels in mtm-null mutants (Velichkova, Juan et al. 2010). Alternatively to PI(3)P-synthesis on endosomes, 4'-dephosphorylation of $\mathrm{PI}(3,4) \mathrm{P}_{2}$, generated by PI3KC2 $\alpha$ during CME (Posor, Eichhorn-Gruenig et al. 2013) and potentially PI3KC2 $\beta$ at the PM (Wheeler and Domin 2006; Nakatsu, Perera et al. 2010), feeds into the endosomal PI(3)P pool. Taken together, these data indicate that multiple synthesis routes contribute to the total endosomal PI(3)P pool. As the endosomal compartment comprises early, sorting, tubular recycling and late endosomes, it is conceivable to assume that the class II and III PI3Ks act on different endosomal subpopulations or even distinct endosomal sorting tubules.

In contrast to its crucial function in endosomal PI(3)P turnover, MTM1 depletion led to a comparably small increase in total PI(3)P levels (see Figure 21), in line with previous reports (Cao, Backer et al. 2008). In addition to MTM1 depletion, loss of the endosomal PI(3)P-specific 3-phosphatases MTMR2 or MTMR4 has been shown to elevate cellular PI(3)P levels (Cao, Backer et al. 2008; Naughtin, Sheffield et al. 2010). Thus, other myotubularins might compensate for loss of MTM1 as was proposed previously (Laporte, Liaubet et al. 2002). However, apart from MTMR1, which resulted from MTM1-gene duplication, neither loss of MTMR2, MTMR4 nor MTMR7 could phenocopy MTM1 depletion with respect to endosomal exocytosis (see Figure 15). Thus, whereas MTM1 acts predominantly at the level of early and recycling endosomes in exocytic transport, other myotubularins might be crucial for spatial and temporal regulation of PI(3)P-turnover along further endolysosomal transport routes. MTMR2 preferentially localizes to PI(3)P- and $\mathrm{PI}(3,5) \mathrm{P}_{2}$-positive late endosomes and prefers $\mathrm{PI}(3,5) \mathrm{P}_{2}$ as a substrate (Berger, Berger et al. 2006; Cao, Backer et al. 2008). Accordingly, MTMR2 could mediate degradative sorting or recycling from $\mathrm{PI}(3,5) \mathrm{P}_{2}$-positive late endosomes (as discussed in 4.3.1). MTMR4, on the other hand, was shown to predominantly localize to early endosomes, but its depletion did not alter the overall recycling kinetics of TfR (Naughtin, Sheffield et al. 2010). However, prior to monitoring exocytosis, we load the endosomal system with internalized ligand until saturation is reached. Thus, our exocytosis assay might favour the slow recycling pathway via Rab11endosomes. According to this assumption, MTMR4 might selectively regulate the fast recycling route from early endosomes directly back to the PM while its loss is compensated by favouring the slow recycling pathway. Alternatively, it could be implicated in early endosomal sorting or recycling of other endosomal cargo irrespective of TfR. Interestingly, MTMR7 was localized to Golgi-like structures (Mochizuki and Majerus 2003). Thus, 
MTMRs might be implicated in retrograde endosome to TGN transport or maintenance of low PI(3)P levels at the TGN.

Taken together, these results suggest a synergistic role of myotubularins, type II and III PI 3-kinases in endosomal PI(3)P homeostasis. Importantly, it needs to be clarified how distinct intracellular PI(3)P pools on endosomal subpopulations are distinguished and regulated by 3'metabolising enzymes.

\subsubsection{Membrane recruitment of MTM1 is regulated by lipid and protein content}

Although we localized MTM1 to early and recycling endosomes (see Figure 19), its majority remains cytosolic. The latter conclusion is corroborated by previous studies on MTM1 localization (Cao, Laporte et al. 2007) and further applies to other myotubularins. This indicates a tight regulation of their membrane association and thereby enzymatic activity.

Membrane localization can be regulated by either its lipid content or membrane associated proteins. Binding of MTM1 or MTMR2 to the regulatory subunit of Vps34, Vps15, is crucial for their localization to early and late endosomes, respectively, and regulates PI 3-kinase as well as PI 3-phosphatase activity (Cao, Backer et al. 2008). Furthermore, Sbf2, the Drosophila homologue of the pseudo-phosphatase MTMR13, is required for membrane localization of the class II PI3K Pi3K68D and $\mathrm{mtm}$, homologues to mammalian MTM1, MTMR1 and MTMR2 (Jean, Cox et al. 2012). Thus, recruitment of myotubularins by PI 3kinases might be required for locally and temporally restricted PI(3)P turnover while maintaining the overall, endosomal PI(3)P content. Future experiments will be needed to systematically analyse the functional correlation between PI 3-kinases and 3-phosphatases as this co-regulation presumably applies to all members of the myotubularin family. It remains to be clarified how PI(3)P turnover is achieved by coexistence of 3'-metabolizing enzymes on endosomal membranes without abrogating its turnover. Notably, we could enhance or abolish the association of MTM1 with early endosomes by PI4K2 $\alpha$ expression and depletion, respectively (see Figures 35, 36). Additionally, MTMR2 and its regulatory MTMRs, MTMR5 and MTMR13, were identified as potential PI4K2 $\alpha$ interactors (dissertation Dr. Julia Mössinger, FU Berlin, 2012). This data suggests that similarly to the Vps34-MTM1-PI4K2 $\alpha$ complex on early endosomes, a Vps34-MTMR2-PI4K2 $\alpha$-complex might regulate transport and recycling from late endosomes. Thus, a systematic and comprehensive analysis of the myotubularin-PI kinase interactome should include all endosomal PI kinases such as type II PI4Ks, late endosomal PIKfyve or lysosomal PI4K3 $\beta$ (Sridhar, Patel et al. 2013) besides PI3Ks. 
Secondly, membrane localization can be regulated by the compartment-specific lipid composition. Despite the fact that analysis of the PI-binding proteome by quantitative mass spectrometry identified MTM1 as a PI(4)P-specific binder (Jungmichel, Sylvestersen et al. 2014), liposome flotation assays at least in our hands failed to reveal selective binding to PI(4)P. Instead, MTM1 preferentially bound to its substrate PI(3)P (data not shown, experiments done in collaboration with Dr. Michael Krauß). Cooperative effects with other lipids and/ or proteins and lipid head group presentation might modulate PI binding affinities of MTM1 within the given subcellular context. In this regard, cholesterol content has been shown to be essential for membrane association of PI4K2 $\alpha$ (Lu, Sun et al. 2012). It could further impact the presentation of lipid head groups (Czogalla, Grzybek et al. 2014), might define PI4K2 $\alpha$-enriched endosomal subdomains and thus, modulate MTM1-lipid binding. Thus, within the subcellular context an endosomal pool of PI(4)P synthesized by PI4K2 $\alpha$ could be an additional requirement for the recruitment of MTM1 to endosomes.

Although segregation in distinct endosomal subdomains and/ or timed recruitment paired with regulation of their enzyme activity might be essential for myotubularin functions, the criteria defining these subdomains as well as the mechanistic details remain elusive. Additional endosomal cues, such as inactive MTMRs, endosomal adaptor proteins or further lipid species, could be important. Thereby, they might add to the spatiotemporal regulation of MTM1 and MTMRs required for locally and temporally restricted PI(3)P-depletion.

\subsubsection{PI(3)P and PI(3,5) $P_{2}$ are lipid substrates of MTM1}

In addition to PI(3)P-enriched early endosomes, MTM1 localizes to PI(3)P and $\mathrm{PI}(3,5) \mathrm{P}_{2}$-positive late endosomes (see Figure 19). Thus, it is important to address the substrate specificity of MTM1. Most conclusively, Cao et al showed that MTM1 targets both PI(3)P and PI(3,5) $\mathrm{P}_{2}$ with predominant activity towards PI(3)P (Cao, Backer et al. 2008), which is supported by earlier studies (Blondeau, Laporte et al. 2000; Taylor, Maehama et al. 2000; Kim, Taylor et al. 2002), as well as by our data (see Figures 20d, 21a). Although our data strongly suggests a predominant early endosomal and PI(3)P-dependent role of MTM1, we could not exclude late endosomal and $\mathrm{PI}(3,5) \mathrm{P}_{2}$-dependent functions. In line with an activity towards $\mathrm{PI}(3,5) \mathrm{P}_{2}$, the GRAM-domain of MTM1 shows some specificity for this particular PI (Tsujita, Itoh et al. 2004). Further, addition of PI(5)P, its potential enzymatic product, can stimulate its 3-phosphatase activity towards $\mathrm{PI}(3) \mathrm{P}$ and $\mathrm{PI}(3,5) \mathrm{P}_{2}$ (Schaletzky, Dove et al. 2003). Interestingly, we identified the PI(3,5) $\mathrm{P}_{2}$ 5-phosphatase Fig4, PIKfyve, the scaffold and activator of PIKfyve, ArPIKfyve/ Vac14, (Appendix C, Table 11, experiments 
done in collaboration with Eberhard Krause, FMP Berlin) and MTMR2 (dissertation Dr. Julia Mössinger, FU Berlin 2012) as potential PI4K2 $\alpha$ interactors. Thus, similarly to the MTM1PI4K2 $\alpha$ complex, which we propose to act in PI(3)P turnover on early endosomes, a MTM1/ MTMR2-PI4K2 $\alpha$ complex in cooperation with the PIKfyve complex might function in PI(3)P and $\mathrm{PI}(3,5) \mathrm{P}_{2}$ turnover on late endosomes. Furthermore, some studies on MTM1 substrate specificity showed a slight activity towards $\mathrm{PI}(3,4) \mathrm{P}_{2}$ (Taylor, Maehama et al. 2000; Kim, Taylor et al. 2002), a PI species of so far ill defined endosomal function and localization. Nevertheless, the type II PI3Ks might generate such an endosomal PI(3,4) $\mathrm{P}_{2}$ pool that could define endosomal subpopulation and could be required for endosomal sorting and/ or signaling. Future research will be needed to clarify the substrate specificity of MTM1 and if or how sub-compartmental localization might change its substrate preferences.

Enzymatic activity of active myotubularins is thought to be regulated by homodimerization (Schaletzky, Dove et al. 2003) and hetero-dimerization with inactive members of the myotubularin family. So far, only MTMR12 has been identified as a binding partner of MTM1, and has been shown to sequester MTM1 in the cytosol upon over-expression (Nandurkar, Layton et al. 2003). Accordingly, we identified MTMR12 as potential interaction partner of MTM1 by quantitative mass spectrometry, but also MTMR10, an inactive myotubularin of so far unknown function, and MTMR1 (Appendix C, Table 11, experiments done in collaboration with Eberhard Krause). MTM1-MTMR10 versus -MTMR12 binding might i.e. regulate targeting of MTM1 to early versus late endosomes, might modulate MTM1-substrate preference or define endosomal subdomains for MTM1-dependent transport. Furthermore, MTMR10/ MTMR12 might favour association of MTM1 with distinct endosomal PI kinases and thereby define MTM1 function. Complex formation between MTM1 and MTMR1 versus MTM1-homodimerisation might further fine tune MTM1 phosphatase activity. This could be reflected in developmental and tissue-specific regulation of MTM1 and MTMR1 expression as well as sub-compartmental targeting of MTM1 and/ or MTMR1. Such complex formation might partly explain our findings, that, although less severely, depletion of MTMR1 phenocopied MTM1 loss-of-function (see Figure 15).

\subsubsection{PI4K2 $\alpha$-dependent PI(4)P synthesis defines endosomal subdomains for cargo sorting}

When we analyzed the endosomal lipid content, we found PI(3)P and PI(4)P coexisting on early endosomes, but enriched in distinct endosomal subdomains with minimal overlap (see Figure 36). This early endosomal PI(4)P pool is synthesized by PI4K2 $\alpha$ (Henmi, 
Morikawa et al. 2016), a PI 4-kinase that localizes to endosomes in its active, palmitoylated form. In line with this, PI(4)P and PI4K2 $\alpha$ are found within the same endosomal subdomains. Thus, balanced activity of PI4K2 $\alpha$ and the early endosomal PI(4)P 4-phosphatase Sac2 (Hsu, $\mathrm{Hu}$ et al. 2015) on early endosomes might assure low steady state PI(4)P levels while local enrichment of PI(4)P is crucial for cargo sorting as well as conversion of endosomal identity. As discussed in section 4.1.2, PI4K2 $\alpha$ together with early endosomal PI(3)P localizes MTM1 to Rab5-positive endosomes. In turn, 3'-dephosphorylation of PI(3)P by MTM1 locally provides substrate for $\mathrm{PI} 4 \mathrm{~K} 2 \alpha$-mediated $\mathrm{PI}(4) \mathrm{P}$ synthesis, thereby coupling $\mathrm{PI}(3) \mathrm{P}$ and $\mathrm{PI}(4) \mathrm{P}$ turnover. In line with this assumption, we found PI(3)P- and Rab5-positive endosomes containing both PI4K2 $\alpha$ and MTM1 next to PI4K2 $\alpha$-, MTM1-containing endosomes devoid of these early endosomal markers. Although we were unable to directly visualize it, these endosomes presumably contained PI(4)P and recycling endosomal Rab11. Interestingly, PI4K2 $\alpha$ and MTM1 can be found on late endosomes. Thus, these Rab5-negative compartments could be of late endosomal or lysosomal identity and implicate this module in i.e. late endosomal PI turnover, recycling from late endosomes (see section 4.3.1) or lysosomal degradation. However, this seems unlikely as MTMR2 amongst the myotubularin family members is most prominently associated with the late endosomal and lysosomal system. In a recent study, Balla et al. proposed a model as to how PI4K2 $\alpha$ might distinguish between degradative sorting and endosomal recycling (Jovic, Kean et al. 2014). Association of PI4K2 $\alpha$ with the early and recycling endosomal v-SNARE VAMP3 is required for recycling, while on the other hand its association with VAMP7 regulates lysosomal cargo sorting towards late endosomes (Craige, Salazar et al. 2008). Thus, association of PI4K2 $\alpha$ with distinct SNARE complexes determines the formation of PI4K2 $\alpha$-dependent endosomal subdomains. Further, it favours the idea that PI4K2 $\alpha$ interacts and recruits distinct myotubularins to early (MTM1) versus late endosomes (i.e. MTMR2) and thereby mediate endosomal sorting.

The closest homologue of PI4K $2 \alpha$ in mammals is PI4K2 $\beta$, which localizes to endosomes and the Golgi complex and provides an alternative source for endosomal PI(4)P synthesis. So far, AP-1-dependent transport between the TGN and endosomes has been shown to be regulated by PI4K2 $\beta$. On the other hand, trafficking at early endosomes in degradative sorting and endosomal recycling was attributed to PI4K2 $\alpha$ (Balla and Balla 2006). Interestingly, we found that similar to PI4K2 $\alpha$, PI4K2 $\beta$ colocalizes with the exocyst complex on endosomes, and that depletion of the $\beta$ isoform phenocopied loss of PI4K $2 \alpha$ regarding TfR miss-localization and exocytic trafficking defects. However, its colocalization with MTM1 
was less prominent compared to PI4K2 $\alpha$ (Figure 39a-e, compare to Figure 31). This could indicate that complex formation with MTM1 is specific for the $\alpha$ isoform. Nevertheless, we could not exclude functional redundancy between PI4K2 $\alpha$ and PI4K2 $\beta$. Future experiments will be needed to clarify potential differences between the type II PI4Ks in endosomal exocytosis. Possible scenarios include their interaction with and regulation by distinct SNARE complexes, active and inactive myotubularins. Alternatively, they might regulate different transport route such as fast, slow or late endosomal recycling as well as specialized recycling routes in cytokinesis, ciliogenesis or cell migration.

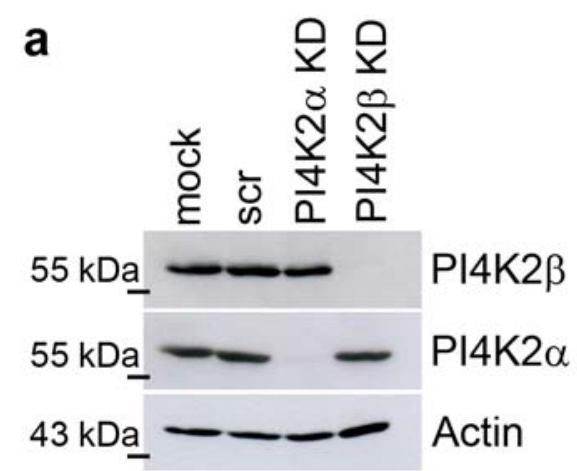

b

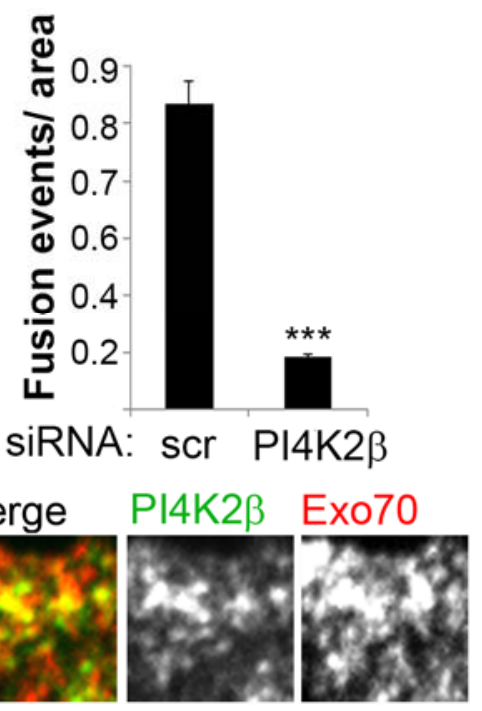

C
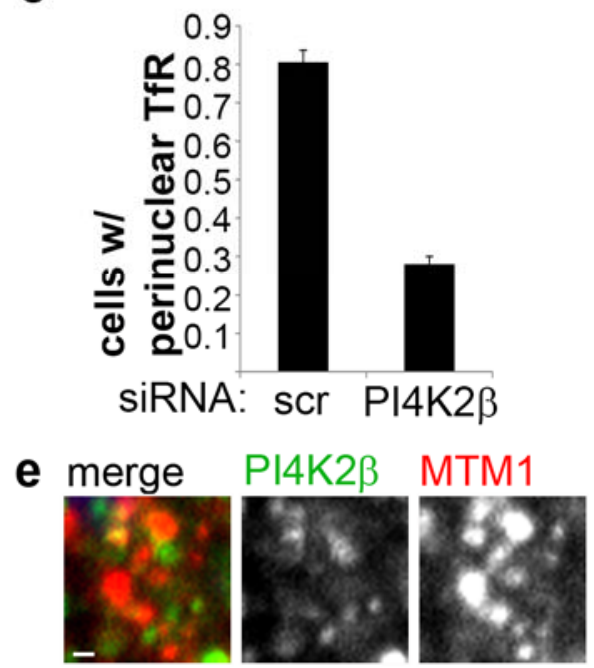

Figure 39: PI4K2 $\beta$ regulates endosomal exocytosis and exocyst function

(a) Efficient depletion of PI4K2 $\alpha$ and PI4K2 $\beta$ as demonstrated by immunoblotting of HeLa cell lysates. (b) Inhibition of Tf exocytosis in cells depleted of PI4K2 $\beta$ compared to scrambled (scr) siRNA-treated controls (Tf exocytic events/ area; mean \pm SEM, $n=3$ independent experiments with 5 videos analyzed per condition per experiment, ${ }^{* * *} P<0.001$, unpaired, two-tailed $t$-test). (c) Depletion of PI4K2 $\beta$ from HeLa cells causes TfR misslocalization. Normalized fraction of cells with perinuclear TfR was quantified (mean \pm SEM, $n=2$ independent experiments with 15-30 images analyzed per condition per experiment). (d) Colocalization of eGFP-PI4K2 $\beta$ and Exo70 in HeLa cells on endosomes, assessed by Exo70-specific antibody labeling. Representative images from one of 2 independent experiments are shown. Scale bar: $1 \mu \mathrm{m}$. (e) Colocalization of mCherry-MTM1 and eGFP-PI4K2 $\beta$ in HeLa cells on endosomes. Representative images from one of 2 independent experiments are shown. Scale bar: $1 \mu \mathrm{m}$. 
Interestingly, mutations in PI4K2 $\alpha$ are not associated with myopathies in humans. Furthermore, loss of PI4K2 $\alpha$ in mice leads to late onset neuronal degradation with no severe myopathy (Simons, Al-Shawi et al. 2009). This emphasizes the importance of PI4K2 $\alpha$ in vivo and indicates that loss of PI4K2 $\alpha$ can be compensated in most tissues. As discussed previously, PI4K2 $\beta$ would be ideally suited to substitute for PI4K2 $\alpha$. Alternatively, other PI4K2 $\alpha$-independent exit routes from the endosomal compartment could be favoured. However, these need to be defined I future experiments, but could comprise retrograde transport or recycling from late endosomes.

\subsubsection{PI(3)P turnover regulates endosomal tubulation and cargo sorting}

Tubulation of the early and recycling endosomal membrane is a further crucial part of endosomal maturation and cargo sorting. Cargo sorting via these tubules is coordinated by membrane deforming, PI(3)P-binding SNXs (van Weering, Verkade et al. 2012). Upon loss of MTM1, early and recycling endosomes accumulate PI(3)P and thereby PI(3)P-binding SNXs, i.e. SNX4 and SNX17, previously implicated in endosomal sorting (see Figure 3, introduction; Figure 18, results). Notably, MTM1 loss-of-function is at the endosomal level characterized by two distinct phenotypes (see Figure 12), which both lead to impaired endosomal exocytosis: 1) Depletion of perinuclear endosomes due to Kif16b-mediated outward transport of PI(3)P-containing endosomes and subsequent sub-plasmalemmal accumulation of fusion-deficient endosomes. 2) Depletion of perinuclear endosomes and extensive endosomal tubulation without an obvious accumulation in the cell periphery. Such a prolonged and strong tubulation is enabled by tubule decoration with and stabilization by PI(3)P-binding SNXs and microtubules (data not shown). Normally, endosomal tubules are transient, flexible and characterized by a high turnover rate. In MTM1 KDs, however, the molecular machinery required for tubule scission is presumably not assembled properly. This machinery could comprise of the Rab 11 family of interacting proteins (Rab11-FIP 1-5), EHD (Eps15-homology (EH) domain containing) proteins, which belong to the dynamin superfamily of GTPases, and/ or dynamins. A set of PI4K2 $\alpha$-dependent endosomal effector proteins localized to the tips of SNX-supported tubules upon loss of MTM1. These include EHD3 (Henmi, Morikawa et al. 2016), AP-3 (Craige, Salazar et al. 2008) and clathrin (data not shown). Although endosomal transport is intrinsically complex, these proteins might be essential for tubule scission, but require PI(3)P to PI(4)P turnover for their function.

Several lines of evidence suggest that manipulation of endosomal PI(3)P levels can cause extensive tubulation. First, targeted MTM1-mediated depletion of PI(3)P on Rab5- 
positive endosomes by a rapalogue-induced recruitment system led to the formation of microtubule-supported, endosomal tubules. At the same time recycling was impaired, while degradative sorting was only slightly affected (Fili, Calleja et al. 2006). Second, data from Carpentier et al. showed that class III PI3K inhibition causes endosomal swelling and impaired endosomal recycling, while release of PI3K inhibition induces endosomal tubulation, dynamin-dependent tubule scission and at the same time restores surface delivery of recycling cargo (Carpentier, N'Kuli et al. 2013). Third, recent results from Munson et al. using the Vps34-specific inhibitor VPS34-IN1 identified a late endosomal and lysosomal PI(3)P pool, regulated by Vps34. Acute inhibition of Vps34 led to extensive lysosomal tubule formation (Munson, Allen et al. 2015), a process implicated in autophagosome-lysosome reformation (ALR).

Together with our observations, these results indicate that PI(3)P-turnover is essential for endosomal tubulation, maturation and recycling from early as well as late endosomes and lysosomes in ALR. Defective PI(3)P-generation and PI(3)P-depletion cause morphological changes of the endosomal system. This suggests that spatiotemporal regulation of PI(3)P is crucial for the sequential recruitment of PI(3)P-binding adaptor proteins, subsequent cargo sorting and dissociation of these PI(3)P-specific adaptors upon depletion of PI(3)P.

\section{2 $\mathrm{PI}(3) \mathrm{P}-$ to-PI(4)P conversion regulates endosomal recycling}

\subsubsection{PI conversion in endosomal exocytosis parallels switching of the Rab identity}

Progression through the endosomal membrane system crucially depends on conversion of membrane identity defined by PIs and small Rab GTPases. Thus, we experimentally addressed the PI identity of exocytic vesicles (see Figure 28). PI(3)P-enriched endosomes, marked by the 2xFYVE probe, were unable to undergo exocytosis, while endosomes devoid of PI(3)P acquired PI(4)P prior to fusion. Furthermore, artificial elevation of PI(4)P failed to rescue MTM1-dependent exocytic defects, suggesting that PI(3)P removal is a prerequisite for PI(4)P acquisition. As the 2xFYVE-probe binds endosomal PI(3)P with a very high affinity considerably stronger than endogenous FYVE-domain containing proteins -, PI(3)P-positive endosomes masked by the 2xFYVE probe were stalled and immobile. Thus, expression of the 2xFYVE-probe mimicked the endosomal accumulation of PI(3)P-binding effector proteins seen upon loss of MTM1. This blocks accessibility of PI(3)P for PI(3)P-dependent motor 
proteins and PI(3)P metabolizing enzymes (Petiot, Faure et al. 2003). To allow dissociation of the FYVE-probe from endosomes, a single FYVE domain under a weak promoter (Hayakawa, Hayes et al. 2004; Stuffers, Malerod et al. 2010) or knockin of the GFP-tagged FYVE domain using CRISPR/ Cas9 technology within a genomic safe harbour locus (Doudna and Charpentier 2014) should be used. Thus, it would match binding constants of endogenous proteins. This would allow us to address the time course of endosomal PI(3)P-acquisition, its loss during exocytosis as well as to monitor subsequent acquisition of PI(4)P.

Studies by others hinted at additional PI species involved in secretion and exocytosis: $\mathrm{PI}(3) \mathrm{P}$, synthesized by $\mathrm{PI} 3 \mathrm{KC} 2 \alpha$ on chromaffin granules, positively regulated secretion (Meunier, Osborne et al. 2005; Wen, Osborne et al. 2008). However, PI(3)P turnover and/ or PI conversion was not addressed by this study. $\mathrm{PI}(4,5) \mathrm{P}_{2}$ stimulates synaptic vesicle exocytosis and exocyst-dependent, SNARE-mediated PM fusion, reviewed in (Ammar, Kassas et al. 2013). However, these studies support a PI(4,5) $\mathrm{P}_{2}$ function at the $\mathrm{PM}$ without clear evidence of a vesicular $\mathrm{PI}(4,5) \mathrm{P}_{2}$ pool crucial for $\mathrm{PM}$ fusion. In the present study, neither $\mathrm{PI}(3) \mathrm{P}, \mathrm{PI}(3,4) \mathrm{P}_{2}$ nor $\mathrm{PI}(4,5) \mathrm{P}_{2}$ were detectable on endosomes that were in the process of fusing with the PM.

Taken together, these data indicate that PI(3)P-positive exocytic vesicles loose their $\mathrm{PI}(3) \mathrm{P}$-identity and subsequently acquire PI(4)P prior to exocytosis. While this is required to match the PI content of the PM, exocytic sites at the PM are predefined by local PI(4,5) $\mathrm{P}_{2}$ synthesis.

According to the PI content, early Rab5-positive endosomes do not exocytose, but need to loose Rab5 and acquire Rab11 to become fusion competent (see Figure 33). Furthermore, exocytic vesicles approaching the PM are PI(4)P- and Rab11-positive. Rab11 dissociates from endosomes shortly before while PI(4)P dispersion coincides with PM fusion. As no major PI species has been assigned to Rab11-positive recycling endosomes so far (Jean and Kiger 2012), this is of particular importance. Previous data from D. melanogaster and yeast corroborate our data: Exocytic traffic to the midbody in cytokinesis requires PI(4)P on Rab11-positive recycling endosomes, shown in D.melanogaster (Polevoy, Wei et al. 2009). In yeast, trafficking from the TGN en route to the PM is regulated by PI(4)P levels on Rab11positive secretory vesicles (Mizuno-Yamasaki, Medkova et al. 2010). This implies PI(4)P as the major lipid requirement on vesicles for outward transport and PM fusion. 


\subsubsection{Regulation of the exocyst complex by endosomal and PM determinants}

Exocytic sites are defined by PIP5KI-mediated $\mathrm{PI}(4,5) \mathrm{P}_{2}$ synthesis and $\mathrm{PI}(4,5) \mathrm{P}_{2}-$ dependent recruitment of the exocyst (Exo70 and Sec3) to the PM (Thapa, Sun et al. 2012; Xiong, $\mathrm{Xu}$ et al. 2012). Tethering exocytic vesicles, carrying the remaining exocyst subunits, to these predefined sites primes them for SNARE-mediated PM fusion. However, lipid and protein requirements for targeting the exocyst to exocytic vesicles are less well understood. In extension of previous studies, which reported a $\mathrm{PI}(4,5) \mathrm{P}_{2}$-dependent Exo70 localization to the $\mathrm{PM}$ ( $\mathrm{He}$, Xi et al. 2007), our data indicate the existence of a vesicular PI(4)P-dependent Exo70 pool. Further, recruitment to vesicles requires Rab11, MTM1 and PI4K2 $\alpha$ (see Figure 32, 34), in line with previous data (Ortiz, Medkova et al. 2002; Zhang, Ellis et al. 2004). Thus, exocyst assembly seems to be coupled to PI(3)P-depletion, PI(4)P-synthesis and the switch in Rab5-to-Rab11 identity. In addition, SNARE composition contributes to recruitment of the exocyst complex (Kubo, Kobayashi et al. 2015). Vamp2 i.e. targets the exocyst to recycling endosomes. Interestingly, Exo70 binds $\mathrm{PI}(3,5) \mathrm{P}_{2}$ and $\mathrm{PI}(4,5) \mathrm{P}_{2}$ with comparable affinities (He, Xi et al. 2007). The exocyst complex is further associated with Vamp7-positive, $\mathrm{PI}(3,5) \mathrm{P}_{2}$-enriched late endosomes and involved in late endosomal recycling (see section 4.3.1). Thus, coincidence detection of at least two of these components, i.e. SNAREs, PIs, Rabs and adaptor proteins, is required to define endosomal subdomains for exocytic traffic, efficient assembly of the exocyst complex and provide specificity to its targeting. Thereby, diverse cellular routes that require outward transport, tethering and PM fusion might be distinguished. These comprise ciliogenesis, cytokinesis, cell migration, polarization and tight junction formation (Heider and Munson 2012). Although the exocyst complex is required, composition of the exocyst complex might differ in all of them. Further, protein and lipid requirements are poorly understood and have to be determined in future studies.

\subsection{Common features of MTM1 deficiency cause XLCNM pathologies}

The MTM1- and PI4K2 $\alpha$-mediated PI conversion mechanism unraveled in the present study is essential for membrane remodeling in fibroblasts from XLCNM patients (Figure 40, (1)). Exocytic membrane traffic of integrin-based adhesion complexes is especially required for muscle attachment to the extracellular matrix and to tendons. A second key complex in muscle attachment, the Dystroglycan adhesion complex, remains however unaffected 
(Ribeiro, Yuan et al. 2011). This implicates that MTM1 selectively affects integrin traffic or that other adhesion complexes do not undergo frequent endo-exocytic cycles during muscle development and maintenance. Muscle fibers from XLCNM patients further display abnormal T-tubule morphology, sarcoplasmic reticulum (SR) dilatation and triads dysorganisation. Thus, impaired excitation-contraction coupling, observed in these patients, might be a secondary cause of defective membrane organization. Herein, the major calcium release channel of the SR, the ryanodine receptor (Ryr1), is coupled to the voltage-sensitive dihydropyridine receptor (DHPR) in the T-tubule. Thus, $\mathrm{Ca}^{2+}$ release from the sarcolemma is initiated by excitation. Additionally, local PI homeostasis of $\mathrm{PI}(3) \mathrm{P}$ and $\mathrm{PI}(3,5) \mathrm{P}_{2}$, controlled by myotubularins, was shown to regulate ion channel activity (Srivastava, Li et al. 2005; Shen, $\mathrm{Yu}$ et al. 2009). Defective membrane organization and channel activity might have additive effects on muscle dysfunction, reflected by the severe form of myotubular myopathy.

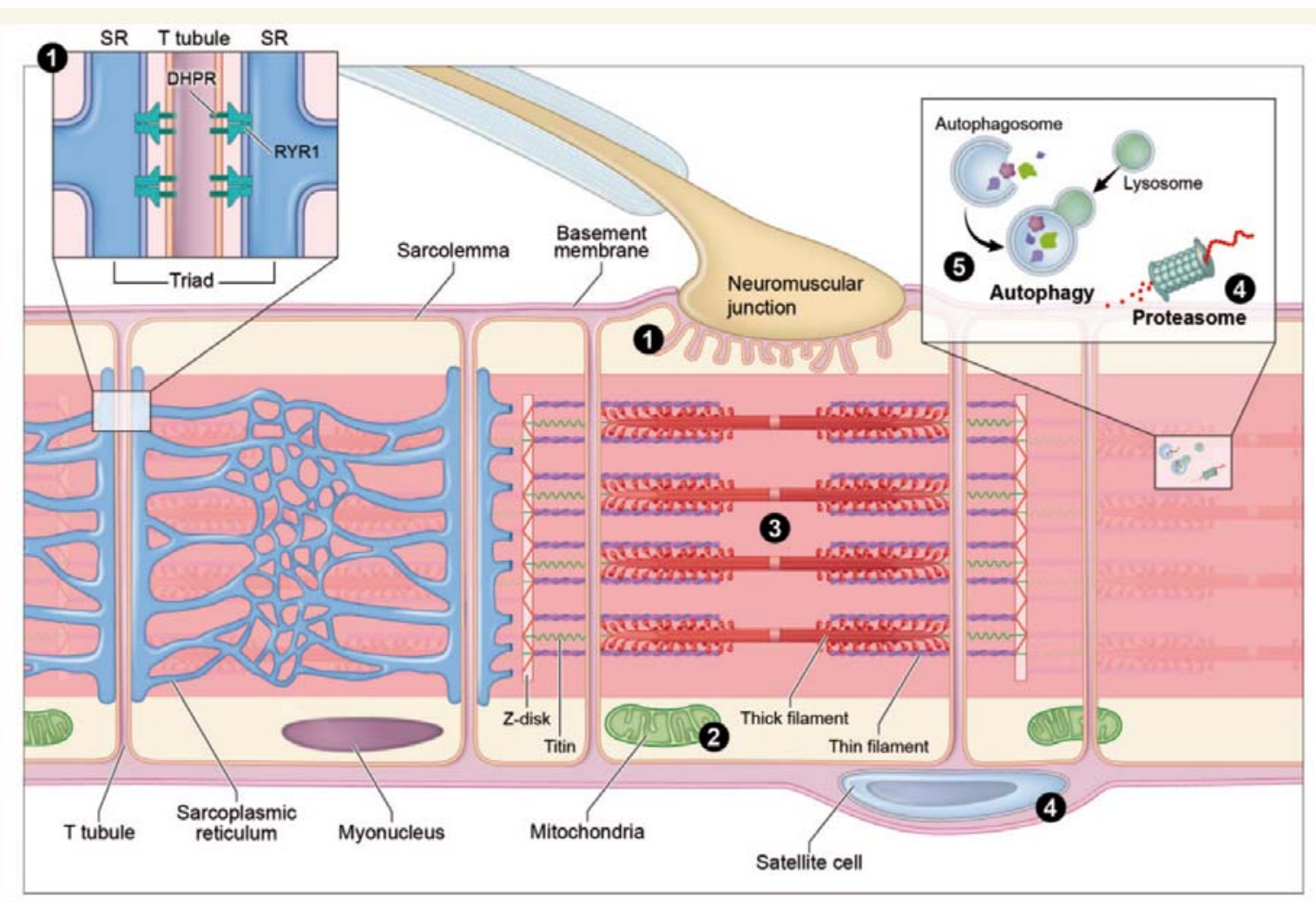

Figure 40: Schematic representation of a muscle fiber

The pathophysiological concepts are placed in their cellular context: (1) sarcolemmal and intracellular membrane remodelling and excitation-contraction coupling; (2) mitochondrial distribution and function; (3) myofibrillar force generation; (4) atrophy; and (5) autophagy. DHPR - dihydropyridine receptor; RYR1 - ryanodine receptor; SR - sarcoplasmic reticulum (Ravenscroft, Laing et al. 2015).

In addition to these defects (see Figure 40, (1)), the pathophysiology of XLCNM patients include: (2) mitochondrial redistribution and dysfunction; (3) defects in myofibrillar 
force generation; (4) atrophy; and (5) defects in autophagy induction and autophagic flux (Figure 40) (Ravenscroft, Laing et al. 2015). Notably, genetic defects in MTM1 cause 4 (1, 2, 4 and 5) out of 5 of these features. With regard to (2), it is interesting to note that loss of function mutations in desmin cause altered desmin intermediate filaments (IF) and lead to desmin-related myophathy (DRM) (Omary, Coulombe et al. 2004). Characteristic for DRM are mitochondrial dysfunction and abnormal distribution. MTM1 directly regulates desmin IFs in vitro and in vivo. Thus, XLCNM-causing mutations in the desmin-binding interface of MTM1 abolish MTM1-desmin complex formation leading to pathological features reminiscent of DRM (Hnia, Tronchere et al. 2011).

In autophagy (Figure 40, (5)), engulfment of cytosolic material or organelles by the isolation membrane initiates autophagy and forms the autophagosome. Autophagosomes subsequently fuse with lysosomes for cargo degradation. The isolation membrane is enriched in $\mathrm{PI}(3) \mathrm{P}$ and $\mathrm{PI}(3) \mathrm{P}$-effector proteins. However, accumulation of $\mathrm{PI}(3) \mathrm{P}$-effectors on the isolation membrane prevents its closure. To dissociate these proteins from the isolation membrane prior to fusion with lysosomes, $\mathrm{PI}(3) \mathrm{P}$ dephosphorylation is required, in yeast by the myotubularin Ymr1, in C. elegans by MTM-3 (Cebollero, van der Vaart et al. 2012; Wu, Cheng et al. 2014). This surprisingly parallels exocytic traffic where MTM1-dependent PI(3)P depletion is needed to dissociate PI(3)P-binding SNXs from endosomes prior to fusion with the PM.

XLCNM pathology is inherently complex due to various possible combinations of defects attributed to the MTM1 phosphatase-dependent (i.e. 1 and 5) and phosphataseindependent functions (i.e. 2). Nevertheless, common underlying mechanisms seem to dictate its enzymatic role. In this study, we were able to reverse trafficking defects in XLCNM patient fibroblasts by pharmacological inhibition of its enzymatic counterpart Vps34. It is tempting to assume that identify XLCNM mutations that selectively affect the enzymatic activity of MTM1 might allow us to combat this devastating disease by VPS34-IN1 treatment.

\subsubsection{Late endosomal recycling contributes to $\beta 1$-integrin traffic}

Muscle fibers as well as fibroblasts from XLCNM patients accumulate endosomal cargo such as TfR and integrins within PI(3)P-enriched recycling endosomes (see Figure 8) (Ribeiro, Yuan et al. 2011). In addition, integrins accumulate in late endosomes devoid of TfR. This might indicate that cargo exit from endosomes en route to late endosomes is used as an escape route for integrins to prevent cargo accumulation and trapping in early and recycling endosomes. Indeed, alternative to Rab4- or Rab11-dependent recycling - the major 
sorting routes, which integrins share with endosomal cargo such as TfR-, they can be recycled from late endosomes and lysosomes (Rainero and Norman 2013). Notably, myotubularins, i.e. MTM1 and MTMR2, localize to late endosomes as well. Thus, loss of MTM1 in XLCNM patients presumably impairs conventional as well as late endosomal recycling of integrins en route to the PM.

The discrimination between different integrin-transport routes highly depends on ligand binding as well as the conformation of the $\alpha$ - and $\beta$-integrin heterodimer. While inactive integrins traffic via a fast Rab4-dependent recycling route, integrins recycle with slower kinetics in their active conformation via Rab11-recycling endosomes (Arjonen, Alanko et al. 2012). SNX17, a SNX also crucial for TfR recycling, binds PI(3)P and the NPxYsorting motif in the cytosolic tail of $\beta 1$-integrin at early endosomes. Thus, it is essential for their recycling and thereby prevents degradative sorting (Bottcher, Stremmel et al. 2012; Steinberg, Heesom et al. 2012). However, loss of SNX17 and upregulation of the Rab11-like GTPase Rab25 leads to the sorting of active-conformation and ligand-occupied integrins to late endosomes (Dozynkiewicz, Jamieson et al. 2012). Late endosomal enrichment of chloride intracellular channel 3 (CLIC3) prevents integrin degradation, presumably by interfering with inward budding from the limiting membrane and ILV generation, or by interfering with ubiquitylation. Further, CLIC3 is found on tubules emanating from late endosomes and lysosomes (Rainero and Norman 2013). Here, PI(4)P and PI(4,5) $\mathrm{P}_{2}$ are essential for tubule formation from predominantly $\mathrm{PI}(3) \mathrm{P}$ - and $\mathrm{PI}(3,5) \mathrm{P}_{2}$-positive late endosomes (Ktistakis and Tooze 2013; Hammond, Machner et al. 2014). Further, vesicular exit and late endosomal recycling requires VAMP7 and the exocyst complex. Interestingly, PI4K2 $\alpha$ associates with VAMP7 and might thus be suited to produce such a late endosomal PI(4)P pool. It is tempting to speculate that recycling from late endosomes requires formation of tubular cargo-enriched subdomains, $\mathrm{PI}(3) \mathrm{P} / \mathrm{PI}(3,5) \mathrm{P}_{2}$-to-PI(4)P conversion mediated by $\mathrm{PI} 4 \mathrm{~K} 2 \alpha$ and MTM1 and subsequent exocyst-dependent fusion with the PM. Vamp7, local enrichment of $\mathrm{PI}(3,5) \mathrm{P}_{2}$, CLIC3 and Rab25 distinguish late endosomes from early and recycling endosomes. However, late endosomal $\beta 1$-integrin accumulations were not seen upon depletion of PI4K2 $\alpha$ (data not shown). Thus, late endosomal recycling might involve a different subset of 4'- and 3'metabolising enzymes, i.e. the lysosomal PI4K3 $\beta$ (Sridhar, Patel et al. 2013) and MTMR2. Future experiments have to clarify the spatiotemporal regulation of PI and Rab conversion in late endosomal recycling. 


\subsection{Outlook}

The identification of a PI conversion mechanism that is coupled to a switch in Rab identity and regulates exit from endosomes contributes significantly to our understanding of the spatiotemporal regulation of intracellular membrane traffic. However, most of the existing data, including the present study, concentrates on MTM1 and MTMR2, while other active myotubularins remain poorly characterized. To deepen our understanding of this 3phosphatase family is especially important as a wide range of diseases are caused by loss of myotubularins. These range from spermatogenesis (MTMR2 and MTMR5) to neuropathies (CMT4B1, MTMR2 and CMT4B2, MTMR13) and myophathies (MTM1 and MTMR1). Furthermore, as our study identified Vps34-inhibition as a potential avenue to combat MTM1dependent XLCNM in humans, it should be clarified how and if this inhibitor could be applied to in vivo studies and to patients.

It remains to be clarified how targeting of these enzymes to endosomal subpopulations or subdomains within the same endosome might be achieved. Do myotubularins, i.e. MTM1, MTMR2 and MTMR4, regulate distinct exit routes from the early endosome such as early-tolate endosomal progression, retrograde endosome-to-TGN transport and/ or fast and slow recycling? Are these endosomal subdomains defined by specific Rab GTPases, PI composition and/ or additional effector proteins? The answer might partially lie in their regulation and localization by inactive MTMRs. Defined 'active/dead' combinations could regulate sub-compartmental targeting and fine-tune enzymatic activity and/ or substrate specificity. For example, MTMR12 associates with MTM1 and MTMR2. MTMR2 further binds inactive MTMR5 and MTMR13, while MTM1 can also interact with inactive MTMR10. Tissue specific expression patterns or distinct developmental regulation might account for the huge number of active and inactive myotubularins. An additional layer of complexity is added by their association with class II and III PI3Ks, and by their reciprocal regulation in the control of endosomal PI turnover and homeostasis. Another debated question is PI binding specificities, enzymatic activities and substrate preference of myotubularins, including MTM1. In addition to $\mathrm{PI}(3) \mathrm{P}$ and $\mathrm{PI}(3,5) \mathrm{P}_{2}$, early studies indicated that myotubularins can accept PI(3,4) $\mathrm{P}_{2}$ (Taylor, Maehama et al. 2000; Kim, Taylor et al. 2002) or $\mathrm{I}(1,3) \mathrm{P}_{2}$ (Mochizuki and Majerus 2003) as substrates. Systematic analysis of these enzyme properties might add to understanding their differences as well as potential redundancies.

Beside endosomal exocytosis, various endosomal trafficking routes are regulated by more than one PI species. This suggests that they require the concerted action of PI kinases and 
phosphatases, their lipid substrates and thereby protein-lipid effector complexes. PI 3phosphates are a hallmark of endosomal membranes. Thus it is conceivable to assume that myotubularins are essential players in most trafficking routes emanating from endosomes. For example, a module comprising MTM1/ MTMR2, PI4K2 $\alpha$ and PIKfyve could be targeted to late endosomes via $\mathrm{PI}(3) \mathrm{P} / \mathrm{PI}(3,5) \mathrm{P}_{2}$, Vamp7 and Rab25, mediate $\mathrm{PI}(3) \mathrm{P} / \mathrm{PI}(3,5) \mathrm{P}_{2}$-to- $\mathrm{PI}(4) \mathrm{P}$ conversion, allow tubule-based recycling from late endosomes and exocyst-dependent fusion with the PM (as discussed in section 4.3.1) (Rainero and Norman 2013). In ciliogenesis PI3KC2 $\alpha$, a known interactor of MTMR13, synthesizes a PI(3)P pool on Rab11-recycling endosomes that is required for Rab11-to-Rab8 conversion and exocyst-dependent membrane addition at the base of the primary cilium (Franco, Gulluni et al. 2014). Cytokinesis, on the other hand, requires PI(3)P- as well as PI(4)P-effectors, Rab11-endosomes and exocystmediated membrane delivery to the midbody (Brill, Wong et al. 2011; Schink, Raiborg et al. 2013). Other pathways that are regulated by multiple PI species, Rab-conversion and/ or the exocyst complex include polarized transport and adhesion complex formation in epithelial cells, cell migration and ALR. This suggests PI as well as Rab conversion as a common overarching principle. Future research has to delineate the role of 3'- and 4'-metabolising enzymes, the spatiotemporal control of Rab and PI conversion and the effector complexes involved. 


\section{Bibliography}

Al-Qusairi, L., I. Prokic, et al. (2013). "Lack of myotubularin (MTM1) leads to muscle hypotrophy through unbalanced regulation of the autophagy and ubiquitin-proteasome pathways." FASEB J 27(8): 3384-3394.

Ammar, M. R., N. Kassas, et al. (2013). "Lipids in Regulated Exocytosis: What are They Doing?" Front Endocrinol (Lausanne) 4: 125.

Amoasii, L., K. Hnia, et al. (2012). "Myotubularin phosphoinositide phosphatases in human diseases." Curr Top Microbiol Immunol 362: 209-233.

Arjonen, A., J. Alanko, et al. (2012). "Distinct recycling of active and inactive betal integrins." Traffic 13(4): 610-625.

Axelrod, D., T. P. Burghardt, et al. (1984). "Total internal reflection fluorescence." Annu Rev Biophys Bioeng 13: 247-268.

Bago, R., N. Malik, et al. (2014). "Characterization of VPS34-IN1, a selective inhibitor of Vps34, reveals that the phosphatidylinositol 3-phosphate-binding SGK3 protein kinase is a downstream target of class III phosphoinositide 3-kinase." Biochem J 463(3): 413-427.

Balla, A. and T. Balla (2006). "Phosphatidylinositol 4-kinases: old enzymes with emerging functions." Trends Cell Biol 16(7): 351-361.

Balla, A., G. Tuymetova, et al. (2002). "Characterization of type II phosphatidylinositol 4kinase isoforms reveals association of the enzymes with endosomal vesicular compartments." J Biol Chem 277(22): 20041-20050.

Balla, A., G. Tuymetova, et al. (2005). "A plasma membrane pool of phosphatidylinositol 4phosphate is generated by phosphatidylinositol 4-kinase type-III alpha: studies with the PH domains of the oxysterol binding protein and FAPP1." Mol Biol Cell 16(3): 1282-1295.

Balla, T. (2013). "Phosphoinositides: tiny lipids with giant impact on cell regulation." Physiol

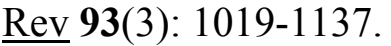

Balla, T. (2016). "Cell biology: Lipid code for membrane recycling." Nature.

Barylko, B., Y. S. Mao, et al. (2009). "Palmitoylation controls the catalytic activity and subcellular distribution of phosphatidylinositol 4-kinase II \{alpha\}." J Biol Chem 284(15): 9994-10003. 
Begley, M. J., G. S. Taylor, et al. (2003). "Crystal structure of a phosphoinositide phosphatase, MTMR2: insights into myotubular myopathy and Charcot-Marie-Tooth syndrome." Mol Cell 12(6): 1391-1402.

Behnia, R. and S. Munro (2005). "Organelle identity and the signposts for membrane traffic." Nature 438(7068): 597-604.

Berger, P., I. Berger, et al. (2006). "Multi-level regulation of myotubularin-related protein-2 phosphatase activity by myotubularin-related protein-13/set-binding factor-2." Hum Mol Genet 15(4): 569-579.

Blondeau, F., J. Laporte, et al. (2000). "Myotubularin, a phosphatase deficient in myotubular myopathy, acts on phosphatidylinositol 3-kinase and phosphatidylinositol 3-phosphate pathway." Hum Mol Genet 9(15): 2223-2229.

Blumental-Perry, A., C. J. Haney, et al. (2006). "Phosphatidylinositol 4-phosphate formation at ER exit sites regulates ER export." Dev Cell 11(5): 671-682.

Bojjireddy, N., J. Botyanszki, et al. (2014). "Pharmacological and genetic targeting of the PI4KA enzyme reveals its important role in maintaining plasma membrane phosphatidylinositol 4-phosphate and phosphatidylinositol 4,5-bisphosphate levels." J Biol Chem 289(9): 6120-6132.

Bolino, A., A. Bolis, et al. (2004). "Disruption of Mtmr2 produces CMT4B1-like neuropathy with myelin outfolding and impaired spermatogenesis." J Cell Biol 167(4): 711-721.

Bolis, A., S. Coviello, et al. (2009). "Dlg1, Sec8, and Mtmr2 regulate membrane homeostasis in Schwann cell myelination." J Neurosci 29(27): 8858-8870.

Bonifacino, J. S. and J. H. Hurley (2008). "Retromer." Curr Opin Cell Biol 20(4): 427-436.

Bottcher, R. T., C. Stremmel, et al. (2012). "Sorting nexin 17 prevents lysosomal degradation of betal integrins by binding to the beta1-integrin tail." Nat Cell Biol 14(6): 584-592.

Boyd, C., T. Hughes, et al. (2004). "Vesicles carry most exocyst subunits to exocytic sites marked by the remaining two subunits, Sec3p and Exo70p." J Cell Biol 167(5): 889-901.

Bradford, M. M. (1976). "A rapid and sensitive method for the quantitation of microgram quantities of protein utilizing the principle of protein-dye binding." Anal Biochem 72: 248254.

Brill, J. A., R. Wong, et al. (2011). "Phosphoinositide function in cytokinesis." Curr Biol 21(22): R930-934.

Buj-Bello, A., D. Furling, et al. (2002). "Muscle-specific alternative splicing of myotubularinrelated 1 gene is impaired in DM1 muscle cells." Hum Mol Genet 11(19): 2297-2307. 
Buj-Bello, A., V. Laugel, et al. (2002). "The lipid phosphatase myotubularin is essential for skeletal muscle maintenance but not for myogenesis in mice." Proc Natl Acad Sci U S A 99(23): 15060-15065.

Burden, J. J., X. M. Sun, et al. (2004). "Sorting motifs in the intracellular domain of the low density lipoprotein receptor interact with a novel domain of sorting nexin-17." J Biol Chem 279(16): 16237-16245.

Cao, C., J. M. Backer, et al. (2008). "Sequential actions of myotubularin lipid phosphatases regulate endosomal PI(3)P and growth factor receptor trafficking." Mol Biol Cell 19(8): 33343346.

Cao, C., J. Laporte, et al. (2007). "Myotubularin lipid phosphatase binds the hVPS15/hVPS34 lipid kinase complex on endosomes." Traffic 8(8): 1052-1067.

Carlton, J., M. Bujny, et al. (2004). "Sorting nexin-1 mediates tubular endosome-to-TGN transport through coincidence sensing of high- curvature membranes and 3phosphoinositides." Curr Biol 14(20): 1791-1800.

Carpentier, S., F. N'Kuli, et al. (2013). "Class III phosphoinositide 3-kinase/VPS34 and dynamin are critical for apical endocytic recycling." Traffic 14(8): 933-948.

Cebollero, E., A. van der Vaart, et al. (2012). "Phosphatidylinositol-3-phosphate clearance plays a key role in autophagosome completion." Curr Biol 22(17): 1545-1553.

Christoforidis, S., M. Miaczynska, et al. (1999). "Phosphatidylinositol-3-OH kinases are Rab5 effectors." Nat Cell Biol 1(4): 249-252.

Ciechanover, A., A. L. Schwartz, et al. (1983). "Kinetics of internalization and recycling of transferrin and the transferrin receptor in a human hepatoma cell line. Effect of lysosomotropic agents." J Biol Chem 258(16): 9681-9689.

Craige, B., G. Salazar, et al. (2008). "Phosphatidylinositol-4-kinase type II alpha contains an AP-3-sorting motif and a kinase domain that are both required for endosome traffic." Mol Biol Cell 19(4): 1415-1426.

Cullen, P. J. (2008). "Endosomal sorting and signalling: an emerging role for sorting nexins." Nat Rev Mol Cell Biol 9(7): 574-582.

Czogalla, A., M. Grzybek, et al. (2014). "Validity and applicability of membrane model systems for studying interactions of peripheral membrane proteins with lipids." Biochim Biophys Acta 1841(8): 1049-1059.

Czubayko, M., P. Knauth, et al. (2006). "Sorting nexin 17, a non-self-assembling and a PtdIns(3)P high class affinity protein, interacts with the cerebral cavernous malformation related protein KRIT1." Biochem Biophys Res Commun 345(3): 1264-1272. 
Dall'Armi, C., K. A. Devereaux, et al. (2013). "The role of lipids in the control of autophagy." Curr Biol 23(1): R33-45.

Danson, C., E. Brown, et al. (2013). "SNX15 links clathrin endocytosis to the PtdIns3P early endosome independently of the APPL1 endosome." J Cell Sci 126(Pt 21): 4885-4899.

de Lartigue, J., H. Polson, et al. (2009). "PIKfyve regulation of endosome-linked pathways." Traffic 10(7): 883-893.

De Matteis, M. A. and A. Godi (2004). "PI-loting membrane traffic." Nat Cell Biol 6(6): 487492.

de Renzis, S., B. Sonnichsen, et al. (2002). "Divalent Rab effectors regulate the subcompartmental organization and sorting of early endosomes." Nat Cell Biol 4(2): 124-133.

Devereaux, K., C. Dall'Armi, et al. (2013). "Regulation of mammalian autophagy by class II and III PI 3-kinases through PI3P synthesis." PLoS One 8(10): e76405.

Di Paolo, G. and P. De Camilli (2006). "Phosphoinositides in cell regulation and membrane dynamics." Nature 443(7112): 651-657.

Donoso, M., J. Cancino, et al. (2009). "Polarized traffic of LRP1 involves AP1B and SNX17 operating on Y-dependent sorting motifs in different pathways." Mol Biol Cell 20(1): 481497.

Doudna, J. A. and E. Charpentier (2014). "Genome editing. The new frontier of genome engineering with CRISPR-Cas9." Science 346(6213): 1258096.

Dowling, J. J., A. P. Vreede, et al. (2009). "Loss of myotubularin function results in T-tubule disorganization in zebrafish and human myotubular myopathy." PLoS Genet 5(2): e1000372.

Dozynkiewicz, M. A., N. B. Jamieson, et al. (2012). "Rab25 and CLIC3 collaborate to promote integrin recycling from late endosomes/lysosomes and drive cancer progression." Dev Cell 22(1): 131-145.

Dubuke, M. L., S. Maniatis, et al. (2015). "The Exocyst Subunit Sec6 Interacts with Assembled Exocytic SNARE Complexes." J Biol Chem 290(47): 28245-28256.

Dyve, A. B., J. Bergan, et al. (2009). "Sorting nexin 8 regulates endosome-to-Golgi transport." Biochem Biophys Res Commun 390(1): 109-114.

Erdmann, K. S., Y. Mao, et al. (2007). "A role of the Lowe syndrome protein OCRL in early steps of the endocytic pathway." Dev Cell 13(3): 377-390.

Felgner, P. L., T. R. Gadek, et al. (1987). "Lipofection: a highly efficient, lipid-mediated DNA-transfection procedure." Proc Natl Acad Sci U S A 84(21): 7413-7417. 
Fetalvero, K. M., Y. Yu, et al. (2013). "Defective autophagy and mTORC1 signaling in myotubularin null mice." Mol Cell Biol 33(1): 98-110.

Fili, N., V. Calleja, et al. (2006). "Compartmental signal modulation: Endosomal phosphatidylinositol 3-phosphate controls endosome morphology and selective cargo sorting." Proc Natl Acad Sci U S A 103(42): 15473-15478.

Franco, I., F. Gulluni, et al. (2014). "PI3K class II alpha controls spatially restricted endosomal PtdIns3P and Rab11 activation to promote primary cilium function." Dev Cell 28(6): 647-658.

Franklin, N. E., G. S. Taylor, et al. (2011). "Endosomal targeting of the phosphoinositide 3phosphatase MTMR2 is regulated by an N-terminal phosphorylation site." J Biol Chem 286(18): 15841-15853.

Galli, T., T. Chilcote, et al. (1994). "Tetanus toxin-mediated cleavage of cellubrevin impairs exocytosis of transferrin receptor-containing vesicles in CHO cells." J Cell Biol 125(5): 10151024.

Gary, J. D., A. E. Wurmser, et al. (1998). "Fab1p is essential for PtdIns(3)P 5-kinase activity and the maintenance of vacuolar size and membrane homeostasis." J Cell Biol 143(1): 65-79.

Godi, A., P. Pertile, et al. (1999). "ARF mediates recruitment of PtdIns-4-OH kinase-beta and stimulates synthesis of PtdIns(4,5)P2 on the Golgi complex." Nat Cell Biol 1(5): 280-287.

Gordon, D. E., L. M. Bond, et al. (2010). "A targeted siRNA screen to identify SNAREs required for constitutive secretion in mammalian cells." Traffic 11(9): 1191-1204.

Guo, J., M. R. Wenk, et al. (2003). "Phosphatidylinositol 4-kinase type IIalpha is responsible for the phosphatidylinositol 4-kinase activity associated with synaptic vesicles." Proc Natl Acad Sci U S A 100(7): 3995-4000.

Hammond, G. R., M. J. Fischer, et al. (2012). "PI4P and PI(4,5)P2 are essential but independent lipid determinants of membrane identity." Science 337(6095): 727-730.

Hammond, G. R., M. P. Machner, et al. (2014). "A novel probe for phosphatidylinositol 4phosphate reveals multiple pools beyond the Golgi." J Cell Biol 205(1): 113-126.

Hammond, G. R., G. Schiavo, et al. (2009). "Immunocytochemical techniques reveal multiple, distinct cellular pools of PtdIns4P and PtdIns(4,5)P(2)." Biochem J 422(1): 23-35.

Haugsten, E. M., A. Oppelt, et al. (2013). "Phosphatidylinositol 5-phosphate is a second messenger important for cell migration." Commun Integr Biol 6(5): e25446.

Hayakawa, A., S. J. Hayes, et al. (2004). "Structural basis for endosomal targeting by FYVE domains." J Biol Chem 279(7): 5958-5966. 
He, B., F. Xi, et al. (2007). "Exo70 interacts with phospholipids and mediates the targeting of the exocyst to the plasma membrane." EMBO J 26(18): 4053-4065.

Heider, M. R. and M. Munson (2012). "Exorcising the exocyst complex." Traffic 13(7): 898907.

Henmi, Y., Y. Morikawa, et al. (2016). "PtdIns4KIIalpha generates endosomal PtdIns(4)P and is required for receptor sorting at early endosomes." Mol Biol Cell.

Herman, G. E., M. Finegold, et al. (1999). "Medical complications in long-term survivors with X-linked myotubular myopathy." J Pediatr 134(2): 206-214.

Hertzog, M. and P. Chavrier (2011). "Cell polarity during motile processes: keeping on track with the exocyst complex." Biochem J 433(3): 403-409.

Hnia, K., H. Tronchere, et al. (2011). "Myotubularin controls desmin intermediate filament architecture and mitochondrial dynamics in human and mouse skeletal muscle." J Clin Invest 121(1): $70-85$.

Hnia, K., I. Vaccari, et al. (2012). "Myotubularin phosphoinositide phosphatases: cellular functions and disease pathophysiology." Trends Mol Med 18(6): 317-327.

Hoepfner, S., F. Severin, et al. (2005). "Modulation of receptor recycling and degradation by the endosomal kinesin KIF16B." Cell 121(3): 437-450.

Hsu, F., F. Hu, et al. (2015). "Spatiotemporal control of phosphatidylinositol 4-phosphate by Sac2 regulates endocytic recycling." J Cell Biol 209(1): 97-110.

Hsu, V. W., M. Bai, et al. (2012). "Getting active: protein sorting in endocytic recycling." Nat Rev Mol Cell Biol 13(5): 323-328.

Hsu, V. W. and R. Prekeris (2010). "Transport at the recycling endosome." Curr Opin Cell Biol 22(4): 528-534.

Hutagalung, A. H. and P. J. Novick (2011). "Role of Rab GTPases in membrane traffic and cell physiology." Physiol Rev 91(1): 119-149.

Idevall-Hagren, O. and P. De Camilli (2015). "Detection and manipulation of phosphoinositides." Biochim Biophys Acta 1851(6): 736-745.

Ikonomov, O. C., D. Sbrissa, et al. (2009). "PIKfyve-ArPIKfyve-Sac3 core complex: contact sites and their consequence for Sac3 phosphatase activity and endocytic membrane homeostasis." J Biol Chem 284(51): 35794-35806.

Ikonomov, O. C., D. Sbrissa, et al. (2003). "PIKfyve controls fluid phase endocytosis but not recycling/degradation of endocytosed receptors or sorting of procathepsin D by regulating multivesicular body morphogenesis." Mol Biol Cell 14(11): 4581-4591. 
Ikonomov, O. C., D. Sbrissa, et al. (2006). "Localized PtdIns 3,5-P2 synthesis to regulate early endosome dynamics and fusion." Am J Physiol Cell Physiol 291(2): C393-404.

Jean, S., S. Cox, et al. (2012). "Sbf/MTMR13 coordinates PI(3)P and Rab21 regulation in endocytic control of cellular remodeling." Mol Biol Cell 23(14): 2723-2740.

Jean, S. and A. A. Kiger (2012). "Coordination between RAB GTPase and phosphoinositide regulation and functions." Nat Rev Mol Cell Biol 13(7): 463-470.

Johnson, E. E., J. H. Overmeyer, et al. (2006). "Gene silencing reveals a specific function of hVps34 phosphatidylinositol 3-kinase in late versus early endosomes." J Cell Sci 119(Pt 7): 1219-1232.

Jovic, M., M. J. Kean, et al. (2014). "Endosomal sorting of VAMP3 is regulated by PI4K2A." J Cell Sci 127(Pt 17): 3745-3756.

Jungmichel, S., K. B. Sylvestersen, et al. (2014). "Specificity and commonality of the phosphoinositide-binding proteome analyzed by quantitative mass spectrometry." Cell Rep 6(3): 578-591.

Kim, S. A., G. S. Taylor, et al. (2002). "Myotubularin and MTMR2, phosphatidylinositol 3phosphatases mutated in myotubular myopathy and type 4B Charcot-Marie-Tooth disease." J Biol Chem 277(6): 4526-4531.

Kioschis, P., S. Wiemann, et al. (1998). "Genomic organization of a 225-kb region in Xq28 containing the gene for X-linked myotubular myopathy (MTM1) and a related gene (MTMR1)." Genomics 54(2): 256-266.

Kouranti, I., M. Sachse, et al. (2006). "Rab35 regulates an endocytic recycling pathway essential for the terminal steps of cytokinesis." Curr Biol 16(17): 1719-1725.

Krauss, M. and V. Haucke (2007). "Phosphoinositide-metabolizing enzymes at the interface between membrane traffic and cell signalling." EMBO Rep 8(3): 241-246.

Krauss, M. and V. Haucke (2007). "Phosphoinositides: regulators of membrane traffic and protein function." FEBS Lett 581(11): 2105-2111.

Krauss, M., V. Kukhtina, et al. (2006). "Stimulation of phosphatidylinositol kinase type Imediated phosphatidylinositol (4,5)-bisphosphate synthesis by AP-2mu-cargo complexes." Proc Natl Acad Sci U S A 103(32): 11934-11939.

Ktistakis, N. T. and S. A. Tooze (2013). "PIPing on lysosome tubes." EMBO J 32(3): 315317.

Kubo, K., M. Kobayashi, et al. (2015). "SNAP23/25 and VAMP2 mediate exocytic event of transferrin receptor-containing recycling vesicles." Biol Open 4(7): 910-920. 
Laemmli, U. K. (1970). "Cleavage of structural proteins during the assembly of the head of bacteriophage T4." Nature 227(5259): 680-685.

Laporte, J., V. Biancalana, et al. (2000). "MTM1 mutations in X-linked myotubular myopathy." Hum Mutat 15(5): 393-409.

Laporte, J., F. Blondeau, et al. (2002). "The PtdIns3P phosphatase myotubularin is a cytoplasmic protein that also localizes to Rac1-inducible plasma membrane ruffles." J Cell Sci 115(Pt 15): 3105-3117.

Laporte, J., W. Kress, et al. (2001). "Diagnosis of X-linked myotubular myopathy by detection of myotubularin." Ann Neurol 50(1): 42-46.

Laporte, J., L. Liaubet, et al. (2002). "Functional redundancy in the myotubularin family." Biochem Biophys Res Commun 291(2): 305-312.

Lee, H. W., Y. Kim, et al. (2010). "The phosphoinositide 3-phosphatase MTMR2 interacts with PSD-95 and maintains excitatory synapses by modulating endosomal traffic." J Neurosci 30(16): 5508-5518.

Lemmon, M. A. (2008). "Membrane recognition by phospholipid-binding domains." Nat Rev Mol Cell Biol 9(2): 99-111.

Lindmo, K. and H. Stenmark (2006). "Regulation of membrane traffic by phosphoinositide 3kinases." J Cell Sci 119(Pt 4): 605-614.

Lorenzo, O., S. Urbe, et al. (2006). "Systematic analysis of myotubularins: heteromeric interactions, subcellular localisation and endosome related functions." J Cell Sci 119(Pt 14): 2953-2959.

Lu, D., H. Q. Sun, et al. (2012). "Phosphatidylinositol 4-kinase IIalpha is palmitoylated by Golgi-localized palmitoyltransferases in cholesterol-dependent manner." J Biol Chem 287(26): 21856-21865.

Maritzen, T., M. R. Schmidt, et al. (2010). "A novel subtype of AP-1-binding motif within the palmitoylated trans-Golgi network/endosomal accessory protein Gadkin/gamma-BAR." J Biol Chem 285(6): 4074-4086.

McCartney, A. J., Y. Zhang, et al. (2014). "Phosphatidylinositol 3,5-bisphosphate: low abundance, high significance." Bioessays 36(1): 52-64.

Meunier, F. A., S. L. Osborne, et al. (2005). "Phosphatidylinositol 3-kinase C2alpha is essential for ATP-dependent priming of neurosecretory granule exocytosis." Mol Biol Cell 16(10): 4841-4851.

Minogue, S., M. G. Waugh, et al. (2006). "Phosphatidylinositol 4-kinase is required for endosomal trafficking and degradation of the EGF receptor." J Cell Sci 119(Pt 3): 571-581. 
Mizuno-Yamasaki, E., M. Medkova, et al. (2010). "Phosphatidylinositol 4-phosphate controls both membrane recruitment and a regulatory switch of the Rab GEF Sec2p." Dev Cell 18(5): 828-840.

Mizushima, N., B. Levine, et al. (2008). "Autophagy fights disease through cellular selfdigestion." Nature 451(7182): 1069-1075.

Mochizuki, Y. and P. W. Majerus (2003). "Characterization of myotubularin-related protein 7 and its binding partner, myotubularin-related protein 9." Proc Natl Acad Sci U S A 100(17): 9768-9773.

Mossinger, J., M. Wieffer, et al. (2012). "Phosphatidylinositol 4-kinase IIalpha function at endosomes is regulated by the ubiquitin ligase Itch." EMBO Rep 13(12): 1087-1094.

Mruk, D. D. and C. Y. Cheng (2011). "The myotubularin family of lipid phosphatases in disease and in spermatogenesis." Biochem J 433(2): 253-262.

Munson, M. J., G. F. Allen, et al. (2015). "mTOR activates the VPS34-UVRAG complex to regulate autolysosomal tubulation and cell survival." EMBO J 34(17): 2272-2290.

Murray, J. T., C. Panaretou, et al. (2002). "Role of Rab5 in the recruitment of hVps34/p150 to the early endosome." Traffic 3(6): 416-427.

Nakatsu, F., M. Messa, et al. (2015). "Sac2/INPP5F is an inositol 4-phosphatase that functions in the endocytic pathway." J Cell Biol 209(1): 85-95.

Nakatsu, F., R. M. Perera, et al. (2010). "The inositol 5-phosphatase SHIP2 regulates endocytic clathrin-coated pit dynamics." J Cell Biol 190(3): 307-315.

Nandez, R., D. M. Balkin, et al. (2014). "A role of OCRL in clathrin-coated pit dynamics and uncoating revealed by studies of Lowe syndrome cells." Elife 3: e02975.

Nandurkar, H. H., M. Layton, et al. (2003). "Identification of myotubularin as the lipid phosphatase catalytic subunit associated with the 3-phosphatase adapter protein, 3-PAP." Proc Natl Acad Sci U S A 100(15): 8660-8665.

Naughtin, M. J., D. A. Sheffield, et al. (2010). "The myotubularin phosphatase MTMR4 regulates sorting from early endosomes." J Cell Sci 123(Pt 18): 3071-3083.

Omary, M. B., P. A. Coulombe, et al. (2004). "Intermediate filament proteins and their associated diseases." N Engl J Med 351(20): 2087-2100.

Oppelt, A., V. H. Lobert, et al. (2013). "Production of phosphatidylinositol 5-phosphate via PIKfyve and MTMR3 regulates cell migration." EMBO Rep 14(1): 57-64. 
Ortiz, D., M. Medkova, et al. (2002). "Ypt32 recruits the Sec4p guanine nucleotide exchange factor, Sec $2 p$, to secretory vesicles; evidence for a Rab cascade in yeast." J Cell Biol 157(6): 1005-1015.

Parrish, W. R., C. J. Stefan, et al. (2004). "Essential role for the myotubularin-related phosphatase Ymrlp and the synaptojanin-like phosphatases Sj12p and Sj13p in regulation of phosphatidylinositol 3-phosphate in yeast." Mol Biol Cell 15(8): 3567-3579.

Perera, R. M., R. Zoncu, et al. (2006). "Two synaptojanin 1 isoforms are recruited to clathrincoated pits at different stages." Proc Natl Acad Sci U S A 103(51): 19332-19337.

Petiot, A., J. Faure, et al. (2003). "PI3P signaling regulates receptor sorting but not transport in the endosomal pathway." J Cell Biol 162(6): 971-979.

Polevoy, G., H. C. Wei, et al. (2009). "Dual roles for the Drosophila PI 4-kinase four wheel drive in localizing Rab11 during cytokinesis." J Cell Biol 187(6): 847-858.

Posor, Y., M. Eichhorn-Gruenig, et al. (2013). "Spatiotemporal control of endocytosis by phosphatidylinositol-3,4-bisphosphate." Nature 499(7457): 233-237.

Posor, Y., M. Eichhorn-Grunig, et al. (2015). "Phosphoinositides in endocytosis." Biochim Biophys Acta 1851(6): 794-804.

Previtali, S. C., B. Zerega, et al. (2003). "Myotubularin-related 2 protein phosphatase and neurofilament light chain protein, both mutated in CMT neuropathies, interact in peripheral nerve." Hum Mol Genet 12(14): 1713-1723.

Qin, Y., L. Li, et al. (2009). "Regulation of phosphatidylinositol kinases and metabolism by Wnt3a and Dvl." J Biol Chem 284(34): 22544-22548.

Raiborg, C., K. O. Schink, et al. (2013). "Class III phosphatidylinositol 3-kinase and its catalytic product PtdIns3P in regulation of endocytic membrane traffic." FEBS J 280(12): 2730-2742.

Rainero, E. and J. C. Norman (2013). "Late endosomal and lysosomal trafficking during integrin-mediated cell migration and invasion: cell matrix receptors are trafficked through the late endosomal pathway in a way that dictates how cells migrate." Bioessays 35(6): 523-532.

Ravenscroft, G., N. G. Laing, et al. (2015). "Pathophysiological concepts in the congenital myopathies: blurring the boundaries, sharpening the focus." Brain 138(Pt 2): 246-268.

Ribeiro, I., L. Yuan, et al. (2011). "Phosphoinositide regulation of integrin trafficking required for muscle attachment and maintenance." PLoS Genet 7(2): e1001295.

Sanger, F., S. Nicklen, et al. (1977). "DNA sequencing with chain-terminating inhibitors." Proc Natl Acad Sci U S A 74(12): 5463-5467. 
Santiago-Tirado, F. H. and A. Bretscher (2011). "Membrane-trafficking sorting hubs: cooperation between PI4P and small GTPases at the trans-Golgi network." Trends Cell Biol 21(9): 515-525.

Santoro, M., A. Modoni, et al. (2010). "Analysis of MTMR1 expression and correlation with muscle pathological features in juvenile/adult onset myotonic dystrophy type 1 (DM1) and in myotonic dystrophy type 2 (DM2)." Exp Mol Pathol 89(2): 158-168.

Sbrissa, D., O. C. Ikonomov, et al. (2007). "Core protein machinery for mammalian phosphatidylinositol 3,5-bisphosphate synthesis and turnover that regulates the progression of endosomal transport. Novel Sac phosphatase joins the ArPIKfyve-PIKfyve complex." J Biol Chem 282(33): 23878-23891.

Schaletzky, J., S. K. Dove, et al. (2003). "Phosphatidylinositol-5-phosphate activation and conserved substrate specificity of the myotubularin phosphatidylinositol 3-phosphatases." Curr Biol 13(6): 504-509.

Schink, K. O., C. Raiborg, et al. (2013). "Phosphatidylinositol 3-phosphate, a lipid that regulates membrane dynamics, protein sorting and cell signalling." Bioessays 35(10): 900912.

Shen, J., W. M. Yu, et al. (2009). "Deficiency of MIP/MTMR14 phosphatase induces a muscle disorder by disrupting $\mathrm{Ca}(2+)$ homeostasis." Nat Cell Biol 11(6): 769-776.

Shin, H. W., M. Hayashi, et al. (2005). "An enzymatic cascade of Rab5 effectors regulates phosphoinositide turnover in the endocytic pathway." J Cell Biol 170(4): 607-618.

Simons, J. P., R. Al-Shawi, et al. (2009). "Loss of phosphatidylinositol 4-kinase 2alpha activity causes late onset degeneration of spinal cord axons." Proc Natl Acad Sci U S A 106(28): 11535-11539.

Sridhar, S., B. Patel, et al. (2013). "The lipid kinase PI4KIIIbeta preserves lysosomal identity." EMBO J 32(3): 324-339.

Srivastava, S., Z. Li, et al. (2005). "The phosphatidylinositol 3-phosphate phosphatase myotubularin- related protein 6 (MTMR6) is a negative regulator of the $\mathrm{Ca} 2+$-activated $\mathrm{K}+$ channel KCa3.1." Mol Cell Biol 25(9): 3630-3638.

Steinberg, F., K. J. Heesom, et al. (2012). "SNX17 protects integrins from degradation by sorting between lysosomal and recycling pathways." J Cell Biol 197(2): 219-230.

Stuffers, S., L. Malerod, et al. (2010). "Time-resolved ultrastructural detection of phosphatidylinositol 3-phosphate." J Histochem Cytochem 58(11): 1025-1032.

Subramanian, D., V. Laketa, et al. (2010). "Activation of membrane-permeant caged PtdIns(3)P induces endosomal fusion in cells." Nat Chem Biol 6(5): 324-326. 
Sun, Y., N. Thapa, et al. (2013). "Phosphatidylinositol 4,5-bisphosphate: targeted production and signaling." Bioessays 35(6): 513-522.

Takahashi, S., K. Kubo, et al. (2012). "Rab11 regulates exocytosis of recycling vesicles at the plasma membrane." J Cell Sci 125(Pt 17): 4049-4057.

Takatori, S., T. Tatematsu, et al. (2015). "Phosphatidylinositol 3,5-bisphosphate-rich membrane domains in endosomes and lysosomes." Traffic.

Taylor, G. S., T. Maehama, et al. (2000). "Myotubularin, a protein tyrosine phosphatase mutated in myotubular myopathy, dephosphorylates the lipid second messenger, phosphatidylinositol 3-phosphate." Proc Natl Acad Sci U S A 97(16): 8910-8915.

Temkin, P., B. Lauffer, et al. (2011). "SNX27 mediates retromer tubule entry and endosometo-plasma membrane trafficking of signalling receptors." Nat Cell Biol 13(6): 715-721.

Thapa, N., Y. Sun, et al. (2012). "Phosphoinositide signaling regulates the exocyst complex and polarized integrin trafficking in directionally migrating cells." Dev Cell 22(1): 116-130.

Traer, C. J., A. C. Rutherford, et al. (2007). "SNX4 coordinates endosomal sorting of TfnR with dynein-mediated transport into the endocytic recycling compartment." Nat Cell Biol 9(12): 1370-1380.

Tsujita, K., T. Itoh, et al. (2004). "Myotubularin regulates the function of the late endosome through the gram domain-phosphatidylinositol 3,5-bisphosphate interaction." J Biol Chem 279(14): 13817-13824.

Vallis, Y., P. Wigge, et al. (1999). "Importance of the pleckstrin homology domain of dynamin in clathrin-mediated endocytosis." Curr Biol 9(5): 257-260.

van Weering, J. R., P. Verkade, et al. (2010). "SNX-BAR proteins in phosphoinositidemediated, tubular-based endosomal sorting." Semin Cell Dev Biol 21(4): 371-380.

van Weering, J. R., P. Verkade, et al. (2012). "SNX-BAR-mediated endosome tubulation is co-ordinated with endosome maturation." Traffic 13(1): 94-107.

Velichkova, M., J. Juan, et al. (2010). "Drosophila Mtm and class II PI3K coregulate a PI(3)P pool with cortical and endolysosomal functions." J Cell Biol 190(3): 407-425.

Verstreken, P., T. W. Koh, et al. (2003). "Synaptojanin is recruited by endophilin to promote synaptic vesicle uncoating." Neuron 40(4): 733-748.

Vicinanza, M., V. I. Korolchuk, et al. (2015). "PI(5)P regulates autophagosome biogenesis." Mol Cell 57(2): 219-234. 
Wang, J., H. Q. Sun, et al. (2007). "PI4P promotes the recruitment of the GGA adaptor proteins to the trans-Golgi network and regulates their recognition of the ubiquitin sorting signal." Mol Biol Cell 18(7): 2646-2655.

Wang, Y. J., J. Wang, et al. (2003). "Phosphatidylinositol 4 phosphate regulates targeting of clathrin adaptor AP-1 complexes to the Golgi." Cell 114(3): 299-310.

Wen, P. J., S. L. Osborne, et al. (2008). "Ca2+-regulated pool of phosphatidylinositol-3phosphate produced by phosphatidylinositol 3-kinase C2alpha on neurosecretory vesicles." Mol Biol Cell 19(12): 5593-5603.

Wenk, M. R., L. Lucast, et al. (2003). "Phosphoinositide profiling in complex lipid mixtures using electrospray ionization mass spectrometry." Nat Biotechnol 21(7): 813-817.

Wheeler, M. and J. Domin (2006). "The N-terminus of phosphoinositide 3-kinase-C2beta regulates lipid kinase activity and binding to clathrin." J Cell Physiol 206(3): 586-593.

Wieffer, M., E. Cibrian Uhalte, et al. (2013). "PI4K2beta/AP-1-based TGN-endosomal sorting regulates Wnt signaling." Curr Biol 23(21): 2185-2190.

Wu, B. and W. Guo (2015). "The Exocyst at a Glance." J Cell Sci 128(16): 2957-2964.

Wu, Y., S. Cheng, et al. (2014). "PI3P phosphatase activity is required for autophagosome maturation and autolysosome formation." EMBO Rep 15(9): 973-981.

Xhabija, B., G. S. Taylor, et al. (2011). "Receptor mediated endocytosis 8 is a novel PI(3)P binding protein regulated by myotubularin-related 2." FEBS Lett 585(12): 1722-1728.

Xiong, X., Q. Xu, et al. (2012). "An association between type Igamma PI4P 5-kinase and Exo70 directs E-cadherin clustering and epithelial polarization." Mol Biol Cell 23(1): 87-98.

Yu, J., X. He, et al. (2013). "Myotubularin-related protein 4 (MTMR4) attenuates BMP/Dpp signaling by dephosphorylation of Smad proteins." J Biol Chem 288(1): 79-88.

Yu, J., L. Pan, et al. (2010). "MTMR4 attenuates transforming growth factor beta (TGFbeta) signaling by dephosphorylating R-Smads in endosomes." J Biol Chem 285(11): 8454-8462.

Zhang, X. M., S. Ellis, et al. (2004). "Sec15 is an effector for the Rab11 GTPase in mammalian cells." J Biol Chem 279(41): 43027-43034.

Zoncu, R., R. M. Perera, et al. (2009). "A phosphoinositide switch controls the maturation and signaling properties of APPL endosomes." Cell 136(6): 1110-1121.

Zoncu, R., R. M. Perera, et al. (2007). "Loss of endocytic clathrin-coated pits upon acute depletion of phosphatidylinositol 4,5-bisphosphate." Proc Natl Acad Sci U S A 104(10): 3793-3798. 


\section{Appendix}

\subsection{Appendix A: Abbreviations}

aa

amino acid

ALR autophagosome-lysosome reformation

AP adaptor protein complex

Atg Autophagy-related

BafA1 Bafilomycin A1

BAR Bin1 / amphiphysin / Rvs

BSA bovine serum albumin

C1 HeLaM Clone 1

CC coiled-coil

$\mathrm{CCV} \quad$ clathrin coated vesicle

CCP clathrin coated pit

cds coding sequence

CLIC3 chloride intracellular channel protein 3

CME clathrin mediated endocytosis

CMT Charcot-Marie-Tooth disease

ctrl control

Ctx Cholera toxin

DMSO dimethyl sulfoxide

DRM desmin-related myopathy

DSP dual specific phosphatase

EE early endosome

EEA1 early endosomal antigen 1

EGF epidermal growth factor

EGFR epidermal growth factor receptor

ER endoplasmic reticulum

ERC endocytic recycling compartment

FL full length

FYVE Fab1, YOTB, Vac1, EEA1

$\mathrm{Fz}$

Frizzled 
eGFP/GFP (enhanced) green fluorescent protein

GSDB Goat serum dilution buffer

GST Glutathione S-transferase

GRAM glycosyltransferase, Rab-like GTPase activator and myotubularin

HA hemagglutinin

HDFa human dermal fibroblast from adult healthy individual

HRP horseradish peroxidase

IF intermediate filament

IP immunoprecipitation

$\mathrm{I}(1,3) \mathrm{P}_{2} \quad$ inositol-1,3-bisphosphate

KD knockdown

KO knockout

LC3 microtubule-associated protein 1 light chain 3

MS/MS tandem mass spectrometry

mRNA messenger RNA

MTM myotubularin

MTMR myotubularin related

MVB multi vesicular body

OCRL oculocerebrorenal syndrome of Lowe

PBS phosphate buffered saline

PCR Polymerase chain reaction

PD pull down

PDZ post synaptic density

PDZB PDZ binding motif

PFA paraformaldehyde

PH pleckstrin-homology

Phox phagocytic oxidase

PI phosphoinositide

PI(3)P phosphatidylinositol-3-phosphate

$\mathrm{PI}(3,4) \mathrm{P}_{2} \quad$ phosphatidylinositol-3,4-bisphosphate

$\mathrm{PI}(3,4,5) \mathrm{P}_{3}$ phosphatidylinositol-3,4,5-trissphosphate

PI(4)P phosphatidylinositol-4-phosphate

$\mathrm{PI}(4,5) \mathrm{P}_{2} \quad$ phosphatidylinositol-4,5-bisphosphate

PI3K phosphatidylinositol 3-kinase 
PI4K phosphatidylinositol 4-kinase

PIKfyve phosphoinositide kinase, FYVE finger containing

PIP phosphatidylinositol phosphate

PIP4K phosphatidylinositol-5-phosphate 4-kinase

PIP5K phosphatidylinositol-4-phosphate 5-kinase

PLC phospholipase C

PS phosphatidylserine

PTP protein tyrosine phosphatase

PX Phox homology

RID Rac-induced recruitment domain

RE recycling endosome

RyR ryanodine receptor

scr scrambled

SDS-PAGE Sodiumdodecylsulfate polyacrylamide gel electrophoresis

SE sorting endosome

SID SET-interacting domain

siRNA small interfering RNA

SNARE soluble N-ethylmaleimide-sensitive-factor attachment receptor

t-SNARE target - SNARE

v-SNARE vesicular-SNARE

SNX sorting nexin

SR sarcoplasmic reticulum

TBS Tris-buffered saline

Tf Transferrin

TfR Transferrin receptor

TGN trans-Golgi-network

TIRF Total internal reflection microscopy

tv transcription variant

VAMP vesicle associated membrane protein

Vps vacuolar protein sorting

VSV-G vesicular stomatits virus G protein

WM Wortmannin

wt wild-type

XLCNM X-linked centronuclear myopathy 


\subsection{Appendix B: Mass spectrometry results}

\subsubsection{MTM1 binding partners identified by mass spectrometry}

\section{Table 10: MTM1 binding partner identified by MS/MS-based mass spectrometry}

Only MS/MS-results with enrichment in the heavy metal labelled fraction are listed here. The threshold was arbitrarily set to $\mathrm{H} / \mathrm{L}>1.8$. Experiments were performed in collaboration with Eberhard Krause. H/L - heavy/ light ratio; \# - number of unique peptides

\begin{tabular}{|c|c|c|}
\hline H/L & \# & Description \\
\hline $6,77 * 10^{6}$ & 2 & Developmentally-regulated GTP-binding protein $1 \mathrm{OS}=$ Homo sapiens GN=DRG1 PE=1 SV=1 \\
\hline $1,07 * 10^{6}$ & 2 & Probable ATP-dependent RNA helicase DDX27 OS=Homo sapiens GN=DDX27 PE=1 SV=2 \\
\hline $1,03 * 10^{6}$ & 2 & Midasin OS=Homo sapiens GN=MDN1 PE=1 SV=2 \\
\hline $5,78 * 10^{5}$ & 5 & E3 ubiquitin-protein ligase TRIM17 (Fragment) OS=Homo sapiens GN=TRIM17 PE=4 SV=1 \\
\hline $2,32 * 10^{5}$ & 2 & Guanine nucleotide-binding protein-like 3-like protein OS=Homo sapiens GN=GNL3L PE=1 SV=1 \\
\hline $1,34 * 10^{5}$ & 23 & U5 small nuclear ribonucleoprotein $200 \mathrm{kDa}$ helicase OS=Homo sapiens GN=SNRNP200 PE=1 SV=2 \\
\hline $1,25 * 10^{5}$ & 2 & Guanine nucleotide-binding protein-like $3 \mathrm{OS}=$ Homo sapiens $\mathrm{GN}=\mathrm{GNL} 3 \mathrm{PE}=1 \mathrm{SV}=2$ \\
\hline $1,24 * 10^{5}$ & 2 & Replication factor C subunit $4 \mathrm{OS}=$ Homo sapiens $\mathrm{GN}=\mathrm{RFC} 4 \mathrm{PE}=2 \mathrm{SV}=1$ \\
\hline $6,41 * 10^{4}$ & 4 & DnaJ homolog subfamily $\mathrm{C}$ member $21 \mathrm{OS}=$ Homo sapiens $\mathrm{GN}=$ DNAJC21 $\mathrm{PE}=1 \mathrm{SV}=2$ \\
\hline $2,76 * 10^{4}$ & 5 & T-complex protein 1 subunit gamma OS=Homo sapiens GN=CCT3 PE=1 SV=4 \\
\hline $2,06 * 10^{4}$ & 2 & Uncharacterized protein KIAA1671 OS=Homo sapiens GN=KIAA1671 PE=1 SV=2 \\
\hline $1,75^{*} 10^{4}$ & 4 & Eukaryotic translation initiation factor 4 gamma $2 \mathrm{OS}=$ Homo sapiens GN=EIF4G2 PE=2 SV=1 \\
\hline 9071 & 2 & Zinc finger protein $593 \mathrm{OS}=$ Homo sapiens $\mathrm{GN}=\mathrm{ZNF} 593 \mathrm{PE}=1 \mathrm{SV}=2$ \\
\hline 5259 & 3 & Whirlin OS=Homo sapiens GN=DFNB31 PE $=1 \mathrm{SV}=3$ \\
\hline 5061 & 2 & DNA-binding protein SMUBP-2 OS=Homo sapiens $\mathrm{GN}=\mathrm{IGHMBP} 2 \mathrm{PE}=1 \mathrm{SV}=3$ \\
\hline 4455 & 3 & Zinc finger CCCH-type antiviral protein $1 \mathrm{OS}=$ Homo sapiens GN=ZC3HAV1 PE=1 SV=3 \\
\hline 4012 & 2 & Ras association domain-containing protein 8 (Fragment) OS=Homo sapiens GN=RASSF8 PE=2 SV=1 \\
\hline 3105 & 6 & Gamma-tubulin complex component 3 OS=Homo sapiens GN=TUBGCP3 PE=1 SV=2 \\
\hline 2173 & 5 & ATP-binding cassette sub-family F member $1 \mathrm{OS}=$ Homo sapiens $\mathrm{GN}=\mathrm{ABCF} 1 \mathrm{PE}=1 \mathrm{SV}=2$ \\
\hline 1724 & 2 & $26 \mathrm{~S}$ protease regulatory subunit $7 \mathrm{OS}=$ Homo sapiens $\mathrm{GN}=\mathrm{PSMC} 2 \mathrm{PE}=2 \mathrm{SV}=1$ \\
\hline 1564 & 4 & Replication factor $\mathrm{C}$ subunit $2 \mathrm{OS}=$ Homo sapiens $\mathrm{GN}=\mathrm{RFC} 2 \mathrm{PE}=1 \mathrm{SV}=3$ \\
\hline 900,2 & 5 & 60S ribosomal export protein NMD3 OS=Homo sapiens GN=NMD3 PE=2 SV=1 \\
\hline 745,3 & 12 & Eukaryotic translation initiation factor 3 subunit D OS=Homo sapiens GN=EIF3D PE=1 SV=1 \\
\hline 718 & 2 & Histone-lysine N-methyltransferase SETD1A OS=Homo sapiens GN=SETD1A PE=1 SV=3 \\
\hline 570,6 & 3 & Isoform 10 of Titin OS=Homo sapiens GN=TTN \\
\hline $\mathbf{5 2 5 , 7}$ & 3 & Myotubularin-related protein $10 \mathrm{OS}=$ Homo sapiens GN=MTMR10 PE=1 SV=3 \\
\hline 490,8 & 4 & 60S ribosomal protein $\mathrm{L} 26 \mathrm{OS}=$ Homo sapiens GN=RPL26 PE=1 SV=1 \\
\hline 462,2 & 2 & Importin subunit alpha-1 OS=Homo sapiens GN=KPNA2 PE=1 SV=1 \\
\hline 455,3 & 2 & Olfactory receptor $1 \mathrm{~L} 3 \mathrm{OS}=$ Homo sapiens GN=OR1L3 PE=2 SV=1 \\
\hline 394 & 12 & Heterogeneous nuclear ribonucleoprotein K OS=Homo sapiens GN=HNRNPK PE=1 SV=1 \\
\hline 358,4 & 2 & Transducin beta-like protein $3 \mathrm{OS}=$ Homo sapiens $\mathrm{GN}=\mathrm{TBL} 3 \mathrm{PE}=1 \mathrm{SV}=2$ \\
\hline 330,7 & 8 & $60 \mathrm{~S}$ ribosomal protein $\mathrm{L} 27 \mathrm{OS}=$ Homo sapiens $\mathrm{GN}=\mathrm{RPL} 27 \mathrm{PE}=1 \mathrm{SV}=2$ \\
\hline 315,4 & 9 & $60 \mathrm{~S}$ ribosomal protein $\mathrm{L} 21 \mathrm{OS}=$ Homo sapiens $\mathrm{GN}=\mathrm{RPL} 21 \mathrm{PE}=1 \mathrm{SV}=2$ \\
\hline 308,1 & 2 & Glutaredoxin-3 OS=Homo sapiens GN=GLRX3 PE=1 SV=2 \\
\hline 305,8 & 2 & Polypyrimidine tract-binding protein $1 \mathrm{OS}=$ Homo sapiens $\mathrm{GN}=\mathrm{PTBP} 1 \mathrm{PE}=1 \mathrm{SV}=1$ \\
\hline 294,2 & 3 & Phenylalanine--tRNA ligase beta subunit $\mathrm{OS}=$ Homo sapiens $\mathrm{GN}=\mathrm{FARSB} \mathrm{PE}=1 \mathrm{SV}=3$ \\
\hline 265,5 & 3 & ATP-dependent RNA helicase DDX1 OS=Homo sapiens GN=DDX1 PE=1 SV=2 \\
\hline 263,4 & 39 & Junction plakoglobin $\mathrm{OS}=$ Homo sapiens $\mathrm{GN}=\mathrm{JUP} \mathrm{PE}=1 \mathrm{SV}=3$ \\
\hline 249,6 & 4 & $60 \mathrm{~S}$ ribosomal protein $\mathrm{L} 15 \mathrm{OS}=\mathrm{Homo}$ sapiens $\mathrm{GN}=\mathrm{RPL} 15 \mathrm{PE}=1 \mathrm{SV}=2$ \\
\hline 242,5 & 3 & Nucleolar RNA helicase 2 OS=Homo sapiens GN=DDX21 PE=1 SV=5 \\
\hline 228,7 & 3 & WD repeat-containing protein mio OS=Homo sapiens GN=MIOS PE $=1 \mathrm{SV}=2$ \\
\hline 222,3 & 69 & Epiplakin OS=Homo sapiens GN=EPPK1 PE=1 SV=2 \\
\hline 221,7 & 12 & Cation channel sperm-associated protein subunit gamma OS=Homo sapiens $\mathrm{GN}=\mathrm{CATSPERG} \mathrm{PE}=2 \mathrm{SV}=3$ \\
\hline 207,6 & 5 & EBNA1 binding protein 2, isoform CRA_d OS=Homo sapiens GN=EBNA1BP2 PE=4 SV=1 \\
\hline 200,1 & 21 & 40S ribosomal protein $\mathrm{S} 18 \mathrm{OS}=$ Homo sapiens $\mathrm{GN}=\mathrm{RPS} 18 \mathrm{PE}=1 \mathrm{SV}=3$ \\
\hline 184,5 & 3 & Exosome complex component RRP41 OS=Homo sapiens GN=EXOSC4 PE=1 SV=3 \\
\hline 183,8 & 3 & Zinc finger protein $622 \mathrm{OS}=$ Homo sapiens $\mathrm{GN}=\mathrm{ZNF} 622 \mathrm{PE}=1 \mathrm{SV}=1$ \\
\hline 181,7 & 8 & 40S ribosomal protein $\mathrm{S} 16 \mathrm{OS}=\mathrm{Homo}$ sapiens $\mathrm{GN}=\mathrm{RPS} 16 \mathrm{PE}=1 \mathrm{SV}=2$ \\
\hline 180,4 & 17 & Eukaryotic translation initiation factor 3 subunit L OS=Homo sapiens GN=EIF3L PE=2 SV=1 \\
\hline 180,3 & 11 & TRMT1-like protein OS=Homo sapiens GN=TRMT1L PE=1 SV=2 \\
\hline 177 & 10 & tRNA (cytosine(34)-C(5))-methyltransferase OS=Homo sapiens GN=NSUN2 PE=1 SV=2 \\
\hline 175,1 & 4 & Tubulin gamma- 1 chain $\mathrm{OS}=$ Homo sapiens $\mathrm{GN}=\mathrm{TUBG} 1 \mathrm{PE}=1 \mathrm{SV}=2$ \\
\hline 170,1 & 3 & Structural maintenance of chromosomes protein $1 \mathrm{~A} \mathrm{OS}=$ Homo sapiens $\mathrm{GN}=\mathrm{SMC} 1 \mathrm{~A} \mathrm{PE}=1 \mathrm{SV}=2$ \\
\hline
\end{tabular}




\begin{tabular}{|c|c|c|}
\hline $\mathrm{H} / \mathrm{L}$ & \# & Description \\
\hline 149,2 & 6 & $60 \mathrm{~S}$ ribosomal protein L17 (Fragment) $\mathrm{OS}=$ Homo sapiens $\mathrm{GN}=\mathrm{RPL} 17 \mathrm{PE}=3 \mathrm{SV}=1$ \\
\hline 147,4 & 2 & 60S ribosomal protein L26-like $1 \mathrm{OS}=$ Homo sapiens GN=RPL26L1 PE=1 SV=1 \\
\hline 145,8 & 7 & Cell growth-regulating nucleolar protein $\mathrm{OS}=$ Homo sapiens $\mathrm{GN}=\mathrm{LYAR} \mathrm{PE}=1 \mathrm{SV}=2$ \\
\hline 143,1 & 123 & Eukaryotic translation initiation factor 3 subunit A OS=Homo sapiens GN=EIF3A PE=1 SV=1 \\
\hline 141,1 & 11 & 40S ribosomal protein $\mathrm{S} 14 \mathrm{OS}=$ Homo sapiens $\mathrm{GN}=\mathrm{RPS} 14 \mathrm{PE}=1 \mathrm{SV}=3$ \\
\hline 140,4 & 25 & Eukaryotic translation initiation factor 3 subunit I OS=Homo sapiens GN=EIF3I PE=1 SV=1 \\
\hline 133,3 & 12 & Eukaryotic translation initiation factor 2 subunit $3 \mathrm{OS}=$ Homo sapiens $\mathrm{GN}=\mathrm{EIF} 2 \mathrm{~S} 3 \mathrm{PE}=1 \mathrm{SV}=3$ \\
\hline 131,3 & 6 & 60S ribosomal protein $\mathrm{L} 24 \mathrm{OS}=\mathrm{Homo}$ sapiens $\mathrm{GN}=\mathrm{RPL} 24 \mathrm{PE}=4 \mathrm{SV}=1$ \\
\hline 130,9 & 31 & Isoform 3 of Plasminogen activator inhibitor 1 RNA-binding protein OS=Homo sapiens GN=SERBP1 \\
\hline 129,6 & 8 & 40S ribosomal protein $\mathrm{S} 13 \mathrm{OS}=$ Homo sapiens $\mathrm{GN}=\mathrm{RPS} 13 \mathrm{PE}=2 \mathrm{SV}=1$ \\
\hline 126,6 & 4 & Bystin OS=Homo sapiens GN=BYSL PE $=1 \mathrm{SV}=3$ \\
\hline 123,9 & 381 & Myotubularin OS=Homo sapiens GN=MTM1 PE=1 SV=2 \\
\hline 121 & 14 & Exosome component $10 \mathrm{OS}=$ Homo sapiens $\mathrm{GN}=\mathrm{EXOSC} 10 \mathrm{PE}=1 \mathrm{SV}=2$ \\
\hline 120,9 & 6 & Double-stranded RNA-binding protein Staufen homolog $1 \mathrm{OS}=$ Homo sapiens $\mathrm{GN}=\mathrm{STAU} 1 \mathrm{PE}=2 \mathrm{SV}=1$ \\
\hline 116,9 & 51 & Eukaryotic translation initiation factor 3 subunit $\mathrm{C} \mathrm{OS}=$ Homo sapiens $\mathrm{GN}=\mathrm{EIF} 3 \mathrm{C} \mathrm{PE}=2 \mathrm{SV}=1$ \\
\hline 116,6 & 5 & 60S ribosomal protein $\mathrm{L} 31 \mathrm{OS}=$ Homo sapiens $\mathrm{GN}=\mathrm{RPL} 31 \mathrm{PE}=2 \mathrm{SV}=1$ \\
\hline 115,6 & 2 & DNA-directed RNA polymerases I and III subunit RPAC1 OS=Homo sapiens GN=POLR1C PE=1 SV=1 \\
\hline 114 & 6 & 40S ribosomal protein $\mathrm{S} 23 \mathrm{OS}=$ Homo sapiens $\mathrm{GN}=\mathrm{RPS} 23 \mathrm{PE}=1 \mathrm{SV}=3$ \\
\hline 110,9 & 7 & $60 \mathrm{~S}$ ribosomal protein $\mathrm{L} 18 \mathrm{a} \mathrm{OS}=$ Homo sapiens $\mathrm{GN}=\mathrm{RPL} 18 \mathrm{~A} \mathrm{PE}=3 \mathrm{SV}=1$ \\
\hline 110,2 & 3 & $60 \mathrm{~S}$ ribosomal protein $\mathrm{L} 36 \mathrm{OS}=\mathrm{Homo}$ sapiens $\mathrm{GN}=\mathrm{RPL} 36 \mathrm{PE}=1 \mathrm{SV}=3$ \\
\hline 108,4 & 3 & p21-activated protein kinase-interacting protein $1 \mathrm{OS}=$ Homo sapiens $\mathrm{GN}=\mathrm{PAK} 1 \mathrm{IP} 1 \mathrm{PE}=1 \mathrm{SV}=2$ \\
\hline 108,3 & 36 & Eukaryotic translation initiation factor 3 subunit E OS=Homo sapiens GN=EIF3E PE=1 SV=1 \\
\hline 104 & 2 & RNA-binding protein NOB1 OS=Homo sapiens GN=NOB1 PE=1 SV=1 \\
\hline 103,4 & 5 & Mov10, Moloney leukemia virus 10 , homolog (Mouse), isoform CRA_a OS=Homo sapiens GN=MOV10 PE=4 SV=1 \\
\hline 103 & 16 & 3-ketoacyl-CoA thiolase OS=Homo sapiens GN=HADHB PE=2 SV=1 \\
\hline 99,94 & 2 & 40S ribosomal protein $\mathrm{S} 26 \mathrm{OS}=\mathrm{Homo}$ sapiens $\mathrm{GN}=\mathrm{RPS} 26 \mathrm{PE}=1 \mathrm{SV}=3$ \\
\hline 97,27 & 10 & 40S ribosomal protein $\mathrm{S} 25 \mathrm{OS}=$ Homo sapiens $\mathrm{GN}=\mathrm{RPS} 25 \mathrm{PE}=1 \mathrm{SV}=1$ \\
\hline 96,67 & 4 & 40S ribosomal protein $\mathrm{S} 11 \mathrm{OS}=$ Homo sapiens $\mathrm{GN}=\mathrm{RPS} 11 \mathrm{PE}=2 \mathrm{SV}=1$ \\
\hline 94,07 & 8 & 40S ribosomal protein $\mathrm{S} 24 \mathrm{OS}=$ Homo sapiens $\mathrm{GN}=\mathrm{RPS} 24 \mathrm{PE}=2 \mathrm{SV}=1$ \\
\hline 92,65 & 2 & Cyclin-dependent kinase 5 activator $2 \mathrm{OS}=$ Homo sapiens $\mathrm{GN}=\mathrm{CDK} 5 \mathrm{R} 2 \mathrm{PE}=1 \mathrm{SV}=1$ \\
\hline 92,1 & 20 & Eukaryotic translation initiation factor 3 subunit $\mathrm{H} \mathrm{OS}=$ Homo sapiens GN=EIF3S3 PE=2 SV=1 \\
\hline 90,9 & 5 & General transcription factor II-I OS=Homo sapiens GN=GTF2I PE=1 SV=2 \\
\hline 83,86 & 2 & M-phase phosphoprotein $6 \mathrm{OS}=$ Homo sapiens $\mathrm{GN}=\mathrm{MPHOSPH} 6 \mathrm{PE}=2 \mathrm{SV}=1$ \\
\hline 82,36 & 4 & WD repeat-containing protein $36 \mathrm{OS}=$ Homo sapiens $\mathrm{GN}=\mathrm{WDR} 36 \mathrm{PE}=1 \mathrm{SV}=1$ \\
\hline 81,53 & 6 & FACT complex subunit SPT16 OS=Homo sapiens GN=SUPT16H PE=1 SV=1 \\
\hline 79,97 & 5 & $60 \mathrm{~S}$ ribosomal protein $\mathrm{L} 23 \mathrm{a} \mathrm{OS}=$ Homo sapiens $\mathrm{GN}=\mathrm{RPL} 23 \mathrm{~A} \mathrm{PE}=1 \mathrm{SV}=1$ \\
\hline 79,6 & 13 & Myotubularin-related protein 1 (Fragment) OS=Homo sapiens GN=MTMR1 PE=4 SV=1 \\
\hline 78,5 & 10 & 40S ribosomal protein $\mathrm{S} 7 \mathrm{OS}=$ Homo sapiens $\mathrm{GN}=\mathrm{RPS} 7 \mathrm{PE}=1 \mathrm{SV}=1$ \\
\hline 77,08 & 7 & $60 \mathrm{~S}$ ribosomal protein $\mathrm{L} 23 \mathrm{OS}=$ Homo sapiens $\mathrm{GN}=\mathrm{RPL} 23 \mathrm{PE}=1 \mathrm{SV}=1$ \\
\hline 76,83 & 12 & Myotubularin-related protein $12 \mathrm{OS}=$ Homo sapiens GN=MTMR12 PE=1 SV=2 \\
\hline 74,5 & 4 & T-complex protein 1 subunit alpha $\mathrm{OS}=$ Homo sapiens $\mathrm{GN}=\mathrm{TCP} 1 \mathrm{PE}=1 \mathrm{SV}=1$ \\
\hline 74,18 & 12 & Staphylococcal nuclease domain-containing protein $1 \mathrm{OS}=$ Homo sapiens $\mathrm{GN}=\mathrm{SND} 1 \mathrm{PE}=1 \mathrm{SV}=1$ \\
\hline 72,52 & 14 & Interleukin enhancer-binding factor $2 \mathrm{OS}=\mathrm{Homo}$ sapiens GN=ILF2 PE=1 SV=2 \\
\hline 72,35 & 8 & 40S ribosomal protein $\mathrm{S} 15 \mathrm{a} \mathrm{OS}=$ Homo sapiens $\mathrm{GN}=\mathrm{RPS} 15 \mathrm{~A} \mathrm{PE}=1 \mathrm{SV}=2$ \\
\hline 71,4 & 6 & Acidic fibroblast growth factor intracellular-binding protein OS=Homo sapiens GN=FIBP PE $=1 \mathrm{SV}=3$ \\
\hline 71,26 & 18 & 40S ribosomal protein $\mathrm{S} 10 \mathrm{OS}=$ Homo sapiens $\mathrm{GN}=\mathrm{RPS} 10 \mathrm{PE}=1 \mathrm{SV}=1$ \\
\hline 71,08 & 30 & Nucleolin OS=Homo sapiens GN=NCL PE=1 SV=3 \\
\hline 69,08 & 5 & Activating signal cointegrator 1 complex subunit $2 \mathrm{OS}=$ Homo sapiens $\mathrm{GN}=\mathrm{ASCC} 2 \mathrm{PE}=1 \mathrm{SV}=3$ \\
\hline 67,61 & 12 & 60S ribosomal protein L9 (Fragment) OS=Homo sapiens GN=RPL9 PE=4 SV=1 \\
\hline 65,37 & 18 & 40S ribosomal protein $\mathrm{S} 15 \mathrm{OS}=$ Homo sapiens $\mathrm{GN}=\mathrm{RPS} 15 \mathrm{PE}=3 \mathrm{SV}=1$ \\
\hline 64,47 & 3 & Vigilin OS=Homo sapiens GN=HDLBP PE=1 SV=2 \\
\hline 64,38 & 6 & Eukaryotic translation initiation factor 3 subunit G OS=Homo sapiens GN=EIF3G PE=1 SV=2 \\
\hline 64,36 & 3 & $60 \mathrm{~S}$ ribosomal protein L22-like 1 (Fragment) $\mathrm{OS}=$ Homo sapiens $\mathrm{GN}=\mathrm{RPL} 22 \mathrm{~L} 1 \mathrm{PE}=4 \mathrm{SV}=1$ \\
\hline 64,24 & 41 & Eukaryotic translation initiation factor 3 subunit $\mathrm{B}$ OS=Homo sapiens GN=EIF3B PE=1 SV=3 \\
\hline 63,39 & 12 & Pre-mRNA-processing-splicing factor $8 \mathrm{OS}=$ Homo sapiens GN=PRPF8 PE=1 SV=2 \\
\hline 63,12 & 23 & $60 \mathrm{~S}$ ribosomal protein $\mathrm{L3} \mathrm{OS}=$ Homo sapiens $\mathrm{GN}=\mathrm{RPL} 3 \mathrm{PE}=1 \mathrm{SV}=2$ \\
\hline 62,17 & 25 & Eukaryotic initiation factor 4A-I OS=Homo sapiens $\mathrm{GN}=\mathrm{EIF} 4 \mathrm{~A} 1 \mathrm{PE}=1 \mathrm{SV}=1$ \\
\hline 61,97 & 2 & Neuropathy target esterase OS=Homo sapiens GN=PNPLA6 PE=1 SV=2 \\
\hline 61,89 & 13 & 40S ribosomal protein $\mathrm{S} 5$ (Fragment) $\mathrm{OS}=$ Homo sapiens $\mathrm{GN}=\mathrm{RPS} 5 \mathrm{PE}=2 \mathrm{SV}=1$ \\
\hline 61,47 & 6 & ATP-dependent RNA helicase DHX29 OS=Homo sapiens GN=DHX29 PE=1 SV=2 \\
\hline 61,3 & 16 & 40S ribosomal protein $\mathrm{S} 19 \mathrm{OS}=$ Homo sapiens $\mathrm{GN}=\mathrm{RPS} 19 \mathrm{PE}=1 \mathrm{SV}=2$ \\
\hline 60,49 & 11 & Trifunctional enzyme subunit alpha, mitochondrial OS=Homo sapiens GN=HADHA PE $=1 \mathrm{SV}=2$ \\
\hline 59,92 & 4 & SRSF protein kinase $1 \mathrm{OS}=$ Homo sapiens $\mathrm{GN}=\mathrm{SRPK} 1 \mathrm{PE}=1 \mathrm{SV}=2$ \\
\hline 58,2 & 37 & Nucleophosmin OS=Homo sapiens GN=NPM1 PE $=1 \mathrm{SV}=2$ \\
\hline 57,33 & 2 & RNA-binding protein $28 \mathrm{OS}=$ Homo sapiens $\mathrm{GN}=\mathrm{RBM} 28 \mathrm{PE}=1 \mathrm{SV}=3$ \\
\hline 56,95 & 12 & 40S ribosomal protein $\mathrm{S} 17$-like $\mathrm{OS}=$ Homo sapiens $\mathrm{GN}=\mathrm{RPS} 17 \mathrm{~L}$ PE $=1 \mathrm{SV}=1$ \\
\hline 56,85 & 5 & Eukaryotic translation initiation factor 3 subunit $\mathrm{K} \mathrm{OS}=$ Homo sapiens $\mathrm{GN}=\mathrm{EIF} 3 \mathrm{~K} \mathrm{PE}=2 \mathrm{SV}=1$ \\
\hline
\end{tabular}




\begin{tabular}{|c|c|c|}
\hline $\mathbf{H} / \mathbf{L}$ & \# & Description \\
\hline 56,77 & 6 & 40S ribosomal protein $\mathrm{S} 20 \mathrm{OS}=$ Homo sapiens $\mathrm{GN}=\mathrm{RPS} 20 \mathrm{PE}=1 \mathrm{SV}=1$ \\
\hline 56,33 & 3 & Cell division cycle 5-like protein $\mathrm{OS}=$ Homo sapiens $\mathrm{GN}=\mathrm{CDC} 5 \mathrm{~L}$ PE $=1 \mathrm{SV}=2$ \\
\hline 55,63 & 27 & Isoform 4 of Interleukin enhancer-binding factor 3 OS=Homo sapiens GN=ILF3 \\
\hline 53,8 & 11 & 60S ribosomal protein $\mathrm{L} 12 \mathrm{OS}=$ Homo sapiens $\mathrm{GN}=\mathrm{RPL} 12 \mathrm{PE}=1 \mathrm{SV}=1$ \\
\hline 53,39 & 7 & Programmed cell death protein $4 \mathrm{OS}=$ Homo sapiens $\mathrm{GN}=\mathrm{PDCD} 4 \mathrm{PE}=1 \mathrm{SV}=2$ \\
\hline 53,13 & 22 & ATP-dependent DNA helicase Q1 OS=Homo sapiens GN=RECQL PE=1 SV=3 \\
\hline 52,73 & 5 & Putative ATP-dependent RNA helicase DHX57 OS=Homo sapiens GN=DHX57 PE=1 SV=2 \\
\hline 51,98 & 4 & $\mathrm{Na}(+) / \mathrm{H}(+)$ exchange regulatory cofactor NHE-RF2 OS=Homo sapiens GN=SLC9A3R2 PE=1 SV=2 \\
\hline 51,73 & 2 & Probable dimethyladenosine transferase OS=Homo sapiens GN=DIMT1 PE=1 SV=1 \\
\hline 49,28 & 6 & Activating signal cointegrator 1 complex subunit $1 \mathrm{OS}=$ Homo sapiens $\mathrm{GN}=\mathrm{ASCC} 1 \mathrm{PE}=2 \mathrm{SV}=1$ \\
\hline 49,05 & 22 & 40S ribosomal protein $\mathrm{SA}$ (Fragment) $\mathrm{OS}=$ Homo sapiens $\mathrm{GN}=\mathrm{RPSA} \mathrm{PE}=3 \mathrm{SV}=1$ \\
\hline 48,11 & 4 & Exosome complex component RRP40 OS=Homo sapiens GN=EXOSC3 PE=1 SV=3 \\
\hline 48,04 & 6 & 60S ribosomal protein $\mathrm{L} 14 \mathrm{OS}=$ Homo sapiens $\mathrm{GN}=\mathrm{RPL} 14 \mathrm{PE}=4 \mathrm{SV}=1$ \\
\hline 46,98 & 4 & Slit homolog 2 protein $\mathrm{OS}=$ Homo sapiens $\mathrm{GN}=\mathrm{SLIT} 2 \mathrm{PE}=1 \mathrm{SV}=1$ \\
\hline 45,93 & 2 & V-set and immunoglobulin domain-containing protein $8 \mathrm{OS}=$ Homo sapiens GN=VSIG8 PE=1 SV=1 \\
\hline 45,91 & 25 & T-complex protein 1 subunit beta $\mathrm{OS}=$ Homo sapiens $\mathrm{GN}=\mathrm{CCT} 2 \mathrm{PE}=1 \mathrm{SV}=4$ \\
\hline 45,53 & 19 & Heterogeneous nuclear ribonucleoprotein $\mathrm{U}$ OS=Homo sapiens GN=HNRNPU PE=1 SV=6 \\
\hline 44,77 & 7 & 60S ribosomal protein L30 (Fragment) OS=Homo sapiens GN=RPL30 PE=2 SV=1 \\
\hline 44,25 & 32 & Splicing factor, proline- and glutamine-rich OS=Homo sapiens GN=SFPQ PE=1 SV=2 \\
\hline 42,75 & 10 & Eukaryotic translation initiation factor 2 subunit $2 \mathrm{OS}=$ Homo sapiens GN=EIF2S2 PE=1 SV=2 \\
\hline 42,32 & 3 & NF-kappa-B-repressing factor OS=Homo sapiens GN=NKRF PE=1 SV=2 \\
\hline 41,37 & 2 & 60S acidic ribosomal protein $\mathrm{P} 1 \mathrm{OS}=$ Homo sapiens $\mathrm{GN}=\mathrm{RPLP} 1 \mathrm{PE}=1 \mathrm{SV}=1$ \\
\hline 41,18 & 29 & Activating signal cointegrator 1 complex subunit $3 \mathrm{OS}=$ Homo sapiens $\mathrm{GN}=\mathrm{ASCC} 3 \mathrm{PE}=1 \mathrm{SV}=3$ \\
\hline 40,81 & 12 & 40S ribosomal protein $\mathrm{S} 9 \mathrm{OS}=$ Homo sapiens $\mathrm{GN}=\mathrm{RPS} 9 \mathrm{PE}=1 \mathrm{SV}=3$ \\
\hline 40,36 & 24 & 40S ribosomal protein $\mathrm{S} 3 \mathrm{a}$ OS=Homo sapiens $\mathrm{GN}=\mathrm{RPS} 3 \mathrm{~A} \mathrm{PE}=1 \mathrm{SV}=2$ \\
\hline 40,26 & 12 & Eukaryotic translation initiation factor 4 gamma $1 \mathrm{OS}=$ Homo sapiens $\mathrm{GN}=\mathrm{EIF} 4 \mathrm{G} 1 \mathrm{PE}=2 \mathrm{SV}=1$ \\
\hline 38,98 & 3 & Serine/threonine-protein kinase MRCK alpha OS=Homo sapiens GN=CDC42BPA PE $=2 \mathrm{SV}=1$ \\
\hline 38,28 & 62 & ATP-dependent RNA helicase A OS=Homo sapiens GN=DHX9 PE=1 SV=4 \\
\hline 38,26 & 4 & General transcription factor $3 \mathrm{C}$ polypeptide $4 \mathrm{OS}=$ Homo sapiens $\mathrm{GN}=\mathrm{GTF} 3 \mathrm{C} 4 \mathrm{PE}=1 \mathrm{SV}=2$ \\
\hline 37,31 & 4 & $5^{\prime}-3^{\prime}$ exoribonuclease $2 \mathrm{OS}=$ Homo sapiens $\mathrm{GN}=\mathrm{XRN} 2 \mathrm{PE}=2 \mathrm{SV}=1$ \\
\hline 37,22 & 16 & Probable E3 ubiquitin-protein ligase MYCBP2 OS=Homo sapiens GN=MYCBP2 PE=2 SV=1 \\
\hline 37,1 & 2 & Heat shock 105kDa/110kDa protein 1, isoform CRA_b OS=Homo sapiens GN=HSPH1 PE=2 SV=1 \\
\hline 36,4 & 2 & Leucine-tRNA ligase, cytoplasmic OS=Homo sapiens GN=LARS PE=2 SV=1 \\
\hline 36,13 & 3 & Isoform 2 of Endoribonuclease Dicer OS=Homo sapiens GN=DICER1 \\
\hline 35,7 & 2 & Exosome complex exonuclease RRP44 OS=Homo sapiens GN=DIS3 PE=1 SV=2 \\
\hline 35,52 & 11 & $60 \mathrm{~S}$ acidic ribosomal protein $\mathrm{P} 2 \mathrm{OS}=$ Homo sapiens $\mathrm{GN}=\mathrm{RPLP} 2 \mathrm{PE}=1 \mathrm{SV}=1$ \\
\hline 34,39 & 12 & Superkiller viralicidic activity 2-like $2 \mathrm{OS}=$ Homo sapiens $\mathrm{GN}=\mathrm{SKIV} 2 \mathrm{~L} 2 \mathrm{PE}=1 \mathrm{SV}=3$ \\
\hline 33,96 & 8 & $60 \mathrm{~S}$ ribosomal protein $\mathrm{L} 11 \mathrm{OS}=$ Homo sapiens $\mathrm{GN}=\mathrm{RPL} 11 \mathrm{PE}=1 \mathrm{SV}=2$ \\
\hline 32,65 & 2 & Keratin, type I cuticular $\mathrm{Ha} 2 \mathrm{OS}=\mathrm{Homo}$ sapiens $\mathrm{GN}=\mathrm{KRT} 32 \mathrm{PE}=1 \mathrm{SV}=3$ \\
\hline 32,63 & 27 & Putative ATP-dependent RNA helicase DHX30 OS=Homo sapiens GN=DHX30 PE=2 SV=1 \\
\hline 32,49 & 2 & mRNA export factor OS=Homo sapiens GN=RAE1 $\mathrm{PE}=1 \mathrm{SV}=1$ \\
\hline 32,21 & 2 & $\mathrm{C} 2$ domain-containing protein $5 \mathrm{OS}=\mathrm{Homo}$ sapiens $\mathrm{GN}=\mathrm{C} 2 \mathrm{CD} 5 \mathrm{PE}=2 \mathrm{SV}=1$ \\
\hline 32,07 & 35 & 60S acidic ribosomal protein $\mathrm{P} 0 \mathrm{OS}=\mathrm{Homo}$ sapiens $\mathrm{GN}=\mathrm{RPLP} 0 \mathrm{PE}=1 \mathrm{SV}=1$ \\
\hline 32,03 & 4 & WD repeat-containing protein $6 \mathrm{OS}=\mathrm{Homo}$ sapiens $\mathrm{GN}=\mathrm{WDR} 6 \mathrm{PE}=2 \mathrm{SV}=1$ \\
\hline 31,99 & 2 & Splicing factor U2AF $35 \mathrm{kDa}$ subunit $\mathrm{OS}=$ Homo sapiens $\mathrm{GN}=\mathrm{U} 2 \mathrm{AF} 1 \mathrm{PE}=1 \mathrm{SV}=3$ \\
\hline 31,57 & 6 & $60 \mathrm{~S}$ ribosomal protein $\mathrm{L} 28 \mathrm{OS}=\mathrm{Homo}$ sapiens $\mathrm{GN}=\mathrm{RPL} 28 \mathrm{PE}=2 \mathrm{SV}=1$ \\
\hline 31,56 & 4 & Pre-mRNA-processing factor $19 \mathrm{OS}=$ Homo sapiens GN=PRPF19 PE=1 SV=1 \\
\hline 31,3 & 7 & 60S ribosomal protein $\mathrm{L} 35 \mathrm{a} \mathrm{OS}=$ Homo sapiens $\mathrm{GN}=\mathrm{RPL} 35 \mathrm{~A} \mathrm{PE}=1 \mathrm{SV}=2$ \\
\hline 31,08 & 2 & General transcription factor $3 \mathrm{C}$ polypeptide $3 \mathrm{OS}=$ Homo sapiens $\mathrm{GN}=\mathrm{GTF} 3 \mathrm{C} 3 \mathrm{PE}=1 \mathrm{SV}=1$ \\
\hline 30,58 & 2 & Ribosome maturation protein SBDS OS=Homo sapiens GN=SBDS PE=1 SV=4 \\
\hline 30,25 & 3 & Pyrroline-5-carboxylate reductase 2 OS=Homo sapiens GN=PYCR2 PE=1 SV=1 \\
\hline 30,08 & 5 & Oxysterol-binding protein-related protein $8 \mathrm{OS}=$ Homo sapiens $\mathrm{GN}=\mathrm{OSBPL} 8 \mathrm{PE}=1 \mathrm{SV}=3$ \\
\hline 29,71 & 13 & ATP-dependent RNA helicase DDX3X OS=Homo sapiens GN=DDX3X PE=1 SV=3 \\
\hline 29,66 & 8 & Nuclease-sensitive element-binding protein $1 \mathrm{OS}=$ Homo sapiens $\mathrm{GN}=\mathrm{YBX} 1 \mathrm{PE}=1 \mathrm{SV}=3$ \\
\hline 29,54 & 2 & Replication factor $\mathrm{C}$ subunit $3 \mathrm{OS}=$ Homo sapiens $\mathrm{GN}=\mathrm{RFC} 3 \mathrm{PE}=1 \mathrm{SV}=2$ \\
\hline 28,84 & 4 & Heat shock $70 \mathrm{kDa}$ protein $4 \mathrm{~L} \mathrm{OS}=$ Homo sapiens $\mathrm{GN}=\mathrm{HSPA} 4 \mathrm{~L} \mathrm{PE}=2 \mathrm{SV}=2$ \\
\hline 28,61 & 4 & Exosome complex component RRP4 OS=Homo sapiens GN=EXOSC2 PE=1 SV=2 \\
\hline 28,43 & 6 & Isoleucine--tRNA ligase, cytoplasmic OS=Homo sapiens GN=IARS PE=1 SV=2 \\
\hline 28,05 & 3 & Structural maintenance of chromosomes protein $\mathrm{OS}=$ Homo sapiens $\mathrm{GN}=\mathrm{SMC} 4 \mathrm{PE}=2 \mathrm{SV}=1$ \\
\hline 27,54 & 2 & $\mathrm{CD} 2$ antigen cytoplasmic tail-binding protein $2 \mathrm{OS}=\mathrm{Homo}$ sapiens $\mathrm{GN}=\mathrm{CD} 2 \mathrm{BP} 2 \mathrm{PE}=1 \mathrm{SV}=1$ \\
\hline 27,44 & 3 & Interferon-related developmental regulator $1 \mathrm{OS}=$ Homo sapiens GN=IFRD1 PE=1 SV=4 \\
\hline 27,23 & 3 & WD repeat-containing protein 18 (Fragment) $\mathrm{OS}=$ Homo sapiens $\mathrm{GN}=\mathrm{WDR} 18 \mathrm{PE}=4 \mathrm{SV}=1$ \\
\hline 26,85 & 7 & $60 \mathrm{~S}$ ribosomal protein $\mathrm{L} 13 \mathrm{OS}=$ Homo sapiens $\mathrm{GN}=\mathrm{RPL} 13 \mathrm{PE}=1 \mathrm{SV}=4$ \\
\hline 26,77 & 14 & $60 \mathrm{~S}$ ribosomal protein $\mathrm{L} 10 \mathrm{OS}=$ Homo sapiens $\mathrm{GN}=\mathrm{RPL} 10 \mathrm{PE}=2 \mathrm{SV}=1$ \\
\hline 26,66 & 5 & Exosome complex component MTR3 OS=Homo sapiens GN=EXOSC6 PE=1 SV=1 \\
\hline 24,77 & 5 & Protein PRRC2A OS=Homo sapiens GN=PRRC2A PE=1 SV=3 \\
\hline 24,22 & 8 & Mitochondrial ribonuclease $P$ protein $1 \mathrm{OS}=$ Homo sapiens $\mathrm{GN}=\mathrm{TRMT} 10 \mathrm{C} \mathrm{PE}=1 \mathrm{SV}=2$ \\
\hline
\end{tabular}




\begin{tabular}{|c|c|c|}
\hline $\mathbf{H} / \mathbf{L}$ & \# & Description \\
\hline 23,39 & 18 & Guanine nucleotide-binding protein subunit beta-2-like $1 \mathrm{OS}=$ Homo sapiens $\mathrm{GN}=\mathrm{GNB} 2 \mathrm{~L} 1 \mathrm{PE}=1 \mathrm{SV}=3$ \\
\hline 23,13 & 6 & 60S ribosomal protein $\mathrm{L} 10 \mathrm{a} \mathrm{OS}=$ Homo sapiens $\mathrm{GN}=\mathrm{RPL} 10 \mathrm{~A} \mathrm{PE}=1 \mathrm{SV}=2$ \\
\hline 23,12 & 3 & Probable ATP-dependent RNA helicase DDX6 OS=Homo sapiens GN=DDX6 PE=1 SV=2 \\
\hline 22,89 & 7 & La-related protein $1 \mathrm{OS}=$ Homo sapiens GN=LARP1 $\mathrm{PE}=1 \mathrm{SV}=2$ \\
\hline 22,74 & 13 & Pre-rRNA-processing protein TSR1 homolog OS=Homo sapiens GN=TSR1 PE=1 SV=1 \\
\hline 22,33 & 45 & Elongation factor $2 \mathrm{OS}=$ Homo sapiens $\mathrm{GN}=\mathrm{EEF} 2 \mathrm{PE}=1 \mathrm{SV}=4$ \\
\hline 21,99 & 2 & Stress-induced-phosphoprotein $1 \mathrm{OS}=$ Homo sapiens GN=STIP1 PE=4 SV=1 \\
\hline 21,57 & 12 & T-complex protein 1 subunit eta $\mathrm{OS}=$ Homo sapiens $\mathrm{GN}=\mathrm{CCT} 7 \mathrm{PE}=1 \mathrm{SV}=2$ \\
\hline 21,4 & 2 & Periodic tryptophan protein 1 homolog OS=Homo sapiens GN=PWP1 PE=2 SV=1 \\
\hline 20,83 & 8 & Dual specificity protein phosphatase $12 \mathrm{OS}=$ Homo sapiens GN=DUSP12 PE=1 SV=1 \\
\hline 20,82 & 2 & Zinc finger CCHC domain-containing protein $8 \mathrm{OS}=$ Homo sapiens GN=ZCCHC8 PE=1 SV=2 \\
\hline 20,71 & 12 & 40S ribosomal protein $\mathrm{S} 8 \mathrm{OS}=$ Homo sapiens $\mathrm{GN}=\mathrm{RPS} 8 \mathrm{PE}=1 \mathrm{SV}=2$ \\
\hline 20,7 & 13 & Eukaryotic translation initiation factor 2 subunit $1 \mathrm{OS}=$ Homo sapiens GN=EIF2S1 PE=1 SV=3 \\
\hline 20,53 & 2 & Proline-, glutamic acid- and leucine-rich protein $1 \mathrm{OS}=$ Homo sapiens GN=PELP1 PE=2 SV=1 \\
\hline 20,03 & 2 & Isoform 2 of Ribosomal biogenesis protein LAS1L OS=Homo sapiens GN=LAS1L \\
\hline 20,01 & 4 & Lupus La protein $\mathrm{OS}=\mathrm{H}$ omo sapiens $\mathrm{GN}=\mathrm{SSB} \mathrm{PE}=1 \mathrm{SV}=2$ \\
\hline 19,97 & 4 & Exosome complex component RRP43 OS=Homo sapiens GN=EXOSC8 PE=1 SV=1 \\
\hline 19,9 & 8 & Eukaryotic translation initiation factor 3 subunit $\mathrm{J}$ OS=Homo sapiens GN=EIF3J PE=1 SV=2 \\
\hline 19,88 & 126 & Vimentin OS=Homo sapiens GN=VIM PE=1 SV=4 \\
\hline 19,46 & 10 & Signal recognition particle $14 \mathrm{kDa}$ protein $\mathrm{OS}=$ Homo sapiens $\mathrm{GN}=\mathrm{SRP} 14 \mathrm{PE}=1 \mathrm{SV}=2$ \\
\hline 19,45 & 3 & 60S ribosomal protein $\mathrm{L} 27 \mathrm{a} \mathrm{OS}=\mathrm{Homo}$ sapiens $\mathrm{GN}=\mathrm{RPL} 27 \mathrm{~A} \mathrm{PE}=2 \mathrm{SV}=1$ \\
\hline 19,32 & 4 & $60 \mathrm{~S}$ ribosomal protein $\mathrm{L} 4 \mathrm{OS}=\mathrm{Homo}$ sapiens $\mathrm{GN}=\mathrm{RPL} 4 \mathrm{PE}=4 \mathrm{SV}=1$ \\
\hline 19,21 & 3 & Stabilin-2 OS=Homo sapiens GN=STAB2 PE $=1 \mathrm{SV}=3$ \\
\hline 19,08 & 4 & Eukaryotic translation initiation factor $6 \mathrm{OS}=$ Homo sapiens GN=EIF6 PE=1 SV=1 \\
\hline 18,95 & 6 & Structural maintenance of chromosomes protein $3 \mathrm{OS}=$ Homo sapiens $\mathrm{GN}=\mathrm{SMC} 3 \mathrm{PE}=1 \mathrm{SV}=2$ \\
\hline 18,93 & 10 & Probable ATP-dependent RNA helicase DDX17 OS=Homo sapiens GN=DDX17 PE=2 SV=1 \\
\hline 18,68 & 11 & $116 \mathrm{kDa}$ U5 small nuclear ribonucleoprotein component OS=Homo sapiens GN=EFTUD2 PE=2 SV=1 \\
\hline 18,51 & 5 & Probable ATP-dependent RNA helicase YTHDC2 OS=Homo sapiens GN=YTHDC2 PE=1 SV=2 \\
\hline 18,47 & 19 & Proliferation-associated protein 2G4 OS=Homo sapiens GN=PA2G4 PE=1 SV=3 \\
\hline 18,14 & 2 & 60S ribosomal protein $\mathrm{L} 35 \mathrm{OS}=$ Homo sapiens $\mathrm{GN}=\mathrm{RPL} 35 \mathrm{PE}=1 \mathrm{SV}=2$ \\
\hline 17,27 & 2 & Methionine--tRNA ligase, cytoplasmic OS=Homo sapiens GN=MARS PE=1 SV=2 \\
\hline 17,26 & 11 & $\mathrm{X}$-ray repair cross-complementing protein $5 \mathrm{OS}=\mathrm{Homo}$ sapiens $\mathrm{GN}=\mathrm{XRCC} 5 \mathrm{PE}=1 \mathrm{SV}=3$ \\
\hline 16,78 & 4 & E3 UFM1-protein ligase 1 OS=Homo sapiens GN=UFL1 PE=1 SV=2 \\
\hline 15,86 & 29 & Eukaryotic translation initiation factor 3 subunit M OS=Homo sapiens GN=EIF3M PE=1 SV=1 \\
\hline 15,84 & 3 & Putative pre-mRNA-splicing factor ATP-dependent RNA helicase DHX15 OS=Homo sapiens GN=DHX15 PE=1 SV=2 \\
\hline 15,63 & 13 & Eukaryotic translation initiation factor 3 subunit $\mathrm{F}$ OS=Homo sapiens GN=EIF3F PE=2 SV=1 \\
\hline 15,5 & 12 & Ubiquitin carboxyl-terminal hydrolase OS=Homo sapiens GN=USP7 PE=2 SV=1 \\
\hline 15,48 & 3 & 60S ribosomal protein $\mathrm{L} 7 \mathrm{a}$ (Fragment) $\mathrm{OS}=$ Homo sapiens $\mathrm{GN}=\mathrm{RPL} 7 \mathrm{~A} \mathrm{PE}=4 \mathrm{SV}=1$ \\
\hline 15,09 & 27 & $60 \mathrm{~S}$ ribosomal protein $\mathrm{L} 5 \mathrm{OS}=$ Homo sapiens $\mathrm{GN}=\mathrm{RPL} 5 \mathrm{PE}=1 \mathrm{SV}=3$ \\
\hline 15,05 & 9 & Pre-mRNA-processing factor $6 \mathrm{OS}=$ Homo sapiens $\mathrm{GN}=\mathrm{PRPF} 6 \mathrm{PE}=1 \mathrm{SV}=1$ \\
\hline 15,04 & 2 & Uncharacterized protein $\mathrm{OS}=\mathrm{Homo}$ sapiens $\mathrm{GN}=\mathrm{QARS} \mathrm{PE}=2 \mathrm{SV}=1$ \\
\hline 14,65 & 2 & $28 \mathrm{~S}$ ribosomal protein $\mathrm{S} 27$, mitochondrial $\mathrm{OS}=$ Homo sapiens $\mathrm{GN}=\mathrm{MRPS} 27 \mathrm{PE}=2 \mathrm{SV}=1$ \\
\hline 14,16 & 5 & Transcription intermediary factor 1-beta OS=Homo sapiens GN=TRIM28 PE=1 SV=5 \\
\hline 14 & 15 & $60 \mathrm{~S}$ ribosomal protein $\mathrm{L} 7 \mathrm{OS}=$ Homo sapiens $\mathrm{GN}=\mathrm{RPL} 7 \mathrm{PE}=3 \mathrm{SV}=1$ \\
\hline 13,85 & 6 & Insulin-like growth factor 2 mRNA-binding protein $1 \mathrm{OS}=$ Homo sapiens GN=IGF2BP1 PE=1 SV=2 \\
\hline 13,79 & 13 & $40 \mathrm{~S}$ ribosomal protein $\mathrm{S} 4, \mathrm{X}$ isoform $\mathrm{OS}=$ Homo sapiens $\mathrm{GN}=\mathrm{RPS} 4 \mathrm{X} \mathrm{PE}=1 \mathrm{SV}=2$ \\
\hline 13,62 & 2 & Pre-mRNA-splicing factor SPF27 OS=Homo sapiens GN=BCAS2 PE=1 SV=1 \\
\hline 13,53 & 11 & F-box only protein $50 \mathrm{OS}=$ Homo sapiens $\mathrm{GN}=\mathrm{NCCRP} 1 \mathrm{PE}=1 \mathrm{SV}=1$ \\
\hline 13,38 & 11 & Glycogen debranching enzyme OS=Homo sapiens $\mathrm{GN}=\mathrm{AGL} \mathrm{PE}=1 \mathrm{SV}=3$ \\
\hline 13,29 & 3 & RRP15-like protein OS=Homo sapiens GN=RRP15 PE=1 SV=2 \\
\hline 13,22 & 13 & Heat shock protein HSP 90 -alpha OS=Homo sapiens $\mathrm{GN}=\mathrm{HSP} 90 \mathrm{AA} 1 \mathrm{PE}=1 \mathrm{SV}=5$ \\
\hline 13,13 & 2 & Neurofilament medium polypeptide OS=Homo sapiens GN=NEFM PE=2 SV=1 \\
\hline 13,13 & 8 & 40S ribosomal protein $\mathrm{S} 2$ (Fragment) OS=Homo sapiens GN=RPS2 PE=3 SV=1 \\
\hline 13,09 & 4 & 60S ribosomal protein L18 (Fragment) OS=Homo sapiens GN=RPL18 PE=3 SV=1 \\
\hline 13,06 & 6 & Small nuclear ribonucleoprotein Sm D2 OS=Homo sapiens GN=SNRPD2 PE=1 SV=1 \\
\hline 13,05 & 2 & E3 ubiquitin-protein ligase UBR5 OS=Homo sapiens GN=UBR5 PE=2 SV=1 \\
\hline 12,76 & 17 & Cellular tumor antigen $\mathrm{p} 53 \mathrm{OS}=\mathrm{Homo}$ sapiens $\mathrm{GN}=\mathrm{TP} 53 \mathrm{PE}=1 \mathrm{SV}=4$ \\
\hline 12,66 & 8 & Casein kinase II subunit alpha OS=Homo sapiens $\mathrm{GN}=\mathrm{CSNK} 2 \mathrm{~A} 1 \mathrm{PE}=2 \mathrm{SV}=1$ \\
\hline 12,59 & 2 & 40S ribosomal protein $\mathrm{S} 28 \mathrm{OS}=$ Homo sapiens $\mathrm{GN}=\mathrm{RPS} 28 \mathrm{PE}=1 \mathrm{SV}=1$ \\
\hline 12,28 & 2 & Exosome complex component RRP42 OS=Homo sapiens GN=EXOSC7 PE=1 SV=3 \\
\hline 11,98 & 7 & tRNA-splicing ligase RtcB homolog OS=Homo sapiens GN=RTCB PE=1 SV=1 \\
\hline 11,62 & 2 & Casein kinase II subunit alpha' OS=Homo sapiens GN=CSNK2A2 PE $=1 \mathrm{SV}=1$ \\
\hline 11,25 & 2 & U2 small nuclear ribonucleoprotein $\mathrm{B}$ " OS=Homo sapiens $\mathrm{GN}=\mathrm{SNRPB} 2 \mathrm{PE}=1 \mathrm{SV}=1$ \\
\hline 11,03 & 13 & Poly [ADP-ribose] polymerase $1 \mathrm{OS}=$ Homo sapiens GN=PARP1 PE=1 SV=4 \\
\hline 10,87 & 7 & Bifunctional glutamate/proline--tRNA ligase $\mathrm{OS}=$ Homo sapiens $\mathrm{GN}=\mathrm{EPRS} \mathrm{PE}=1 \mathrm{SV}=5$ \\
\hline 10,76 & 2 & Rho guanine nucleotide exchange factor $2 \mathrm{OS}=$ Homo sapiens $\mathrm{GN}=\mathrm{ARHGEF} 2 \mathrm{PE}=2 \mathrm{SV}=1$ \\
\hline 10,66 & 2 & Electroneutral sodium bicarbonate exchanger $1 \mathrm{OS}=$ Homo sapiens $\mathrm{GN}=\mathrm{SLC} 4 \mathrm{~A} 8 \mathrm{PE}=1 \mathrm{SV}=1$ \\
\hline
\end{tabular}




\begin{tabular}{|c|c|c|}
\hline $\mathbf{H} / \mathbf{L}$ & \# & Description \\
\hline 10,62 & 3 & 40S ribosomal protein $\mathrm{S} 27 \mathrm{OS}=$ Homo sapiens $\mathrm{GN}=\mathrm{RPS} 27 \mathrm{PE}=4 \mathrm{SV}=1$ \\
\hline 10,6 & 2 & RNA-binding protein $\mathrm{PNO} 1 \mathrm{OS}=$ Homo sapiens $\mathrm{GN}=\mathrm{PNO} 1 \mathrm{PE}=2 \mathrm{SV}=1$ \\
\hline 10,56 & 41 & Non-POU domain-containing octamer-binding protein OS=Homo sapiens GN=NONO PE=1 SV=4 \\
\hline 10,24 & 12 & Heat shock protein HSP 90-beta OS=Homo sapiens GN=HSP90AB1 PE=1 SV=4 \\
\hline 9,711 & 5 & Transcriptional activator protein Pur-alpha $\mathrm{OS}=$ Homo sapiens GN=PURA PE=1 SV=2 \\
\hline 9,509 & 6 & $\mathrm{X}$-ray repair cross-complementing protein $6 \mathrm{OS}=$ Homo sapiens $\mathrm{GN}=\mathrm{XRCC} 6 \mathrm{PE}=1 \mathrm{SV}=2$ \\
\hline 9,421 & 5 & Heat shock $70 \mathrm{kDa}$ protein $1 \mathrm{~A} / 1 \mathrm{~B}$ OS=Homo sapiens $\mathrm{GN}=\mathrm{HSPA} 1 \mathrm{~A} \mathrm{PE}=1 \mathrm{SV}=5$ \\
\hline 9,344 & 3 & Ribose-phosphate pyrophosphokinase $1 \mathrm{OS}=$ Homo sapiens GN=PRPS1 PE=1 SV=2 \\
\hline 9,323 & 5 & $60 \mathrm{~S}$ ribosomal protein $\mathrm{L} 38 \mathrm{OS}=$ Homo sapiens $\mathrm{GN}=\mathrm{RPL} 38 \mathrm{PE}=1 \mathrm{SV}=2$ \\
\hline 9,164 & 8 & 60S ribosomal protein L6 OS=Homo sapiens GN=RPL6 PE=1 SV=3 \\
\hline 9,007 & 5 & UPF0568 protein C14orf166 OS=Homo sapiens GN=C14orf166 PE=1 SV=1 \\
\hline 8,808 & 4 & $28 \mathrm{~S}$ ribosomal protein $\mathrm{S} 22$, mitochondrial $\mathrm{OS}=$ Homo sapiens $\mathrm{GN}=\mathrm{MRPS} 22 \mathrm{PE}=4 \mathrm{SV}=1$ \\
\hline 8,753 & 9 & T-complex protein 1 subunit zeta $\mathrm{OS}=$ Homo sapiens $\mathrm{GN}=\mathrm{CCT} 6 \mathrm{~A} \mathrm{PE}=1 \mathrm{SV}=3$ \\
\hline 8,616 & 3 & mRNA turnover protein 4 homolog $\mathrm{OS}=$ Homo sapiens $\mathrm{GN}=\mathrm{MRTO} 4 \mathrm{PE}=1 \mathrm{SV}=2$ \\
\hline 8,555 & 6 & Coiled-coil domain-containing protein $124 \mathrm{OS}=$ Homo sapiens $\mathrm{GN}=\mathrm{CCDC} 124 \mathrm{PE}=1 \mathrm{SV}=1$ \\
\hline 8,263 & 2 & $60 \mathrm{~S}$ ribosomal protein $\mathrm{L} 29 \mathrm{OS}=$ Homo sapiens $\mathrm{GN}=\mathrm{RPL} 29 \mathrm{PE}=1 \mathrm{SV}=2$ \\
\hline 8,145 & 3 & Aminoacyl tRNA synthase complex-interacting multifunctional protein $1 \mathrm{OS}=$ Homo sapiens $\mathrm{GN}=\mathrm{AIMP} 1 \mathrm{PE}=1 \mathrm{SV}=2$ \\
\hline 7,847 & 6 & Gamma-tubulin complex component $2 \mathrm{OS}=$ Homo sapiens GN=TUBGCP2 PE=1 SV=2 \\
\hline 7,719 & 4 & T-complex protein 1 subunit epsilon $\mathrm{OS}=$ Homo sapiens $\mathrm{GN}=\mathrm{CCT} 5 \mathrm{PE}=2 \mathrm{SV}=1$ \\
\hline 7,719 & 4 & Serine-threonine kinase receptor-associated protein OS=Homo sapiens GN=STRAP PE $=2 \mathrm{SV}=1$ \\
\hline 7,662 & 3 & Coatomer subunit alpha $\mathrm{OS}=$ Homo sapiens $\mathrm{GN}=\mathrm{COPA} \mathrm{PE}=1 \mathrm{SV}=2$ \\
\hline 7,617 & 8 & Cytoskeleton-associated protein $4 \mathrm{OS}=$ Homo sapiens $\mathrm{GN}=\mathrm{CKAP} 4 \mathrm{PE}=1 \mathrm{SV}=2$ \\
\hline 7,549 & 3 & Peroxiredoxin-4 OS=Homo sapiens GN=PRDX4 PE=1 SV=1 \\
\hline 7,48 & 4 & Elongation factor 1-gamma OS=Homo sapiens $\mathrm{GN}=\mathrm{EEF} 1 \mathrm{G} \mathrm{PE}=2 \mathrm{SV}=1$ \\
\hline 7,48 & 40 & 40S ribosomal protein $\mathrm{S} 3 \mathrm{OS}=$ Homo sapiens $\mathrm{GN}=\mathrm{RPS} 3 \mathrm{PE}=1 \mathrm{SV}=2$ \\
\hline 7,317 & 12 & $60 \mathrm{~S}$ ribosomal protein $\mathrm{L} 8 \mathrm{OS}=$ Homo sapiens $\mathrm{GN}=\mathrm{RPL} 8 \mathrm{PE}=1 \mathrm{SV}=2$ \\
\hline 7,201 & 3 & Uncharacterized protein $\mathrm{OS}=$ Homo sapiens $\mathrm{GN}=\mathrm{DDB} 1 \mathrm{PE}=4 \mathrm{SV}=1$ \\
\hline 7,15 & 2 & Barrier-to-autointegration factor OS=Homo sapiens $\mathrm{GN}=\mathrm{BANF} 1 \mathrm{PE}=1 \mathrm{SV}=1$ \\
\hline 6,692 & 2 & Chromosome transmission fidelity protein 8 homolog (Fragment) OS=Homo sapiens GN=CHTF8 PE=4 SV=1 \\
\hline 6,614 & 3 & RuvB-like 2 OS=Homo sapiens GN=RUVBL2 PE=1 SV=3 \\
\hline 6,453 & 3 & Nucleosome assembly protein 1-like $1 \mathrm{OS}=$ Homo sapiens GN=NAP1L1 PE=2 SV=1 \\
\hline 6,428 & 7 & 40S ribosomal protein $\mathrm{S} 6 \mathrm{OS}=$ Homo sapiens $\mathrm{GN}=\mathrm{RPS} 6 \mathrm{PE}=1 \mathrm{SV}=1$ \\
\hline 6,14 & 2 & Partner of Y14 and mago OS=Homo sapiens GN=WIBG PE=1 SV=1 \\
\hline 6,077 & 21 & Heat shock cognate $71 \mathrm{kDa}$ protein OS=Homo sapiens GN=HSPA8 PE=1 SV=1 \\
\hline 5,97 & 8 & $60 \mathrm{~S}$ ribosomal protein $\mathrm{L} 37 \mathrm{a} \mathrm{OS}=\mathrm{Homo}$ sapiens $\mathrm{GN}=\mathrm{RPL} 37 \mathrm{~A} \mathrm{PE}=1 \mathrm{SV}=2$ \\
\hline 5,959 & 4 & 40S ribosomal protein $\mathrm{S} 21 \mathrm{OS}=$ Homo sapiens $\mathrm{GN}=\mathrm{RPS} 21 \mathrm{PE}=3 \mathrm{SV}=1$ \\
\hline 5,866 & 9 & Probable ATP-dependent RNA helicase DDX5 OS=Homo sapiens GN=DDX5 PE=2 SV=1 \\
\hline 5,716 & 3 & Replication protein A $32 \mathrm{kDa}$ subunit $\mathrm{OS}=$ Homo sapiens $\mathrm{GN}=\mathrm{RPA} 2 \mathrm{PE}=1 \mathrm{SV}=1$ \\
\hline 5,607 & 5 & Ankyrin repeat domain-containing protein $16 \mathrm{OS}=$ Homo sapiens $\mathrm{GN}=\mathrm{ANKRD} 16 \mathrm{PE}=1 \mathrm{SV}=1$ \\
\hline 5,552 & 5 & RuvB-like $1 \mathrm{OS}=$ Homo sapiens GN=RUVBL1 PE $=1 \mathrm{SV}=1$ \\
\hline 5,527 & 10 & DNA-dependent protein kinase catalytic subunit OS=Homo sapiens GN=PRKDC PE=1 SV=3 \\
\hline 5,508 & 2 & ADP/ATP translocase 3 OS=Homo sapiens GN=SLC25A6 PE=1 SV=4 \\
\hline 5,405 & 3 & Serine/threonine-protein phosphatase PGAM5, mitochondrial OS=Homo sapiens GN=PGAM5 PE=1 SV=2 \\
\hline 5,397 & 3 & Eukaryotic translation initiation factor 5B OS=Homo sapiens GN=EIF5B PE=1 SV=4 \\
\hline 5,074 & 6 & Emerin $\mathrm{OS}=$ Homo sapiens $\mathrm{GN}=\mathrm{EMD} \mathrm{PE}=1 \mathrm{SV}=1$ \\
\hline 4,864 & 4 & Single-stranded DNA-binding protein, mitochondrial OS=Homo sapiens GN=SSBP1 PE=1 SV=1 \\
\hline 4,845 & 4 & 60S ribosomal protein L34 OS=Homo sapiens GN=RPL34 PE=1 SV=3 \\
\hline 4,829 & 2 & TATA-binding protein-associated factor $2 \mathrm{~N}$ OS=Homo sapiens $\mathrm{GN}=\mathrm{TAF} 15 \mathrm{PE}=2 \mathrm{SV}=1$ \\
\hline 4,79 & 2 & Small nuclear ribonucleoprotein Sm D3 OS=Homo sapiens GN=SNRPD3 PE=2 SV=1 \\
\hline 4,754 & 7 & Probable ATP-dependent RNA helicase DDX20 OS=Homo sapiens GN=DDX20 PE=1 SV=2 \\
\hline 4,702 & 56 & Keratin, type II cytoskeletal $78 \mathrm{OS}=$ Homo sapiens $\mathrm{GN}=\mathrm{KRT} 78 \mathrm{PE}=2 \mathrm{SV}=2$ \\
\hline 4,697 & 2 & Extracellular glycoprotein lacritin OS=Homo sapiens GN=LACRT PE=1 SV=1 \\
\hline 4,63 & 3 & Heterogeneous nuclear ribonucleoprotein $\mathrm{A} 3 \mathrm{OS}=$ Homo sapiens GN=HNRNPA3 PE=1 SV=2 \\
\hline 4,627 & 2 & Citron Rho-interacting kinase (Fragment) OS=Homo sapiens GN=CIT PE=4 SV=1 \\
\hline 4,575 & 2 & Sequestosome-1 OS=Homo sapiens GN=SQSTM1 PE=2 SV=1 \\
\hline 4,573 & 2 & Interferon-related IFRD2 (PC4-B) protein OS=Homo sapiens GN=IFRD2 PE=2 SV=1 \\
\hline 4,563 & 2 & THO complex subunit $4 \mathrm{OS}=$ Homo sapiens $\mathrm{GN}=\mathrm{ALYREF} \mathrm{PE}=2 \mathrm{SV}=1$ \\
\hline 4,459 & 2 & Heterogeneous nuclear ribonucleoprotein F OS=Homo sapiens GN=HNRNPF PE=1 SV=3 \\
\hline 4,309 & 2 & Heterogeneous nuclear ribonucleoprotein A0 OS=Homo sapiens GN=HNRNPA0 PE=1 SV=1 \\
\hline 4,295 & 3 & Histone-binding protein RBBP4 OS=Homo sapiens $\mathrm{GN}=\mathrm{RBBP} 4 \mathrm{PE}=1 \mathrm{SV}=3$ \\
\hline 4,275 & 194 & Desmoplakin OS=Homo sapiens GN=DSP PE $=1 \mathrm{SV}=3$ \\
\hline 4,239 & 4 & S-phase kinase-associated protein $1 \mathrm{OS}=\mathrm{Homo}$ sapiens $\mathrm{GN}=\mathrm{SKP} 1 \mathrm{PE}=2 \mathrm{SV}=1$ \\
\hline 4,147 & 2 & Serine protease HTRA3 OS=Homo sapiens GN=HTRA3 PE=1 SV=2 \\
\hline 4,085 & 3 & Dual-specificity protein phosphatase 3 (Fragment) OS=Homo sapiens GN=DUSP3 PE=4 SV=1 \\
\hline 4,053 & 13 & CAD protein $\mathrm{OS}=$ Homo sapiens $\mathrm{GN}=\mathrm{CAD} \mathrm{PE}=2 \mathrm{SV}=1$ \\
\hline 3,909 & 2 & C-1-tetrahydrofolate synthase, cytoplasmic OS=Homo sapiens GN=MTHFD1 PE=2 SV=1 \\
\hline 3,87 & 6 & Tubulin beta chain $\mathrm{OS}=$ Homo sapiens $\mathrm{GN}=\mathrm{TUBB} \mathrm{PE}=4 \mathrm{SV}=1$ \\
\hline
\end{tabular}




\begin{tabular}{|c|c|c|}
\hline $\mathbf{H} / \mathbf{L}$ & \# & Description \\
\hline 3,852 & 2 & Tubulin beta- $4 \mathrm{~A}$ chain $\mathrm{OS}=$ Homo sapiens $\mathrm{GN}=\mathrm{TUBB} 4 \mathrm{~A} \mathrm{PE}=1 \mathrm{SV}=2$ \\
\hline 3,828 & 9 & Histone deacetylase $6 \mathrm{OS}=$ Homo sapiens $\mathrm{GN}=\mathrm{HDAC} 6 \mathrm{PE}=2 \mathrm{SV}=1$ \\
\hline 3,821 & 7 & Polyadenylate-binding protein $1 \mathrm{OS}=$ Homo sapiens $\mathrm{GN}=\mathrm{PABPC} 1 \mathrm{PE}=2 \mathrm{SV}=1$ \\
\hline 3,741 & 8 & Gem-associated protein $4 \mathrm{OS}=$ Homo sapiens $\mathrm{GN}=\mathrm{GEMIN} 4 \mathrm{PE}=2 \mathrm{SV}=1$ \\
\hline 3,727 & 2 & RNA-binding motif protein, $\mathrm{X}$ chromosome $\mathrm{OS}=$ Homo sapiens $\mathrm{GN}=\mathrm{RBMX} \mathrm{PE}=1 \mathrm{SV}=3$ \\
\hline 3,694 & 2 & Tubulin alpha-4A chain OS=Homo sapiens GN=TUBA4A PE=2 SV=1 \\
\hline 3,671 & 3 & ADP/ATP translocase 2 OS=Homo sapiens $\mathrm{GN}=\mathrm{SLC} 25 \mathrm{~A} 5 \mathrm{PE}=1 \mathrm{SV}=7$ \\
\hline 3,621 & 3 & Phosphate carrier protein, mitochondrial OS=Homo sapiens GN=SLC25A3 PE $=1 \mathrm{SV}=2$ \\
\hline 3,617 & 2 & Gem-associated protein $6 \mathrm{OS}=$ Homo sapiens GN=GEMIN6 PE=1 SV=1 \\
\hline 3,611 & 7 & Heterogeneous nuclear ribonucleoproteins $\mathrm{C} 1 / \mathrm{C} 2 \mathrm{OS}=$ Homo sapiens $\mathrm{GN}=\mathrm{HNRNPC} \mathrm{PE}=2 \mathrm{SV}=1$ \\
\hline 3,609 & 2 & Survival motor neuron protein $\mathrm{OS}=$ Homo sapiens $\mathrm{GN}=\mathrm{SMN} 1 \mathrm{PE}=4 \mathrm{SV}=1$ \\
\hline 3,515 & 5 & Heterogeneous nuclear ribonucleoprotein $\mathrm{A} 1 \mathrm{OS}=$ Homo sapiens $\mathrm{GN}=\mathrm{HNRNPA} 1 \mathrm{PE}=4 \mathrm{SV}=2$ \\
\hline 3,411 & 2 & Kallikrein-7 (Fragment) OS=Homo sapiens GN=KLK7 PE=2 SV=1 \\
\hline 3,398 & 2 & Tubulin beta- 6 chain $\mathrm{OS}=$ Homo sapiens $\mathrm{GN}=\mathrm{TUBB} 6 \mathrm{PE}=1 \mathrm{SV}=1$ \\
\hline 3,38 & 6 & Splicing factor $3 \mathrm{~B}$ subunit $3 \mathrm{OS}=$ Homo sapiens $\mathrm{GN}=\mathrm{SF} 3 \mathrm{~B} 3 \mathrm{PE}=1 \mathrm{SV}=4$ \\
\hline 3,308 & 2 & ELAV-like protein $1 \mathrm{OS}=$ Homo sapiens GN=ELAVL1 $\mathrm{PE}=2 \mathrm{SV}=1$ \\
\hline 3,228 & 2 & Apolipoprotein A-I OS=Homo sapiens $\mathrm{GN}=\mathrm{APOA} 1 \mathrm{PE}=1 \mathrm{SV}=1$ \\
\hline 3,222 & 3 & Neuroblast differentiation-associated protein AHNAK OS=Homo sapiens GN=AHNAK PE=1 SV=2 \\
\hline 3,216 & 2 & Arf-GAP with dual PH domain-containing protein 2 (Fragment) $\mathrm{OS}=$ Homo sapiens $\mathrm{GN}=\mathrm{ADAP} 2 \mathrm{PE}=4 \mathrm{SV}=1$ \\
\hline 3,198 & 3 & Mitochondrial dicarboxylate carrier OS=Homo sapiens GN=SLC25A10 PE=2 SV=1 \\
\hline 3,198 & 10 & BPI fold-containing family A member 2 OS=Homo sapiens GN=BPIFA2 PE=1 SV=2 \\
\hline 3,17 & 3 & Poly(A) binding protein, cytoplasmic 4 (Inducible form), isoform CRA_e OS=Homo sapiens GN=PABPC4 PE=4 SV=1 \\
\hline 3,154 & 2 & Protein arginine N-methyltransferase $1 \mathrm{OS}=$ Homo sapiens GN=PRMT1 PE=4 SV=1 \\
\hline 3,139 & 5 & Heterogeneous nuclear ribonucleoprotein $\mathrm{H}$ OS=Homo sapiens GN=HNRNPH1 PE=2 SV=1 \\
\hline 3,107 & 3 & $60 \mathrm{~S}$ ribosomal protein L32 (Fragment) OS=Homo sapiens GN=RPL32 PE=2 SV=1 \\
\hline 3,015 & 3 & Proteasome subunit alpha type OS=Homo sapiens GN=PSMA6 PE=3 SV=1 \\
\hline 2,976 & 3 & 14-3-3 protein epsilon $\mathrm{OS}=$ Homo sapiens $\mathrm{GN}=\mathrm{YWHAE} \mathrm{PE}=1 \mathrm{SV}=1$ \\
\hline 2,954 & 3 & Sodium/potassium-transporting ATPase subunit alpha-1 OS=Homo sapiens GN=ATP1A1 PE=1 SV=1 \\
\hline 2,932 & 8 & T-complex protein 1 subunit delta $\mathrm{OS}=$ Homo sapiens $\mathrm{GN}=\mathrm{CCT} 4 \mathrm{PE}=1 \mathrm{SV}=4$ \\
\hline 2,93 & 2 & Delta(3,5)-Delta(2,4)-dienoyl-CoA isomerase, mitochondrial OS=Homo sapiens GN=ECH1 PE=1 SV=2 \\
\hline 2,887 & 4 & Ankyrin repeat domain-containing protein $34 \mathrm{C}$ OS=Homo sapiens $\mathrm{GN}=\mathrm{ANKRD} 34 \mathrm{C} \mathrm{PE}=2 \mathrm{SV}=2$ \\
\hline 2,882 & 4 & D-3-phosphoglycerate dehydrogenase OS=Homo sapiens GN=PHGDH PE=1 SV=4 \\
\hline 2,823 & 3 & Gamma-glutamyl hydrolase OS=Homo sapiens GN=GGH PE=1 SV=2 \\
\hline 2,801 & 3 & Carboxypeptidase A4 OS=Homo sapiens GN=CPA4 PE $=2 \mathrm{SV}=1$ \\
\hline 2,742 & 68 & Zymogen granule protein 16 homolog B OS=Homo sapiens $\mathrm{GN}=\mathrm{ZG16B} P E=1 \mathrm{SV}=3$ \\
\hline 2,707 & 6 & DnaJ homolog subfamily A member $1 \mathrm{OS}=$ Homo sapiens GN=DNAJA1 PE=1 SV=2 \\
\hline 2,682 & 7 & Plakophilin-1 OS=Homo sapiens GN=PKP1 PE=1 SV=2 \\
\hline 2,679 & 3 & PHD finger protein $6 \mathrm{OS}=$ Homo sapiens $\mathrm{GN}=\mathrm{PHF} 6 \mathrm{PE}=2 \mathrm{SV}=1$ \\
\hline 2,643 & 2 & Ubiquitin-like modifier-activating enzyme $6 \mathrm{OS}=$ Homo sapiens $\mathrm{GN}=\mathrm{UBA} 6 \mathrm{PE}=1 \mathrm{SV}=1$ \\
\hline 2,597 & 2 & Replication factor $\mathrm{C}$ subunit $5 \mathrm{OS}=\mathrm{Homo}$ sapiens $\mathrm{GN}=\mathrm{RFC} 5 \mathrm{PE}=1 \mathrm{SV}=1$ \\
\hline 2,582 & 2 & SPATA21 protein OS=Homo sapiens $\mathrm{GN}=\mathrm{SPATA} 21 \mathrm{PE}=2 \mathrm{SV}=1$ \\
\hline 2,56 & 549 & Keratin, type I cytoskeletal $9 \mathrm{OS}=$ Homo sapiens $\mathrm{GN}=\mathrm{KRT} 9 \mathrm{PE}=1 \mathrm{SV}=3$ \\
\hline 2,473 & 2 & Cystatin-SA OS=Homo sapiens GN=CST2 PE $=1 \mathrm{SV}=1$ \\
\hline 2,458 & 2 & Filamin-A-interacting protein $1 \mathrm{OS}=$ Homo sapiens $\mathrm{GN}=\mathrm{FILIP} 1 \mathrm{PE}=2 \mathrm{SV}=1$ \\
\hline 2,444 & 3 & Histone H1.4 OS=Homo sapiens GN=HIST1H1E PE=1 SV=2 \\
\hline 2,4 & 2 & UPF0762 protein C6orf58 OS=Homo sapiens GN=C6orf58 $\mathrm{PE}=1 \mathrm{SV}=2$ \\
\hline 2,395 & 19 & Tubulin alpha-1A chain OS=Homo sapiens GN=TUBA1A PE=1 SV=1 \\
\hline 2,383 & 5 & Estradiol 17-beta-dehydrogenase 12 OS=Homo sapiens GN=HSD17B12 PE=1 SV=2 \\
\hline 2,38 & 19 & Catalase $\mathrm{OS}=$ Homo sapiens $\mathrm{GN}=\mathrm{CAT} \mathrm{PE}=1 \mathrm{SV}=3$ \\
\hline 2,339 & 2 & Ig kappa chain V-III region SIE OS=Homo sapiens $\mathrm{PE}=1 \mathrm{SV}=1$ \\
\hline 2,315 & 20 & $78 \mathrm{kDa}$ glucose-regulated protein $\mathrm{OS}=\mathrm{Homo}$ sapiens $\mathrm{GN}=\mathrm{HSPA} 5 \mathrm{PE}=1 \mathrm{SV}=2$ \\
\hline 2,295 & 4 & ATP-binding cassette sub-family A member $10 \mathrm{OS}=$ Homo sapiens $\mathrm{GN}=\mathrm{ABCA} 10 \mathrm{PE}=3 \mathrm{SV}=1$ \\
\hline 2,277 & 12 & Lipocalin-1 OS=Homo sapiens GN=LCN1 PE=1 SV=1 \\
\hline 2,275 & 10 & Keratin, type II cytoskeletal $80 \mathrm{OS}=$ Homo sapiens $\mathrm{GN}=\mathrm{KRT} 80 \mathrm{PE}=1 \mathrm{SV}=2$ \\
\hline 2,268 & 19 & Arginase-1 OS=Homo sapiens GN=ARG1 PE $=1 \mathrm{SV}=2$ \\
\hline 2,258 & 11 & Keratin, type II cytoskeletal 72 OS=Homo sapiens GN=KRT72 PE=1 SV=2 \\
\hline 2,249 & 2 & Isoform 9 of Deleted in malignant brain tumors 1 protein OS=Homo sapiens GN=DMBT1 \\
\hline 2,247 & 4 & Immunoglobulin J chain (Fragment) OS=Homo sapiens GN=IGJ PE=2 SV=1 \\
\hline 2,228 & 77 & Caspase-14 OS=Homo sapiens GN=CASP14 PE $=1 \mathrm{SV}=2$ \\
\hline 2,215 & 641 & Keratin, type II cytoskeletal $1 \mathrm{OS}=$ Homo sapiens GN=KRT1 PE=1 SV=6 \\
\hline 2,2 & 2 & Histone $\mathrm{H} 2 \mathrm{~A}$ type $1 \mathrm{OS}=\mathrm{Homo}$ sapiens $\mathrm{GN}=\mathrm{HIST} 1 \mathrm{H} 2 \mathrm{AG} \mathrm{PE}=1 \mathrm{SV}=2$ \\
\hline 2,189 & 4 & LVV-hemorphin-7 (Fragment) OS=Homo sapiens GN=HBB PE=2 SV=1 \\
\hline 2,155 & 2 & Protein polybromo-1 OS=Homo sapiens $\mathrm{GN}=\mathrm{PBRM} 1 \mathrm{PE}=1 \mathrm{SV}=1$ \\
\hline 2,146 & 2 & Elongation factor 1-delta (Fragment) OS=Homo sapiens GN=EEF1D PE=4 SV=1 \\
\hline 2,109 & 2 & Myosin light polypeptide 6 OS=Homo sapiens GN=MYL6 PE=4 SV=1 \\
\hline 2,1 & 3 & Heterogeneous nuclear ribonucleoprotein U-like protein 1 (Fragment) OS=Homo sapiens GN=HNRNPUL1 PE=4 SV=1 \\
\hline 2,099 & 3 & Trypsin-1 OS=Homo sapiens GN=PRSS1 PE=2 SV=1 \\
\hline
\end{tabular}




\begin{tabular}{|c|c|c|}
\hline $\mathbf{H} / \mathbf{L}$ & \# & Description \\
\hline 2,087 & 2 & ER lumen protein retaining receptor $2 \mathrm{OS}=$ Homo sapiens $\mathrm{GN}=\mathrm{KDELR} 2 \mathrm{PE}=1 \mathrm{SV}=1$ \\
\hline 2,085 & 2 & Vacuolar protein sorting-associated protein 72 homolog OS=Homo sapiens GN=VPS72 PE=1 SV=1 \\
\hline 2,07 & 4 & Cystatin-M OS=Homo sapiens GN=CST6 PE $=1 \mathrm{SV}=1$ \\
\hline 2,067 & 2 & Cullin-3 OS=Homo sapiens GN=CUL3 PE $=1 \mathrm{SV}=2$ \\
\hline 2,062 & 447 & Keratin, type II cytoskeletal 2 epidermal OS=Homo sapiens GN=KRT2 PE=1 SV=2 \\
\hline 2,058 & 4 & Serpin B5 OS=Homo sapiens GN=SERPINB5 PE=1 SV=2 \\
\hline 2,057 & 11 & ATPase family AAA domain-containing protein $3 \mathrm{C} \mathrm{OS}=$ Homo sapiens $\mathrm{GN}=\mathrm{ATAD} 3 \mathrm{C} \mathrm{PE}=2 \mathrm{SV}=2$ \\
\hline 2,043 & 5 & Ig lambda-2 chain $\mathrm{C}$ regions $\mathrm{OS}=\mathrm{Homo}$ sapiens $\mathrm{GN}=\mathrm{IGLC} 2 \mathrm{PE}=1 \mathrm{SV}=1$ \\
\hline 2,04 & 70 & Keratin, type II cytoskeletal $1 \mathrm{~b}$ OS=Homo sapiens GN=KRT77 PE=2 SV=3 \\
\hline 2,033 & 2 & Uncharacterized protein (Fragment) $\mathrm{OS}=$ Homo sapiens $\mathrm{PE}=4 \mathrm{SV}=1$ \\
\hline 2,025 & 15 & Fatty acid synthase OS=Homo sapiens GN=FASN PE $=1 \mathrm{SV}=3$ \\
\hline 2,016 & 13 & Gasdermin-A (Fragment) OS=Homo sapiens GN=GSDMA PE $=4 \mathrm{SV}=1$ \\
\hline 2,001 & 15 & SERPINB12 protein OS=Homo sapiens GN=SERPINB12 $\mathrm{PE}=2 \mathrm{SV}=1$ \\
\hline 1,991 & 41 & Dermcidin OS=Homo sapiens GN=DCD PE $=1 \mathrm{SV}=2$ \\
\hline 1,989 & 6 & Cystatin-S OS=Homo sapiens GN=CST4 PE $=1 \mathrm{SV}=3$ \\
\hline 1,984 & 2 & Putative elongation factor 1-alpha-like 3 OS=Homo sapiens GN=EEF1A1P5 PE=5 SV=1 \\
\hline 1,984 & 427 & Keratin, type I cytoskeletal 10 OS=Homo sapiens GN=KRT10 PE=1 SV=6 \\
\hline 1,976 & 2 & HERV-K_5q33.3 provirus ancestral Pol protein OS=Homo sapiens $\mathrm{PE}=3 \mathrm{SV}=2$ \\
\hline 1,974 & 35 & Filaggrin OS=Homo sapiens GN=FLG PE $=1 \mathrm{SV}=3$ \\
\hline 1,949 & 4 & Alpha-actinin-4 OS=Homo sapiens $\mathrm{GN}=\mathrm{ACTN} 4 \mathrm{PE}=1 \mathrm{SV}=2$ \\
\hline 1,942 & 23 & Bleomycin hydrolase $\mathrm{OS}=$ Homo sapiens $\mathrm{GN}=\mathrm{BLMH} \mathrm{PE}=1 \mathrm{SV}=1$ \\
\hline 1,941 & 23 & Fatty acid-binding protein, epidermal OS=Homo sapiens GN=FABP5 PE=1 SV=3 \\
\hline 1,917 & 4 & Lysosomal protective protein $\mathrm{OS}=$ Homo sapiens $\mathrm{GN}=\mathrm{CTSA} \mathrm{PE}=1 \mathrm{SV}=2$ \\
\hline 1,914 & 3 & Complement component $1 \mathrm{Q}$ subcomponent-binding protein, mitochondrial OS=Homo sapiens GN=C1QBP PE $=1 \mathrm{SV}=1$ \\
\hline 1,899 & 31 & Cystatin-A OS=Homo sapiens GN=CSTA PE=1 SV=1 \\
\hline 1,887 & 3 & Keratin, type II cuticular Hb6 OS=Homo sapiens GN=KRT86 PE=1 SV=1 \\
\hline 1,878 & 2 & Protein LOC101927453 OS=Homo sapiens GN=LOC101927453 PE=4 SV=1 \\
\hline 1,864 & 141 & Hornerin OS $=$ Homo sapiens GN=HRNR PE $=1 \mathrm{SV}=2$ \\
\hline 1,863 & 2 & Tubulin epsilon chain $\mathrm{OS}=\mathrm{Homo}$ sapiens $\mathrm{GN}=\mathrm{TUBE} 1 \mathrm{PE}=3 \mathrm{SV}=1$ \\
\hline 1,862 & 2 & Neuralized-like protein 4 (Fragment) OS=Homo sapiens GN=NEURL4 PE=4 SV=1 \\
\hline 1,851 & 2 & Envoplakin OS=Homo sapiens GN=EVPL PE $=2 \mathrm{SV}=1$ \\
\hline 1,839 & 7 & Cystatin-SN OS=Homo sapiens GN=CST1 PE $=1 \mathrm{SV}=3$ \\
\hline
\end{tabular}

\subsubsection{PI4K2 $\alpha$ binding partners identified by mass spectrometry}

\section{Table 11: PI4K2 $\alpha$ binding partner identified by MS/MS-based mass spectrometry}

Only MS/MS-results with enrichment in the heavy metal labelled fraction are listed here. The threshold was arbitrarily set to $\mathrm{H} / \mathrm{L}>1.8$. Experiments were performed in collaboration with Eberhard Krause. H/L - heavy/ light ratio; \# - number of unique peptides

\begin{tabular}{|c|c|c|}
\hline $\mathrm{H} / \mathrm{L}$ & \# & Description \\
\hline $1,99 * 10^{5}$ & 4 & Leucine-rich repeat-containing protein 14B OS=Homo sapiens GN=LRRC14B PE=3 SV=3 \\
\hline $4,81 * 10^{4}$ & 3 & DnaJ homolog subfamily $\mathrm{C}$ member $10 \mathrm{OS}=$ Homo sapiens GN=DNAJC10 PE=1 SV=2 \\
\hline 1019 & 4 & Protein FAM149A OS=Homo sapiens GN=FAM149A PE=2 SV=2 \\
\hline 499,2 & 3 & Peptidyl-prolyl cis-trans isomerase FKBP8 OS=Homo sapiens $\mathrm{GN}=\mathrm{FKBP} 8 \mathrm{PE}=2 \mathrm{SV}=1$ \\
\hline 264,5 & 34 & T-complex protein 1 subunit alpha $\mathrm{OS}=$ Homo sapiens $\mathrm{GN}=\mathrm{TCP} 1 \mathrm{PE}=1 \mathrm{SV}=1$ \\
\hline 247,9 & 32 & T-complex protein 1 subunit gamma $\mathrm{OS}=$ Homo sapiens $\mathrm{GN}=\mathrm{CCT} 3 \mathrm{PE}=1 \mathrm{SV}=4$ \\
\hline 205,3 & 5 & MMS19 nucleotide excision repair protein homolog OS=Homo sapiens GN=MMS19 PE $=1 \mathrm{SV}=2$ \\
\hline 203 & 44 & T-complex protein 1 subunit theta $\mathrm{OS}=$ Homo sapiens $\mathrm{GN}=\mathrm{CCT} 8 \mathrm{PE}=1 \mathrm{SV}=4$ \\
\hline 182,7 & 210 & Phosphatidylinositol 4-kinase type 2-alpha OS=Homo sapiens GN=PI4K2A PE=1 SV=1 \\
\hline 151,7 & 2 & Telomere length regulation protein TEL2 homolog OS=Homo sapiens GN=TELO2 $\mathrm{PE}=1 \mathrm{SV}=2$ \\
\hline 150,2 & 2 & FAST kinase domain-containing protein $5 \mathrm{OS}=$ Homo sapiens $\mathrm{GN}=\mathrm{FASTKD} 5 \mathrm{PE}=1 \mathrm{SV}=1$ \\
\hline 149 & 19 & T-complex protein 1 subunit zeta $\mathrm{OS}=$ Homo sapiens $\mathrm{GN}=\mathrm{CCT} 6 \mathrm{~A} \mathrm{PE}=1 \mathrm{SV}=3$ \\
\hline 142,3 & 19 & DnaJ homolog subfamily A member $1 \mathrm{OS}=$ Homo sapiens GN=DNAJA1 PE=1 SV=2 \\
\hline 141,2 & 3 & Importin-5 (Fragment) OS=Homo sapiens GN=IPO5 PE=4 SV=1 \\
\hline 128,2 & 3 & Endoplasmic reticulum resident protein $44 \mathrm{OS}=$ Homo sapiens $\mathrm{GN}=\mathrm{ERP} 44 \mathrm{PE}=1 \mathrm{SV}=1$ \\
\hline 119,9 & 27 & T-complex protein 1 subunit delta $\mathrm{OS}=$ Homo sapiens $\mathrm{GN}=\mathrm{CCT} 4 \mathrm{PE}=2 \mathrm{SV}=1$ \\
\hline 104,4 & 12 & Suppressor of G2 allele of SKP1 homolog OS=Homo sapiens GN=SUGT1 PE=1 SV=3 \\
\hline 97,59 & 3 & Fanconi anemia group I protein $\mathrm{OS}=$ Homo sapiens $\mathrm{GN}=\mathrm{FANCI} \mathrm{PE}=1 \mathrm{SV}=4$ \\
\hline 95,98 & 3 & Nuclear pore complex protein Nup93 OS=Homo sapiens GN=NUP93 PE=1 SV=2 \\
\hline 95,84 & 58 & $\mathrm{CAD}$ protein $\mathrm{OS}=$ Homo sapiens $\mathrm{GN}=\mathrm{CAD} \mathrm{PE}=2 \mathrm{SV}=1$ \\
\hline 94,97 & 21 & T-complex protein 1 subunit eta $\mathrm{OS}=$ Homo sapiens $\mathrm{GN}=\mathrm{CCT} 7 \mathrm{PE}=1 \mathrm{SV}=2$ \\
\hline
\end{tabular}




\begin{tabular}{|c|c|c|}
\hline $\mathrm{H} / \mathrm{L}$ & \# & Description \\
\hline 88,83 & 8 & Exportin-1 OS=Homo sapiens GN=XPO1 PE $=1 \mathrm{SV}=1$ \\
\hline 83,98 & 5 & Fatty acyl-CoA reductase $1 \mathrm{OS}=$ Homo sapiens $\mathrm{GN}=\mathrm{FAR} 1 \mathrm{PE}=1 \mathrm{SV}=1$ \\
\hline 81,96 & 34 & T-complex protein 1 subunit epsilon $\mathrm{OS}=$ Homo sapiens $\mathrm{GN}=\mathrm{CCT} 5 \mathrm{PE}=2 \mathrm{SV}=1$ \\
\hline 80,57 & 2 & Serine palmitoyltransferase $1 \mathrm{OS}=$ Homo sapiens $\mathrm{GN}=\mathrm{SPTLC} 1 \mathrm{PE}=1 \mathrm{SV}=1$ \\
\hline 75,96 & 34 & T-complex protein 1 subunit beta OS=Homo sapiens $\mathrm{GN}=\mathrm{CCT} 2 \mathrm{PE}=1 \mathrm{SV}=4$ \\
\hline 72,18 & 3 & HCLS1-associated protein X-1 OS=Homo sapiens GN=HAX1 PE=1 SV=2 \\
\hline 71,11 & 20 & Insulin receptor substrate $4 \mathrm{OS}=$ Homo sapiens $\mathrm{GN}=\mathrm{IRS} 4 \mathrm{PE}=1 \mathrm{SV}=1$ \\
\hline 70,75 & 22 & Apoptosis-inducing factor 1, mitochondrial OS=Homo sapiens GN=AIFM1 PE=1 SV=1 \\
\hline 67,69 & 4 & Importin-4 OS=Homo sapiens GN=IPO4 PE=1 SV=2 \\
\hline 66,26 & 15 & Translational activator GCN1 OS=Homo sapiens GN=GCN1L1 PE $=1 \mathrm{SV}=6$ \\
\hline 62,46 & 2 & Tubulin beta- 3 chain $\mathrm{OS}=$ Homo sapiens $\mathrm{GN}=\mathrm{TUBB} 3 \mathrm{PE}=1 \mathrm{SV}=2$ \\
\hline 59,68 & 5 & Tubulin beta- 6 chain $\mathrm{OS}=$ Homo sapiens $\mathrm{GN}=\mathrm{TUBB} 6 \mathrm{PE}=1 \mathrm{SV}=1$ \\
\hline 56,91 & 15 & Protein transport protein $\mathrm{Sec} 16 \mathrm{~A} \mathrm{OS}=$ Homo sapiens $\mathrm{GN}=\mathrm{SEC} 16 \mathrm{~A} \mathrm{PE}=2 \mathrm{SV}=1$ \\
\hline 56,82 & 10 & Cullin-associated NEDD8-dissociated protein $2 \mathrm{OS}=$ Homo sapiens GN=CAND2 PE=1 SV=3 \\
\hline 56,48 & 2 & Cell division control protein 45 homolog OS=Homo sapiens GN=CDC45 PE=1 SV=1 \\
\hline 55,63 & 3 & Prostaglandin E synthase $3 \mathrm{OS}=$ Homo sapiens GN=PTGES3 PE=2 SV=1 \\
\hline 55,2 & 3 & Heat shock $70 \mathrm{kDa}$ protein $4 \mathrm{~L} \mathrm{OS}=$ Homo sapiens $\mathrm{GN}=\mathrm{HSPA} 4 \mathrm{~L} \mathrm{PE}=2 \mathrm{SV}=2$ \\
\hline 53,16 & 4 & E3 ubiquitin-protein ligase HUWE1 (Fragment) $\mathrm{OS}=$ Homo sapiens GN=HUWE1 PE=4 SV=1 \\
\hline 51,11 & 11 & DnaJ homolog subfamily A member 2 OS=Homo sapiens GN=DNAJA2 PE=1 SV=1 \\
\hline 49,17 & 4 & Neutral alpha-glucosidase $\mathrm{AB}$ OS=Homo sapiens GN=GANAB PE=2 SV=1 \\
\hline 49,05 & 82 & DNA-dependent protein kinase catalytic subunit $\mathrm{OS}=$ Homo sapiens $\mathrm{GN}=\mathrm{PRKDC} \mathrm{PE}=1 \mathrm{SV}=3$ \\
\hline 45,05 & 2 & RNA polymerase II-associated protein $3 \mathrm{OS}=$ Homo sapiens $\mathrm{GN}=\mathrm{RPAP} 3 \mathrm{PE}=1 \mathrm{SV}=2$ \\
\hline 42,32 & 34 & Heat shock protein HSP 90-alpha OS=Homo sapiens GN=HSP90AA1 PE=1 SV=5 \\
\hline 37,96 & 51 & Heat shock cognate $71 \mathrm{kDa}$ protein OS=Homo sapiens GN=HSPA8 PE $=1 \mathrm{SV}=1$ \\
\hline 37,68 & 16 & Tubulin beta chain OS=Homo sapiens $\mathrm{GN}=\mathrm{TUBB} \mathrm{PE}=1 \mathrm{SV}=2$ \\
\hline 37,06 & 7 & Mitochondrial import inner membrane translocase subunit TIM50 OS=Homo sapiens GN=TIMM50 PE=1 SV=2 \\
\hline 36,22 & 22 & Stress-70 protein, mitochondrial OS=Homo sapiens GN=HSPA9 PE=1 SV=2 \\
\hline 35,63 & 50 & Heat shock $70 \mathrm{kDa}$ protein $1 \mathrm{~A} / 1 \mathrm{~B} \mathrm{OS}=$ Homo sapiens $\mathrm{GN}=\mathrm{HSPA} 1 \mathrm{~A} \mathrm{PE}=1 \mathrm{SV}=5$ \\
\hline 35,47 & 3 & Ankyrin repeat domain-containing protein $13 \mathrm{~A} \mathrm{OS}=$ Homo sapiens $\mathrm{GN}=\mathrm{ANKRD} 13 \mathrm{~A} \mathrm{PE}=1 \mathrm{SV}=3$ \\
\hline 34,21 & 2 & Uncharacterized protein $\mathrm{OS}=\mathrm{Homo}$ sapiens $\mathrm{GN}=\mathrm{CSE} 1 \mathrm{~L} \mathrm{PE}=2 \mathrm{SV}=1$ \\
\hline 33,97 & 2 & Glutaryl-CoA dehydrogenase, mitochondrial OS=Homo sapiens GN=GCDH PE=1 SV=1 \\
\hline 33,66 & 2 & Serpin H1 OS=Homo sapiens GN=SERPINH1 PE=1 SV=2 \\
\hline 32,38 & 6 & ADP/ATP translocase $2 \mathrm{OS}=$ Homo sapiens $\mathrm{GN}=\mathrm{SLC} 25 \mathrm{~A} 5 \mathrm{PE}=1 \mathrm{SV}=7$ \\
\hline 32,28 & 2 & Phospholipase D3 (Fragment) OS=Homo sapiens GN=PLD3 PE=4 SV=1 \\
\hline 32,01 & 3 & BAG family molecular chaperone regulator $2 \mathrm{OS}=$ Homo sapiens $\mathrm{GN}=\mathrm{BAG} 2 \mathrm{PE}=1 \mathrm{SV}=1$ \\
\hline 31,38 & 6 & Stress-induced-phosphoprotein $1 \mathrm{OS}=$ Homo sapiens GN=STIP1 PE=4 SV=1 \\
\hline 30,39 & 3 & Ataxin-10 OS=Homo sapiens GN=ATXN10 PE=1 SV=1 \\
\hline 28,83 & 6 & Phosphate carrier protein, mitochondrial $\mathrm{OS}=\mathrm{Homo}$ sapiens $\mathrm{GN}=\mathrm{SLC} 25 \mathrm{~A} 3 \mathrm{PE}=1 \mathrm{SV}=2$ \\
\hline 28,33 & 7 & DNA damage-binding protein $1 \mathrm{OS}=$ Homo sapiens $\mathrm{GN}=\mathrm{DDB} 1 \mathrm{PE}=1 \mathrm{SV}=1$ \\
\hline 28 & 2 & DNA mismatch repair protein Msh6 OS=Homo sapiens GN=MSH6 PE $=2 \mathrm{SV}=1$ \\
\hline 27,41 & 10 & $60 \mathrm{kDa}$ heat shock protein, mitochondrial OS=Homo sapiens GN=HSPD1 PE=1 SV=2 \\
\hline 25,36 & 4 & Tubulin alpha-4A chain OS=Homo sapiens GN=TUBA4A PE=2 SV=1 \\
\hline 24,99 & 4 & RuvB-like $1 \mathrm{OS}=$ Homo sapiens $\mathrm{GN}=\mathrm{RUVBL} 1 \mathrm{PE}=1 \mathrm{SV}=1$ \\
\hline 24,5 & 3 & Tubulin beta- $4 \mathrm{~B}$ chain $\mathrm{OS}=$ Homo sapiens $\mathrm{GN}=\mathrm{TUBB} 4 \mathrm{~B} \mathrm{PE}=1 \mathrm{SV}=1$ \\
\hline 24,16 & 14 & RuvB-like 2 OS=Homo sapiens GN=RUVBL2 PE $=1 \mathrm{SV}=3$ \\
\hline 23,88 & 2 & Neutral amino acid transporter B(0) OS=Homo sapiens GN=SLC1A5 PE=2 SV=1 \\
\hline 23,41 & 11 & Galectin-3-binding protein OS=Homo sapiens GN=LGALS3BP PE=1 SV=1 \\
\hline 22,12 & 2 & Flotillin-2 OS=Homo sapiens GN=FLOT2 PE $=4 \mathrm{SV}=1$ \\
\hline 22,05 & 22 & $78 \mathrm{kDa}$ glucose-regulated protein $\mathrm{OS}=\mathrm{Homo}$ sapiens $\mathrm{GN}=\mathrm{HSPA} 5 \mathrm{PE}=1 \mathrm{SV}=2$ \\
\hline 21,79 & 29 & Heat shock protein HSP 90-beta OS=Homo sapiens GN=HSP90AB1 PE=1 SV=4 \\
\hline 21,35 & 2 & Importin-7 OS=Homo sapiens GN=IPO7 PE=1 SV=1 \\
\hline 21,13 & 2 & Sodium/potassium-transporting ATPase subunit alpha-1 OS=Homo sapiens GN=ATP1A1 PE=1 SV=1 \\
\hline 21,07 & 4 & Structural maintenance of chromosomes protein $2 \mathrm{OS}=$ Homo sapiens GN=SMC2 $\mathrm{PE}=1 \mathrm{SV}=2$ \\
\hline 20,94 & 4 & Heat shock $70 \mathrm{kDa}$ protein $6 \mathrm{OS}=$ Homo sapiens $\mathrm{GN}=\mathrm{HSPA} 6 \mathrm{PE}=1 \mathrm{SV}=2$ \\
\hline 20,69 & 7 & Pachytene checkpoint protein 2 homolog $\mathrm{OS}=$ Homo sapiens $\mathrm{GN}=\mathrm{TRIP} 13 \mathrm{PE}=1 \mathrm{SV}=2$ \\
\hline 20,45 & 5 & Reticulocalbin $-2 \mathrm{OS}=$ Homo sapiens $\mathrm{GN}=\mathrm{RCN} 2 \mathrm{PE}=2 \mathrm{SV}=1$ \\
\hline 20,44 & 3 & Tetratricopeptide repeat protein $27 \mathrm{OS}=$ Homo sapiens GN=TTC27 PE=1 SV=1 \\
\hline 20,44 & 3 & Emerin $\mathrm{OS}=$ Homo sapiens $\mathrm{GN}=\mathrm{EMD} \mathrm{PE}=4 \mathrm{SV}=1$ \\
\hline 19,83 & 24 & Tubulin alpha-1A chain OS=Homo sapiens GN=TUBA1A PE=1 SV=1 \\
\hline 19,71 & 2 & $\begin{array}{l}\text { Serine/threonine-protein phosphatase } 6 \text { regulatory ankyrin repeat subunit } \mathrm{C} \text { OS=Homo sapiens GN=ANKRD52 } \\
\mathrm{PE}=1 \mathrm{SV}=3\end{array}$ \\
\hline 19,05 & 4 & Gem-associated protein $4 \mathrm{OS}=$ Homo sapiens $\mathrm{GN}=\mathrm{GEMIN} 4 \mathrm{PE}=2 \mathrm{SV}=1$ \\
\hline 18,85 & 2 & Zinc transporter 7 (Fragment) OS=Homo sapiens GN=SLC30A7 PE=4 SV=2 \\
\hline 18,59 & 6 & Exportin-T OS=Homo sapiens GN=XPOT PE=1 SV=2 \\
\hline 18,49 & 2 & $\begin{array}{l}\text { Very-long-chain (3R)-3-hydroxyacyl-[acyl-carrier protein] dehydratase } 3 \text { OS=Homo sapiens GN=PTPLAD1 PE=2 } \\
\text { SV=1 }\end{array}$ \\
\hline 17,89 & 2 & DnaJ homolog subfamily B member 11 (Fragment) OS=Homo sapiens GN=DNAJB11 PE=4 SV=1 \\
\hline
\end{tabular}




\begin{tabular}{|c|c|c|}
\hline $\mathrm{H} / \mathrm{L}$ & \# & Description \\
\hline 17,15 & 4 & Sarcoplasmic/endoplasmic reticulum calcium ATPase $2 \mathrm{OS}=$ Homo sapiens GN=ATP2A2 PE $=1 \mathrm{SV}=1$ \\
\hline 16,45 & 3 & Protein arginine $\mathrm{N}$-methyltransferase $5 \mathrm{OS}=\mathrm{Homo}$ sapiens $\mathrm{GN}=\mathrm{PRMT} 5 \mathrm{PE}=1 \mathrm{SV}=4$ \\
\hline 15,97 & 6 & D-3-phosphoglycerate dehydrogenase OS=Homo sapiens GN=PHGDH PE=2 SV=1 \\
\hline 14,96 & 7 & Importin subunit beta-1 (Fragment) OS=Homo sapiens GN=KPNB1 PE=2 SV=1 \\
\hline 14,31 & 11 & Fatty acid synthase $\mathrm{OS}=\mathrm{Homo}$ sapiens $\mathrm{GN}=\mathrm{FASN} \mathrm{PE}=1 \mathrm{SV}=3$ \\
\hline 14,16 & 3 & $\begin{array}{l}\text { Complement component } 1 \text { Q subcomponent-binding protein, mitochondrial OS=Homo sapiens } \mathrm{GN}=\mathrm{C} 1 \mathrm{QBP} \mathrm{PE}=1 \\
\mathrm{SV}=1\end{array}$ \\
\hline 13,96 & 3 & Protein transport protein Sec61 subunit alpha isoform $1 \mathrm{OS}=$ Homo sapiens GN=SEC61A1 PE=2 SV=1 \\
\hline 13,77 & 2 & Serine/threonine-protein phosphatase 6 regulatory subunit $1 \mathrm{OS}=\mathrm{H}$ omo sapiens GN=PPP6R1 PE=1 SV=5 \\
\hline 13,61 & 2 & Nuclear migration protein nudC OS=Homo sapiens GN=NUDC $\mathrm{PE}=1 \mathrm{SV}=1$ \\
\hline 11,78 & 2 & Elongation factor 1-gamma $\mathrm{OS}=$ Homo sapiens $\mathrm{GN}=\mathrm{EEF} 1 \mathrm{G} \mathrm{PE}=2 \mathrm{SV}=1$ \\
\hline 11,58 & 2 & Keratin, type II cytoskeletal 8 OS=Homo sapiens GN=KRT8 PE=1 SV=7 \\
\hline 11,1 & 12 & Isoform 2 of Protein Hook homolog 2 OS=Homo sapiens GN=HOOK2 \\
\hline 10,83 & 3 & C-1-tetrahydrofolate synthase, cytoplasmic OS=Homo sapiens GN=MTHFD1 PE=1 SV=3 \\
\hline 10,48 & 3 & Uncharacterized protein $\mathrm{OS}=\mathrm{Homo}$ sapiens $\mathrm{GN}=\mathrm{P} 4 \mathrm{HB} \mathrm{PE}=3 \mathrm{SV}=1$ \\
\hline 9,522 & 5 & Heterogeneous nuclear ribonucleoprotein $\mathrm{F}$ OS=Homo sapiens $\mathrm{GN}=\mathrm{HNRNPF} \mathrm{PE}=1 \mathrm{SV}=3$ \\
\hline 9,077 & 6 & $26 \mathrm{~S}$ protease regulatory subunit $7 \mathrm{OS}=\mathrm{Homo}$ sapiens $\mathrm{GN}=\mathrm{PSMC} 2 \mathrm{PE}=1 \mathrm{SV}=3$ \\
\hline 8,891 & 6 & $26 \mathrm{~S}$ protease regulatory subunit $6 \mathrm{~B} \mathrm{OS}=\mathrm{Homo}$ sapiens $\mathrm{GN}=\mathrm{PSMC} 4 \mathrm{PE}=1 \mathrm{SV}=2$ \\
\hline 8,753 & 2 & 40S ribosomal protein $\mathrm{S} 14 \mathrm{OS}=$ Homo sapiens $\mathrm{GN}=\mathrm{RPS} 14 \mathrm{PE}=1 \mathrm{SV}=3$ \\
\hline 8,268 & 2 & Apolipoprotein D (Fragment) OS=Homo sapiens GN=APOD PE $=2 \mathrm{SV}=1$ \\
\hline 8,118 & 12 & Heat shock protein beta-1 OS=Homo sapiens GN=HSPB1 $\mathrm{PE}=1 \mathrm{SV}=2$ \\
\hline 7,941 & 5 & $\begin{array}{l}\text { Serine/threonine-protein phosphatase } 2 \mathrm{~A} 55 \mathrm{kDa} \text { regulatory subunit } \mathrm{B} \text { alpha isoform } \mathrm{OS}=\text { Homo sapiens } \\
\mathrm{GN}=\mathrm{PPP} 2 \mathrm{R} 2 \mathrm{~A} \mathrm{PE}=1 \mathrm{SV}=1\end{array}$ \\
\hline 7,926 & 25 & Probable ubiquitin carboxyl-terminal hydrolase FAF-X OS=Homo sapiens GN=USP9X PE=1 SV=3 \\
\hline 7,796 & 13 & Putative elongation factor 1-alpha-like $3 \mathrm{OS}=\mathrm{Homo}$ sapiens GN=EEF1A1P5 PE=5 SV=1 \\
\hline 7,763 & 8 & ATP synthase subunit alpha, mitochondrial OS=Homo sapiens GN=ATP5A1 PE=1 SV=1 \\
\hline 7,732 & 5 & Eukaryotic initiation factor 4A-I OS=Homo sapiens GN=EIF4A1 PE=1 SV=1 \\
\hline 7,424 & 2 & 26S proteasome non-ATPase regulatory subunit $1 \mathrm{OS}=$ Homo sapiens $\mathrm{GN}=\mathrm{PSMD} 1 \mathrm{PE}=1 \mathrm{SV}=2$ \\
\hline 7,175 & 2 & Eukaryotic translation initiation factor 3 subunit $\mathrm{E}$ OS=Homo sapiens GN=EIF3E PE=1 SV=1 \\
\hline 7,166 & 2 & $\begin{array}{l}\text { Serine/threonine-protein phosphatase } 2 \mathrm{~A} 65 \mathrm{kDa} \text { regulatory subunit A alpha isoform (Fragment) } \mathrm{OS}=\text { Homo sapiens } \\
\mathrm{GN}=\mathrm{PPP} 2 \mathrm{R} 1 \mathrm{~A} \mathrm{PE}=4 \mathrm{SV}=2\end{array}$ \\
\hline 7,04 & 5 & Peptidyl-prolyl cis-trans isomerase FKBP5 OS=Homo sapiens GN=FKBP5 PE=1 SV=2 \\
\hline 6,971 & 3 & Serine/threonine-protein phosphatase PGAM5, mitochondrial OS=Homo sapiens GN=PGAM5 PE=1 SV=2 \\
\hline 6,799 & 3 & Heterogeneous nuclear ribonucleoprotein $\mathrm{K}$ OS=Homo sapiens $\mathrm{GN}=\mathrm{HNRNPK} \mathrm{PE}=4 \mathrm{SV}=1$ \\
\hline 6,786 & 3 & 14-3-3 protein gamma OS=Homo sapiens $\mathrm{GN}=\mathrm{YWHAG} \mathrm{PE}=1 \mathrm{SV}=2$ \\
\hline 5,974 & 2 & Myosin light polypeptide $6 \mathrm{OS}=\mathrm{Homo}$ sapiens $\mathrm{GN}=\mathrm{MYL} 6 \mathrm{PE}=4 \mathrm{SV}=1$ \\
\hline 5,961 & 3 & Acyl-coenzyme A synthetase ACSM2B, mitochondrial (Fragment) OS=Homo sapiens GN=ACSM2B PE=2 SV=1 \\
\hline 5,867 & 8 & Histone deacetylase $6 \mathrm{OS}=\mathrm{Homo}$ sapiens $\mathrm{GN}=\mathrm{HDAC} 6 \mathrm{PE}=2 \mathrm{SV}=1$ \\
\hline 5,607 & 8 & $26 \mathrm{~S}$ protease regulatory subunit $8 \mathrm{OS}=\mathrm{Homo}$ sapiens $\mathrm{GN}=\mathrm{PSMC} 5 \mathrm{PE}=1 \mathrm{SV}=1$ \\
\hline 5,443 & 2 & Arginine--tRNA ligase, cytoplasmic OS=Homo sapiens GN=RARS PE=1 SV=2 \\
\hline 5,428 & 8 & 14-3-3 protein epsilon $\mathrm{OS}=\mathrm{Homo}$ sapiens $\mathrm{GN}=\mathrm{YWHAE} \mathrm{PE}=1 \mathrm{SV}=1$ \\
\hline 4,835 & 4 & Protein SET OS=Homo sapiens GN=SET PE $=1 \mathrm{SV}=3$ \\
\hline 4,639 & 3 & Guanine nucleotide-binding protein subunit beta-2-like $1 \mathrm{OS}=$ Homo sapiens $\mathrm{GN}=\mathrm{GNB} 2 \mathrm{~L} 1 \mathrm{PE}=2 \mathrm{SV}=1$ \\
\hline 4,622 & 45 & Ubiquitin (Fragment) OS=Homo sapiens GN=UBB PE=2 SV=1 \\
\hline 4,538 & 4 & 14-3-3 protein zeta/delta $\mathrm{OS}=$ Homo sapiens $\mathrm{GN}=\mathrm{YWHAZ} \mathrm{PE}=1 \mathrm{SV}=1$ \\
\hline 4,317 & 3 & Nuclear pore complex protein Nup205 OS=Homo sapiens GN=NUP205 PE=1 SV=3 \\
\hline 4,115 & 6 & CAS1 domain-containing protein 1 (Fragment) $\mathrm{OS}=$ Homo sapiens $\mathrm{GN}=\mathrm{CASD} 1 \mathrm{PE}=2 \mathrm{SV}=1$ \\
\hline 4,107 & 2 & $26 \mathrm{~S}$ protease regulatory subunit $4 \mathrm{OS}=$ Homo sapiens $\mathrm{GN}=\mathrm{PSMC} 1 \mathrm{PE}=2 \mathrm{SV}=1$ \\
\hline 3,661 & 4 & Transitional endoplasmic reticulum ATPase $\mathrm{OS}=$ Homo sapiens $\mathrm{GN}=\mathrm{VCP} \mathrm{PE}=1 \mathrm{SV}=4$ \\
\hline 3,634 & 8 & Actin, cytoplasmic $1 \mathrm{OS}=$ Homo sapiens $\mathrm{GN}=\mathrm{ACTB} \mathrm{PE}=1 \mathrm{SV}=1$ \\
\hline 3,445 & 2 & U5 small nuclear ribonucleoprotein $200 \mathrm{kDa}$ helicase $\mathrm{OS}=$ Homo sapiens $\mathrm{GN}=\mathrm{SNRNP} 200 \mathrm{PE}=1 \mathrm{SV}=2$ \\
\hline 3,41 & 3 & Elongation factor $2 \mathrm{OS}=\mathrm{Homo}$ sapiens $\mathrm{GN}=\mathrm{EEF} 2 \mathrm{PE}=1 \mathrm{SV}=4$ \\
\hline 3,363 & 23 & Phosphatidylinositol 4-kinase alpha OS=Homo sapiens GN=PI4KA PE=2 SV=1 \\
\hline 3,305 & 17 & Uncharacterized protein $\mathrm{OS}=\mathrm{Homo}$ sapiens $\mathrm{GN}=\mathrm{HDAC} 10 \mathrm{PE}=4 \mathrm{SV}=1$ \\
\hline 3,125 & 2 & Peroxiredoxin $-2 \mathrm{OS}=$ Homo sapiens $\mathrm{GN}=\mathrm{PRDX} 2 \mathrm{PE}=1 \mathrm{SV}=5$ \\
\hline 3,087 & 2 & CTP synthase OS=Homo sapiens GN=CTPS1 PE $=2 \mathrm{SV}=1$ \\
\hline 2,997 & 2 & 40S ribosomal protein $\mathrm{S} 20 \mathrm{OS}=$ Homo sapiens $\mathrm{GN}=\mathrm{RPS} 20 \mathrm{PE}=2 \mathrm{SV}=1$ \\
\hline 2,981 & 3 & Keratin, type I cuticular Ha1 OS=Homo sapiens GN=KRT31 PE=2 SV=3 \\
\hline 2,737 & 23 & Protein VAC14 homolog OS=Homo sapiens GN=VAC14 PE=1 SV=1 \\
\hline 2,727 & 19 & Transmembrane and coiled-coil domains protein $3 \mathrm{OS}=$ Homo sapiens $\mathrm{GN}=\mathrm{TMCC} 3 \mathrm{PE}=2 \mathrm{SV}=3$ \\
\hline 2,556 & 3 & Probable cytosolic iron-sulfur protein assembly protein $\mathrm{CIAO} 1 \mathrm{OS}=\mathrm{Homo}$ sapiens $\mathrm{GN}=\mathrm{CIAO} 1 \mathrm{PE}=1 \mathrm{SV}=1$ \\
\hline 2,401 & 8 & Polyphosphoinositide phosphatase OS=Homo sapiens GN=FIG4 PE=1 SV=1 \\
\hline 2,377 & 13 & 1-phosphatidylinositol 3-phosphate 5-kinase OS=Homo sapiens GN=PIKFYVE PE=1 SV=3 \\
\hline 2,375 & 4 & ATP-binding cassette sub-family A member $8 \mathrm{OS}=$ Homo sapiens $\mathrm{GN}=\mathrm{ABCA} 8 \mathrm{PE}=1 \mathrm{SV}=3$ \\
\hline 2,265 & 2 & Peroxisomal trans-2-enoyl-CoA reductase $\mathrm{OS}=$ Homo sapiens $\mathrm{GN}=\mathrm{PECR} \mathrm{PE}=1 \mathrm{SV}=2$ \\
\hline 2,245 & 30 & E3 ubiquitin-protein ligase TRIM21 OS=Homo sapiens GN=TRIM21 PE=2 SV=1 \\
\hline 2,149 & 403 & Keratin, type II cytoskeletal 2 epidermal OS=Homo sapiens GN=KRT2 PE=1 SV=2 \\
\hline
\end{tabular}




\begin{tabular}{ccl}
$\mathbf{H} / \mathbf{L}$ & $\#$ & Description \\
\hline 2,103 & 2 & Semaphorin-6B OS=Homo sapiens GN=SEMA6B PE=1 SV=4 \\
\hline 2,028 & 2 & Transmembrane and coiled-coil domains protein 1 OS=Homo sapiens GN=TMCC1 PE=2 SV=1 \\
\hline 2,026 & 3 & Peroxiredoxin-1 OS=Homo sapiens GN=PRDX1 PE=1 SV=1 \\
\hline 2,022 & 4 & 40S ribosomal protein S3 OS=Homo sapiens GN=RPS3 PE=2 SV=1 \\
\hline 1,992 & 2 & $\begin{array}{c}\text { Capping protein (Actin filament) muscle Z-line, beta, isoform CRA_a OS=Homo sapiens GN=CAPZB PE=4 } \\
\text { SV=1 }\end{array}$ \\
\hline 1,963 & 4 & 40S ribosomal protein SA (Fragment) OS=Homo sapiens GN=RPSA PE=3 SV=1 \\
\hline 1,946 & 2 & Glyceraldehyde-3-phosphate dehydrogenase OS=Homo sapiens GN=GAPDH PE=1 SV=3 \\
\hline 1,885 & 2 & Tetratricopeptide repeat protein $7 \mathrm{~B}$ OS=Homo sapiens GN=TTC7B PE=1 SV=3 \\
\hline 1,883 & 33 & Protein unc-13 homolog B OS=Homo sapiens GN=UNC13B PE=2 SV=1 \\
\hline 1,835 & 25 & Keratin, type II cytoskeletal lb OS=Homo sapiens GN=KRT77 PE=2 SV=3 \\
\hline 1,814 & 3 & BolA-like protein 2 OS=Homo sapiens GN=BOLA2 PE=1 SV=1
\end{tabular}

\subsection{Appendix C: List of Figures and Tables}

\subsubsection{Tables}

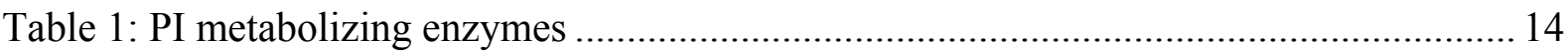

Table 2: Buffers and solutions used for molecular biology experiments ............................... 28

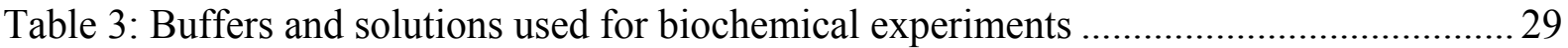

Table 4: Buffers and solutions used for cell biological experiments .................................... 30

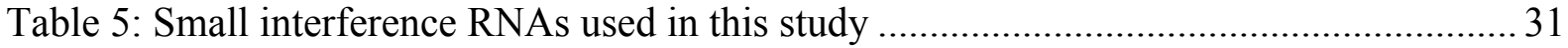

Table 6: Plasmid vector backbones used for cloning purposes and protein expression .......... 32

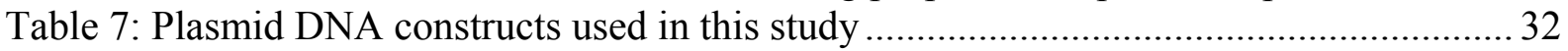

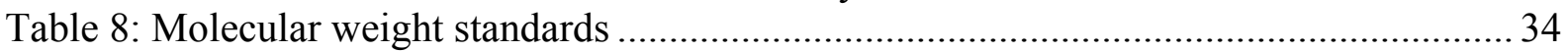

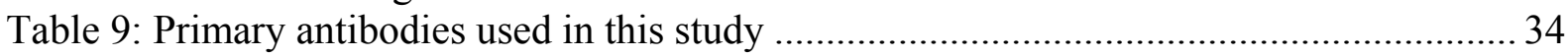

Table 10: MTM1 binding partner identified by MS/MS-based mass spectrometry .............. 133

Table 11: PI4K2 $\alpha$ binding partner identified by MS/MS-based mass spectrometry ............. 139

Table 12: DNA oligonucleotides used for sequencing and plasmid construction ................. 143

\subsubsection{Figures}

Figure 1: Distinct PI-pools as sign post of organelle identity in intracellular membrane traffic

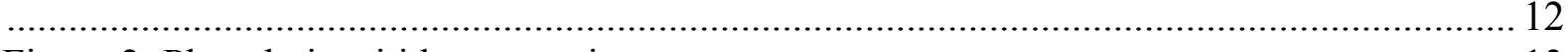

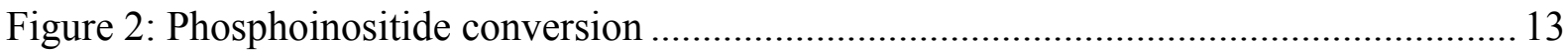

Figure 3: Tubular cargo-enriched subdomains at the early sorting endosome are formed by

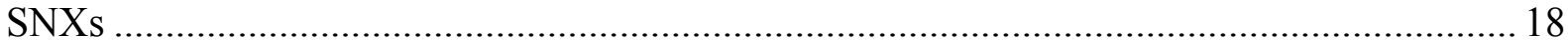

Figure 4: Endosomal trafficking is regulated by PI 3-kinases and phosphatases .................. 19

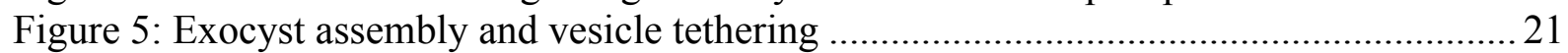

Figure 6: Domain structure and 'active/dead' combinations of the myotubularin family ........ 22

Figure 7: $\beta 1$-integrin accumulation in XLCNM patients can be rescued by re-expression of

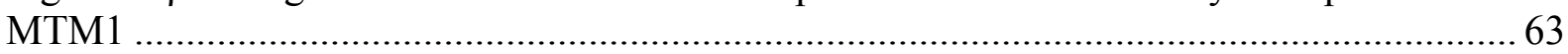

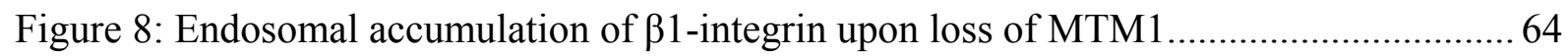

Figure 9: Defective Tf exocytosis in XLCNM patient cells ............................................... 65

Figure 10: Decreased TfR surface level as a readout for defective TfR recycling ...................67

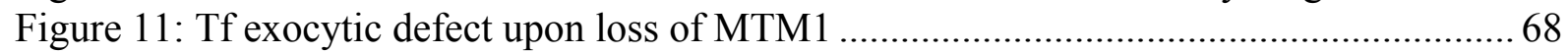

Figure 12: Accumulation of subplasmalemmal TfR-/ Tf-ligand containing endosomes in

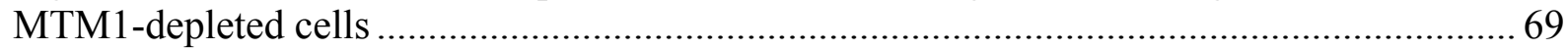

Figure 13: XLCNM-mutants of MTM1 are unable to rescue perinuclear loss of TfR in

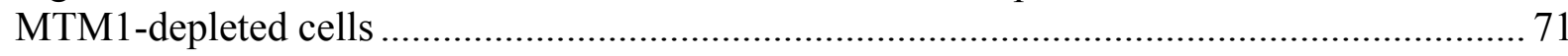


Figure 14: Endosomal trafficking in MTM1-depleted cells................................................ 72

Figure 15: Effects of depletion of other MTM family members........................................... 74

Figure 16: Endosomal compartments in MTM1-depleted cells .......................................... 76

Figure 17: Distinct early and recycling endosomal compartments in MTM1-depleted cells .. 77

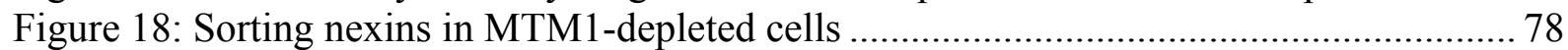

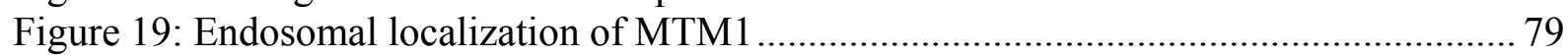

Figure 20: Semi-quantitative measurement of PI(3)P using recombinant PI(3)P-binding

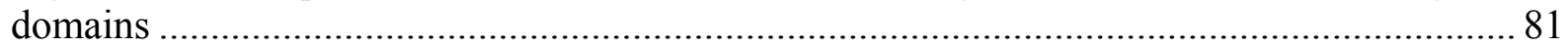

Figure 21: Selective increase in total PI(3)P levels and PI(3)P accumulation at TfR-cluster

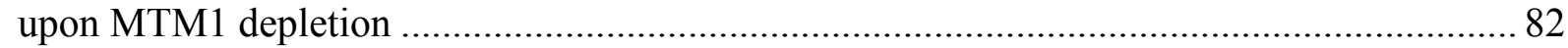

Figure 22: Removal of PI(3)P-binding effector proteins unmasked a previously not detectable

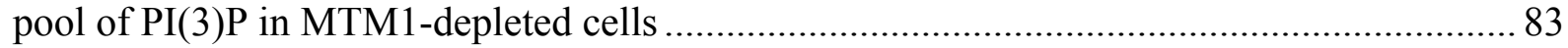

Figure 23: Reduction of PI(3)P synthesis counter-acts MTM1 loss-of-function defects ........ 84

Figure 24: Exogenous oversupply of PI(3)P phenocopies MTM1 depletion.......................... 84

Figure 25: Pharmacological inhibition of Vps34 rescues $\beta 1$-integrin accumulation in XLCNM

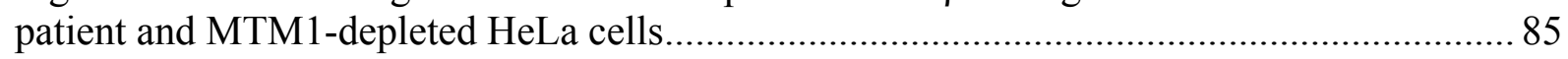

Figure 26: Manipulation of $\mathrm{PI}(3,5) \mathrm{P}_{2}$ - manipulation by PIKfyve depletion ......................... 86

Figure 27: PI(3)P-dependent outward trafficking of endosomes depends on Kif16b............. 88

Figure 28: Exocytic vesicle acquire PI(4)P prior to plasma membrane fusion....................... 89

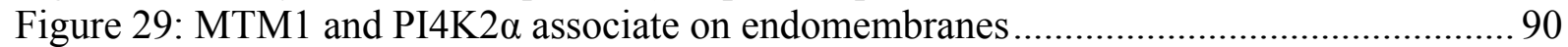

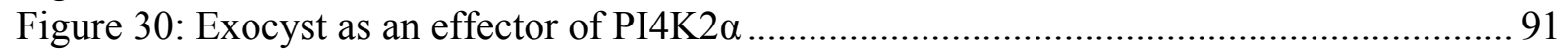

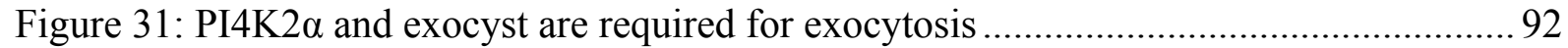

Figure 32: Rab11a-mediated exocyst recruitment to endosomes is required for exocytosis ... 93

Figure 33: Conversion of Rab5-to-Rab11-containing endosomes during exocytic recycling. 94

Figure 34: Membrane-recruitment of the exocyst complex depends on PI4K2 $\alpha$ and PI(4)P and

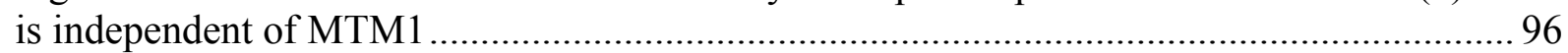

Figure 35: PI4K2 $\alpha$-dependent endosomal recruitment of MTM1 …................................... 97

Figure 36: PI4K2 $\alpha$-mediated recruitment of MTM1 initiates PI(3)P-to-PI(4)P conversion on

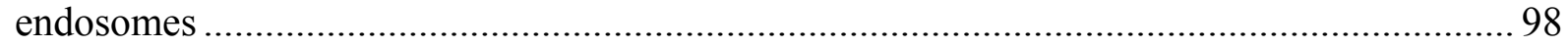

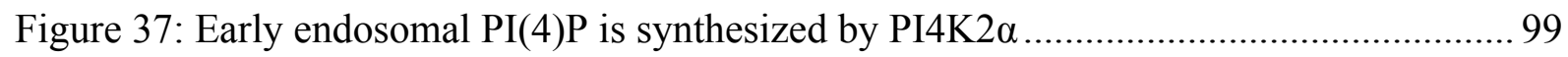

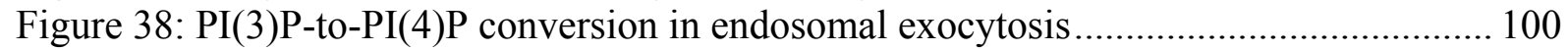

Figure 39: PI4K2 $\beta$ regulates endosomal exocytosis and exocyst function ............................ 107

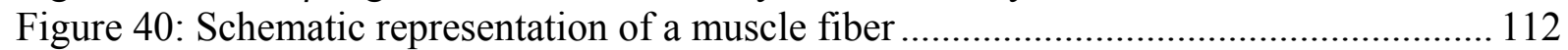

\subsection{Appendix D: Primers (DNA oligonucleotides)}

\section{Table 12: DNA oligonucleotides used for sequencing and plasmid construction}

primer are mostly named according to the following system: protein coding sequence - nucleotide position at which the primer starts - R or F (R: reverse; F: forward) - restriction enzyme binding site (if a restriction site is included in the primer an overhang of 4 randomly chosen bases, mostly ACTG was added at the 5'-end of the restriction site); seq - sequencing primer; wo stop - no stop codon within the 3' end of the reverse primer for Cterminally tagged proteins; tv - transcription variant; FL - primer used to amplify the full length construct; for reverse primers the reverse complement is listed

\begin{tabular}{lll} 
Primer name & Sequence 5' to 3' & $\begin{array}{l}\text { Targeted protein coding } \\
\text { sequence }\end{array}$ \\
\hline SNX1 1F EcoRI & Actggaattcatggcgtcgggtggtggtggc & human SNX1 tv1 \\
\hline SNX1 1560R NotI & cagtgeggecgcttaggagatggectttgcctcaggaagg & human SNX1 tv1; FL \\
\hline SNX3 1F EcoRI & Actggaattcatggcggagaccgtggctg & human SNX3 tv1 \\
\hline SNX3 586R NotI & cagtgcggccgctcaggcatgtcttattttagatggagtatag & human SNX3 tv1; FL \\
\hline SNX4 1F EcoRV & Actggatatcatggagcaggcacctccggaccc & human SNX4
\end{tabular}




\begin{tabular}{|c|c|c|}
\hline Primer name & Sequence 5' to 3' & $\begin{array}{l}\text { Targeted protein coding } \\
\text { sequence }\end{array}$ \\
\hline SNX4 1390R NotI & cagtgcggccgettacatcttgctaaagcattccttagcattggtcc & human SNX4; FL \\
\hline SNX8 1F EcoRV & Actggatatcatgactggccgcgcgatggacc & human SNX8 \\
\hline SNX8 1398R NotI & Cagtgcggccgcctagtgaggacacaggccgtcctc & human SNX8; FL \\
\hline SNX15 1F EcoRI & Actggaattcatgtcccgccaggcgaaggatgac & human SNX15 \\
\hline SNX17 1F EcoRI & actggaattcatgcacttttccattcccgaaaccgagtcc & human SNX17 \\
\hline SNX17 1413R NotI wo stop & cagtgcggccgcaacagatcctcatctccaatgecctcgaag & human SNX17; FL \\
\hline SNX27 1F EcoRV & Actggatatcatggcggacgaggacggggaag & human SNX27 \\
\hline SNX27 1623R NotI & cagtgcggecgectaatattcctcttttctccacttgagctcgc & human SNX27; FL \\
\hline SNX27 700F seq & Caattagatgcccgacgtcg & human SNX27 \\
\hline MTM1 366R seq & Tcctctacttgtcgcgcetc & human MTM1 \\
\hline MTM1 651F seq & ct tttaggtcccgaaatcga & human MTM1 \\
\hline MTM1 1003R seq & CAAACTGGACAACCAATGAG & human MTM1 \\
\hline MTM1 1F BamHI & actgggatccatggcttctgcatcaacttctaaatataattcacac & human MTM1 \\
\hline MTM1 R NotI & cagtgcggccgctcagaagtgagtttgcacatggggcat & human MTM1; FL \\
\hline MTM1 1F NheI & actggctagcatggettctgcatcaacttctaaatataattcacac & $\begin{array}{l}\text { human MTM1 } \\
\text { aa } 1-533\end{array}$ \\
\hline MTM1 1600R NotI & cagtgcggccgcttagagttccaagtgacgcatactggc & $\begin{array}{l}\text { human MTM1 } \\
\text { aa } 1-533\end{array}$ \\
\hline MTM1 mut oligo $\mathrm{R}$ & $\begin{array}{l}\text { CATTTACACTAGGCCTGGCATCATAAATGG } \\
\text { TGAGTTTAGAAATTTGTTTATTAGTCTCC }\end{array}$ & $\begin{array}{l}\text { human MTM1 } \\
\text { siRNA reistance }\end{array}$ \\
\hline MTM1 mut oligo F & $\begin{array}{l}\text { TATGATGCCAGGCCTAGTGTAAATGCAGTG } \\
\text { GCCAACAAG }\end{array}$ & $\begin{array}{l}\text { human MTM1 } \\
\text { siRNA reistance }\end{array}$ \\
\hline MTM1 C375S F & Cttgtgcattccagtgacggatgggacaggactg & human MTM1 \\
\hline MTM1 C375S R & Ccatccgtcactggaatgcacaagcactgaactc & human MTM1 \\
\hline MTM1 R241L F & Ggtcattgtgctttgcagtcagcctcttgtcggtatg & human MTM1 \\
\hline MTM1 R241L R & ggctgactgcaaagcacaatgaccgtcttattttctggatg & human MTM1 \\
\hline MTM1 P205L F & $\begin{array}{l}\text { CTTTTGGTGGTTCTGTATCGTGCCTCAGATG } \\
\text { ATGACCT }\end{array}$ & human MTM1 \\
\hline MTM1 P205L R & Gaggcacgatacagaaccaccaaaagagcaggg & human MTM1 \\
\hline MTM1 Y397C F & $\begin{array}{l}\text { GTTGGATAGCTTCTGTAGGAGCATTGAAGG } \\
\text { GTTCG }\end{array}$ & human MTM1 \\
\hline MTM1 Y397C R & $\begin{array}{l}\text { CAATGCTCCTACAGAAGCTATCCAACATCA } \\
\text { GCATGGC }\end{array}$ & human MTM1 \\
\hline MTMR1 608F seq & Cctcaacaagcatgcttttc & mouse MTMR1 \\
\hline MTMR1 1309F seq & Ccgtggtcatccactgcagtg & mouse MTMR1 \\
\hline MTMR1 1804F seq & Gtaaattattatgtacggtg & mouse MTMR1 \\
\hline MTMR1 -6F BamHI & Actgggatcccgcgtcatggacaggccagtgg & $\begin{array}{l}\text { mouse MTMR1, starts 6nt } \\
\text { before start codon }\end{array}$ \\
\hline MTMR1 R XhoI & cagtctcgagtcagactgaggtgtgtacaggagtagctg & mouse MTMR1; FL \\
\hline MTMR2 1F NotI & Actggcggecgcatggagaagagctcgagctgcga & human MTMR2 \\
\hline MTMR2 R XbaI & cagttctagattatacaacagtttggacaggagtgacac & human MTMR2; FL \\
\hline MTMR4 710F seq & Gcaatgctgatgatgagt & human MTMR4 \\
\hline MTMR4 1430F seq & Tcccetgcetgtttgaat & human MTMR4 \\
\hline MTMR4 2149F seq & Tcctagaagagactaagg & human MTMR4 \\
\hline MTMR4 2870F seq & Gttctagtcattccaatgg & human MTMR4 \\
\hline MTMR7 299F seq & caaggccagt gaaatatgag & human MTMR7 \\
\hline MTMR7 701F seq & Acttcgtttatgtcgttgac & human MTMR7 \\
\hline MTMR7 1401F seq & Ggccgactacctgaatcctc & human MTMR7 \\
\hline MTMR7 1F BamHI & Actgggatccatggagcacatccgcacgcccaag & human MTMR7 \\
\hline MTMR7 1980R NotI & cagtgcggecgctcaggcagtgagaaacacggcttcatc & human MTMR7; FL \\
\hline
\end{tabular}




\subsection{Appendix E: Publications}

Ketel K, Krauss M, Nicot AS, Puchkov D, Wieffer M, Müller R, Subramanian D, Schultz C, Laporte J, Haucke V. (2016) A phosphoinositide conversion mechanism for exit from endosomes. Nature 529, 408-12 [doi: 10.1038/nature16516]

Reubold TF, Faelber K, Plattner N, Posor Y, Ketel K, Curth U, Schlegel J, Anand R, Manstein DJ, Noé F, Haucke V, Daumke O, Eschenburg S. (2015) Crystal structure of the dynamin tetramer. Nature 525, 404-8 [doi: 10.1038/nature14880]

Wieffer M, Cibrián Uhalte E, Posor Y, Otten C, Branz K, Schütz I, Mössinger J, Schu P, Abdelilah-Seyfried S, Krauß M, Haucke V. (2013) PI4K2ß/ AP-1-based TGN-endosomal sorting regulates Wnt signaling. Curr Biol. 23, 2185-90 [doi: 10.1016/j.cub.2013.09.017] 